Universidade de São Paulo

Instituto de Física

\title{
Efeitos Termoelétricos em sistemas nanoscópicos
}

\author{
Alberto Torres Riera Junior \\ Orientador: Antônio José Roque da Silva
}

Tese de doutorado apresentada ao Instituto de Física para a obtenção do título de Doutor em Ciências.

\section{Banca examinadora:}

Prof. Dr. Antônio José Roque da Silva (IFUSP)

Prof ${ }^{\mathrm{a}}$. Dr ${ }^{\mathrm{a}}$. Helena Maria Petrilli (IFUSP)

Prof. Dr. Luis Gregório Godoy de Vasconcellos Dias da Silva (IFUSP)

Prof. Dr. Flavio Orlando Plentz Filho (UFMG)

Prof. Dr. Roberto Hiroki Miwa (UFU)

São Paulo 
FICHA CATALOGRÁFICA

Preparada pelo Serviço de Biblioteca e Informação do Instituto de Física da Universidade de São Paulo

Riera Junior, Alberto Torres

Efeitos termoelétricos em sistemas nanoscópicos. São Paulo, 2013.

Tese (Doutorado) - Universidade de São Paulo. Instituto de Física. Depto. de Física dos Materiais e Mecânica.

Orientador: Prof. Dr. Antônio José Roque da Silva

Área de Concentração: Física

Unitermos: 1. Física da matéria condensada 2. Física do estado sólido; 3. Física computacional; 4. Propriedades dos materiais; 5 . Materiais nanoestruturados 


\section{Agradecimentos}

A todos os que me apoiaram e deram suporte para que chegasse até aqui. Especialmente à minha família e amigos.

Ao meu orientador, Antônio José Roque da Silva, e ao Prof. Adalberto Fazzio, pelas discussões e idéias. E aos alunos do grupo SAMPA.

Agradeço ao CNPq, à CAPES e ao INCT-Nanomateriais de Carbono pelo suporte financeiro, e ao CENAPAD-SP pelo tempo computacional. 



\section{Resumo}

Efeitos termoelétricos descrevem o surgimento de campos elétricos em função de gradientes de temperatura e vice-versa.

Neste trabalho investigamos as propriedades termoelétricas de materiais de baixa dimensionalidade e nanoestruturas através de cálculos de primeiros princípios das propriedades de transporte destes sistemas, usando o código TRANSAMPA, que é baseado em funções de Green fora do equilíbrio e do código SIESTA, baseado em teoria do funcional da densidade.

Inicialmente estudamos nanofitas de grafeno e como estas são alteradas pela presença de impurezas substitucionais de Boro e Nitrogênio. Entre os principais resultados, mostramos que fitas na configuração ferromagnética apresentam efeito Seebeck dependente do spin, que pode ser ajustado por efeito de campo. A seguir, vemos que o coeficiente Seebeck $(S)$ em bicamadas de grafeno pode ser ajustado por potenciais de gate, de forma a escolher os portadores de carga, atingindo $S=$ $\pm 250 \mu \mathrm{V} / \mathrm{K}$. Também estudamos a dependência de $S$ com a temperatura $(T)$ e o tamanho do gate, calculamos a condutividade térmica por dinâmica molecular e a eficiência termoelétrica $(Z T)$. Na seqüencia, mostramos que grafeno dopado com Mn mostra caloritrônica de spin ajustável via gate e como a termocorrente varia $\operatorname{com} T$ e $\Delta T$.

Finalmente, calculamos as propriedades termoelétrica de uma junção molecular Au-BDT-Au e como elas variam em função do alongamento da junção. Também propomos um esquema geral para maximizar $Z T$ de junções moleculares em geral. 


\section{Abstract}

Thermoelectric effects describe how electric fields arise in response to temperature gradients and vice versa.

In this thesis we investigate the thermoelectric properties of low-dimensional materials and nanostructures theoretically. We perform ab initio calculations of the electronic transport properties using the TRANSAMPA code, based in nonequilibrium Green's functions, and the SIESTA code, based in density functional theory.

First, we study graphene nanoribbons and how their properties are altered by substitutional impurities. Among our main results for this system, we show that ribbons in the ferromagnetic configuration present spin-dependent Seebeck effect, which can be tuned by a field effect.

We show that the Seebeck coefficient $(S)$ of bilayer graphene is highly tunable by a gate potential, with ambipolar behavior, reaching $S= \pm 250 \mu \mathrm{V} / \mathrm{K}$. We also study how $\mathrm{S}$ varies with temperature $(T)$ and gate length. We calculate its thermal conductivity by molecular dynamics, and its thermoelectric efficiency $(Z T)$.

Then, we show that Mn doped graphene features a gate-tunable spin-dependent $S$, which is robust under changes in $T$ and $\Delta T$, rendering this material suitable for spin caloritronics.

Finally, we calculate how the thermoelectric properties of an Au-BDT-Au molecular junction vary with mechanical stretching, and propose a general recipe to improve $Z T$ in molecular junctions in general. 


\section{Sumário}

\begin{tabular}{lll}
\hline 1 & Introdução & 9
\end{tabular}

1.1 Grafeno $\ldots \ldots \ldots \ldots \ldots$

1.2 Nanofitas de Grafeno . . . . . . . . . . . . . . . . . . . . . . . . . . 15

1.3 Bicamadas de Grafeno . . . . . . . . . . . . . . . . . . . . . . . 17

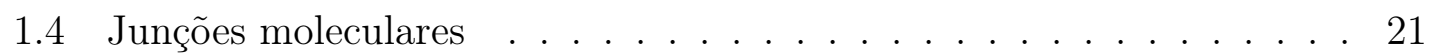

$\begin{array}{|ll|}2 & \text { Transporte de Carga e de Calor } \\ 23\end{array}$

2.1 Transporte de carga $\ldots \ldots \ldots \ldots$. . . . . . . . . . . . . 27

2.2 Transporte de calor . . . . . . . . . . . . . . . . . . . . . . . . . 34

2.3 Efeitos Termoelétricos . . . . . . . . . . . . . . . . . 36

$2.3 .1 \quad$ Efeito Seebeck . . . . . . . . . . . . . . . . . 36

2.3 .2 Efeito Peltier . . . . . . . . . . . . . . . . . 44

$2.3 .3 \quad$ Dispositivos termoelétricos . . . . . . . . . . . . . . . . . . . . 46

$2.3 .4 \quad$ Figura de Mérito adimensional $Z \theta$. . . . . . . . . . . . . 46

2.3 .5 Implementação computacional . . . . . . . . . . . . . . . 50

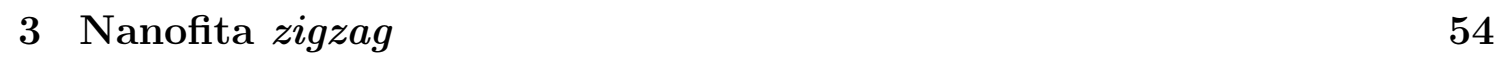

3.1 Efeito de campo na caloritrônica de spin . . . . . . . . . . . . . . . . 73

$\begin{array}{lll}4 & \text { Bicamada de Grafeno } & 80\end{array}$

\begin{tabular}{|lll}
5 & Caloritrônica de spin em Grafeno com Mn & 91
\end{tabular} 
6 Junção molecular Au-BDT-Au 103

$6.1 \quad$ Otimizando $Z T$ em junções moleculares. . . . . . . . . . . . . . . . . 112

\begin{tabular}{llr}
\hline 7 & Conclusões e Perspectivas & 120
\end{tabular}

7.1 Perspectivas . . . . . . . . . . . . . . . . . . . . . . 121

7.1.1 Propriedades termoelétricas de nanofitas com outros defeitos . 122

7.1.2 Transporte de calor da rede (fônons) . . . . . . . . . . . . . 123

7.1 .3 Desordem . . . . . . . . . . . . . . . . . . . 125

7.1 .4 Nanofios/Cadeias Au-Co . . . . . . . . . . . . . . . . . 127

A Teoria do Funcional da Densidade 130

A.1 Teoremas de Hohenberg-Kohn . . . . . . . . . . . . . . . . . . . 133

A.2 Ansatz de Kohn-Sham . . . . . . . . . . . . . . . . . . . . . . 134

A.3 Aproximação da Densidade Local (LDA) . . . . . . . . . . . . . . . 137

A.4 Aproximações Generalizadas de Gradiente (GGA) . . . . . . . . . . 138

\begin{tabular}{lll}
\hline B Aproximação de Born-Oppenheimer & 140
\end{tabular}

\begin{tabular}{ll}
\hline C Pseudopotenciais & 143
\end{tabular}

\begin{tabular}{ll}
\hline D Decimação & 145
\end{tabular}

E Acelerando cálculos de transporte usando CPUs e GPUs 155

\begin{tabular}{ll}
\hline Referências Bibliográficas & 165
\end{tabular} 


\section{Capítulo 1}

\section{Introdução}

Um dos maiores e mais importantes desafios que nossa sociedade enfrenta atualmente é adotar meios de desenvolvimento sustentáveis, que proporcionem crescimento econômico e, ao mesmo tempo, garantam que os recursos naturais do planeta não sejam exauridos em alguma data futura. Entre os maiores expoentes desse desafio estão os temas de meio ambiente e energia.

A ciência tem um papel principal na solução destes problemas, pois o desenvolvimento de novas tecnologias, métodos e processos que estejam de acordo com os preceitos da sustentabilidade, passa necessariamente por ela. Neste contexto, a busca de novos materiais que permitam o desenvolvimento de dispositivos que operem de forma mais eficiente, que sirvam como fontes de energia ou que possibilitem o reaproveitamento da mesma, adquire importância significativa, dada à sua capacidade de impacto sobre problemas de grande relevância para a sociedade.

Em particular, com o advento da nanotecnologia abriu-se um amplo leque de opções a ser explorado e que apresenta grande potencial para aplicações, que vão desde dispositivos eletrônicos (como transistores e diodos), sensores e células solares até spintrônica, entre outras. Entre alguns dos desenvolvimentos mais promissores neste campo está a descoberta de novos materiais de baixa dimensionalidade, como o grafeno [1] (2D) e as nanofitas de grafeno [2, 3] (1D), nanotubos de carbono [4] (1D), nanofios dos mais diversos materiais [5] (1D) e nanoestruturas como junções 
moleculares, por exemplo, e suas potenciais aplicações.

Efeitos termoelétricos [6, 7] relacionam diferenças de potencial com diferenças de temperatura entre as extremidades de um dado material. Assim, estes podem ser usados para gerar energia e recuperar desperdícios de calor, e como refrigeradores ou aquecedores [8, 9]. No entanto, devido à sua baixa eficiência, a aplicabilidade de dispositivos termoelétricos tem sido limitada [8, 10, 9, 11, 12]. De forma geral, estes dispositivos são usados em aplicações nas quais confiança e robustez são mais importantes do que a eficiência, como satélites e sondas espaciais (nas Voyager 1/2 e a Mars Cuiriosity rover, por exemplo) e em dutos de óleo e gás (para energizar sistemas de monitoramento e controle). Outras ocasiões nas quais dispositivos termoelétricos são preferidos são em situações onde portabilidade ou tamanho ou forma são fatores limitantes. Por exemplo, no resfriamento de pequenas regiões em circuitos integrados, em mini-refrigeradores de uso doméstico (para garrafas ou latas de bebidas) ou no controle térmico dos bancos em alguns modelos de carro.

Apesar de serem conhecidos há quase dois séculos (o efeito Seebeck foi descoberto em 1823), não se sabe muito sobre as propriedades termoelétricas desses novos materiais. Uma das razões óbvias para isso é que eles não existiam até pouco tempo atrás. Outra, é que apenas recentemente dispõe-se de meios (capacidade computacional) para realizar os cálculos necessários para o estudo das propriedades termoelétricas destes novos materiais [10] e, inclusive, de técnicas experimentais para medir efeitos termoelétricos em alguns tipos de nanoestruturas, como, por exemplo, junções moleculares, nas quais a primeira medida foi efetuada apenas em 2007 [13].

Nesta tese, estudamos as propriedades termoelétricas de sistemas nanoscópicos e materiais de baixa dimensionalidade, como grafeno e bicamadas de grafeno, nanofitas de grafeno e junções moleculares. Para tal fim, foram implementados diversos métodos para o cálculo destas propriedades no código de transporte TRANSAMPA [14, 15.

O texto está organizado da seguinte forma: Nas seções a seguir apresentamos 
os materiais e sistemas estudados nesta tese. No capítulo 2 descrevemos a teoria de transporte empregada nos cálculos realizados, os efeitos termoelétricos e a implementação computacional destas. Nos capítulos 3, 4, 5 e 6 apresentamos os resultados obtidos no estudo das propriedades termoelétricas de nanofitas de grafeno, bicamadas de grafeno, grafeno dopado com manganês e uma junção molecular, respectivamente. Na sequência, finalmente, apresentamos as conclusões e perspectivas de trabalho.

\subsection{Grafeno}

Desde a descoberta dos fulerenos por Kroto em 1989 [16] e dos nanotubos de carbono por Iijima em 1991 [17], materiais grafíticos de baixa dimensionalidade vêm atraindo cada vez mais interesse devido às excelentes propriedades mecânicas e eletrônicas que estes, de forma geral, possuem. O grafeno, apesar de já ser estudado teoricamente há 60 anos (grafite 2D) [18] para entender as propriedades de outros materiais grafíticos, somente foi isolado em laboratório recentemente, em 2004, por Novoselov et al[ [19].

Desde então, o grafeno tem sido alvo de intensa pesquisa, tornado-se um dos candidatos à substituição da tecnologia do silício e sendo, hoje em dia, um dos tópicos mais "quentes" na área de matéria condensada. Entre as várias propriedades que tornam o grafeno tão interessante, pode-se ressaltar a alta mobilidade dos portadores de carga (a maior já medida) [20], a maior resistência mecânica entre os materiais [21] e uma condutividade térmica duas vezes maior que a do diamante [22].

O grafeno, uma forma alotrópica do carbono constituída por uma camada de átomos dispostos numa estrutura hexagonal, pode ser considerado, do ponto de vista conceitual, como o bloco de construção de todos os outros materiais grafíticos

\footnotetext{
${ }^{1}$ Em 2010, Andre Geim e Konstantin Novoselov foram agraciados com o prêmio Nobel por seu trabalho com grafeno.
} 


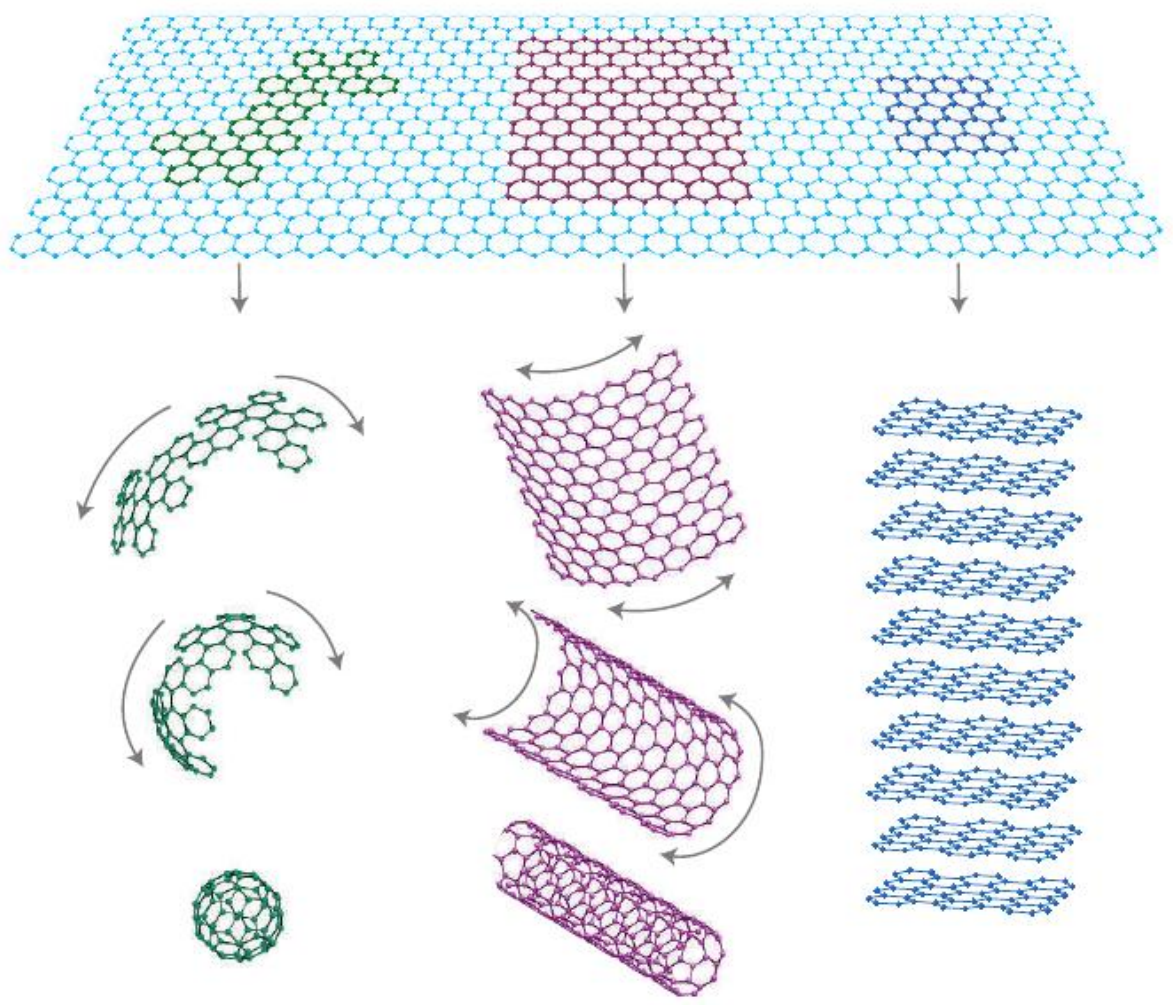

Figura 1.1: Pictoricamente, o grafeno pode ser embrulhado para formar fulerenos 0D, enrolado em nanotubos 1D ou empilhado, resultando em grafite 3D. Figura retirada da Ref. [1].

[1. Pictoricamente, ele pode ser embrulhado para formar fulerenos 0D, enrolado em nanotubos 1D ou empilhado, resultando em grafite 3D (Fig. 1.1).

A estrutura hexagonal do grafeno não é uma rede de Bravais, mas pode ser vista como uma rede triangular com dois átomos na base (formando duas sub-redes, A e B), conforme ilustrado na figura 1.2 . Os vetores de rede são dados por

$$
\begin{aligned}
& \mathbf{a}_{1}=\sqrt{3} a_{C C}\left(\frac{\sqrt{3}}{2}, \frac{1}{2}\right) \\
& \mathbf{a}_{2}=\sqrt{3} a_{C C}\left(\frac{\sqrt{3}}{2},-\frac{1}{2}\right)
\end{aligned}
$$

onde $a_{C C}=1.42 \AA$ é a distância carbono-carbono experimental e $\left|\mathbf{a}_{1}\right|=\left|\mathbf{a}_{2}\right|=$ $\sqrt{3} a_{C C}=2.46 \AA$ são os parâmetros de rede. Sua zona de Brillouin é então definida 
a)

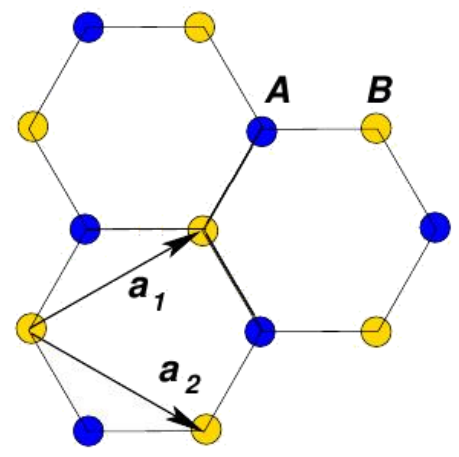

b)

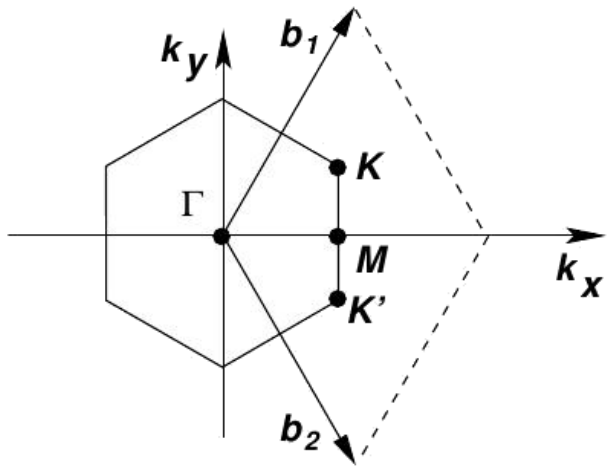

Figura 1.2: (a) Rede do grafeno, seus vetores de rede, $\mathbf{a}_{1}$ e $\mathbf{a}_{2}$ e as duas subredes, A (azul) e B (amarelo). (b) Primeira zona de Brillouin (hexágono), seus pontos de alta simetria e os vetores da rede recíproca, $\mathbf{b}_{1}$ e $\mathbf{b}_{2}$.

pelos vetores

$$
\begin{aligned}
& \mathbf{b}_{1}=\frac{2 \pi}{\sqrt{3} a_{C C}}\left(\frac{1}{\sqrt{3}}, 1\right) \\
& \mathbf{b}_{2}=\frac{2 \pi}{\sqrt{3} a_{C C}}\left(\frac{1}{\sqrt{3}},-1\right)
\end{aligned}
$$

onde $\left|\mathbf{b}_{1}\right|=\left|\mathbf{b}_{2}\right|=4 \pi / 3 a_{C C}$.

A estrutura de bandas do grafeno (Fig. 1.3) revela que este é um semicondutor de gap zero com um cruzamento linear no ponto K. Portanto, perto do ponto de condução, K, no chamado cone de Dirac, os elétrons apresentam uma massa efetiva nula, comportando-se como férmions de Dirac sem massa [1], o que dá origem a vários efeitos relativísticos como o efeito Hall quântico (que não serão tratados neste trabalho).

Os átomos de carbono (valência $2 s^{2} 2 p^{2}$ ) no grafeno apresentam hibridização $s p^{2}$, formando ligações $\sigma$ no plano. Já os orbitais $p_{z}$ não hibridizados, que apontam para fora do plano do grafeno, formam ligações $\pi$ deslocalizadas. As ligações $\sigma$ são ligações covalentes fortes, majoritariamente responsáveis pelas propriedades mecânicas e elásticas do grafeno, enquanto que as ligações $\pi$ são responsáveis pelas propriedades 


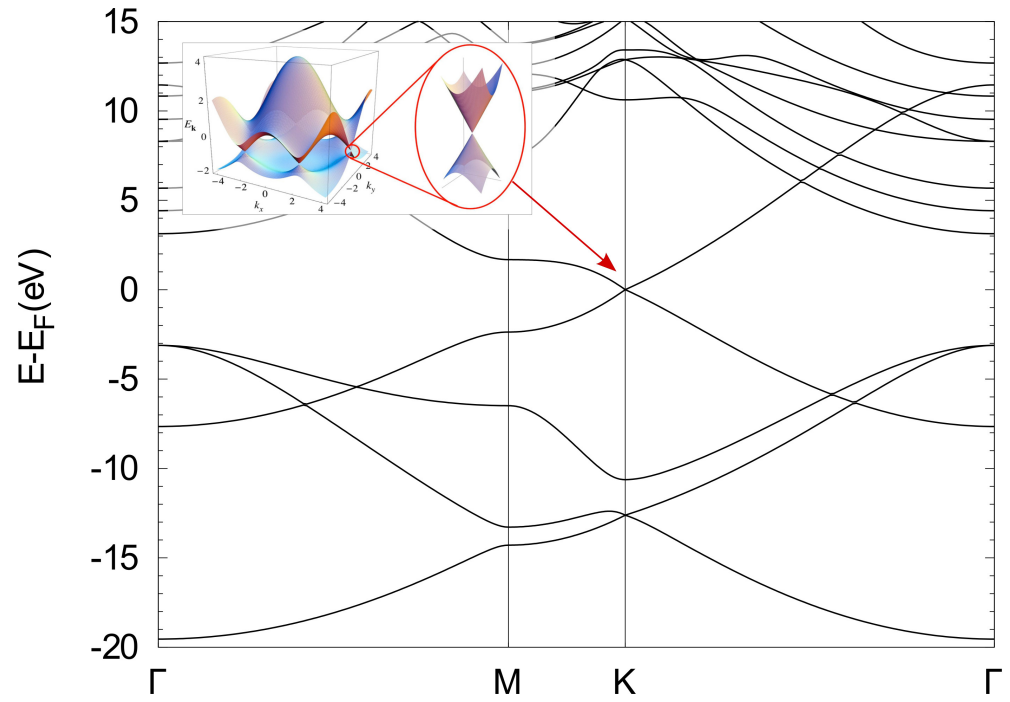

Figura 1.3: Estrutura de bandas do grafeno. Note o cruzamento linear no ponto K. No quadro menor, a estrutura de bandas $2 \mathrm{D}$ na proximidade do nível de Fermi, adaptado da Ref. [23].

eletrônicas [23].

As primeiras medidas de efeito termoelétrico em grafeno foram reportadas em 2009 por Y. M. Zuev et al. [24], P. Weng et al. [25] e J. G. Checkelsky et al. [26]. Nestes experimentos (veja a Fig. 1.4), os valores máximos obtidos para o coeficiente Seebeck, S, ou termopotência (TEP - Thermoelectric Power), que é a razão entre a voltagem termicamente induzida $\Delta V_{t h}$ e a diferença de temperatura aplicada $\Delta T$ (explicação detalhada na seção 2.3.1), à temperatura ambiente foram entre $50-$ $90 \mu \mathrm{V} / \mathrm{K}$. Para efeito de comparação, metais costumam ter coeficientes $S$ de alguns poucos $\mu \mathrm{V} / \mathrm{K}$ e semicondutores, de algumas centenas. Além disso, foi observada uma grande variação da TEP em função da voltagem de gate $V_{g}$, apresentando valores positivos para voltagens de gate negativas, nas quais os portadores de carga são buracos (veja os diagramas na Fig. 1.4 ) ; zero para voltagem nula, e negativa para voltagens de gate positivas, na qual a corrente é dada por elétrons. Vemos então que o coeficiente Seebeck é muito sensível à densidade e tipo de portadores de carga do grafeno, isto é, à dopagem. Inclusive, Sidorov et al. 27] recentemente demonstraram que grafeno pode ser usado como dispositivo para medir a concentração de gases (no 


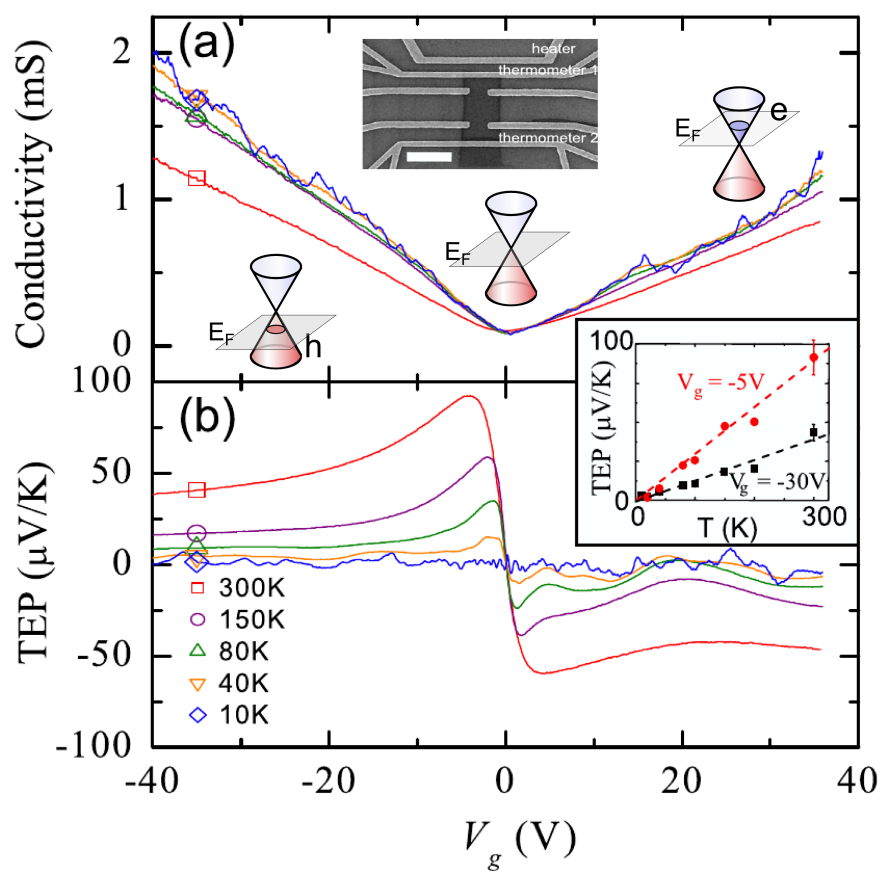

Figura 1.4: (a) Condutividade e (b) termopotência (TEP) de grafeno em função da voltagem de gate, $V_{g}$. O dispositivo usado para fazer a medida é mostrado no quadro em (a). No quadro em (b), TEP em função da temperatura. Figura adaptada da Ref. [24].

experimento foram usados ar, $\mathrm{N}_{2} \mathrm{O}$ e $\mathrm{NH}_{3}$ ).

\subsection{Nanofitas de Grafeno}

Nanofitas de grafeno (Graphene Nano-Ribbons - GNR) são formadas através do corte do grafeno. Dependendo da direção na qual o corte é feito são obtidas fitas com diferentes tipos de bordas. Na figura 1.5 podemos ver os dois tipos básicos de bordas. GNRs com bordas como as da Fig. 1.5a são chamadas de armchair e podem ser vistas como se fossem um nanotubo zigzag aberto. Se forem como as da Fig. 1.5b, são denominadas zigzag, estas podem ser vistas como se fossem um nanotubo armchair aberto. Misturas entre estes tipos de bordas também são possíveis.

O tipo de borda é uma característica importante, pois é ela que determina as propriedades eletrônicas das fitas. GNRs armchair, como seus nanotubos correspon- 
(a)

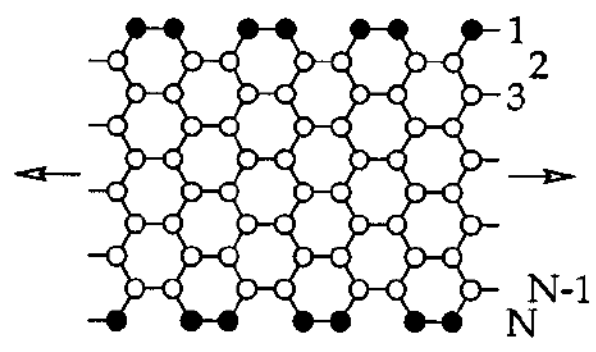

(b)

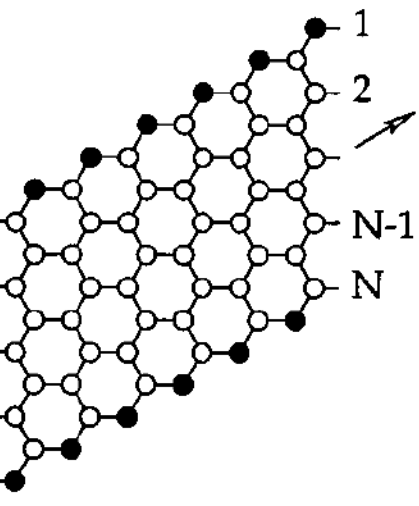

Figura 1.5: Exemplos da estrutura de nanofitas do tipo armchair (a) e zigzag (b). Figura retirada da Ref. [28].

dentes, são condutoras se $N=3 m-1$, onde $N$ é o número de linhas de átomos de Carbono (veja a Fig. 1.5) e $m$ é um número inteiro, e semicondutoras caso contrário [28, 3, 23]. Fitas zigzag, diferentemente do que acontece com nanotubos, não são condutoras sempre. Os estados mais estáveis de GNRs zigzag apresentam magnetismo e, dependendo do alinhamento dos momentos magnéticos das bordas, podem ser condutoras ou semicondutoras [29, 23, 30].

Além disso, fitas zigzag apresentam estados localizados nas bordas com energias próximas ao nível de Fermi. Na figura 1.6 podem-se ver duas imagens de STM, realizadas por Kobayashi et al. [31, 32, mostrando a presença de estados localizados em superfícies de grafeno com bordas hidrogenadas em grafite pirolítico altamente orientado (Highly Oriented Pyrolitic Graphite - HOPG), que aparecem como regiões mais brillantes nas imagens.

Devido às suas incríveis propriedades, o grafeno abriu um novo horizonte para possíveis aplicações. Entre os vários dispositivos que já foram demonstrados experimentalmente temos transistores de efeito de campo (Field Effect Transistors - FET) [33, 34], transistores de um único elétron (Single Electron Transistor - SET) 35, 36, 37], dispositivos de memória [38, 39], sensores ultra-sensíveis [40, 41, 42], células foto-voltáicas [43], materiais compostos [44], dispositivos spintrônicos [45, 46, 47, 48] 

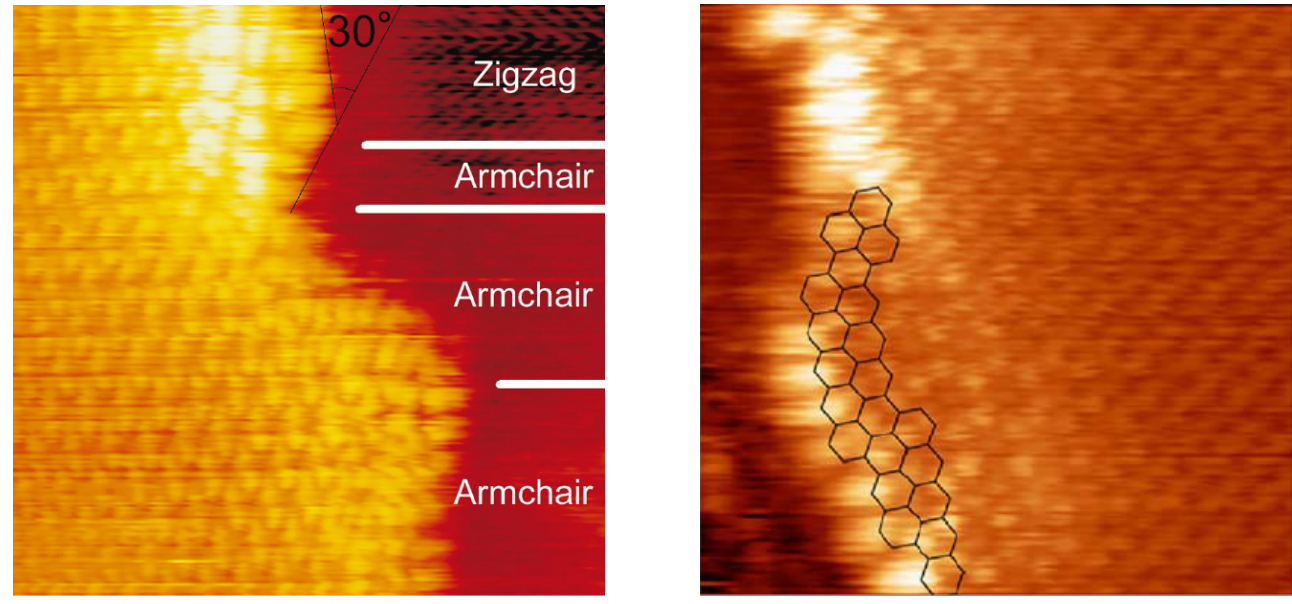

Figura 1.6: Imagens de STM em modo de corrente constante $\left(V_{s}=0.02 \mathrm{~V}\right.$ e $I=$ $0.7 n A)$ de superfícies de grafeno com bordas hidrogenadas em HPOG. As regiões mais claras indicam uma maior densidade local de estados. Figuras retiradas das refs. [31, 32].

e qubits de spin [49, 50].

A maioria dos dispositivos acima citados são baseados em nanofitas de grafeno. Portanto, é importante do ponto de vista de aplicações que seja possível produzir fitas estreitas e com bordas bem definidas (lembre que o tipo de borda define as propriedades eletrônicas da fita). Atualmente, estes objetivos já são alcançados em laboratório (embora não sempre ao mesmo tempo). Na figura 1.7, pode-se ver imagens AFM de nanofitas de até $20 \mathrm{~nm}$ com bordas bem definidas produzidas por meios químicos [2]. Já na figura 1.8 a tem-se uma fita armchair de aproximadamente $2.5 \mathrm{~nm}$ produzida por litografia STM (esta não apresenta bordas suaves).

\subsection{Bicamadas de Grafeno}

Bicamadas de grafeno são formadas pelo empilhamento de duas folhas de grafeno da mesma forma que no grafite, isto é, no empilhamento $\mathrm{AB}$, comumente chamado de Bernal. Neste tipo de empilhamento, metade dos átomos da camada superior está posicionada sobre átomos da camada inferior e a outra metade, sobre o centro dos hexágonos (veja a Fig. 1.9). 

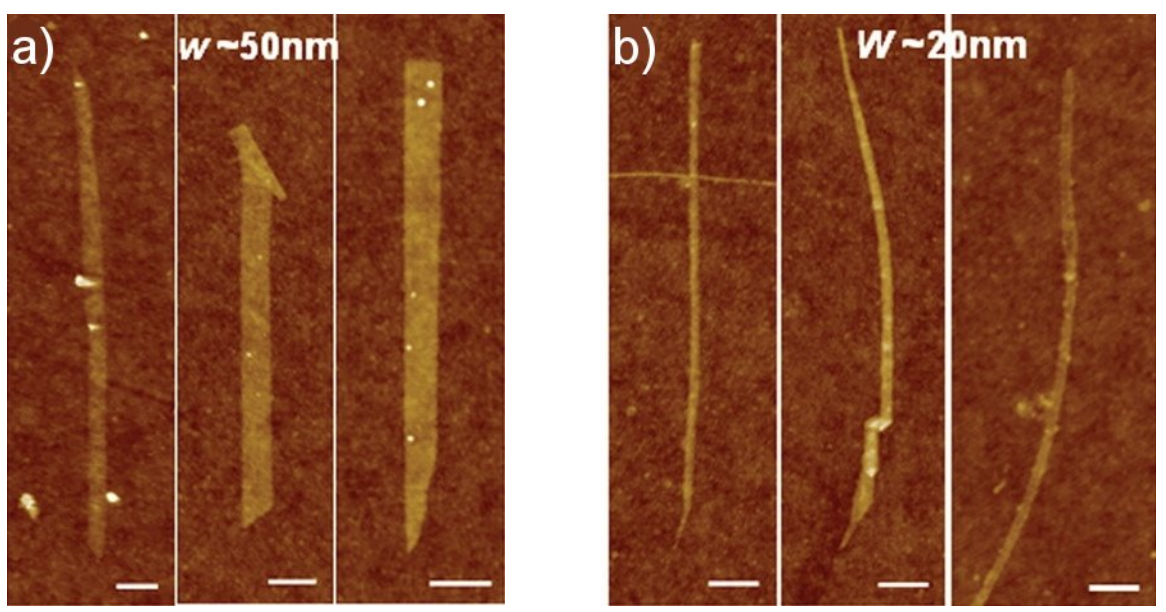

Figura 1.7: Imagens AFM de nanofitas de grafeno produzidas por meios químicos. A fitas em (a) possuem uma, duas e duas camadas respectivamente. Em (b), possuem de três a uma (direita). Todas as barras de escala indicam $100 \mathrm{~nm}$. Figuras retiradas da Ref. [2]
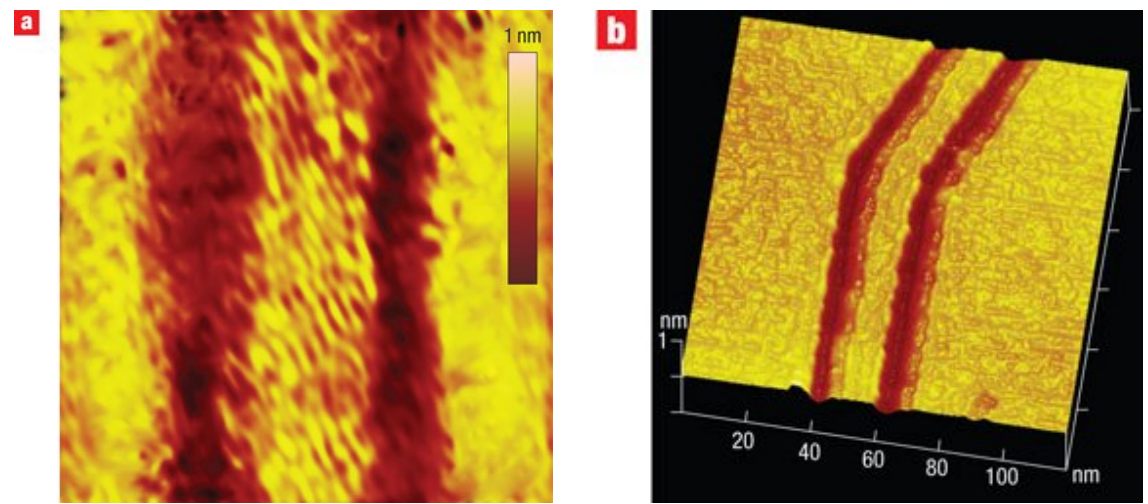

Figura 1.8: Imagens STM de (a) uma nanofita de grafeno armchair de $2.5 \mathrm{~nm}$ de largura e (b) de uma junção de uma fita armchair com uma zigzag, com largura de $8 \mathrm{~nm}$. Fitas produzidas por litografia STM. Figuras retiradas da Ref. [51]. 


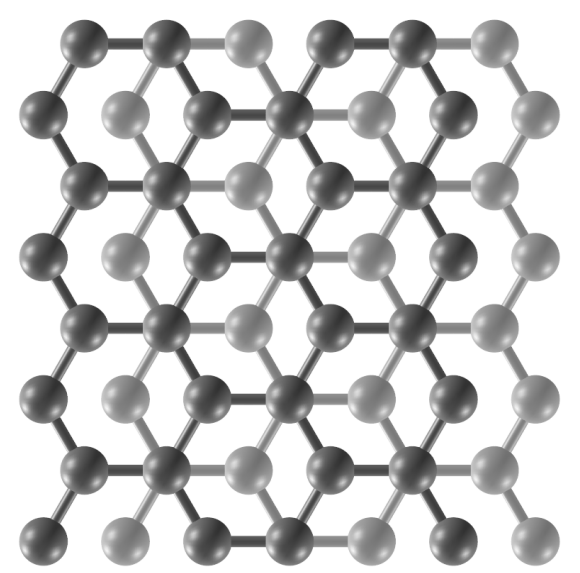

Figura 1.9: Visão esquemática de uma bicamada de grafeno no empilhamento de Bernal (AB). Metade do átomos estão localizados uns sobre os outros e a outra metade, centrada nos hexágonos.

Este material, assim como o grafeno, é um semicondutor de gap zero. No entanto, sua estrutura de bandas apresenta dispersão parabólica no ponto K (linha tracejada na Fig. 1.10k) [23]. Quando um campo elétrico é aplicado na bicamada, a simetria entre as duas camadas é quebrada pela transferência de carga resultante e se forma um gap de energia, $E_{g}$, na estrutura de bandas (linhas azuis e vermelhas na Fig. 1.10 c). Este gap de energia é altamente ajustável, tendo sido demonstrado experimentalmente que este pode ser variado de 0 até $\sim 300 \mathrm{meV}$ [52]. Na parte superior da figura 1.10b pode-se ver a grande variação na resistência, $R$, de uma bicamada de grafeno em função das voltagens de top/back gate, $V_{t g} / V_{b g}$ (Ref. [53]), o que é conseqüencia da variação do gap. Esta capacidade de ajustar $E_{g}$ pode ser muito importante para ajustar, também, o efeito termoelétrico, que costuma ser maior quando o material apresenta um gap na sua estrutura de bandas.

Os primeiros experimentos medindo o coeficiente Seebeck, $S$, em bicamadas de grafeno foram realizados em 2010 e 2011 por S-G Nam et al. [54] e C-R Wang et al. [53]. Nestes experimentos foram reportados valores de $S \sim 130 \mu \mathrm{V} / \mathrm{K}$ a $300 \mathrm{~K}$ [53], e de $S \sim 100 \mu \mathrm{V} / \mathrm{K}$ a $250 \mathrm{~K}$ [54]. Também foi verificado uma alto ajuste do coeficiente Seebeck (parte inferior da Fig. 1.10b) em função das voltagens de gate. 

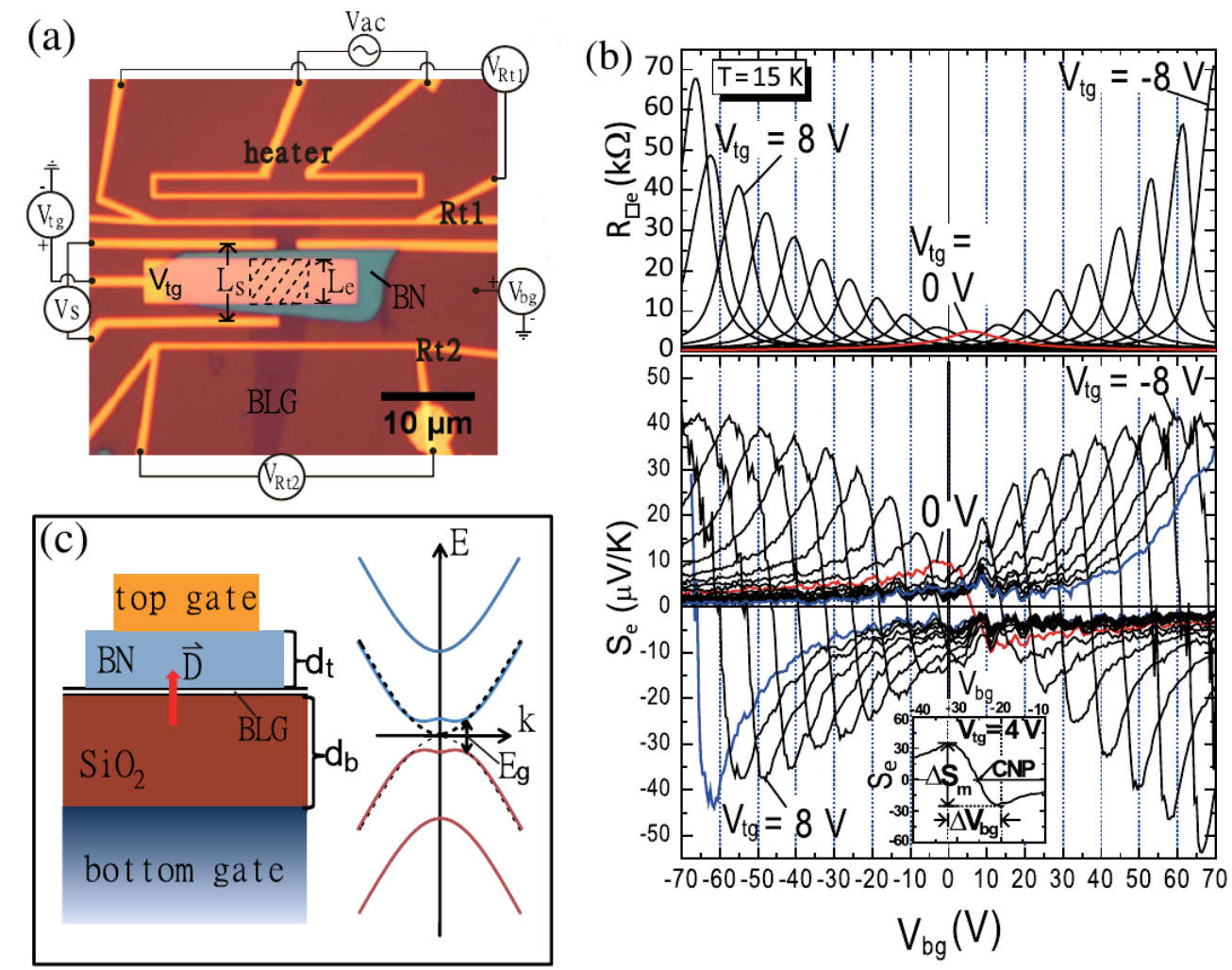

Figura 1.10: (a) Imagem do dispositivo de bicamada de grafeno usado para medir o coeficiente Seebeck. (b) Resistência (acima) e coeficiente Seebeck (abaixo) efetiva da região sob efeito do top gate em função das voltagens de top e back gate, $V_{t g}$ e $V_{b g}$ respectivamente. (c) Visão lateral esquemática do dispositivo (esquerda) e estrutura de bandas com e sem campo aplicado (linhas contínuas e tracejadas, respectivamente) (direita). Figuras retiradas da Ref. [53] 


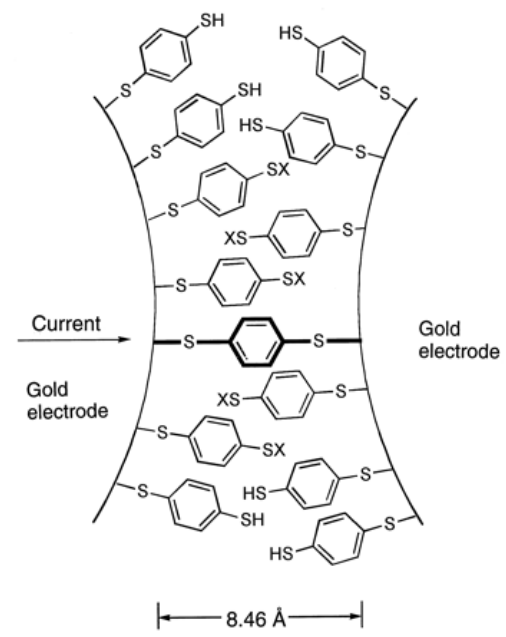

Figura 1.11: Visão esquemática da junção formada entre os contatos de ouro e a molécula de benzeno-1,4-ditiol (BDT). Fig. retirada da Ref. [56]

\subsection{Junções moleculares}

O campo da eletrônica molecular visa utilização de moléculas como componentes elementares na construção de circuitos [55]. Embora este objetivo ainda permaneça distante, o estudo das propriedades de transporte de moléculas tem atraído um grande interesse, desenvolvendo-se rapidamente. A primeira medida de condutância de uma única molécula foi realizada pelo grupo de James Tour em 1997 [56]. Neste experimento pioneiro, junções moleculares foram formadas através da técnica de quebra de junção mecanicamente controlável (MCBJ - Mechanically Controllable Break Junction), na qual um fio de ouro, sobre o qual se depositou uma camada de moléculas de benzeno-1,4-ditiol (BDT), foi sendo esticado até romper, formando duas pontas (os contatos) com a molécula de BDT "ensanduichada" no meio. A partir deste trabalho, a molécula de BDT se tornou a referência para comparação de resultados na área [57].

Dez anos mais tarde, em 2007, foi efetuada a primeira medida das propriedades termoelétricas de uma junção molecular de BDT. Neste experimento, A. Majumdar et al. [13] usaram a técnica de MCBJ controlada por um microscópio de tunelamento (STM-MCBJ) para formar e medir o coeficiente Seebeck em uma junção molecular 

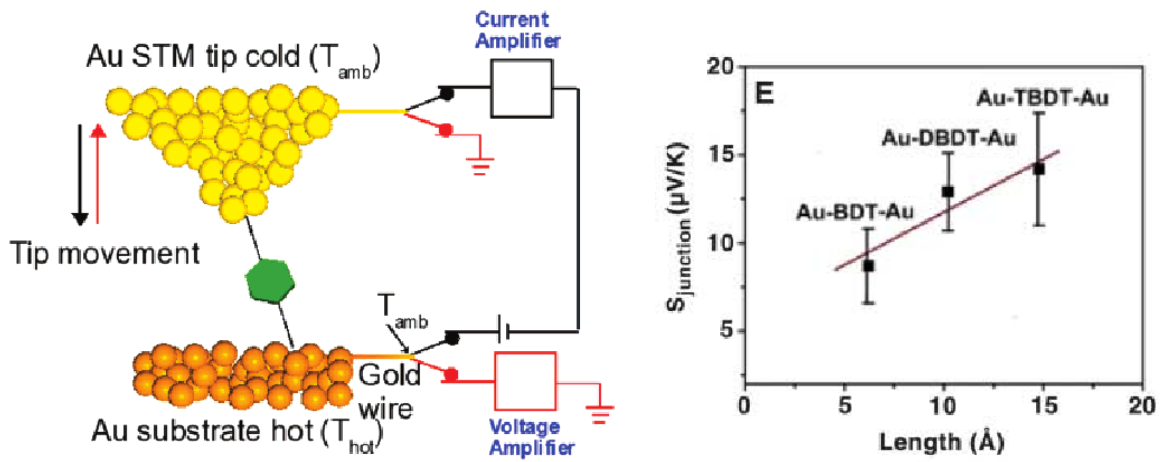

Figura 1.12: Visão esquemática do processo de medição do efeito Seebeck em uma junção molecular (esquerda). Coeficiente Seebeck para moléculas com 1,2 e 3 BDTs em cadeia (direita). Figs. retiradas da Ref. [13].

Au-BDT-Au. Basicamente, no experimento, uma monocamada de moléculas de BDT foi depositada sobre uma superfície de ouro e a ponta do STM foi aproximada e afastada repetidamente para formar as junções (veja a Fig. 1.12). Quando a condutância da junção, depois de formada, atingia um determinado valor $(G=$ $\left.0.1 \mathrm{G}_{0}\right)$ o afastamento era pausado e se aquecia a superfície acima da temperatura ambiente. A partir das medidas da corrente resultante, foi determinado o coeficiente Seebeck. Apesar do valor medido para o BDT ter sido baixo, de $+8.7 \pm 2.1 \mu \mathrm{V} / \mathrm{K}$, foi possível determinar, pelo sinal de $S$, que os portadores de carga nesta junção são buracos. Ou seja, medidas de $S$ permitem determinar a natureza dos portadores de carga. 


\section{Capítulo 2}

\section{Transporte de Carga e de Calor}

Entender as propriedades de transporte de carga e de calor em sistemas nanoscópicos é uma tarefa fundamental, pois boa parte das aplicações potenciais em nanotecnologia se baseia em fenômenos de transporte. Um exemplo claro são os dispositivos eletrônicos. Contudo, dada a natureza deste tipo de sistemas, uma descrição totalmente quântica do problema se faz necessária, o que não é fácil, dada a complexidade dos mesmos.

Os sistemas que estamos interessados em estudar são dispositivos de duas pontas conectadas por um objeto nanoscópico. Assim, podemos dividir o sistema em três regiões, os eletrodos (as pontas) e a região central, que pode ser uma molécula ou uma nanoestrutura qualquer. O transporte neste tipo de sistemas pode ser visto sob três aspectos distintos (veja a figura 2.1): da termodinâmica, da quântica e da eletrostática.

Do ponto de vista da termodinâmica, temos dois reservatórios conectados pelo objeto nanoscópico. Neste caso, o fluxo (carga, calor) entre os reservatórios esquerdo e direito é governado pelas diferenças do potencial químico e da temperatura entre os mesmos, que ditam o estado estacionário do sistema.

Já do ponto de vista eletrostático, o perfil do potencial ao longo do dispositivo determina as forças (i.e., o campo) que atuam sobre os elétrons. Uma aproximação 

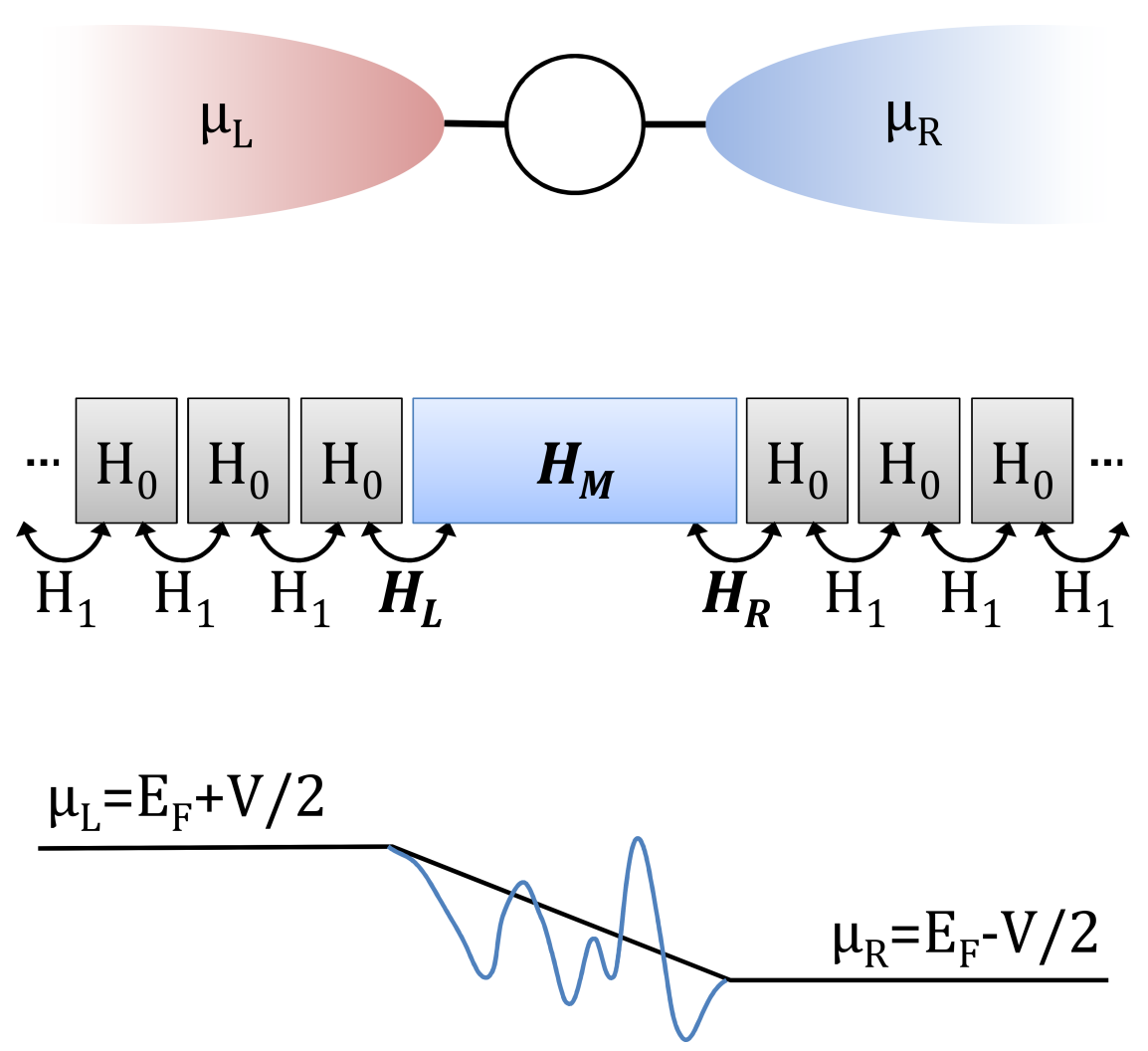

Figura 2.1: O problema de transporte entre dois contatos ligados por um objeto nanoscópico visto por três perspectivas diferentes: termodinâmica, mecânica quântica e eletrostática, respectivamente. 


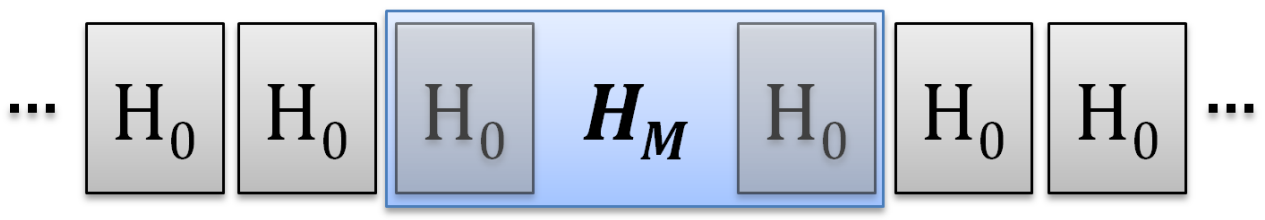

Figura 2.2: Para garantir que a queda de potencial aconteça na região central, esta deve conter "pedaços" dos eletrodos que blindem as variações de carga (potencial).

que fazemos aqui é considerar que os eletrodos são bons metais, blindando rapidamente as variações de carga (potencial) que podem ocorrer na região central. Isto garante que o potencial externo aplicado cause apenas um deslocamento rígido nos níveis dos eletrodos. Desta forma, a blindagem deve ocorrer já na região central, logo, esta deve conter "pedaços" dos eletrodos, como ilustrado na fig. 2.2 .

Pelo ângulo da mecânica quântica, os hamiltonianos das três regiões (e dos acoplamentos entre elas) devem ser conhecidos. Se considerarmos que os eletrodos são pristinos, isto é, livres de defeitos, e cristalinos, a estrutura de seu hamiltoniano toma a forma mostrada na fig. 2.1. Esta é a primeira aproximação introduzida aqui. Conhecendo os hamiltonianos de cada região, que podem ser calculados por métodos ab initio por exemplo, basta diagonalizar o hamiltoniano total do sistema 
para resolver o problema. Este apresenta a seguinte forma ${ }^{1}$

$$
\begin{aligned}
& H= {\left[\begin{array}{ccc|c|ccc}
\ddots & \ddots & & \vdots & & & \\
\ddots & H_{0} & H_{1} & 0 & & & \\
& H_{1}^{\dagger} & H_{0} & H_{0 M} & & & \\
\hline \cdots & 0 & H_{M 0} & H_{M} & H_{M 0} & 0 & \cdots \\
\hline & & & H_{0 M} & H_{0} & H_{1} & \\
0 & H_{1}^{\dagger} & H_{0} & \ddots \\
& & & & \ddots & \ddots
\end{array}\right] } \\
& {\left[\begin{array}{ccc}
H_{L} & H_{L M} & 0 \\
H_{M L} & H_{M} & H_{M R} \\
0 & H_{R M} & H_{R}
\end{array}\right], } \\
&
\end{aligned}
$$

onde $H_{L(R)}$ é o hamiltoniano do contato esquerdo (direito), $H_{M}$, da região central e $H_{L M}, H_{M L}, H_{R M}$ e $H_{M R}$ são os hamiltonianos que descrevem os acoplamentos entre os contatos e a região central. Aqui estamos assumindo que os eletrodos são formados por blocos, com hamiltoniano $H_{0}$, repetidos periodicamente.

No entanto, como o hamiltoniano total tem dimensão infinita $\left(H_{L / R}\right.$ são semiinfinitos) e o sistema não possui simetria translacional (periodicidade), isto é impossível de fazer, pois não podemos usar o teorema de Bloch. É necessário então recorrer a outros métodos.

Já que, como vimos, é necessário incluir parte dos eletrodos na região central, podemos considerar que os acoplamentos desta com os contatos são iguais aos acoplamentos entre os blocos que compõem os eletrodos (veja a fig. 2.1): $H_{0 M}=H_{M 0}=H_{1}$.

\footnotetext{
${ }^{1}$ No caso da base ser não-ortogonal, teremos uma matriz de superposição $S \neq \mathbf{1}$, com a mesma estrutura do hamiltoniano.
} 


\section{$2.1 \quad$ Transporte de carga}

Vamos agora analisar um modelo simples, no qual uma molécula com um único nível, $\epsilon_{0}$, está conectada a dois eletrodos com espectro contínuo, como mostrado na figura 2.3. Na situação de equilíbrio, os potenciais químicos dos dois contatos são iguais, $\mu_{0}$, coincidindo com a energia de Fermi do sistema, $E_{F}$. A intensidade do acoplamento da molécula com os eletrodos esquerdo e direito são dadas por $\Gamma_{L}$ e $\Gamma_{R}$, respectivamente (unidades de energia). Estes acoplamentos determinam a taxa de transferência de elétrons entre os contatos e a molécula, dados por $\Gamma_{L} / \hbar$ e $\Gamma_{R} / \hbar$. Ao se acoplar com os eletrodos, a densidade de estados da molécula, inicialmente dada por

$$
\delta\left(E-\epsilon_{0}\right)=\lim _{\gamma \rightarrow 0^{+}} \frac{1}{\pi} \frac{\gamma}{\left(E-\epsilon_{0}\right)^{2}+\gamma^{2}},
$$

sofre um alargamento, que pode ser descrito somando uma energia imaginária à $\epsilon_{0}$,

$$
\epsilon=\epsilon_{0}+i \frac{\Gamma_{L}}{2}+i \frac{\Gamma_{R}}{2}
$$

Desta forma, a densidade de estados da molécula na presença dos eletrodos pode ser descrita por uma lorentziana com alargamento $\Gamma_{L}+\Gamma_{R}$,

$$
n(E)=\frac{1}{2 \pi} \frac{\Gamma_{L}+\Gamma_{R}}{\left(E-\epsilon_{0}\right)^{2}+\frac{1}{4}\left(\Gamma_{L}+\Gamma_{R}\right)^{2}} .
$$

Intuitivamente, esperamos que o tempo de vida do estado com energia $\epsilon$ seja inversamente proporcional ao acoplamento $\Gamma_{L}+\Gamma_{R}$ porque se, por exemplo, $\Gamma_{L / R}$ forem pequenos, também o serão as taxas de transferência com os contatos, resultando num tempo de vida maior do elétron no estado. De forma mais rigorosa, a dependência 


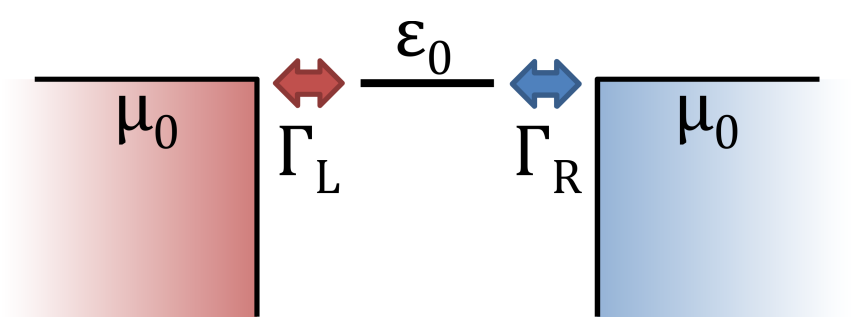

Figura 2.3: Ilustração esquemática de um sistema com um único nível de energia entre dois contatos com espectro contínuo e potencial químico $\mu_{0}$. A intensidade dos acoplamentos com os contatos são dadas por $\Gamma_{L / R}$. Os eletrodos estão em equilíbrio e não há fluxo de corrente.

temporal de um estado com energia $\epsilon=\epsilon_{0}-i \gamma / 2(\gamma$ positivo $)$ é

$$
\begin{aligned}
\exp \left(-i \frac{\epsilon t}{\hbar}\right) & =\exp \left(-i \frac{\left(\epsilon_{0}-i \gamma / 2\right) t}{\hbar}\right) \\
& =\exp \left(-i \frac{\epsilon_{0} t}{\hbar}\right) \exp \left(-\frac{\gamma t}{2 \hbar}\right)
\end{aligned}
$$

Portanto, a probabilidade de achar o elétron no estado é proporcional a $\exp (-\gamma t / \hbar)$, de onde vemos que o tempo de vida médio do elétron no estado é dado por $\hbar / \gamma$.

Quando um potencial $V$ é aplicado, os potenciais químicos esquerdo e direito são deslocados para $\mu_{L}=\mu_{0}+e V / 2$ e $\mu_{R}=\mu_{0}-e V / 2$ (sem perda de generalidade, estamos supondo um deslocamento simétrico resultando em $\mu_{L}>\mu_{R}$ ), possibilitando o fluxo de elétrons do contato esquerdo para o direito através da molécula. Neste caso, há três situações possíveis: se $\epsilon>\mu_{L}$ (fig. 2.4 a) não há elétrons com energia suficiente disponíveis no contato esquerdo que possam tunelar para a molécula; já se $\mu_{L}>\epsilon>\mu_{R}$ (fig. 2.4 b), elétrons nos estados ocupados do contato esquerdo podem fluir para a molécula e desta para os estados desocupados do contato direito, permitindo o fluxo de corrente; finalmente, se $\epsilon<\mu_{L}$ (fig. 2.4c), o princípio de exclusão de Pauli impede os elétrons da molécula de tunelarem para os estados ocupados do contato direito, bloqueando a passagem de corrente. Vemos então, que apenas estados na janela de energia $\left[\mu_{R}, \mu_{L}\right]$ participam do processo de condução.

Para calcular a corrente no caso $\mu_{R}<\epsilon<\mu_{L}$, vamos considerar primeiro a 


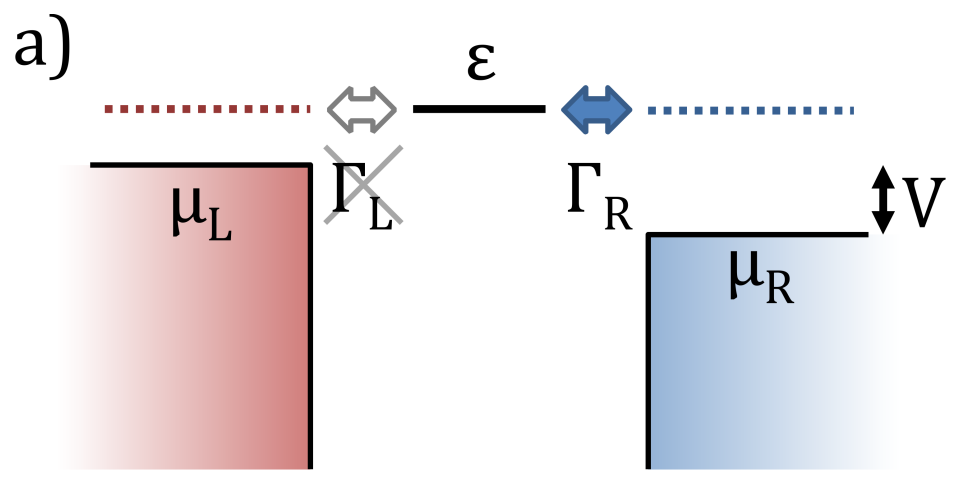

b)

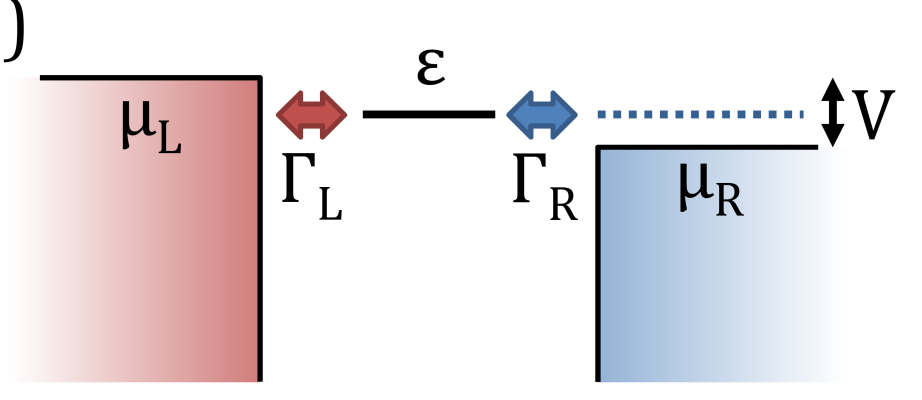

c)

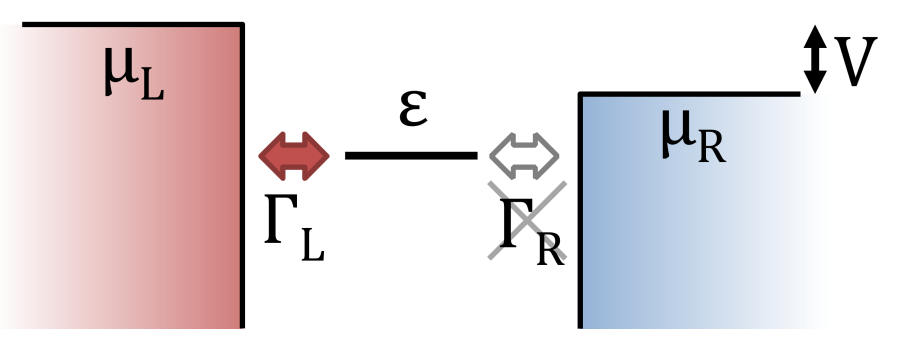

Figura 2.4: Representação esquemática dos regimes de tunelamento em função da energia do nível $\epsilon$ em relação aos potenciais químicos dos contatos. 
ocupação do estado da molécula em equilíbrio com os contatos esquerdo e direito (o fator 2 é devido ao spin),

$$
N_{L}=2 f\left(\epsilon, \mu_{L}\right)
$$

$\mathrm{e}$

$$
N_{R}=2 f\left(\epsilon, \mu_{R}\right)
$$

onde $f$ é a função de Fermi-Dirac, dada por

$$
f(E, \mu)=\frac{1}{\exp \left(\frac{E-\mu}{k_{B} \theta}\right)+1},
$$

onde $k_{B}$ é a constante de Boltzmann e $\theta$ é a temperatura. Quando a molécula está ligada aos dois eletrodos esperamos que sua ocupação, $N$, se situe num valor intermediário entre $N_{L}$ e $N_{R}$. A corrente vinda do contato esquerdo é dada por

$$
I_{L}=\frac{e \Gamma_{L}}{h}\left(N_{L}-N\right)
$$

Enquanto que a corrente fluindo para o contato direito é

$$
I_{R}=\frac{e \Gamma_{R}}{h}\left(N_{R}-N\right)
$$

No estado estacionário as correntes devem ser iguais. Fazendo $I_{L}=I_{M}$ obtemos

$$
I=\frac{2 e}{h} \frac{\Gamma_{L} \Gamma_{R}}{\Gamma_{L}+\Gamma_{R}}\left[f\left(\epsilon, \mu_{L}\right)-f\left(\epsilon, \mu_{R}\right)\right]
$$

Note que com este modelo simples capturamos boa parte da física envolvida no processo de transporte de carga. Vamos analisar agora um formalismo mais elaborado.

No formalismo de Landauer-Büttiker [58, 59, 60], as propriedades de transporte eletrônicas são relacionadas com a probabilidade de transmissão da função de onda do elétron entre os eletrodos, de forma semelhante ao problema de transmissão por 


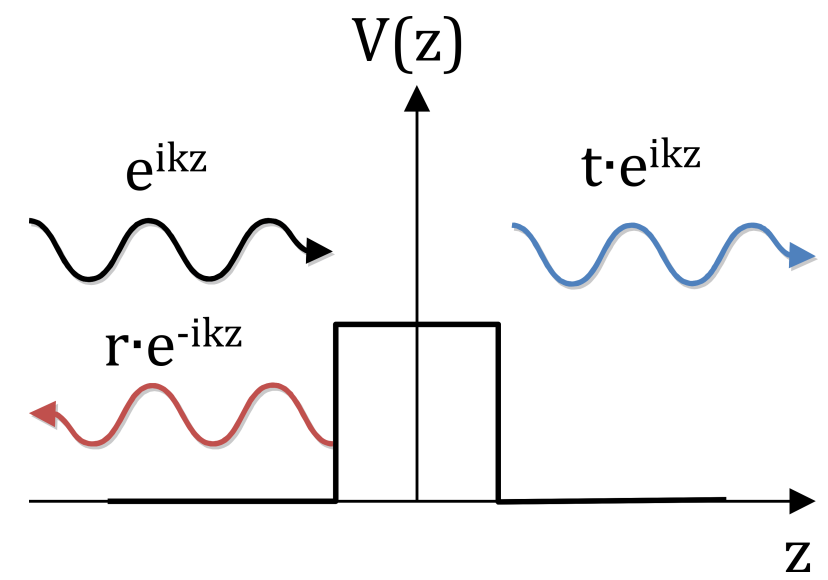

Figura 2.5: No formalismo de Landauer, as propriedades de transporte são relacionadas com as probabilidades de transmissão das funções de ondas dos elétrons, como em um problema de barreira de potencial.

uma barreira de potencial (figura 2.5).

Neste formalismo, a corrente é dada por

$$
I=\frac{2 e}{h} \int T(E)\left[f\left(E, \mu_{L}, \theta_{L}\right)-f\left(E, \mu_{R}, \theta_{R}\right)\right] d E,
$$

onde $T(E)$ é a transmitância de um elétron com energia $E$. De forma semelhante, a condutância do sistema, $G$, é dada por

$$
\begin{aligned}
G & =\frac{2 e^{2}}{h} \int T(E)\left(-\frac{\partial f}{\partial E}\right) d E \\
& =\int \sigma(E) d E
\end{aligned}
$$

onde na última linha definimos a condutância diferencial dependente da energia $\sigma(E)[13]$.

A transmitância, que passa a ser o objeto principal neste formalismo, contendo a informação a respeito das propriedades de transporte, é calculada por meio das funções de Green. 
A Eq. de Schrödinger para o hamiltoniano total do sistema (Eq. 2.2. $\hat{\epsilon}^{2}$

$$
\left[\begin{array}{ccc}
H_{L} & H_{L M} & 0 \\
H_{M L} & H_{M} & H_{M R} \\
0 & H_{R M} & H_{R}
\end{array}\right]\left[\begin{array}{c}
\psi_{L} \\
\psi_{M} \\
\psi_{R}
\end{array}\right]=E\left[\begin{array}{c}
\psi_{L} \\
\psi_{M} \\
\psi_{R}
\end{array}\right]
$$

Contudo, como vimos anteriormente, os hamiltonianos que descrevem os eletrodos, $H_{L / R}$, e os acoplamentos destes com a região central são semi-infinitos, o que torna o problema insolúvel. Voltemos, então, nossa atenção para a função (ou operador) de Green, definida por

$$
(H-E) G=\mathbf{1}
$$

Ou seja,

$$
\left[\begin{array}{ccc}
H_{L}-E & H_{L M} & 0 \\
H_{M L} & H_{M}-E & H_{M R} \\
0 & H_{R M} & H_{R}-E
\end{array}\right]\left[\begin{array}{ccc}
G_{L} & G_{L M} & G_{L R} \\
G_{M L} & G_{M} & G_{M R} \\
G_{R L} & G_{R M} & G_{R}
\end{array}\right]=\left[\begin{array}{ccc}
1 & 0 & 0 \\
0 & 1 & 0 \\
0 & 0 & 1
\end{array}\right]
$$

Tomando as três equações formadas pelas segunda coluna de $G$, obtemos o sistema

$$
\begin{aligned}
\left(H_{L}-E\right) G_{L M}+H_{L M} G_{M} & =0 \\
H_{M L} G_{L M}+\left(H_{M}-E\right) G_{M}+H_{M R} G_{R M} & =1 \\
H_{R M} G_{M}+\left(H_{R}-E\right) G_{R M} & =0,
\end{aligned}
$$

de onde temos,

$$
\begin{aligned}
& 2.19 \Rightarrow G_{L M}=-g_{L} H_{L M} G_{M} \\
& 2.21 \Rightarrow G_{R M}=-g_{R} H_{R M} G_{M},
\end{aligned}
$$

\footnotetext{
${ }^{2}$ Por simplicidade consideramos uma base ortogonal.
} 
onde $g_{L / R}$ são as funções de Green dos contatos, definidas como $\left(H_{L / R}-E\right) g_{L / R}=1$. Ou seja, são as funções de Green dos contatos isolados. Substituindo $G_{L M}$ (Eq. 2.22 ) e $G_{R M}$ (Eq. 2.23) em 2.20), obtemos

$$
H_{M L}\left(-g_{L} H_{L M} G_{M}\right)+\left(H_{M}-E\right) G_{M}+H_{M R}\left(-g_{R} H_{R M} G_{M}\right)=1 .
$$

Rearrajando os termos da equação acima, temos

$$
\left[H_{M}-E-\left(H_{M L} g_{L} H_{L M}\right)-\left(H_{M R} g_{R} H_{R M}\right)\right] G_{M}=1
$$

E assim, como resultado final, obtemos

$$
G_{M}(E)=\left[\left(H_{M}+\Sigma_{L}(E)+\Sigma_{R}(E)\right)-E\right]^{-1},
$$

onde definimos as autoenergias

$$
\begin{aligned}
& \Sigma_{L}(E)=-H_{M L} g_{L}(E) H_{L M} \\
& \Sigma_{R}(E)=-H_{M R} g_{R}(E) H_{R M} .
\end{aligned}
$$

Portanto, o efeito dos eletrodos sobre a região central consiste na adição das autoenergias $\Sigma_{L}$ e $\Sigma_{R}$ sobre o hamitoniano $H_{M}$. Note também que apesar das matrizes $H_{M L(R)}, g_{L(R)}$ e $H_{L(R) M}$ serem semi-infinitas, o produto delas não o é devido à sua estrutura (veja a Eq. 2.2).

Agora que conhecemos a função de Green da região central podemos calcular a transmitância do sistema, que no formalismo de funções de Green fora do equilíbrio [59, 60], é dada por

$$
T(E)=\operatorname{Tr}\left\{\Gamma_{L} G_{M}^{\dagger} \Gamma_{R} G_{M}\right\}
$$

onde as matrizes $\Gamma_{L(R)}$, que descrevem o acoplamento com os eletrodos, são dadas 
por

$$
\begin{aligned}
\Gamma_{L(R)}(E) & =i\left[\Sigma_{L(R)}(E)-\Sigma_{L(R)}^{\dagger}(E)\right] \\
& =-2 \operatorname{Im}\left\{\Sigma_{L(R)}(E)\right\} .
\end{aligned}
$$

Este formalismo está implementado no código TRANSAMPA [14, 15], que usamos para realizar este trabalho.

\subsection{Transporte de calor}

Em uma rede cristalina o transporte de calor é realizado de duas formas: através das vibrações da própria rede, isto é, por fônons, e através dos elétrons, que também carregam calor. Portanto, a condutância térmica total é dada pela soma das contribuições eletrônica, $\kappa_{e l}$, e da rede, $\kappa_{p h}$ (usamos o subscrito $p h$ do inglês phonon):

$$
\kappa=\kappa_{p h}+\kappa_{e l}
$$

Da mesma forma, a corrente de calor total é

$$
I_{Q}=I_{Q, p h}+I_{Q, e l}
$$

No formalismo de Landauer-Büttiker a corrente de calor fonônica é [61, 62, 59]

$$
I_{Q, p h}=\frac{1}{h} \int T_{p h}(E) E\left[N\left(E, \Theta_{L}\right)-N\left(E, \Theta_{R}\right)\right] d E
$$

onde $T_{p h}(E)$ é a probabibilidade de transmissão (i.e., a transmitância) de um fônon com energia $E, \Theta_{L(R)}$ é a temperatura do reservatório de fônons esquerdo (direito) 
e $N(E, \Theta)$ é a função de distribuição de Bose-Einstein,

$$
N(E, \Theta)=\frac{1}{\exp \left(\frac{E}{k_{B} \Theta}\right)-1} .
$$

Da mesma forma que no caso da transmitância eletrônica, poderíamos recorrer ao formalismo de funções de Green fora do equilíbrio para obter $T_{p h}$ (veja as Refs. [63, 62]). Contudo, se considerarmos que a região central forma uma ligação mecânica fraca, podemos modelar a mesma como uma mola [60], cuja constante de força pode vir a ser determinada por métodos de primeiros princípios. Esta suposição é razoável se a região central é composta por uma molécula ou um material mole (em comparação aos contatos), por exemplo. Nesta aproximação, a corrente de calor fonônica é dada por

$$
I_{Q, p h}=\frac{2 \pi \mathcal{K}^{2}}{\hbar} \int n_{L}(E) n_{R}(E) E\left[N\left(E, \Theta_{L}\right)-N\left(E, \Theta_{R}\right)\right] d E,
$$

onde $\mathcal{K}=A Y / \ell$ é a rigidez da região central ( $Y$ é o módulo de Young, $A$ é a área de seção transversal e $\ell$, o comprimento) e $n_{L(R)}(E)$ é a densidade de estados (de fônons) do contato esquerdo (direito). Estas grandezas $\left(\mathcal{K}, n_{L(R)}\right)$ são mais fáceis de se obter do que $T_{p h}$.

A corrente de calor eletrônica também pode ser calculada dentro do formalismo de Landauer, no qual, de forma semelhante à corrente eletrônica (Eq. 2.13), temos [59, 60]

$$
I_{Q, e l}=\frac{2}{h} \int T(E)(E-\mu)\left[f\left(E, \mu_{L}, \theta_{L}\right)-f\left(E, \mu_{R}, \theta_{R}\right)\right] d E .
$$

Esta expressão é praticamente idêntica à usada para calcular a corrente eletrônica $I$ (Eq. 2.13), sendo as únicas diferenças são o fator $(E-\mu)$ na integral ( $\mu$ é o potencial químico na região central), que contabiliza a energia carregada pelo elétrons e, obviamente, a constante na frente da integral. Assim, o nível de dificuldade de calcular $I_{Q, e l}$ é o mesmo de calcular $I$. Por outro lado, para obter a corrente de calor 
fonônica seria necessário calcular $T_{p h}$ ou, na aproximação de ligação mecânica fraca, as densidades de estados de fônons dos contatos, $n_{L}$ e $n_{R}$, e a rigidez $\mathcal{K}$, o que eleva a dificuldade dos cálculos em ambos os casos.

\subsection{Efeitos Termoelétricos}

Efeitos termoelétricos relacionam campos elétricos com gradientes de temperatura, ou seja, são efeitos de conversão entre energia elétrica e energia térmica. Estes pertencem a uma gama de efeitos mais ampla, os efeitos termomagnetoelétricos [6, 7].

Há três efeitos que formam a família dos chamados efeitos termoelétricos [7]:

- O efeito Seebeck, que descreve o surgimento de uma diferença de potencial em resposta a uma diferença de temperatura aplicada no material.

- O efeito Peltier, que é o inverso do efeito Seebeck, acontece quando há o desenvolvimento de uma diferença de temperatura em função de um diferença de potencial aplicada.

- O efeito Thomson, que descreve o aquecimento ou resfriamento (incluindo o efeito Joule) de um material com um gradiente de temperatura. Este efeito não será tratado neste trabalho.

Assim, estes efeitos podem ser aplicados para gerar energia e recuperar excedentes de calor [8, 9] (Seebeck), e como refrigeradores ou aquecedores [64, 9, 8] (Peltier).

\subsubsection{Efeito Seebeck}

Em 1821, Thomas Johann Seebeck observou que ao juntar duas placas de materiais diferentes, bismuto e cobre, e esquentar uma das junções, ocorria a deflexão da agulha imantada de uma bússola (veja a fig. 2.6). Esta deflexão era causada pelo 

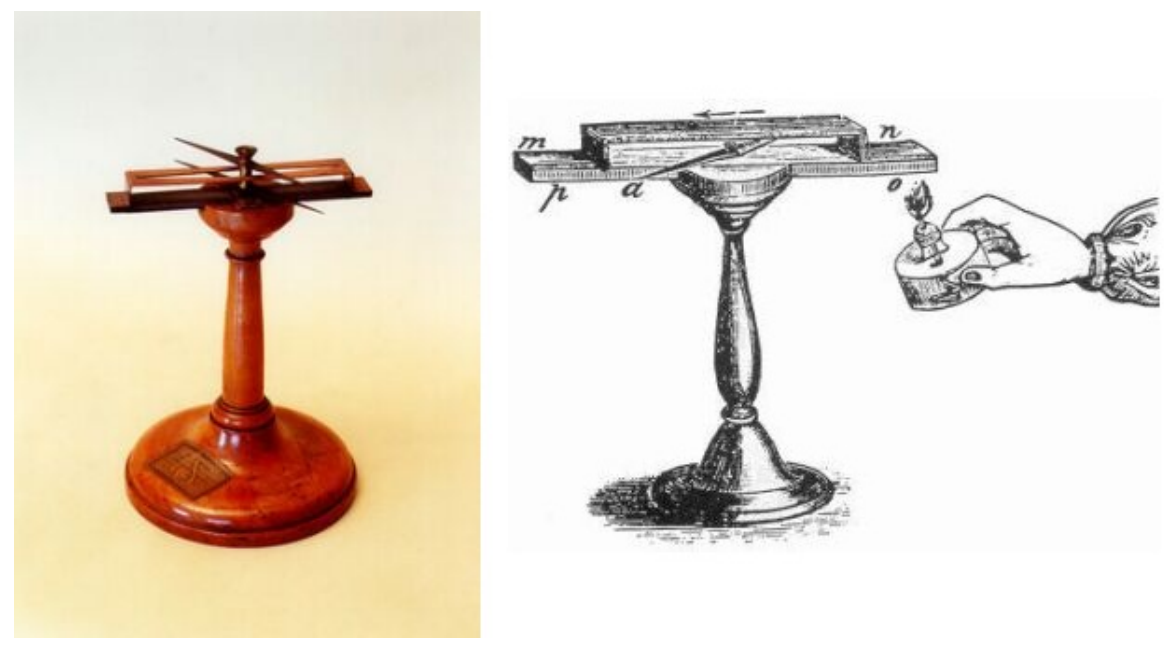

Figura 2.6: Foto do equipamento usado por Seebeck (à esquerda) e seu esquema de uso (à direita). Ao esquentar uma das extremidades de um circuito formado por metais diferentes, Seebeck observou que uma agulha imantada era defletida.

campo magnético gerado pela corrente que surgia no circuito devido à diferença de temperatura entre as junções. Este efeito, que foi chamado de Seebeck, trata da conversão de energia térmica em energia elétrica, ou mais especificamente, do surgimento de um campo elétrico devido à uma diferença de temperatura entre as extremidades de um material.

O mecanismo físico por trás deste efeito é conceitualmente simples: a função de distribuição eletrônica (de Fermi-Dirac) na extremidade quente apresenta uma população maior nos níveis com energia acima de $E_{F}$ do que a extremidade fria. Assim, sempre há mais elétrons difundindo do lado quente para o lado frio do que vice-versa, o que gera uma diferença de potencial entre as extremidades, como exemplificado no desenho da figura 2.7. Se o circuito for aberto, o sistema atinge o equilíbrio devido ao acumulo de carga nas extremidades. Mas se for fechado, os elétrons podem continuar fluindo na chamada termocorrente. Este princípio é o que está por trás dos geradores termoelétricos.

O coeficiente Seebeck, $S$, mede a razão entre a diferença de temperatura aplicada nas extremidades do material, $\Delta \theta$, e a termovoltagem, $\Delta V$, desenvolvida em 


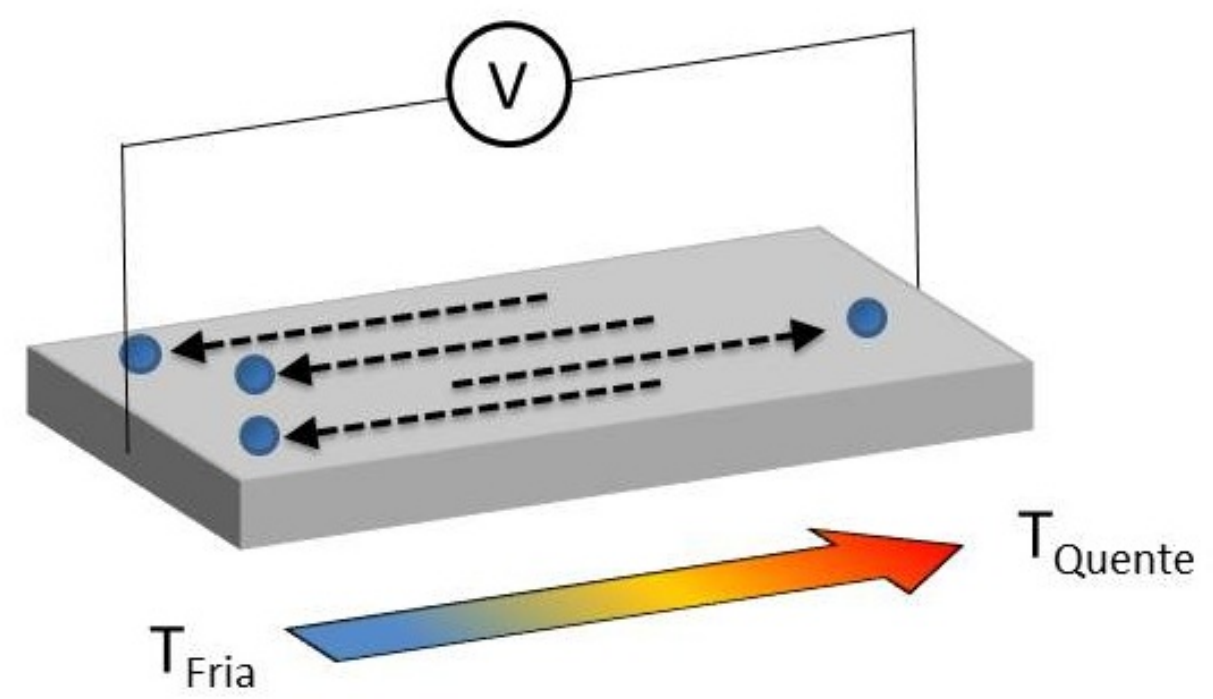

Figura 2.7: Ilustração esquemática do efeito Seebeck. Os elétrons da extremidade quente, que são mais energéticos, difundem para a extremidade fria numa taxa maior do que a dos elétrons da extremidade fria, gerando uma diferença de potencial.

consequência,

$$
\Delta V=S \Delta \theta
$$

Experimentalmente, $S$ pode ser definido como a diferença de potencial externo aplicada necessária para anular a termovoltagem. Desta forma, quando a corrente for nula, temos

$$
S=\left.\frac{V_{e x t}}{\Delta \theta}\right|_{I=0}=-\left.\frac{\Delta V}{\Delta \theta}\right|_{I=0} .
$$

Como vimos na seção 2.1, no formalismo de Landauer-Büttiker, a corrente em um sistema de duas pontas ligado a reservatórios com potenciais químicos e temperaturas $\mu_{L}, \theta_{L}$ e $\mu_{R}, \theta_{R}$ é dada por (Eq. 2.13)

$$
I\left(\mu_{L}, \theta_{L} ; \mu_{R}, \theta_{R}\right)=\frac{e}{\pi \hbar} \int T(E)\left[f\left(E, \mu_{L}, \theta_{L}\right)-f\left(E, \mu_{R}, \theta_{R}\right)\right] d E
$$

Aplicando a expansão de Sommerfeld [6] para o potencial eletroquímico e para a temperatura obtemos a corrente no sistema na existência de pequenas diferenças de 
potencial, $\Delta \mu=e \Delta V$, e temperatura, $\Delta \theta$ [65, 66],

$$
I=\frac{e^{2}}{\pi \hbar} T\left(E_{F}\right) \Delta V-\left.\frac{e}{\pi \hbar} \frac{\pi^{2} k_{B}^{2}}{3} \bar{\theta} \frac{\partial T}{\partial E}\right|_{E_{F}} \Delta \theta
$$

onde $\bar{\theta}$ é a temperatura média entre os contatos.

Fazendo $I=0$ na Eq. 2.41 obtemos uma expressão para a termovoltagem, isto é, para a voltagem gerada pela diferença de temperatura $\Delta \theta$,

$$
\begin{aligned}
\Delta V & =\left.\frac{\pi^{2} k_{B}^{2}}{3 e} \bar{\theta} \frac{1}{T} \frac{\partial T}{\partial E}\right|_{E_{F}} \Delta \theta \\
& =\left.\frac{\pi^{2} k_{B}^{2}}{3 e} \bar{\theta} \frac{\partial \ln (T)}{\partial E}\right|_{E_{F}} \Delta \theta .
\end{aligned}
$$

Logo, o coeficiente Seebeck resultante, também conhecido como fórmula de Mott [66], pode ser escrito como:

$$
S=-\frac{\Delta V}{\Delta \theta}=-\left.\frac{\pi^{2} k_{B}^{2}}{3 e} \bar{\theta} \frac{\partial \ln (T)}{\partial E}\right|_{E_{F}} .
$$

Vemos, assim, que $S$ é proporcional à derivada da transmitância e, portanto, devemos esperar picos no coeficiente Seebeck quando, (i) houver uma mudança na transmitância devido à inclusão (ou remoção) de um canal de condução e na existência de estados no nível de Fermi que (ii) sejam ressonantes no processo de transporte (nestes dois casos há um aumento da transmitância) [66, 67] ou que (iii) sejam localizados e causem espalhamento numa faixa estreita de energia (diminuindo a transmitância). Vale lembrar que a expressão acima (Eq. 2.43 para $S$, por ser derivada da expansão de Sommerfeld, é válida apenas para temperaturas $s^{3}$ e diferenças de temperatura e voltagem baixas, e na ausência de estados ressonantes no nível de Fermi.

Considere, agora, uma variação na corrente produzida pela presença de pequenas variações no potencial eletroquímico e na temperatura, $\Delta \mu=e \Delta V / 2$ e $\Delta \theta^{\prime}=\Delta \theta / 2$,

\footnotetext{
${ }^{3} k_{B} \theta$ deve ser a menor escala de energia (veja a Ref. [66]).
} 
isto é

$$
\Delta I=I\left(\mu_{L}+\Delta \mu, \theta_{L}+\Delta \theta^{\prime} ; \mu_{R}-\Delta \mu, \theta_{R}-\Delta \theta^{\prime}\right)-I\left(\mu_{L}, \theta_{L} ; \mu_{R}, \theta_{R}\right) .
$$

Ao invés de aplicar a expansão de Sommerfeld na expressão acima (Eq. 2.44), podemos fazer a expansão em série de Taylor da função de Fermi-Dirac. Considerando apenas os termos de primeira ordem, obtemos

$$
\begin{aligned}
f\left(E, \mu_{i}+\Delta \mu, \theta_{i}+\Delta \theta^{\prime}\right) & =f\left(E, \mu_{i}, \theta\right)+\frac{\partial f}{\partial \mu} \Delta \mu+\frac{\partial f}{\partial \theta} \Delta \theta^{\prime} \\
& =f\left(E, \mu_{i}, \theta\right)-\frac{\partial f}{\partial E} \Delta \mu-\frac{\partial f}{\partial E}(E-\mu) \frac{\Delta \theta^{\prime}}{\theta_{i}+\Delta \theta^{\prime}}
\end{aligned}
$$

onde $i=L, R$. Desta forma, a expressão para a variação da corrente na presença de $\Delta V$ e $\Delta \theta$ é dada por

$$
\begin{aligned}
\Delta I & =\frac{e^{2} \Delta V}{2 \pi \hbar} \int T(E)\left(-\frac{\partial f_{L}}{\partial E}-\frac{\partial f_{R}}{\partial E}\right) d E \\
& +\frac{e \Delta \theta}{2 \pi \hbar \bar{\theta}} \int T(E)\left[-\frac{\partial f_{L}}{\partial E}\left(E-\mu_{L}\right)-\frac{\partial f_{R}}{\partial E}\left(E-\mu_{R}\right)\right] d E
\end{aligned}
$$

onde $f_{L(R)} \equiv f\left(E, \mu_{L(R)}, \theta_{L(R)}\right)$ e $\bar{\theta}=\left(\theta_{L}+\theta_{R}\right) / 2$ é a temperatura média dos eletrodos.

Definindo o termo

$$
K_{n}=\int T(E)\left(-\frac{\partial f}{\partial E}\right)(E-\mu)^{n} d E
$$

usado para simplificar a notação, e substituindo em 2.46 temos

$$
\Delta I=\frac{e^{2} \Delta V}{2 \pi \hbar}\left(K_{0}^{L}+K_{0}^{R}\right)+\frac{e \Delta \theta}{2 \pi \hbar \bar{\theta}}\left(K_{1}^{L}+K_{1}^{R}\right)
$$

onde o sobrescrito $L(R)$ em $K_{n}^{L(R)}$ indica o uso de $\mu=\mu_{L(R)}$ em 2.47). Fazendo 
$\Delta I=0$ obtemos uma nova expressão para o coeficiente Seebeck,

$$
S=-\frac{1}{e \bar{\theta}} \frac{K_{1}^{L}+K_{1}^{R}}{K_{0}^{L}+K_{0}^{R}} .
$$

No limite de baixas temperaturas e voltagens (regime linear), no qual $\mu_{L} \approx \mu_{R} \approx \mu$ e $\theta_{L} \approx \theta_{R} \approx \bar{\theta}$, podemos escrever

$$
S=-\frac{1}{e \bar{\theta}} \frac{K_{1}}{K_{0}}
$$

Esta última expressão, por envolver um número menor de aproximações que a derivada através da expansão de Sommerfeld (Eq. (2.43)) é mais precisa e pode ser usada para temperaturas maiores [66]. É importante notar que o numerador da Eq. 2.50 , o termo $K_{1}$, que é escrito como

$$
K_{1}=\int T(E)\left(-\frac{\partial f}{\partial E}\right)(E-\mu) d E
$$

é uma medida da assimetria da transmitância $T(E)$ em torno de $\mu\left(=E_{F}\right)$. Ou seja, para que $S$ seja diferente de zero é necessário que $T(E)$ seja assimétrica em torno do nível de Fermi (o intervalo de energias relevante é definido por $-\partial f / \partial E$ ). Isto também pode ser visto mais claramente na fórmula de Mott (Eq. 2.43)), lembrando que $\partial \ln (T) / \partial E=(1 / T) \partial T / \partial E$.

Considerando que as extremidades do material sujeito ao gradiente de temperatura (Fig. 2.7) estão em equilíbrio local, a distribuição de energia dos elétrons será dada pela distribuição de Fermi-Dirac (Eq. (2.9)). Desta forma, o desbalanço entre a quantidade de portadores termicamente excitados entre os lados quente e frio permite que elétrons, $e^{-}$, e buracos, $h^{+}$, possam fluir do lado quente para o lado frio do material (veja a Fig. 2.8). No entanto, a probabilidade de transmissão dos portadores será dada por $T(E)$. Assim, se considerarmos uma transmitância tipicamente metálica, com $T(E)$ constante (Fig. 2.8 a), teremos $e^{-}$e $h^{+}$fluindo à 
a)

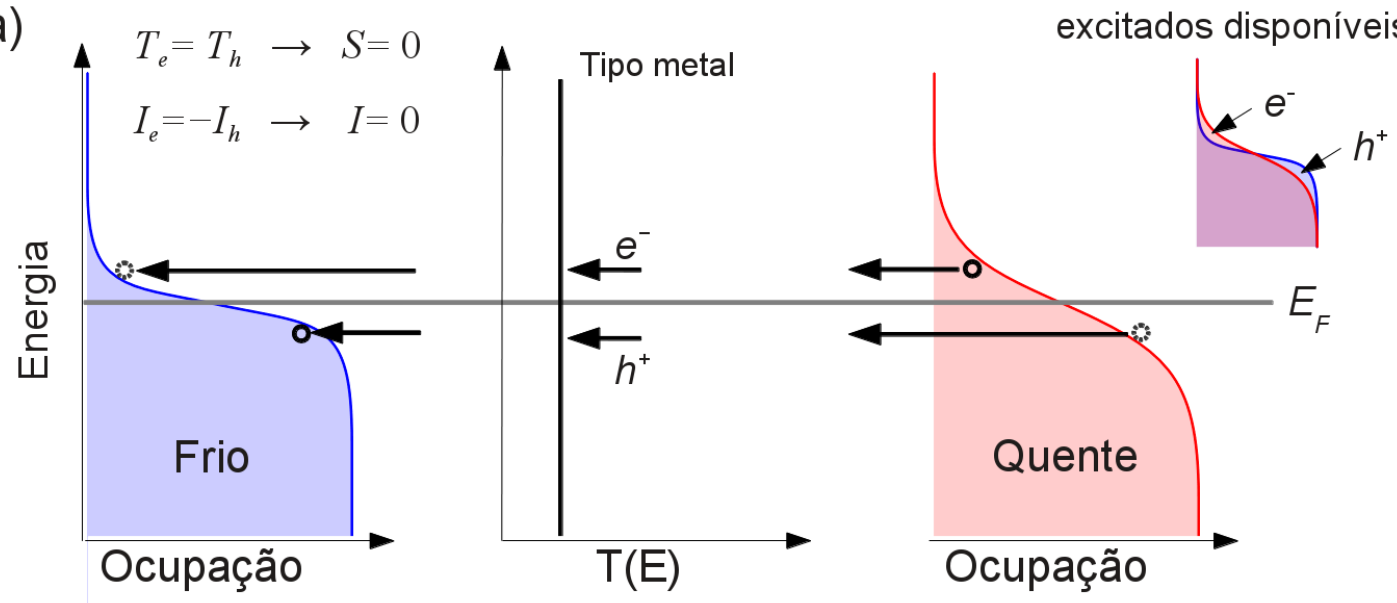

b)

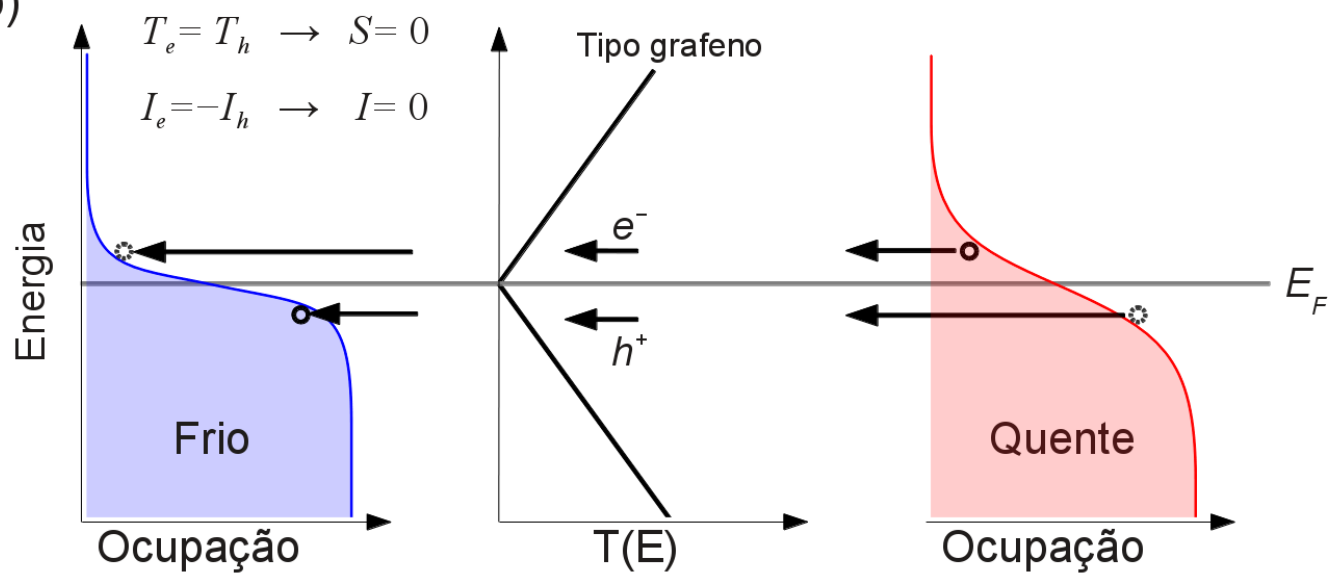

c)

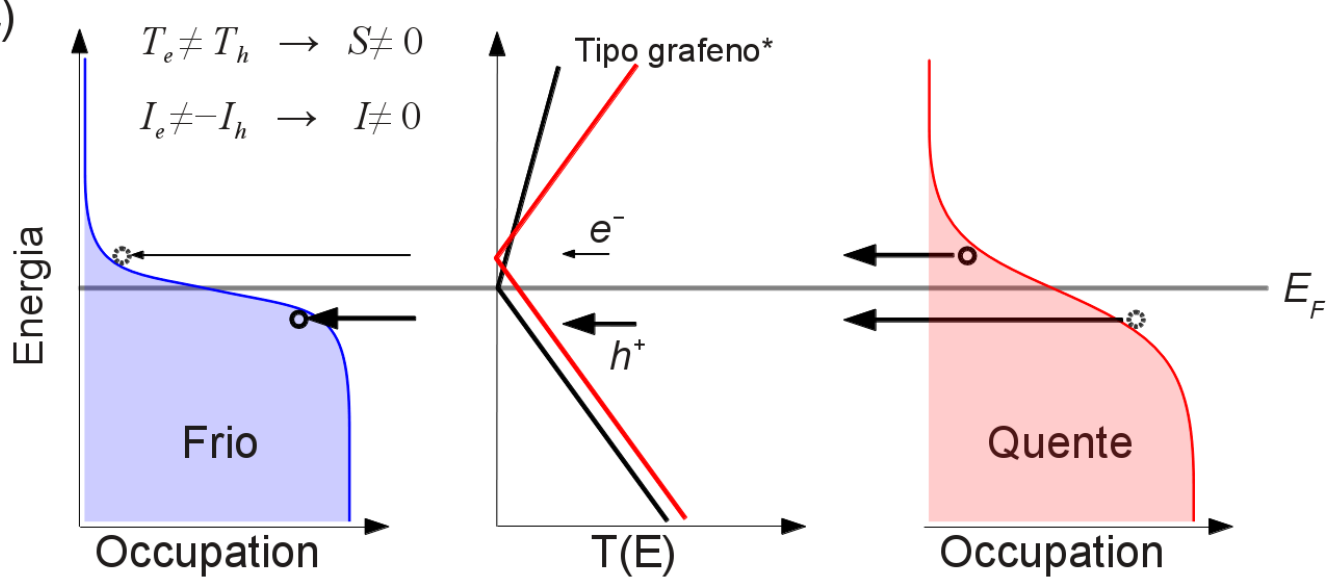

Figura 2.8: Diagrama esquemático do mecanismo do efeito Seebeck considerando uma transmitância, $T(E)$, tipo (a) metal, (b) do grafeno, e (c) grafeno deslocada (linha vermelha) ou onde a inclinação de um dos ramos foi alterada (linha preta). 
mesma taxa do lado quente para o frio e, portanto, o termo $K_{1}$ (Eq. (2.51) no numerador de $S$ se anularát Considerando uma transmitância tipo a do grafeno, como mostrada na Fig. 2.8b (note a semelhança de $T(E)$ com o gráfico da condutividade na Fig. 1.4a), apesar de ter uma variação em $T(E)$, esta é simétrica em torno de $E_{F}$ e, novamente, o fluxo de $e^{-}$e $h^{+}$se cancela mutuamente, proporcionando $S=0$. Agora, se for possível tornar a transmitância do grafeno assimétrica, aplicando um potencial de gate (veja a Fig. 1.4a) ou introduzindo dopantes (como veremos no Cap. 5), por exemplo, como ilustrado na Fig. 2.8c, passaremos a ter um fluxo líquido de portadores termicamente excitados do lado quente para o lado frio, resultando em um coeficiente Seebeck não nulo.

Outra característica importante a ser notada na expressão para o coeficiente Seebeck (Eq. (2.50), é que o sinal de $S$ permite identificar a natureza dos portadores de carga envolvidos. Como mostrado na Fig. 2.9, o sinal do termo $K_{1}$, e portanto, o sinal de $S$ (o termo $K_{0}$ no denominador da Eq. (2.50) é sempre positivo) é sempre negativo para $e^{-}\left(E>E_{F}\right)$ e sempre positivo para $h^{+}\left(E<E_{F}\right)$. Vemos assim, que uma medida do coeficiente Seebeck pode determinar se o material está dopado tipo $n$ ou tipo $p$.

\footnotetext{
${ }^{4}$ Nos metais, de forma geral, $S$ é muito pequeno, da ordem de poucos $\mu \mathrm{V} / \mathrm{K}$. Já em semicondutores, costuma ser da ordem de centenas de $\mu \mathrm{V} / \mathrm{K}$, tipicamente.
} 


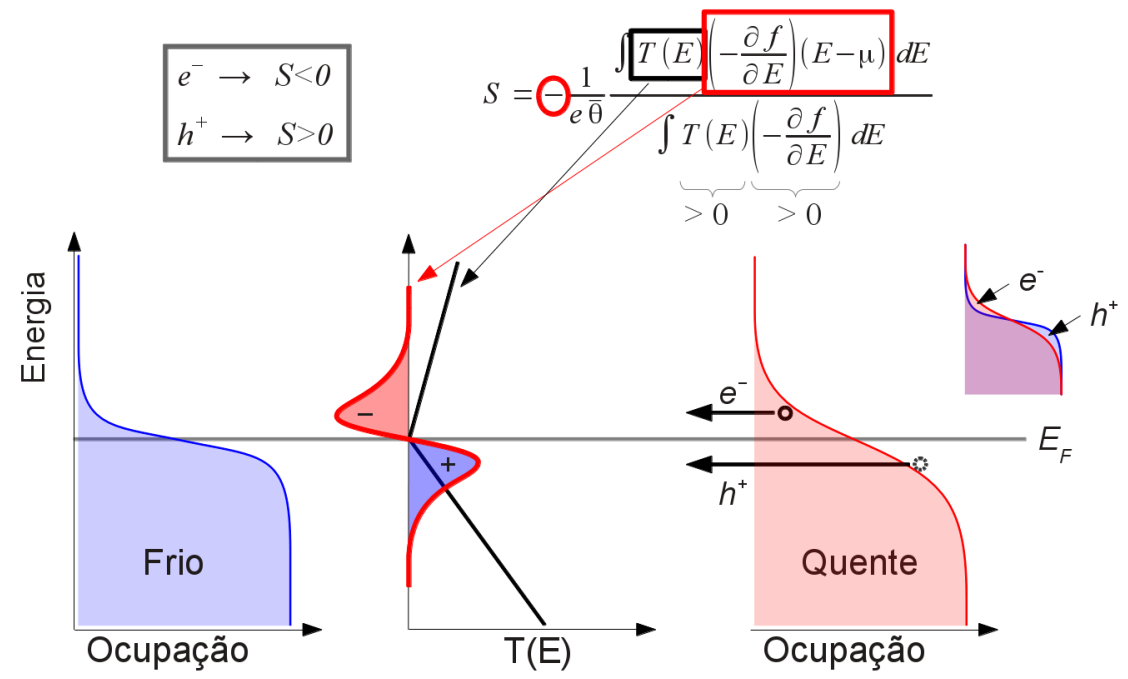

Figura 2.9: Diagrama esquemático do mecanismo do efeito Seebeck considerando uma transmitância, $T(E)$, tipo grafeno. Note que elétrons $\left(E>E_{F}\right)$ sempre dão uma contribuição negativa para o coeficiente Seebeck, $S$, enquanto que buracos $\left(E<E_{F}\right)$, contrariamente, sempre dão uma contribuição positiva.

\subsubsection{Efeito Peltier}

O efeito Peltier, descoberto por Jean Charles Athanase Peltier em 1834, é basicamente o contrário do efeito Seebeck. Peltier observou que uma junção formada por metais diferentes (novamente bismuto e cobre) esquentava ao ser percorrida por uma corrente elétrica em um determinado sentido e esfriava quando a corrente fluía no sentido contrário. Assim como no caso do efeito Seebeck, o mecanismo físico pelo qual o efeito acontece é simples: os portadores de carga carregam diferentes quantidades de calor em cada material. Logo, ao passar pela junção os portadores podem passar a carregar mais calor, esfriando a junção devido ao calor que é levado embora, ou podem carregar menos, esquentando a junção por causa do calor que se acumula (veja a fig. 2.10). Este efeito descreve a conversão de energia elétrica em energia térmica, sendo o princípio usado pelos refrigeradores (ou aquecedores) termoelétricos.

O coeficiente Peltier, $\Pi$, define a proporcionalidade entre a corrente eletrônica no 


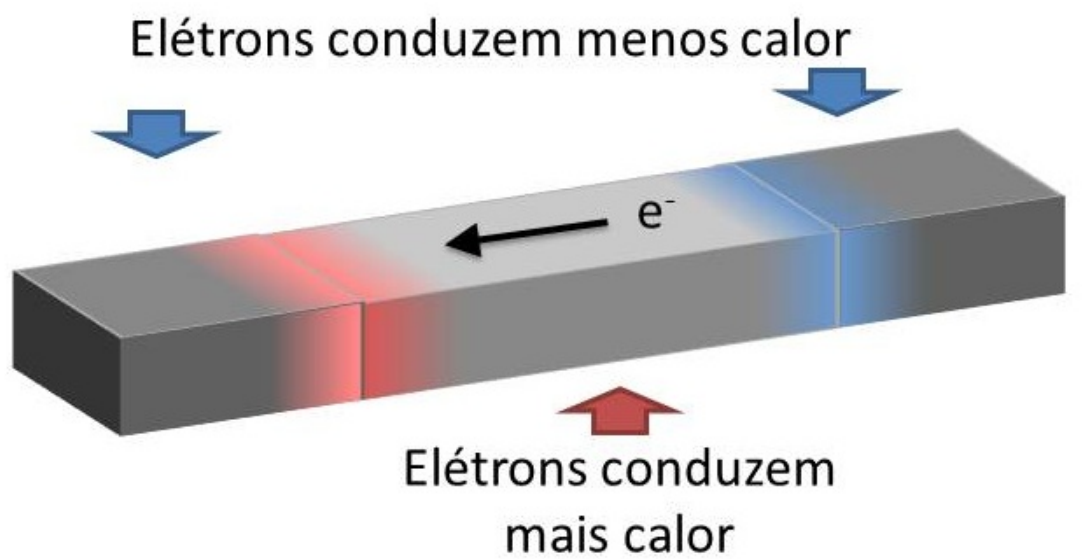

Figura 2.10: Ilustração esquemática do efeito Peltier. Ao passar pelas junções, os portadores passam a carregar mais ou menos calor, esfriando ou esquentando a respectiva junção. O fluxo de elétrons é mantido por uma bateria externa, não mostrada na figura.

material e o fluxo de calor (eletrônico) gerado quando o gradiente de temperatura é nulo,

$$
I_{Q}=\Pi I \quad \text { para } \Delta \theta=0
$$

Então temos que П é dado por (veja as eqs. 2.13 e 2.37)

$$
\begin{aligned}
\Pi & =\left.\frac{I_{Q}}{I}\right|_{\Delta \theta=0} \\
& =\frac{1}{e} \frac{\int T(E)\left[f\left(E, \mu_{L}, \theta_{L}\right)-f\left(E, \mu_{R}, \theta_{R}\right)\right](E-\mu) d E}{\int T(E)\left[f\left(E, \mu_{L}, \theta_{L}\right)-f\left(E, \mu_{R}, \theta_{R}\right)\right] d E} .
\end{aligned}
$$

Aplicando o mesmo procedimento usado para calcular o coeficiente Seebeck (Eq. 2.49, ou seja, expandindo as funções de Fermi-Dirac em séries de Taylor, obtemos (lembrando que devemos fazer $\Delta \theta=0$ )

$$
\begin{aligned}
\Pi & =-\frac{1}{e} \frac{K_{1}^{L}+K_{1}^{R}}{K_{0}^{L}+K_{0}^{R}} \\
& =S \theta .
\end{aligned}
$$

Este último resultado coincide com a segunda lei de Thomson (ou Kelvin), que foi 
proposta em 1854, baseada em observações experimentais, e demonstrada em 1931 por Lars Onsanger [9], demonstrando que a abordagem aqui usada para o cálculo das propriedades termoelétricas satisfaz conhecidas relações fundamentais, confirmadas experimentalmente.

\subsubsection{Dispositivos termoelétricos}

Na prática, os dispositivos termoelétricos são construídos por unidades compostas por segmentos de semicondutores do tipo-n e do tipo-p ligados por contatos metálicos, como mostrado na figura 2.11. Com este tipo de desenho, os portadores de carga em cada segmento do dispositivo (elétrons para o semicondutor tipo-n e buracos para o tipo-p) se movem no mesmo sentido, consequentemente, conduzindo calor no mesmo sentido também.

Nos dispositivos termoelétricos (fig. 2.11k), um grande número destas unidades são ligadas em série, aumentando a voltagem operacional e espalhando o fluxo de calor. Note que o mesmo dispositivo pode ser usado tanto como gerador, refrigerador ou aquecedor.

Dispositivos termoelétricos possuem as vantagens de serem pequenos, apresentarem resposta rápida e, por serem dispositivos de estado sólido e não possuir partes móveis ou fluidos, de terem uma alta confiabilidade, com pouca necessidade de manutenção [8, 9, 12]. No entanto, sua aplicabilidade tem encontrado uma limitação na sua baixa eficiência. Assim, dispositivos termoelétricos são empregados em aplicações onde confiabilidade, tamanho e resposta rápida são mais importantes do que eficiência.

\subsubsection{Figura de Mérito adimensional $Z \theta$}

Os dispositivos termoelétricos são, em última análise, máquinas térmicas, e portanto devem obedecer as leis da termodinâmica. Assim, podemos calcular a eficiência 


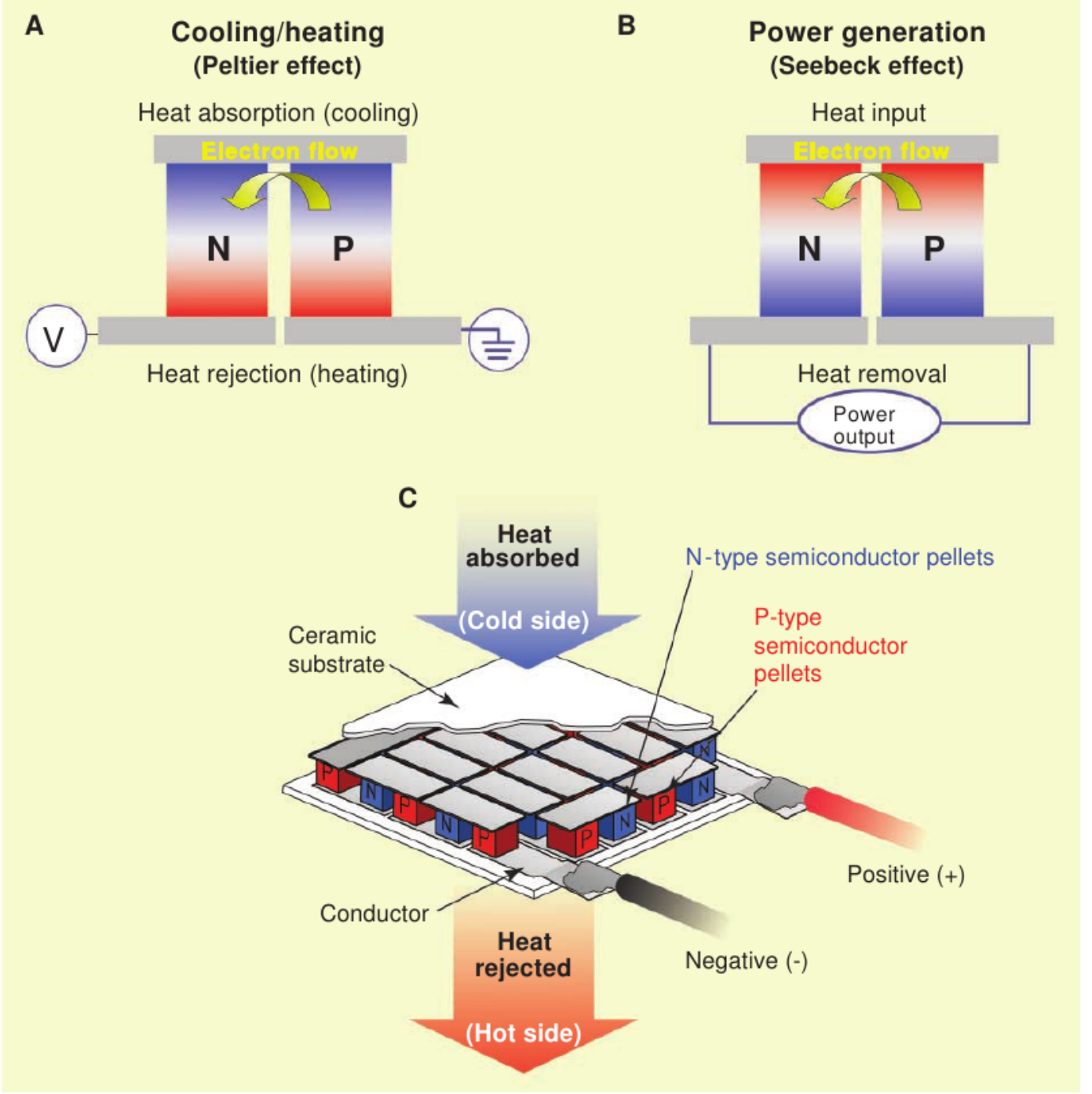

Figura 2.11: Ilustração esquemática de dispositivos termoelétricos típicos. (a) Refrigerador (ou aquecedor) por efeito Peltier. (b) Gerador elétrico por efeito Seebeck. (c) Arranjo típico dos dispositivos. Figura retirada da Ref. [8]. 
máxima teórica destes dispositivos. No caso de geradores termoelétricos a eficiência máxima é dada por [68, 63]

$$
\eta_{\max }=\frac{\theta_{H}-\theta_{C}}{\theta_{H}}\left(1-\frac{1+\theta_{C} / \theta_{H}}{\sqrt{1+Z \theta}+\theta_{C} / \theta_{H}}\right),
$$

onde $\theta_{H(C)}$ é a temperatura da fonte quente (fria) e $Z \theta$ é a chamada figura de mérito adimensional, com $Z$ definido por

$$
Z=\frac{G S^{2}}{\kappa}=\frac{G S^{2}}{\kappa_{p h}+\kappa_{e l}}
$$

sendo $G$ a condutância eletrônica (Eq. 2.14), $S$ o coeficiente de Seebeck (Eq. 2.50) e $\kappa$ a condutância térmica (Eq. 2.32). Da expressão para $\eta_{\max }$ (Eq. 2.56), vemos que quanto maior for o valor de $Z \theta$ (que a princípio é ilimitado) mais perto $\eta_{\max }$ estará do limite de eficiência de Carnot, $\left(\theta_{H}-\theta_{C}\right) / \theta_{H}$ (quando $Z \theta=\infty, \eta_{\max }=\eta_{\text {Carnot }}$ ). Na figura 2.12, mostramos um gráfico de $\eta_{\max }$ para vários valores de $Z \theta$.

Da mesma forma, para um refrigerador termoelétrico, temos uma eficiência máxima semelhante à do gerador [68, 63],

$$
\eta_{\max }=\frac{\theta_{C}}{\theta_{H}-\theta_{C}}\left(1-\frac{1+\theta_{H} / \theta_{C}}{\sqrt{1+Z \theta}+1}\right)
$$

Também neste caso, quanto maior for $Z \theta$, mais próxima do limite teórico será sua eficiência máxima.

Vemos assim, que a figura de mérito $Z \theta$ constitui um parâmetro simples que determina a eficiência termoelétrica de um dado material ou dispositivo. Para otimizar $Z$, ou seja, conseguir um valor o mais alto possível, devemos otimizar simultaneamente $G$ e $S$, que devem ser os maiores possíveis e $\kappa_{e l}$ e $\kappa_{p h}$, que devem ser os menores possíveis. Isto, no entanto, não é uma tarefa simples pois elas não são totalmente independentes. Por exemplo, as condutâncias $G$ e $\kappa_{e l}$ estão relacionadas pela lei de Wiedemann-Franz, sendo uma proporcional à outra. 


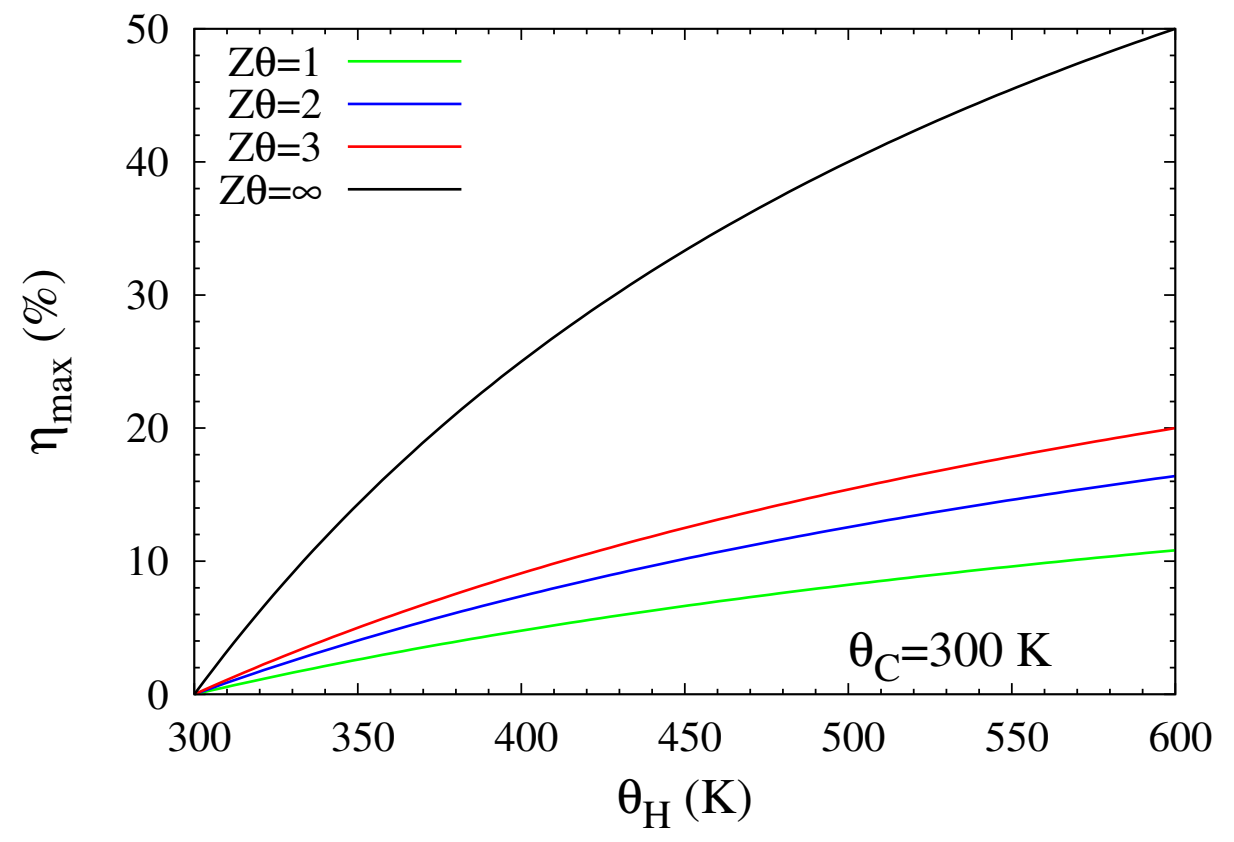

Figura 2.12: Eficiência máxima de um gerador termoelétrico para vários valores de $Z \theta$, considerando a temperatura da fonte fria $\theta_{C}=300 \mathrm{~K}$.

Vamos agora considerar uma aproximação que facilita muito o cálculo da figura de mérito (veja a discussão no final da seção 2.2. . No caso de materiais que possuem um mobilidade eletrônica muito alta, e portanto, uma condutância eletrônica $G$ alta, a condutividade térmica eletrônica $\kappa_{e l}$ também é alta. Se esta for muito maior que a $\kappa_{p h}$ podemos, numa primeira aproximação, ignorar $\kappa_{p h}$ na Eq. (2.57). No entanto, devemos ter em mente que ao fazer esta aproximação estamos calculando um limite superior para $Z$, principalmente se $\kappa_{p h}$ não for muito menor que $\kappa_{e l}$.

Com esta aproximação temos

$$
Z \theta=\frac{G S^{2} \theta}{\kappa_{e l}}
$$


Usando os termos $K_{n}$, definidos na Eq. 2.47), podemos reescrever $G, S$ e $\kappa_{e l}$ :

$$
\begin{aligned}
G & =\frac{e^{2}}{h} K_{0} \\
S & =-\frac{1}{e \theta} \frac{K_{1}}{K_{0}} \\
\kappa_{e l} & =\frac{1}{h \theta}\left(K_{2}+\frac{K_{1}^{2}}{K_{0}}\right),
\end{aligned}
$$

e, consequentemente, obtemos

$$
Z \theta=\left(\frac{K_{2} K_{0}}{K_{1}^{2}}-1\right)^{-1}
$$

Atualmente, os dispositivos termoelétricos comerciais apresentam $Z \theta \approx 1$ [8, 9, 12. Em aplicações de refrigeração, isto significa uma eficiência em torno de um quarto da eficiência de sistemas convencionais, baseados em fluidos [8, 9]. Estima-se que seria necessário materiais com uma figura de mérito $Z \theta \geqslant 4$ para que dispositivos termoelétricos pudessem competir com esses sistemas convencionais [8, 9, 12]. No entanto, a partir de $Z \theta \sim 2$, algumas aplicações domésticas, como refrigeradores para processadores, se tornariam práticas [8].

\subsubsection{Implementação computacional}

A implementação computacional do cálculo das propriedades termoelétricas foi realizada sobre o código TRANSAMPA [14, 15], que, basicamente, calcula a transmitância para um dado sistema em um grid de energia com pontos regularmente espaçados. Os extremos do intervalo de energia e o número de pontos no grid são dados de entrada passados para o programa.

Inicialmente, implementamos o cálculo do coeficiente de Seebeck na expansão de 
Sommerfeld (Eq. 2.43), que como vimos, é dado por

$$
S=-\left.\frac{\pi^{2} k_{B}^{2}}{3 e} \bar{\theta} \frac{\partial \ln (T)}{\partial E}\right|_{E_{F}} .
$$

Logo, conhecida a transmitância $T(E)$, basta calcular sua derivada logarítmica.

Um método numérico para calcular a derivada é o de diferenças finitas, que é baseado na expansão local da função em uma série de Taylor,

$$
f(x+h)=f(x)+f^{\prime}(x) h+f^{\prime \prime}(x) \frac{h^{2}}{2}+f^{\prime \prime \prime}(x) \frac{h^{3}}{6}+\ldots
$$

de onde obtemos

$$
\begin{aligned}
f^{\prime}(x) & =\frac{f(x+h)-f(x)}{h}+\left[f^{\prime \prime}(x) \frac{h}{2}+\ldots\right] \\
& \approx \frac{f(x+h)-f(x)}{h} .
\end{aligned}
$$

O erro na estimativa da derivada, cujo termo majoritário é $f^{\prime \prime}(x) h / 2$, pode ser diminuído usando mais pontos para fazer a expansão. Somando

$$
f(x-h)=f(x)-f^{\prime}(x) h+f^{\prime \prime}(x) \frac{h^{2}}{2}-f^{\prime \prime \prime}(x) \frac{h^{3}}{6}+\ldots
$$

com 2.65 obtemos

$$
f^{\prime}(x)=\frac{f(x+h)-f(x-h)}{2 h}+\left[f^{\prime \prime \prime}(x) \frac{h^{2}}{3}+\ldots\right] .
$$

Na nossa implementação, usamos quatro pontos para calcular a derivada,

$$
f^{\prime}(x)=\frac{-f(x+2 h)+8 f(x+h)-8 f(x+2 h)+f(x-2 h)}{12 h}+\left[f^{(5)}(x) \frac{h^{4}}{30}+\ldots\right] .
$$

A derivada em (2.64) pode se calculada de duas formas distintas. Uma é computando 
$\ln (T(E))$ e depois tomando a derivada,

$$
\frac{\partial}{\partial E}[\ln (T(E))]
$$

E a outra é usando a regra da cadeia, o que resulta em

$$
\frac{1}{T(E)} \frac{\partial T(E)}{\partial E}
$$

Optamos pela segunda opção, pois a função logaritmo varia muito rapidamente se $T(E)<1$, que como veremos mais adiante é algo comum. Além disso, usando 2.72 adicionamos a informação de mais um ponto no cálculo da derivada, tornando seu valor mais preciso.

Na sequência, implementamos o código para calcular os termos $K_{n}$ (Eq. 2.47), dados por

$$
K_{n}=\int T(E)\left(-\frac{\partial f}{\partial E}\right)(E-\mu)^{n} d E
$$

pois $S, G, \kappa_{e l}$ e $Z \theta$ (Eqs. 2.61 2.63) são escritas em função destes termos. Neste caso, é preciso tomar cuidado com o termo

$$
\frac{\partial f}{\partial E}=-\frac{1}{k_{B} \theta} \frac{\exp (\epsilon)}{[\exp (\epsilon)+1]^{2}}
$$

onde

$$
\epsilon=\frac{E-\mu}{k_{B} \theta}
$$

Primeiro, devemos notar que se $k_{B} \theta$ for muito pequeno, o denominador de 2.74 explode (isto ocorre para $\theta \lesssim 70 \mathrm{~K}$ se $E-\mu=2 \mathrm{eV}$, por exemplo). Isto é resolvido 
usando a simetria

$$
\begin{aligned}
\frac{\partial f(\epsilon)}{\partial \epsilon} & =\frac{\partial f(-\epsilon)}{\partial \epsilon} \\
& =\frac{\exp (-\epsilon)}{[\exp (-\epsilon)+1]^{2}}
\end{aligned}
$$

Outro ponto muito importante é o espaçamento do grid usado no cálculo. A forma da função $\partial f / \partial E$ é a de um pico em torno de $\mu$ com altura $1 / 4 k_{B} \theta$ e uma largura de aproximadamente $7 k_{B} \theta$. Portanto, o grid deve ter um espaçamento menor que $3.5 k_{B} \theta$. Caso contrário, $\partial f / \partial E$ estará mal descrita, o que pode arruinar o integrando em (2.73). Contudo, há uma forma de mitigar este problema. Se o valor de $\mu$ em 2.75 coincidir com um ponto de energia do grid, teremos

$$
\left.\frac{\partial f}{\partial E}\right|_{E=\mu}=-\frac{1}{4 k_{B} \theta},
$$

o que, de certa forma, corresponde a aproximar de $\partial f / \partial E$ por uma função delta numérica. Em nossa implementação, os valores de $\mu$ sempre coincidem com os pontos do grid de energia.

O método numérico escolhido para computar a integral 2.73 foi o do trapezoide, que é um método robusto e simples e é um dos mais indicados para calcular integrandos de funções arbitrárias, das quais não se tem conhecimento a priori (no nosso caso, $T(E))$ [69].

Com estas implementações, dispomos de todas as ferramentas necessárias para calcular as propriedades termoelétricas de sistemas nanoscópicos na formulação de Landauer. 


\section{Capítulo 3}

\section{Nanofita zigzag}

Vamos agora apresentar os resultados obtidos no estudo das propriedades termoelétricas de nanofitas de grafeno zigzag pristinas (puras, sem defeitos) e com impurezas substitucionais de Boro e Nitrogênio nas bordas.

Os cálculos de estrutura eletrônica aqui apresentados foram realizados com o pacote SIESTA [70, 71], baseado na Teoria do Funcional da Densidade (veja o apêndice A e que usa uma base de funções estritamente localizadas, pseudopotenciais de Troullier-Martins e condições periódicas de contorno. Os cálculos foram realizados para fitas de largura 1,56 nm (o que corresponde a sete hexágonos), para os quais usamos uma supercélula com 14 repetições de uma célula unitária (correspondendo a um comprimento de $3,5 \mathrm{~nm}$ ), incluindo uma camada de vácuo de $16 \AA$ nas direções perpendiculares ao eixo da fita, de forma que não houvesse interações entre as imagens das fitas. Em todos os casos as geometrias foram relaxadas até que a força residual nos átomos fosse menor que $0,01 \mathrm{eV} / \AA$.

Já os cálculos das propriedades de transporte foram realizados com o código TRANSAMPA [14, 15], que é baseado em funções de Green fora do equilíbrio e DFT. Para os eletrodos, usamos uma supercélula com três repetições de uma célula unitária. Na figura 3.1 apresentamos uma ilustração da geometria dos problemas de transporte calculados neste trabalho. Na ilustração podemos ver a região central 


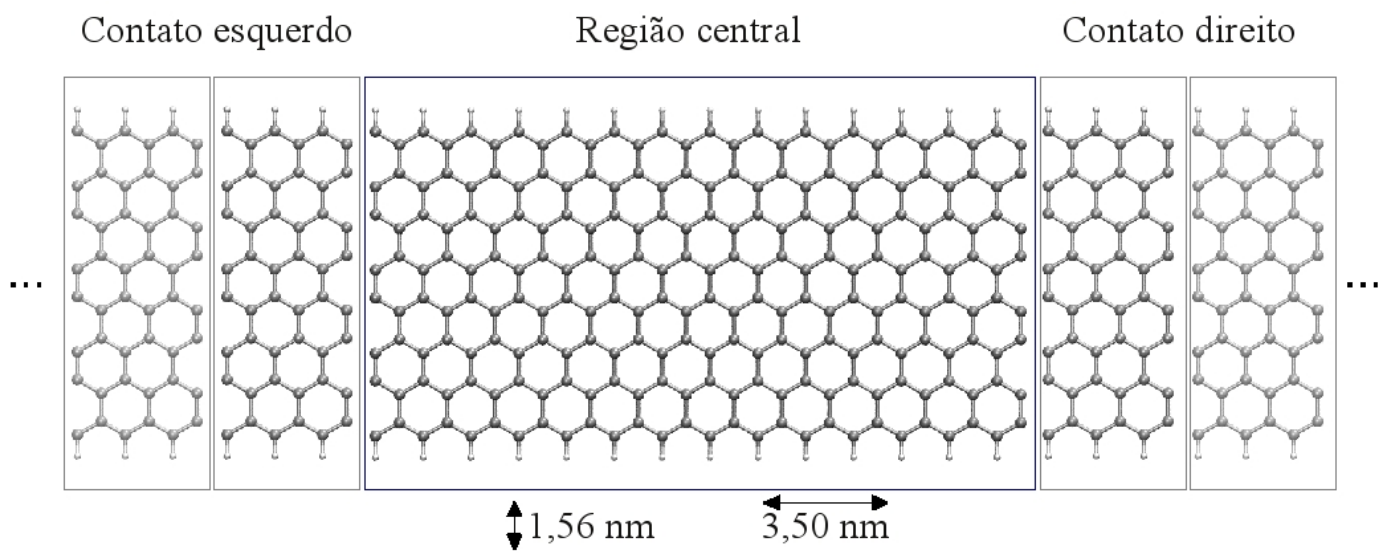

Figura 3.1: Ilustração da geometria dos contatos e da região central nos problemas estudados.

(no caso, uma nanofita pristina) e as geometrias dos blocos de eletrodo.

Inicialmente, vamos estudar impurezas substitucionais de Boro e Nitrogênio na borda de nanofitas na configuração não-magnética (isto é, sem levar em conta polarização de spin). Escolhemos este sítio (a borda) por ser o que apresenta a menor energia de formação para este tipo de defeito, como mostrado por Martins et al. [72].

As configurações eletrônicas da camada de valência do Boro, Carbono e Nitrogênio são $2 s^{2} 2 p^{1}, 2 s^{2} 2 p^{2}$ e $2 s^{2} 2 p^{3}$, respectivamente. Portanto, como o Boro possui um elétron a menos em relação ao Carbono, é de se esperar que o B injete um buraco no sistema, enquanto que o Nitrogênio, por possuir um elétron a mais, injete um elétron.

$\mathrm{Na}$ parte superior da figura 3.2 podemos ver as geometrias de uma nanofita pristina e com impurezas de B e $\mathrm{N}$ na borda (figs. $3.2 \mathrm{a}-\mathrm{c}$ ). Como se pode ver na figura, para o caso da impureza de Boro, aparece um estado desocupado em torno de $0.65 \mathrm{eV}$ acima do nível de Fermi (indicada na cor verde na Fig. 3.2 $)$, que é devido ao buraco introduzido pelo B, e que abre um gap de energia entre os estados de Carbono. Já para o N, temos um estado ocupado em torno de 0.6 eV abaixo do nível de Fermi (em cor azul na Fig. 3.2f), devido ao elétron injetado e que também abre um gap. As 


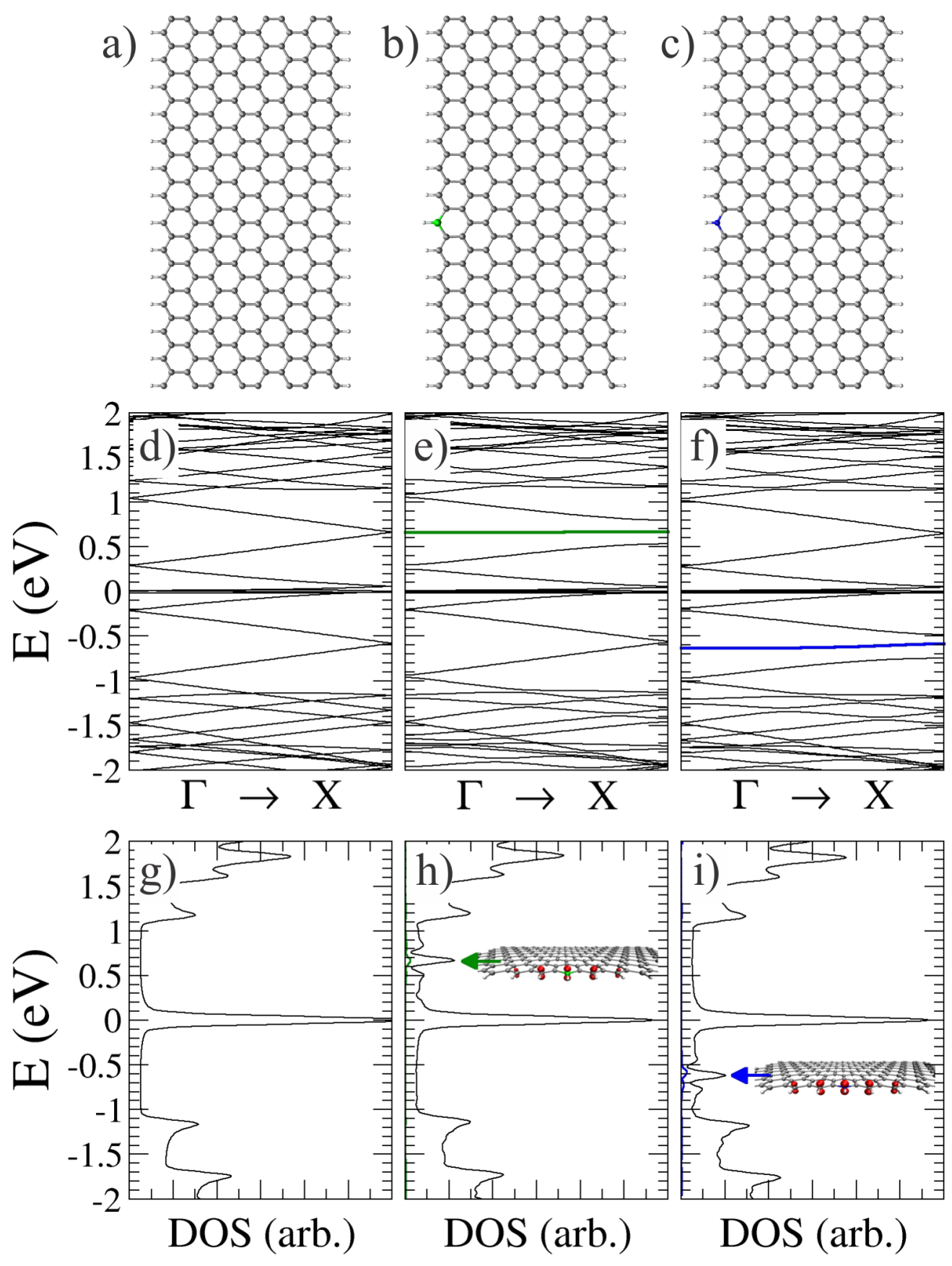

Figura 3.2: Geometrias (a-c), estrutura de bandas (d-f) e densidade de estados (g-i) de uma nanofita de grafeno $(7,0)$ pristina (coluna da esquerda), com uma impureza de Boro na borda (no meio) e com uma impureza de Nitrogênio (na direita). As imagens nas DOS das impurezas de $\mathrm{B}$ e $\mathrm{N}$ mostram a LDOS dos estados das impurezas correspondentes (isosuperfície com $0.012 \mathrm{e} / \AA$ ). 
respectivas densidades de estados (Density Of States - DOS), que podem ser vistas na parte inferior da figura 3.2, mostram, como esperado, o aparecimento de picos na DOS correspondentes aos estados localizados das impurezas, indicados com setas nas figs. 3.2 h-i. As imagens inseridas nos gráficos da DOS das impurezas, mostram a densidade local de estados (Local Density Of States - LDOS) correspondentes a janela de energia em torno dos estados das impurezas, onde se pode ver que estes estados são localizados na borda, em torno das impurezas.

A transmitância obtida para uma nanofita de grafeno $(7,0)$ pura pode ser vista na figura 3.3 junto com sua densidade de estados projetada. Como esperado para um sistema pristino, a transmitância apresenta um formato de escada, aumentando ou diminuindo conforme o número de canais de condução (estados) disponíveis para cada energia. Além disso, podemos notar as singularidades de Van Hove na DOS (PDOS total), típicas de sistemas unidimensionais e um pico da DOS na energia do nível de Fermi, devido, principalmente, aos estados de borda (cuja PDOS está plotada em vermelho na Fig. 3.3b). Assim, o pico na transmitância na energia de Fermi é causado, majoritariamente, pelos estados de borda da fita.

Quando a impureza de Boro é introduzida, como vimos anteriormente, temos o surgimento de um estado localizado com energia em torno de $0,65 \mathrm{eV}$. Correspondentemente, temos um pico da PDOS do sistema nessa energia, como pode ser visto na figura 3.4b. Na transmitância, que está plotada na Fig. 3.4a, observamos uma queda na probabilidade de transmissão eletrônica a praticamente zero em torno da energia desse estado. Esta queda ocorre devido ao espalhamento causado por este estado desocupado do Boro, que resulta interferência destrutiva.

Da mesma forma, no caso da impureza de Nitrogênio também temos um estado localizado. Portanto, igualmente ao caso do Boro, na região em torno da energia deste estado introduzido pelo $\mathrm{N}$, em torno de $-0.6 \mathrm{eV}$, onde há o pico na PDOS, como visto no gráfico da PDOS na Fig. 3.5b, a transmitância do sistema cai a praticamente zero (Fig. 3.5 ). Novamente, esta queda na transmitância é devida ao 

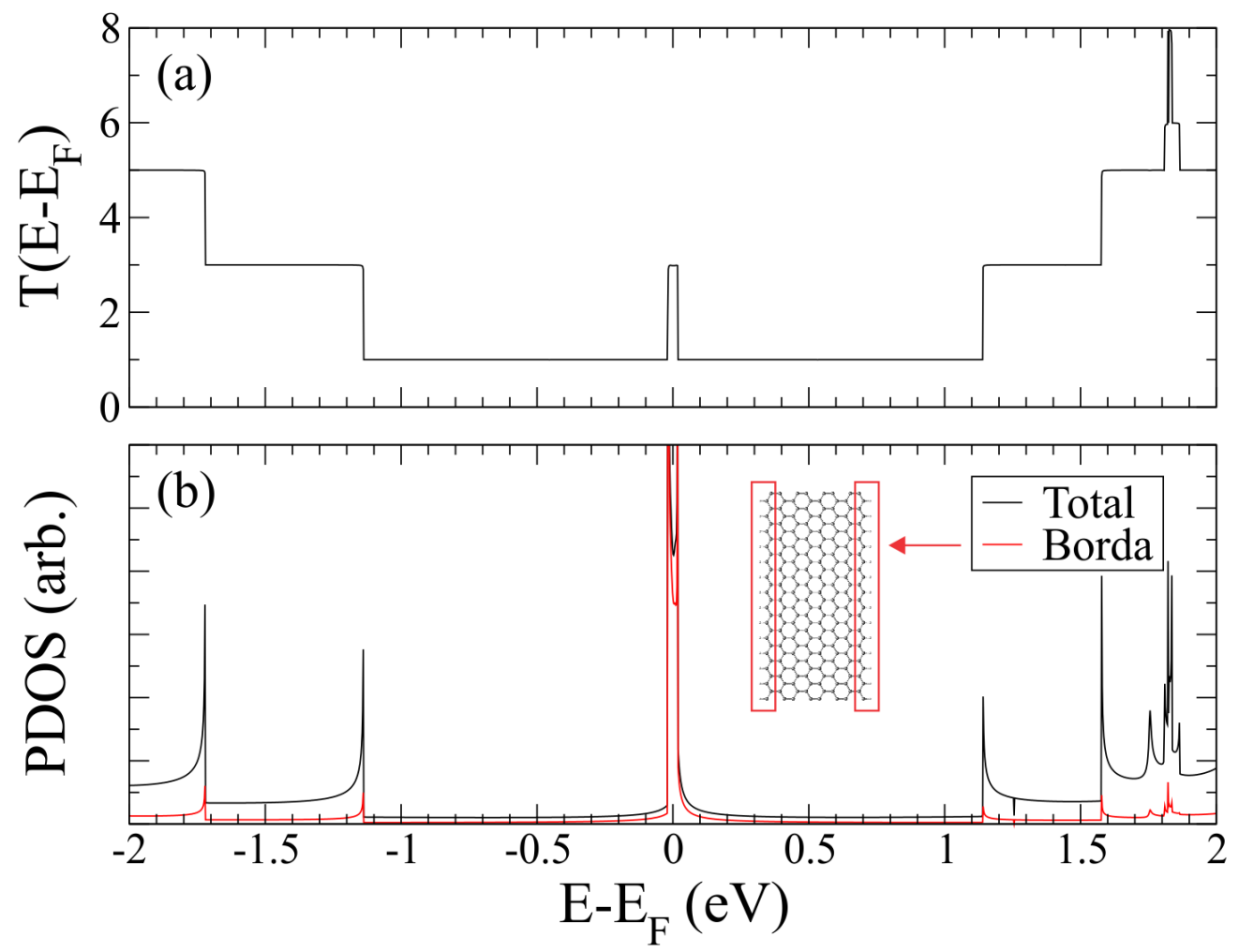

Figura 3.3: Transmitância (a) e densidade de estados (b) de uma nanofita $(7,0)$ pristina em função da energia. Na imagem em (b) mostramos quais átomos definimos como sendo de borda (dentro dos retângulos em vermelho).

espalhamento causado pelo estado da impureza.

Vemos assim, que as duas impurezas introduzem estados localizados que causam espalhamento, fazendo que a transmitância do sistema caia a zero na região de energia destes níveis.

Na figura 3.6 apresentamos a condutância, $G$, o coeficiente Seebeck, $S$, a condutância térmica eletrônica, $\kappa_{e l}$ e a figura de mérito adimensional, $Z \theta$, obtidas através das eqs. 2.61 2.63 para várias temperaturas médias dos contatos. Como se pode ver na figura, a condutância eletrônica, $G$, apresenta um perfil muito semelhante ao da transmitância (Fig. 3.3), no qual a temperatura causa um efeito de alargamento e suavização da curva, como é esperado da Eq. 2.14.

$$
G=\frac{2 e^{2}}{h} \int T(E)\left(-\frac{\partial f}{\partial E}\right) d E
$$



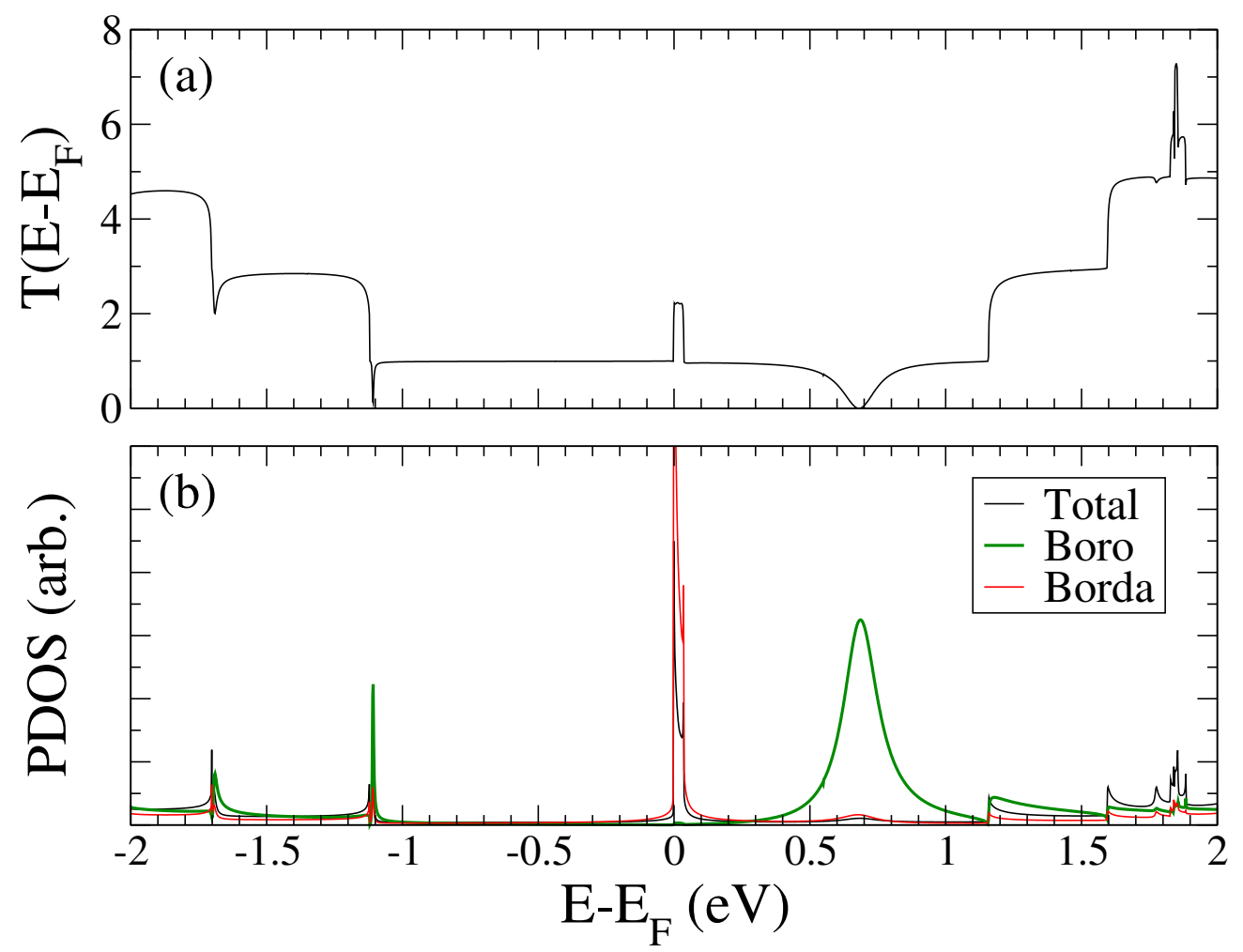

Figura 3.4: Transmitância (a) e densidade de estados projetada normalizada (b) em todos os átomos (total), na borda e no Boro para uma nanofita $(7,0)$ com uma impureza de Boro na borda.

onde podemos notar que a condutância $G$ é dada por uma convolução da transmitância numa janela de energias da ordem de $k_{B} \theta$.

O coeficiente de Seebeck apresenta picos nas energias onde canais de condução são adicionados ou removidos, atingindo valores máximos de $65 \mu V / K$. Da mesma forma, $Z \theta$ também apresenta picos nessas energias, alcançando valores máximos em torno de 0,18. Logo, conforme o visto na seção 2.3.4 nanofitas de grafeno zigzag pristinas não são bons materiais para aplicações termoelétricas (geradores ou refrigeradores/aquecedores) porque apresentam $Z \theta$ baixo.

Na figura 3.7 podemos ver os resultados obtidos para as condutâncias eletrônica e térmica eletrônica, $G$ e $\kappa_{e l}$, e para as propriedades termoelétricas: o coeficiente Seebeck e a figura de mérito adimensional para uma nanofita $(7,0)$ com um impureza de Boro na borda. Como vimos anteriormente, o efeito da impureza de Boro consiste, 

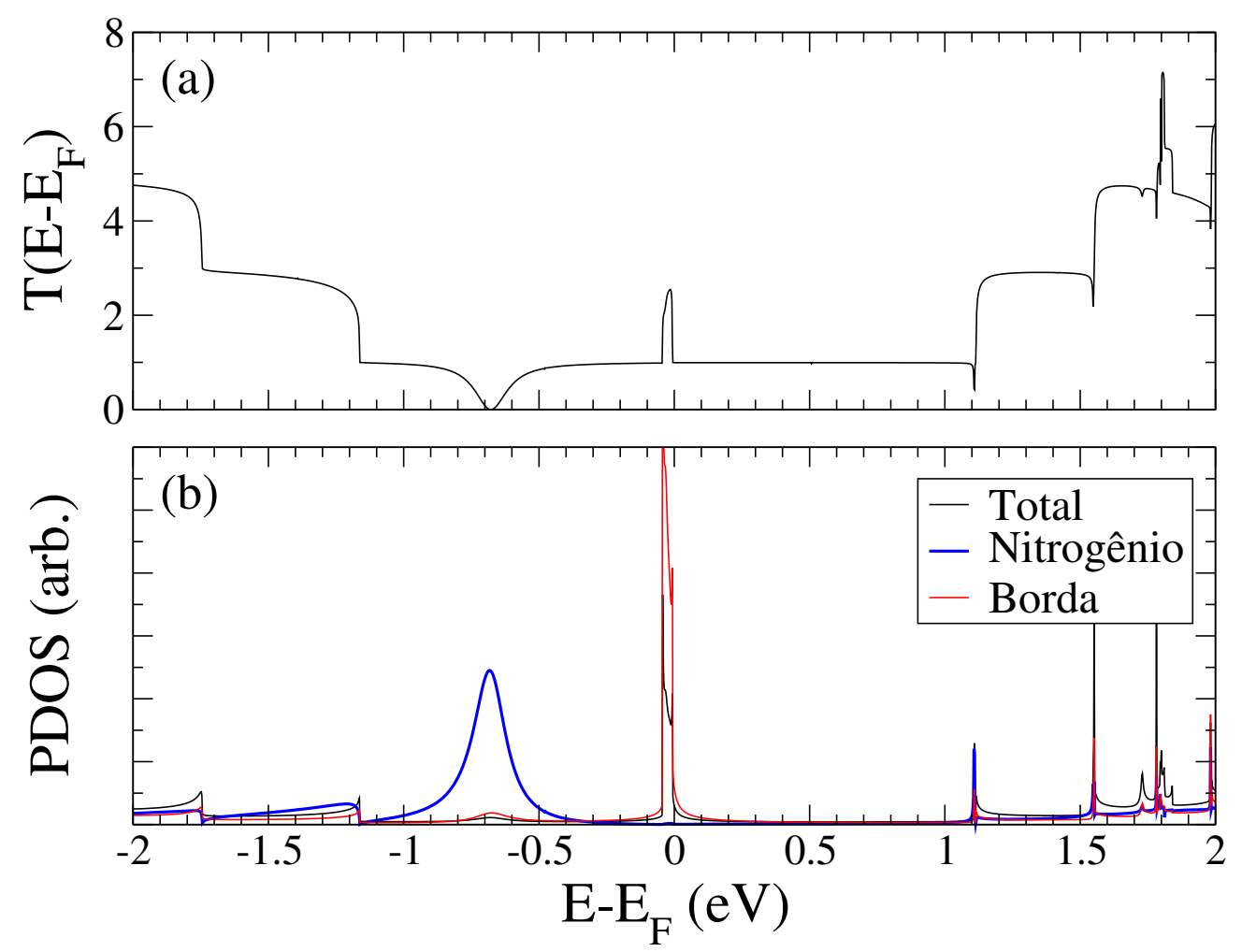

Figura 3.5: Transmitância (a) e densidade de estados projetada normalizada (b) em todos os átomos (total), na borda e no Nitrogênio para uma nanofita $(7,0)$ com uma impureza de Nitrogênio na borda.

majoritariamente, em uma queda da transmitância na região de energia associada ao nível do buraco (Fig. 3.4 a). Consequentemente, há uma queda nas condutâncias eletrônica $G$ e térmica eletrônica $\kappa_{e l}$, o que se reflete em dois picos no coeficiente Seebeck $S$ e na figura de mérito adimensional $Z \theta$ adjacentes (em energia) a esse nível.

É interessante notar o aumento significativo do coeficiente Seebeck e da figura de mérito adimensional na região de energia associada ao nível da impureza. $S$ atinge valores de aproximadamente $150 \mu \mathrm{V} / \mathrm{K}$ e $90 \mu \mathrm{V} / \mathrm{K}$ para $\theta=10 \mathrm{~K}$ e $\theta=$ $300 K$, respectivamente (lembre que para a fita pristina $S_{\max } \approx 65 \mu V / K$ ). Portanto, (lembre que $Z \theta=S^{2} G / \kappa_{e l}$ ), temos um aumento na figura de mérito $Z \theta$, com valores máximos de $\sim 0,7$ e $\sim 0.3$ para $\theta=10 K$ e $\theta=300 K$, respectivamente.

No caso da impureza de Nitrogênio observamos o mesmo tipo de comportamento 

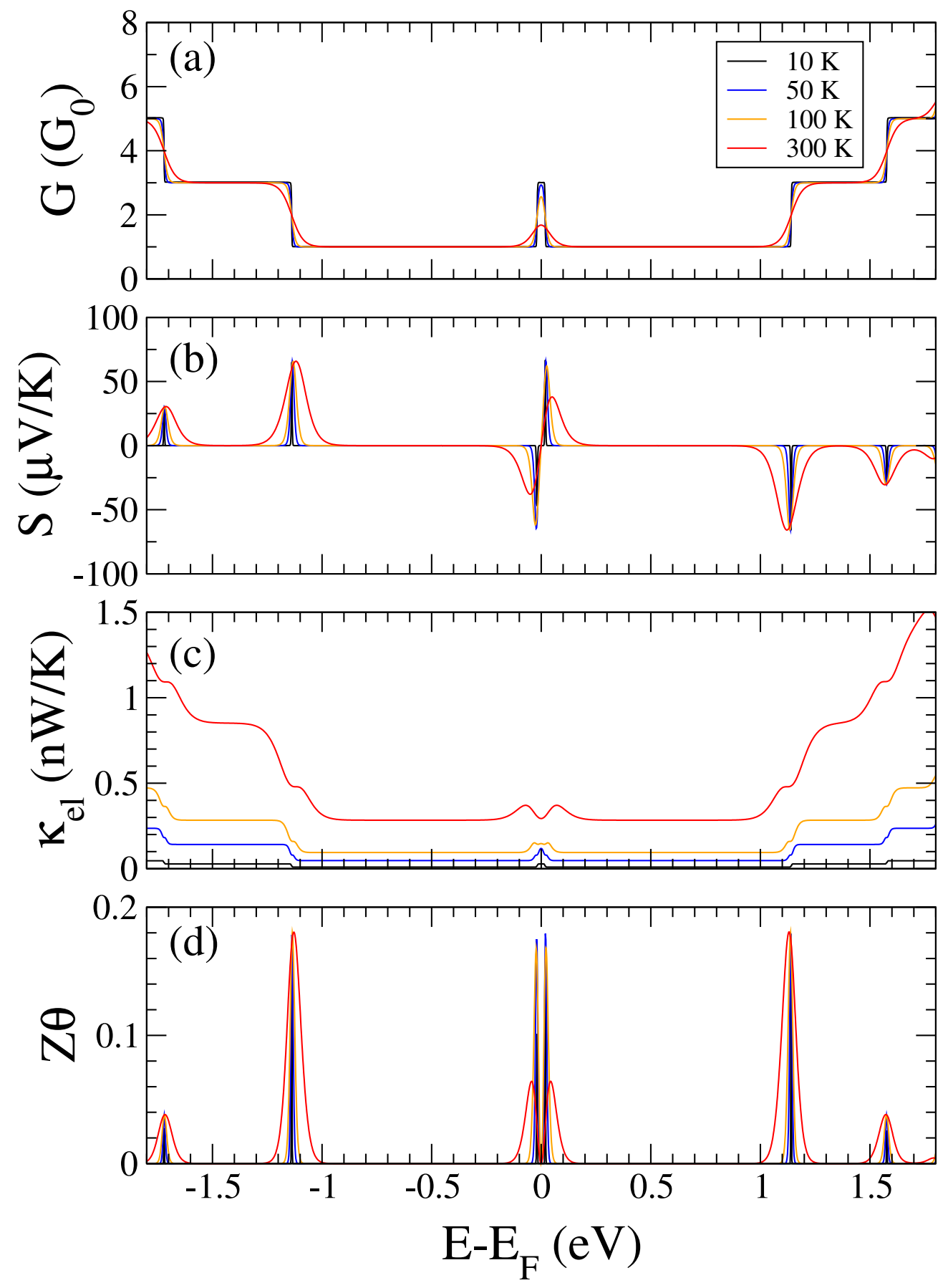

Figura 3.6: Condutância, $G$, coeficiente Seebeck, $S$, condutância térmica eletrônica, $\kappa_{e l}$, e figura de mérito adimensional, $Z \theta$ de uma nanofita $(7,0)$ pristina para várias temperaturas. 

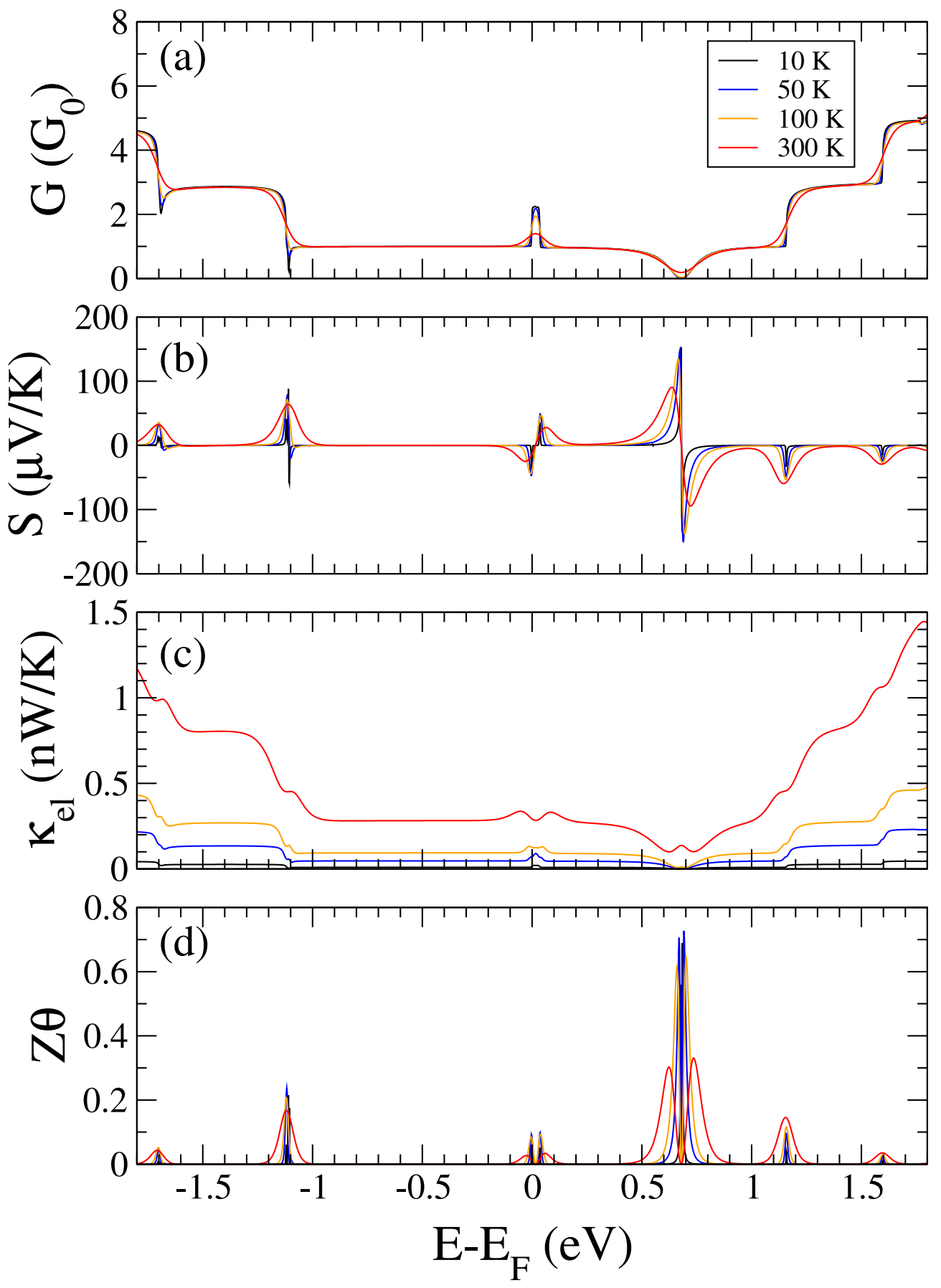

Figura 3.7: Condutância, $G$, coeficiente Seebeck, $S$, condutância térmica eletrônica, $\kappa_{e l}$, e figura de mérito adimensional, $Z \theta$ de uma nanofita $(7,0)$ com uma impureza de Boro na borda para várias temperaturas. 

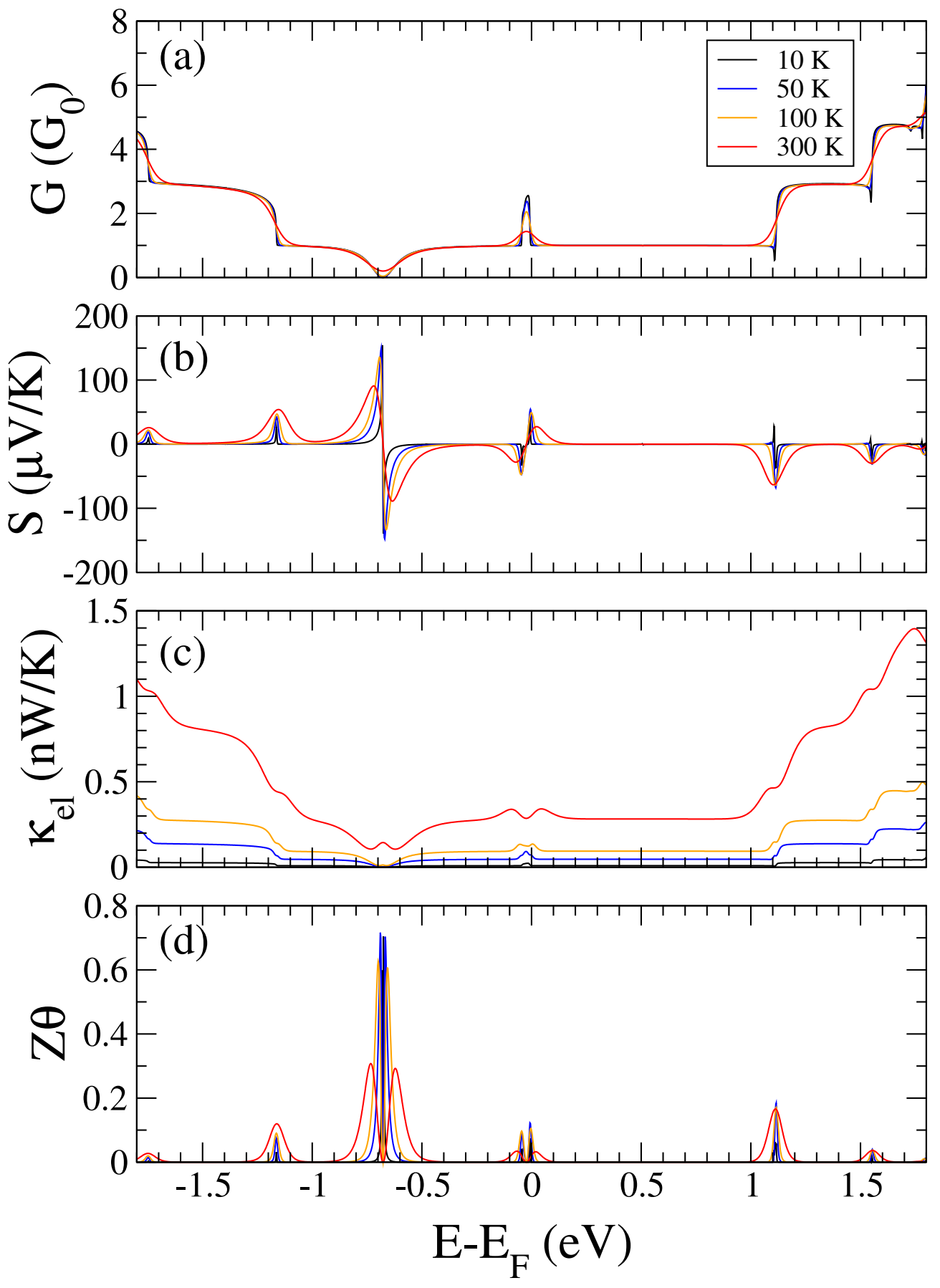

Figura 3.8: Condutância, $G$, coeficiente Seebeck, $S$, condutância térmica eletrônica, $\kappa_{e l}$, e figura de mérito adimensional, $Z \theta$ de uma nanofita $(7,0)$ com uma impureza de Nitrogênio na borda para várias temperaturas. 
(veja a figura 3.5 que para a impureza de Boro, mas nas energia do nível do elétron injetado pelo $\mathrm{N}$ (em torno de $-0.7 \mathrm{eV}$ ), obviamente (inclusive com os mesmos valores). Explicitando, temos as quedas nas condutâncias $G$ e $\kappa_{e l}$ na energia do nível da impureza, que se refletem nos picos no coeficiente Seebeck $S$ e na figura de mérito adimensional $Z \theta$.

Quando consideramos o estado ferromagnético da nanofita, temos a estrutura de bandas e as densidades de estados mostradas na figura 3.9 para as fitas pristina, e com impurezas de Boro e Nitrogênio. Nesta configuração, como se pode ver nas estruturas de bandas e nas DOS, há um separação em energia dos estados de spins $\alpha$ e $\beta$ das impurezas. Logo, da mesma forma que no caso sem polarização de spin (Fig. 3.2), as impurezas introduzem estados localizados no sistema. No entanto, agora, os níveis (spin $\alpha$ e $\beta$ ) de cada impureza apresentam uma separação em energia, o que se traduz como dois picos na DOS, um para o spin $\alpha$ (indicado pelas setas cheias na Fig. 3.9) do nível da impureza, e um para o $\beta$ (indicado pelas setas vazadas na Fig. 3.9). Os estados da impureza de Boro se localizam em torno de 0,56 eV ( $\operatorname{spin} \alpha)$ e $0,73 \mathrm{eV}(\operatorname{spin} \beta)$. Já os do Nitrogênio, em torno de $-0,57 \mathrm{eV}(\operatorname{spin} \alpha)$ e $-0,75 \mathrm{eV}$ $(\operatorname{spin} \beta)$.

A transmitância obtida para uma nanofita zigzag pristina na configuração ferromagnética pode ser vista no gráfico do painel superior da figura 3.10 , onde verificamos que há uma anisotropia nas transmitâncias para os canais de spin $\alpha$ e de spin $\beta$ em determinados intervalos de energia. Estes, correspondem as regiões onde as densidades de estados dos spins $\alpha$ e $\beta$ diferem, como pode ser visto no painel inferior da figura 3.10 .

Na figura 3.11 plotamos a transmitância e a densidade de estados projetada para uma nanofita $(7,0)$ com uma impureza de Boro na borda, na configuração ferromagnética. Como podemos observar no gráfico, a transmitância do sistema (Fig. 3.11a) apresenta anisotropia de spin, da mesma forma que no caso da nanofita pristina, e, semelhantemente ao cálculo sem polarização (Fig. 3.4a), a transmitância 

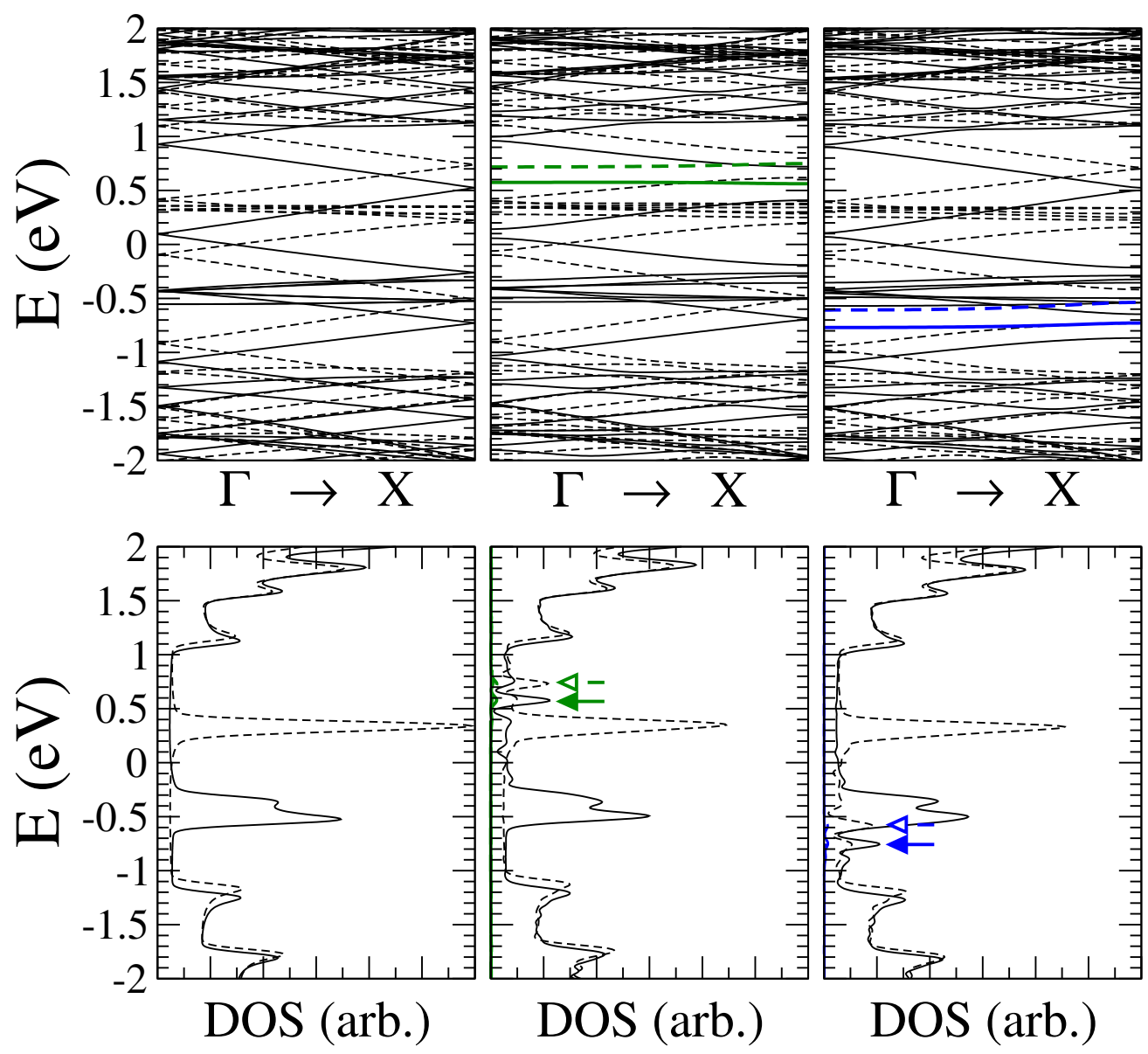

Figura 3.9: Estrutura de bandas (parte superior) e densidade de estados (parte inferior) de uma nanofita de grafeno $(7,0)$ pristina na configuração ferromagnética (na esquerda), com uma impureza de Boro na borda (no meio) e com uma impureza de Nitrogênio (na direita). Linhas contínuas são referentes ao spin $\alpha$ e linhas tracejadas ao spin $\beta$. As setas nas DOS indicam os níveis das impurezas. 

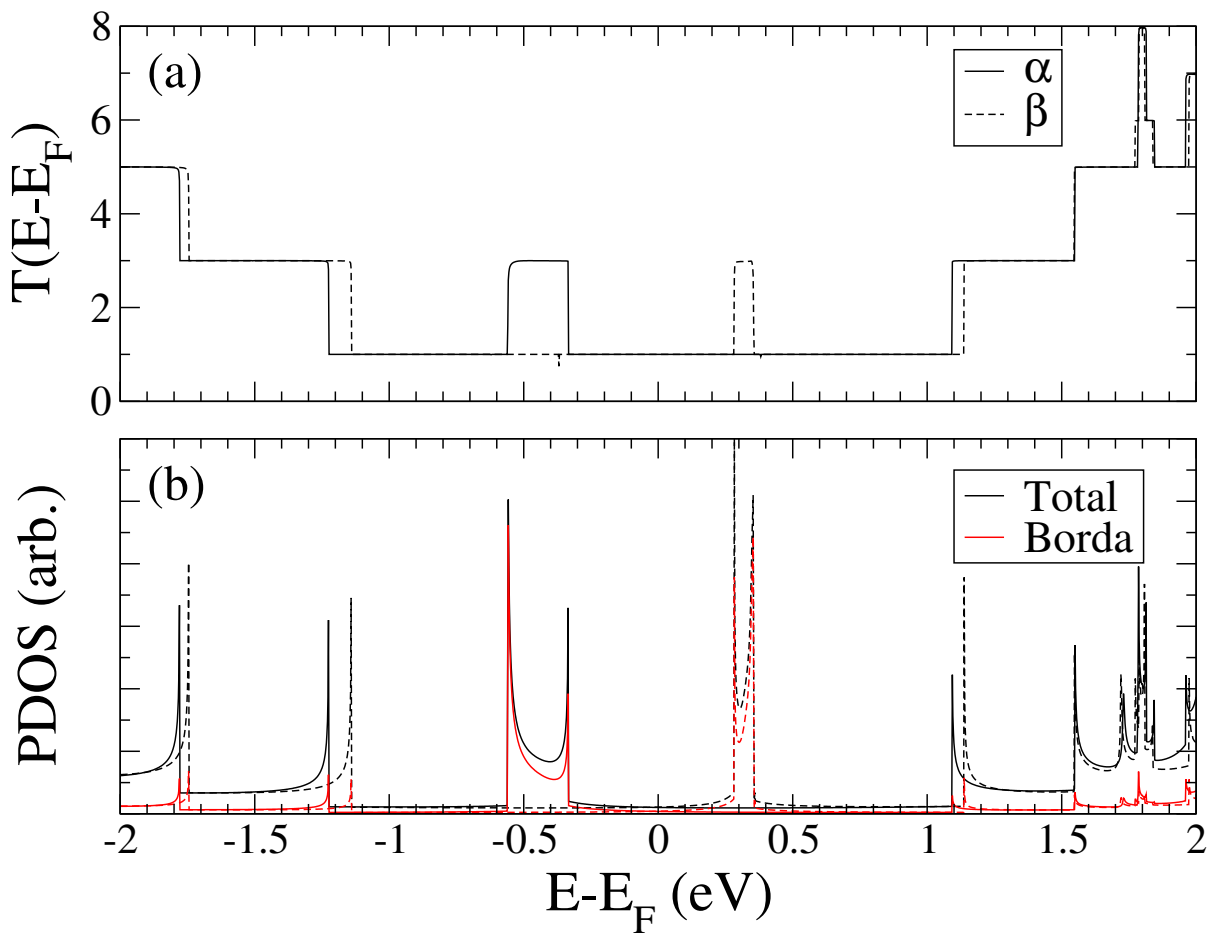

Figura 3.10: Transmitância (a) e densidades de estados projetada (b) em todos os átomos (total) e nos átomos de borda para uma nanofita $(7,0)$ pristina na configuração ferromagnética.

se torna praticamente nula nas energias dos níveis da impureza de B. Da mesma forma que nos casos anteriores, isto se deve ao espalhamento causado pelos estados localizados do Boro.

Note que nas energias dos estados da impureza temos uma transmitância nula para um spin, e próxima de $1 G_{0}$ para o spin oposto. Por exemplo, na região de energia associada ao estado de spin $\alpha$ do $\mathrm{B}$, em torno de $\sim 0,6 \mathrm{eV}$, a transmitância de elétrons com spin $\alpha$ é praticamente nula, devido ao espalhamento por esse estado, enquanto que a transmitância de elétrons $\operatorname{com} \operatorname{spin} \beta$ é $\sim 1$. Já na região de energia do nível de spin $\beta$ da impureza de $\mathrm{B}$, em torno de $\sim 0,75 \mathrm{eV}$, temos o contrário: a transmitância para elétrons de spin $\alpha$ é $\sim 1$ e, para elétrons de spin $\beta$, nula. Logo, nessas energias apenas elétrons de um determinado spin são transmitidos, o que tornaria este sistema em um filtro de spin perfeito.

Já para a impureza de Nitrogênio numa nanofita na configuração ferromagnética, 

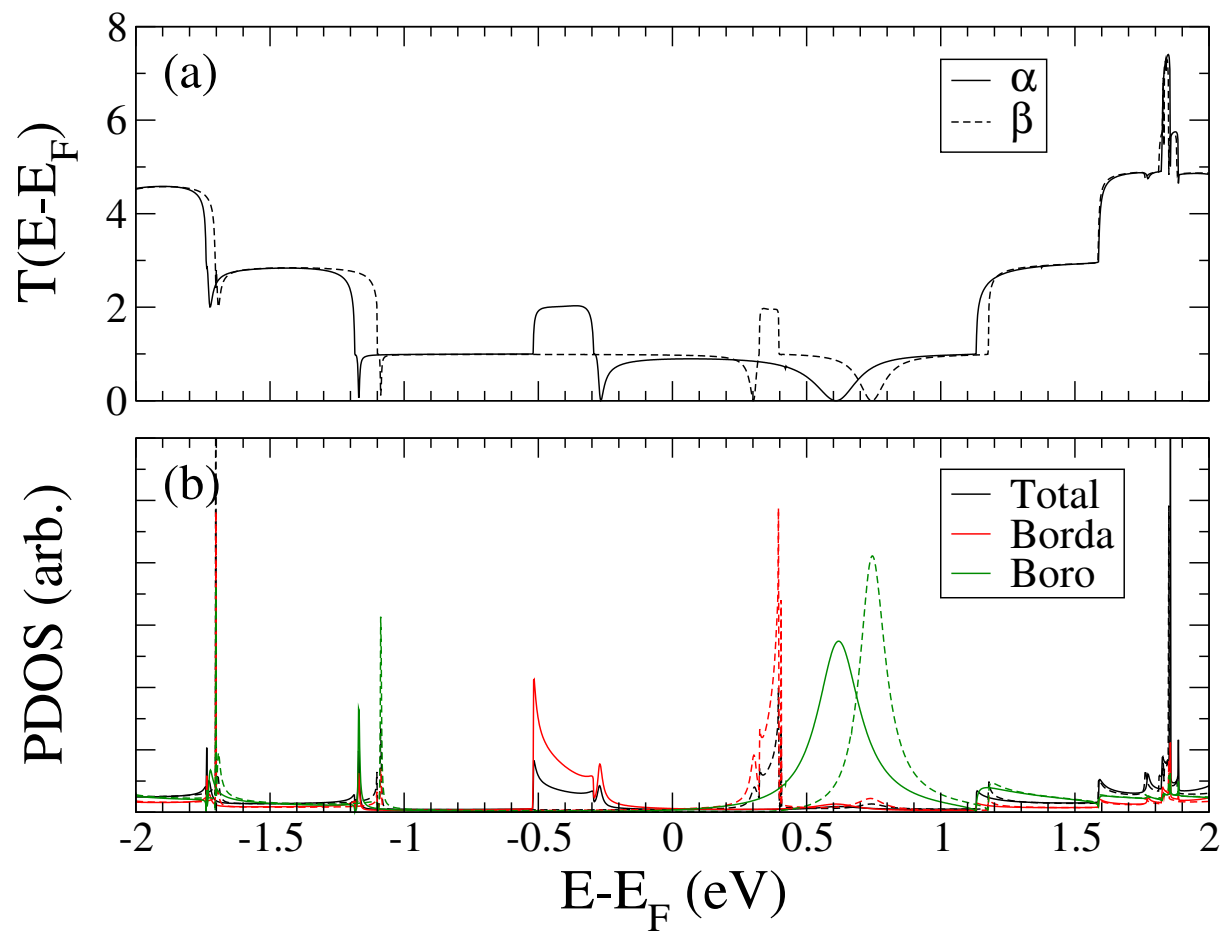

Figura 3.11: Transmitância (a) e densidades de estados projetada normalizada (b) em todos os átomos (total), nos átomos de borda e no Boro, para uma nanofita $(7,0)$ na configuração ferromagnética com uma impureza de Boro na borda.

temos a transmitância e a densidades de estados mostradas na figura 3.12. Como podemos ver, este sistema apresenta as mesmas características do caso anterior (Boro), com a diferença, é claro, das energias onde estão localizados os estados da impureza e as consequentes quedas na transmitância devido ao espalhamento causados por estes estados. Note, também, que a nanofita com a impureza de N permite a transmissão de elétrons em apenas um determinado canal de spin nas energias associadas aos níveis da impureza.

A seguir, apresentamos a condutâncias eletrônica, $G$, a condutância térmica eletrônica, $\kappa_{e l}$, o coeficiente Seebeck, $S$, e a figura de mérito, $Z \theta$, para nanofita $(7,0)$ na configuração ferromagnética pristina, e com impurezas de Boro e Nitrogênio, para várias temperaturas médias entre os contatos.

Os resultados obtidos para a nanofita pristina podem ser vistos na figura 3.13 . Como esperado, o gráfico da condutância $G$ segue o perfil da curva de transmitância 

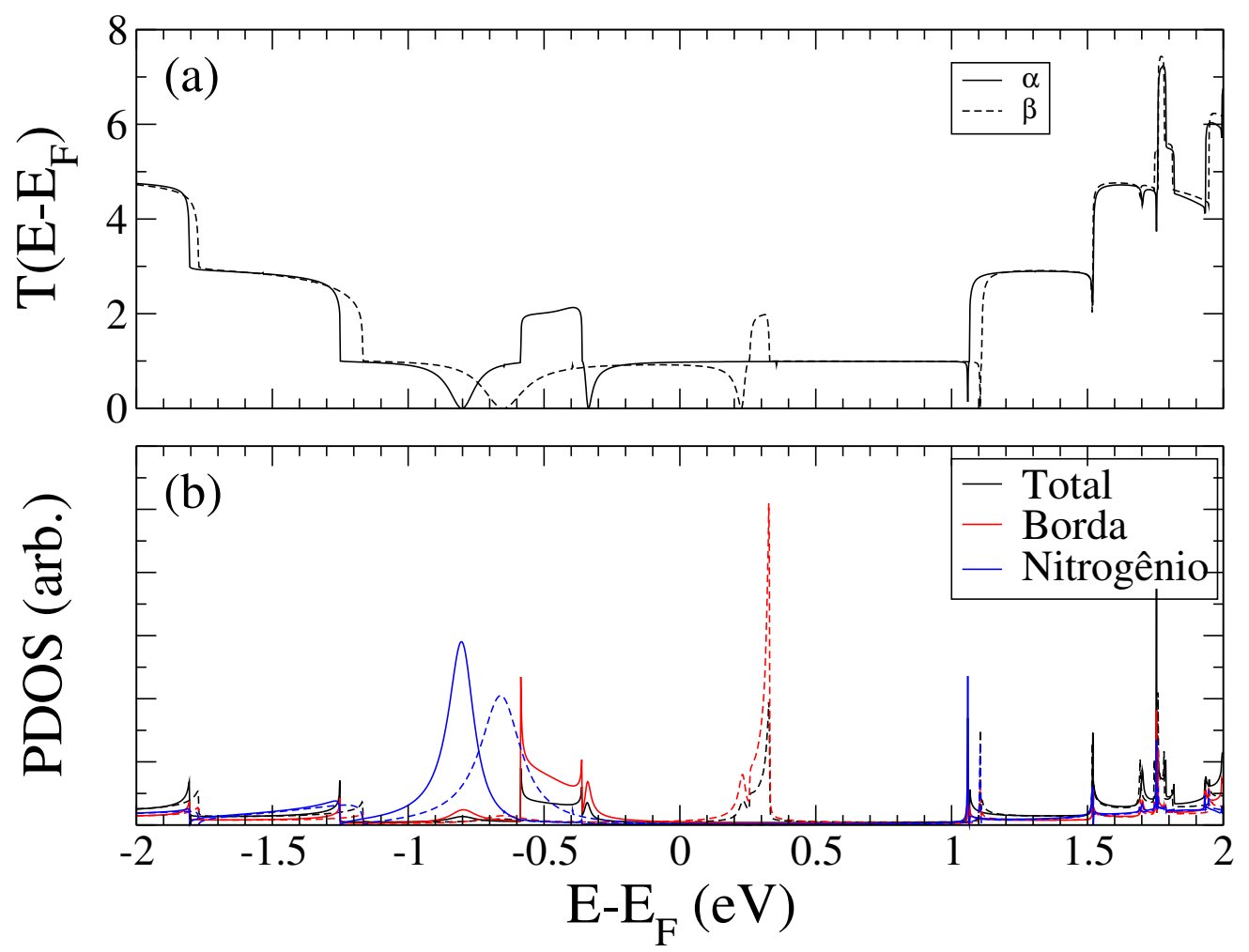

Figura 3.12: Transmitância (a) e densidades de estados projetada normalizada (b) em todos os átomos (total), nos átomos de borda e no Nitrogênio, para uma nanofita $(7,0)$ na configuração ferromagnética com uma impureza de Nitrogênio na borda.

(Fig. 3.10a) com o alargamento e suavização correspondentes devido à temperatura. No gráfico do coeficiente Seebeck (Fig. 3.13b) podemos perceber um fato muito interessante: a anisotropria de spin em $G$ é refletida na posição dos picos em $S$ para cada spin. Por exemplo, para energias em torno de $-0,55 \mathrm{eV}$ e $-0,32 \mathrm{eV}$ temos dois picos no coeficiente de Seebeck para o spin $\alpha$, com $S \approx \pm 65 \mu V / K$, enquanto que $S$ para o spin $\beta$ é nulo. Já para energias em torno de $0,27 \mathrm{eV}$ e $0,35 \mathrm{eV}, S$ é nulo para o spin $\alpha$ e apresenta um pico para o spin $\beta$. Desta forma, nanofitas de grafeno zigzag pristinas podem ser usadas como dispositivos de caloritrônica de spin [73], gerando uma corrente com spin determinado a partir de um gradiente de temperatura aplicado entre os eletrodos.

No gráfico para a figura de mérito $Z \theta$ (Fig. 3.13d) podemos ver que os picos também são separados por spin, como esperado dada a assimetria de spin na 
transmitância, e que $Z \theta$ atinge valores máximos de aproximadamente 0,18 , semelhantemente ao caso da nanofita na configuração não-magnética (Fig. 3.6.

Os resultados obtidos quando um impureza de Boro é introduzida em uma nanofita na configuração ferromagnética estão apresentados na figura 3.14. Como foi discutido anteriormente, os estados da impureza de B causam espalhamento no transporte nas suas respectivas energias e canais de spin, ocasionando uma queda correspondente (em torno dessas energias e para o respectivo spin) nas condutâncias eletrônica, $G$ (Fig. 3.14a) , e térmica eletrônica, $\kappa_{e l}$ (Fig. 3.14 ). Note também o aumento significativo no coeficiente de Seebeck (Fig. 3.14 b) em torno dos níveis do Boro, que atingem valores de $97(80) \mu V / K$ e $-108(-80) \mu V / K$ para $\theta=300 K$ para o spin $\alpha(\beta)$, e $150(-155) \mu V / K$ e $-150(-43) \mu V / K$ para $\theta=10 K$ para o spin $\alpha(\beta)$. Da mesma forma, a figura de mérito $Z \theta$ (Fig. 3.14 $\mathrm{d}$ ) também apresenta um aumento, com máximos de $\sim 0,7$ e $\sim 0,4$ para temperaturas médias entre os contatos de $10 \mathrm{~K}$ e $300 \mathrm{~K}$, respectivamente (a nanofita pristina apresenta $Z \theta$ máximo de $\sim 0,18)$.

Para o caso de uma impureza de Nitrogênio em uma nanofita na configuração ferromagnética temos resultados semelhantes aos da impureza de Boro, como pode ser visto na figura 3.15. Os estados da impureza, que agora se localizam abaixo do nível de Fermi (Fig. 3.9), causam espalhamento no transporte, que se reflete em uma queda nas condutâncias eletrônica (Fig. 3.15a) e térmica eletrônica (Fig. 3.15 ) em torno das energias desses níveis. O coeficiente Seebeck (Fig. 3.15b) e a figura de mérito (Fig. 3.15d) apresentam picos em torno das energias dos níveis da impureza com valores máximos significativamente mais altos que no caso de uma nanofita pristina. 

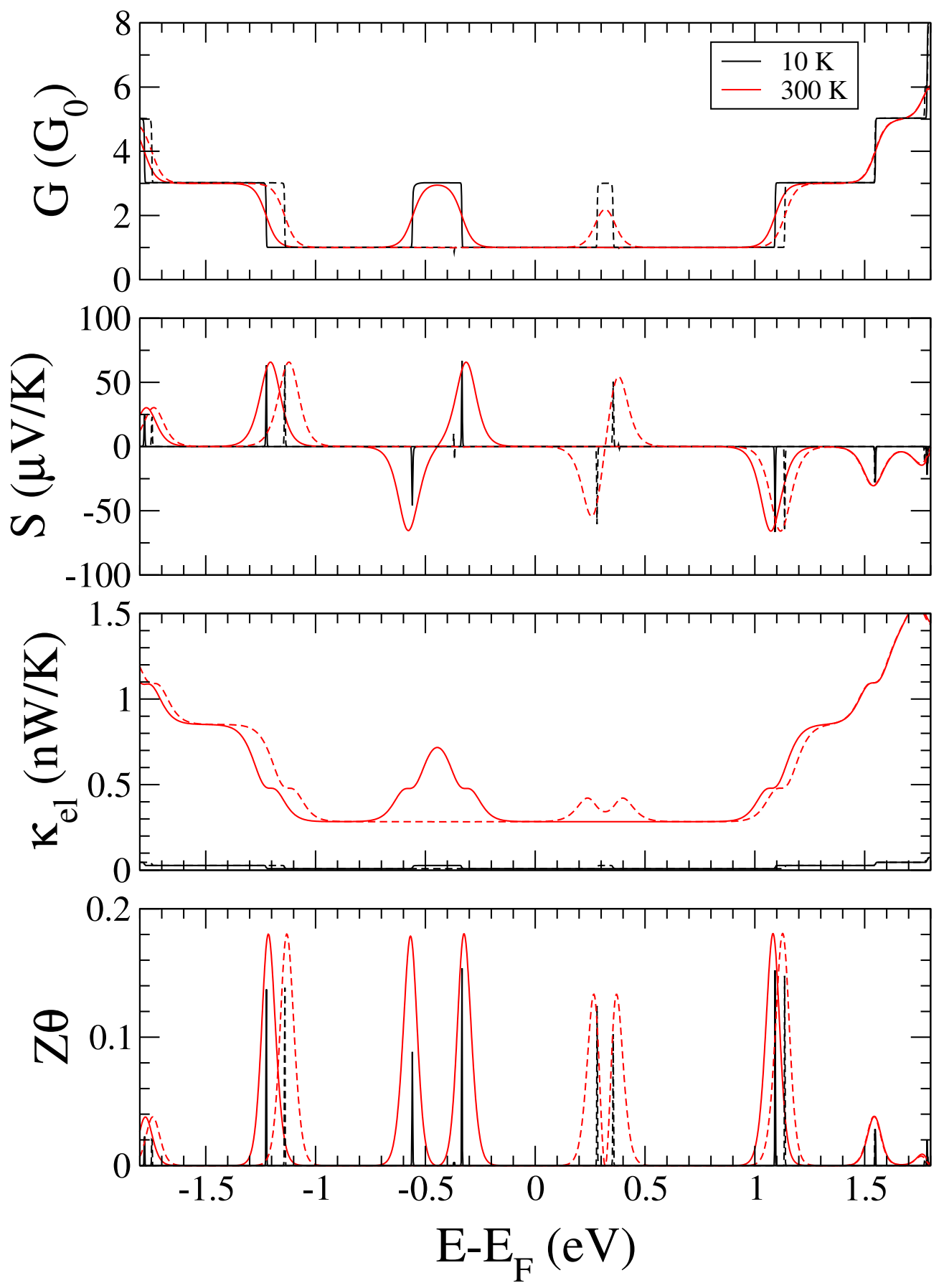

Figura 3.13: Condutância, $G$, coeficiente Seebeck, $S$, condutância térmica eletrônica, $\kappa_{e l}$, e figura de mérito adimensional, $Z \theta$ de uma nanofita $(7,0)$ pristina na configuração ferromagnética para várias temperaturas. 

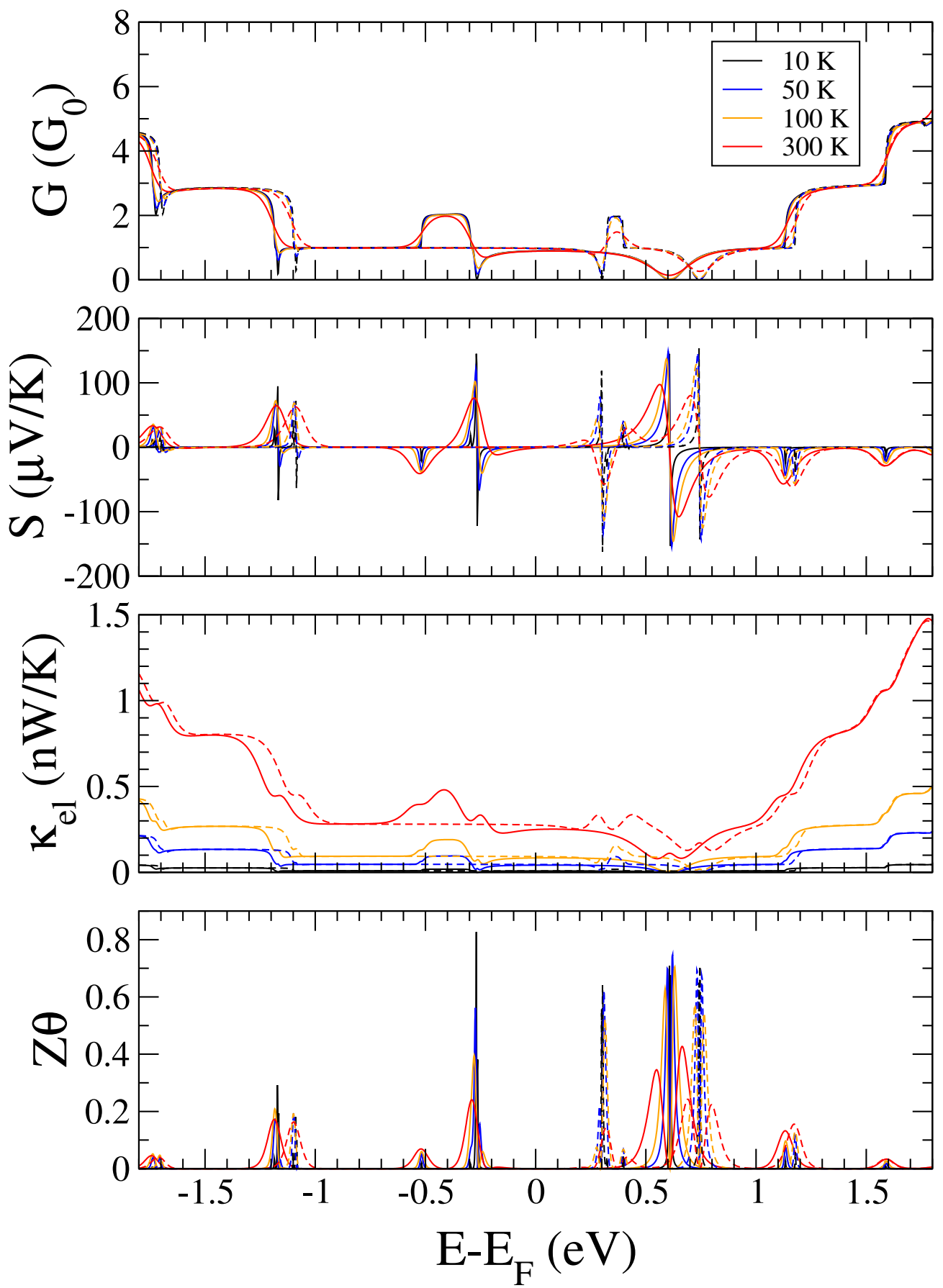

Figura 3.14: Condutância, $G$, coeficiente Seebeck, $S$, condutância térmica eletrônica, $\kappa_{e l}$, e figura de mérito adimensional, $Z \theta$ de uma nanofita $(7,0)$ pristina na configuração ferromagnética com uma impureza de Boro na borda para várias temperaturas. 

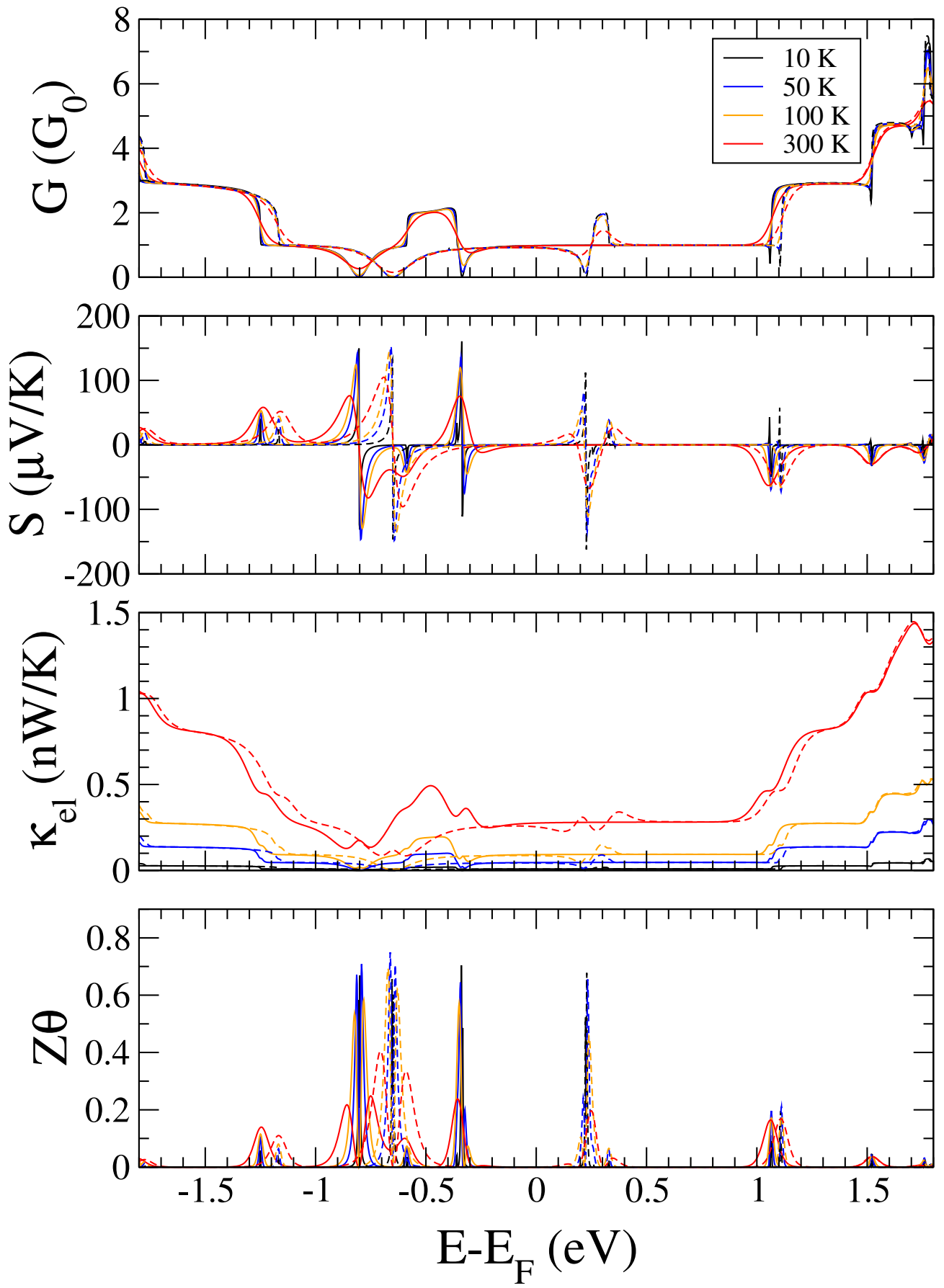

Figura 3.15: Condutância, $G$, coeficiente Seebeck, $S$, condutância térmica eletrônica, $\kappa_{e l}$, e figura de mérito adimensional, $Z \theta$ de uma nanofita $(7,0)$ pristina na configuração ferromagnética com uma impureza de Nitrogênio na borda para várias temperaturas. 


\subsection{Efeito de campo na caloritrônica de spin}

Como vimos até agora, o espalhamento associado aos estados das impurezas de Boro e Nitrogênio causam um aumento do coeficiente Seebeck nas respectivas energias dos defeitos, aproximadamente em torno de $-0.7 \mathrm{e}+0.7 \mathrm{eV}$ para o $\mathrm{N}$ e o $\mathrm{B}$, respectivamente (Figs. 3.15 e 3.14). No entanto, $S=0$ no nível de Fermi tanto para a fita pristina, como para as fitas com B e N. Para poder aproveitar este aumento de $S$ seria necessário deslocar os níveis dos defeitos, que como vimos, são localizados em torno das impurezas (Figs. 3.2 h-i) para as proximidades de $E_{F}$, o que pode ser conseguido através da aplicação de um campo.

Então, a seguir, investigamos o efeito da aplicação de um campo sobre o efeito Seebeck dependente de spin, ou seja, sobre a caloritrônica de spin das nanofitas. O campo é introduzido através da simulação de um potencial de gate em uma região finita dos sistemas. Isto é feito adicionando um potencial eletrostático suave ao hamiltoniano em uma determinada região do espaço no ciclo autoconsistente, o que permite efeitos de blindagem por meio do rearranjo das cargas do sistema [74]. Este potencial consistiu de um platô, $U_{\text {gate }}(z)=e V_{\text {gate }}$ em uma região definida por $z_{0} \leqslant z \leqslant z_{1}$, fazendo uma transição de formato senoidal de largura $\tau$ até a região fora do gate, onde $U_{\text {gate }}(z)=0$ para $z<z_{0}-\tau$ e $z>z_{1}+\tau$.

O efeito do potencial de gate sobre a transmitância nas proximidades do nível de Fermi em uma nanofita pristina é mostrado na Fig. 3.16a. A partir dos potenciais de $+3 \mathrm{eV}$, para o spin $\alpha$, e de $-2 \mathrm{eV}$, para o spin $\beta$, os vales na transmitância se aproximam de $E_{F}$ (lembre-se, da seção 2.3.5, que a largura de $-\partial f / \partial E$ a $300 \mathrm{~K}$ é de $\sim 0.4 \mathrm{eV})$. Estes vales são devidos ao espalhamento causado pelos estados de borda deslocados pelo gate. Na figura 3.16b pode-se ver que a PDOS projetada nos átomos de Carbono das bordas e na região do gate (CBG) coincidem com os vales na transmitância.

A condutância para várias temperaturas em função do potencial de gate é mos- 

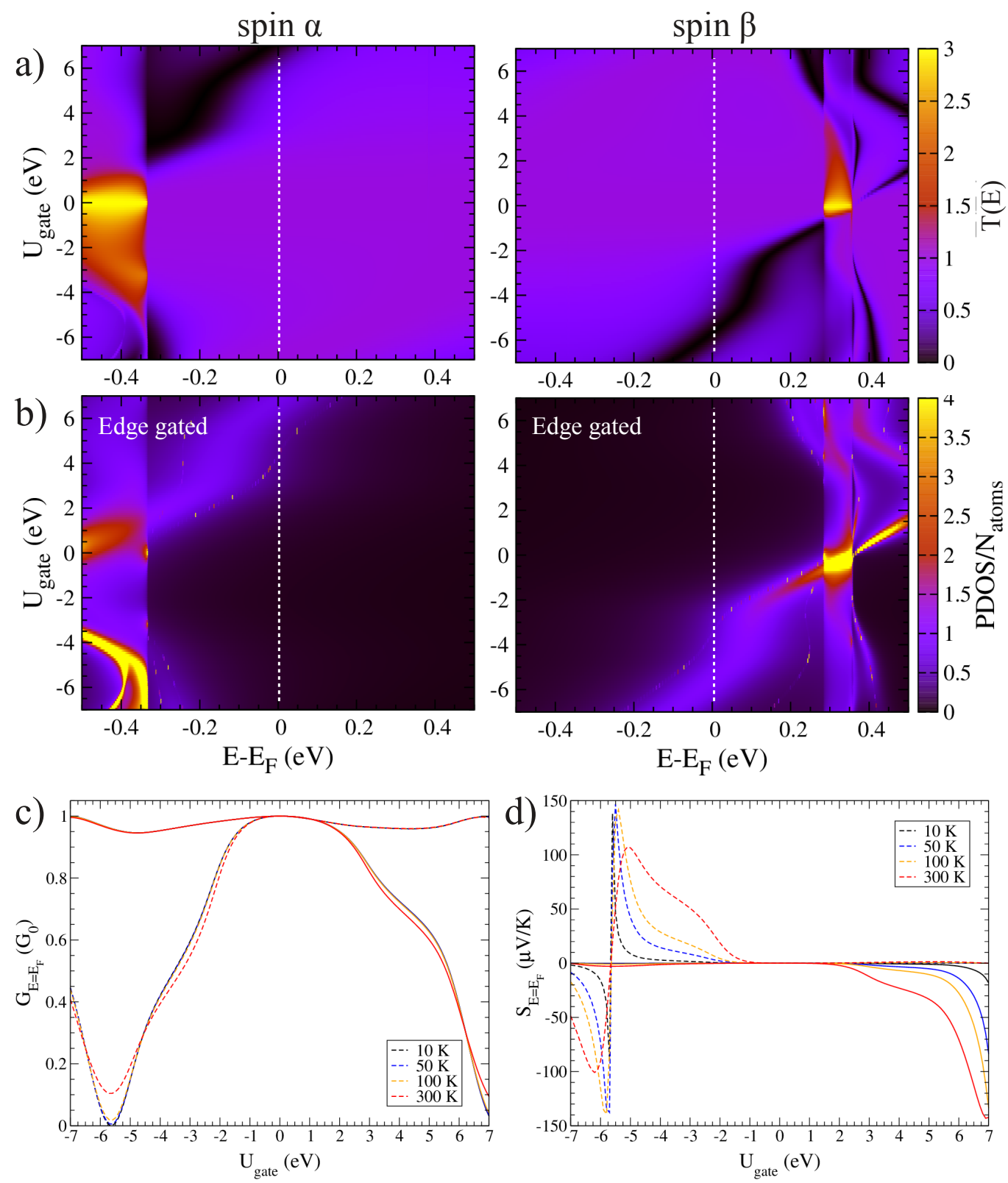

Figura 3.16: Nanofita de grafeno pristina: Mapas de cor da transmitância (a) e da densidade de estados normalizada projetada (PDOS) sobre os átomos de C na borda e sob efeito do gate, em função da energia, $E$, e do potencial de gate, $U_{\text {gate }}$, para os canais de spin $\alpha$ (esquerda) e $\beta$ (direita). Condutância (c) e coeficiente Seebeck (d) no nível de Fermi para várias temperaturas. Em (c-d) o spin $\alpha(\beta)$ é representado por linhas contínuas(tracejadas). 
trado na Fig. 3.16, onde se vê que uma nanofita pristina pode ser usada como filtro de spin. O coeficiente Seebeck (Fig 3.16d) atinge os extremos de $S \approx \pm 100 \mu \mathrm{V} / \mathrm{K}$ para $\theta=300 \mathrm{~K}$ e de $S \approx \pm 140 \mu \mathrm{V} / \mathrm{K}$ para $\theta=10,50$ e $100 \mathrm{~K}$ em torno de $U_{\text {gate }}=-5.5 \mathrm{eV}$ para o canal de $\operatorname{spin} \beta$, enquanto que para o spin $\alpha$ temos $S \approx 0$. Já em $U_{\text {gate }}=+7 \mathrm{eV}$ se dá o contrário, o canal de spin $\alpha$ apresenta $S \approx 0$ e o spin $\beta$, $S \approx-140 \mu \mathrm{V} / \mathrm{K}$. Vemos então, através de um potencial de gate é possível ajustar o coeficiente Seebeck, de forma que a nanofita apresente efeitos termoelétricos apenas para um determinado canal de spin.

Na figura 3.17 apresentamos a transmitância e as PDOS dos átomos de CBG e do Boro. Neste caso, para o canal de spin $\alpha$, a transmitância apresenta dois vales que se aproximam de $E_{F}$ (num intervalo de $\sim 0.2 \mathrm{eV}$ ) a partir de $U_{\text {gate }} \gtrsim 2.5 \mathrm{eV}$, de forma similar à nanofita pristina, e para $U_{\text {gate }} \lesssim-3 \mathrm{eV}$. Estes vales são devidos à espalhamento provocado pelo Boro $\left(U_{\text {gate }} \lesssim-3 \mathrm{eV}\right)$ e pelos átomos de CBG $\left(U_{\text {gate }} \gtrsim\right.$ $2.5 \mathrm{eV}$ e $U_{\text {gate }} \lesssim-3 \mathrm{eV}$ ). Vale notar que como o estado do buraco injetado pelo B se estende por alguns átomos de C na borda (veja a Fig. $3.2 \mathrm{~h}$ ), as contribuições do átomo de $\mathrm{B}$ e dos de CBG para $U_{\text {gate }} \lesssim-3 \mathrm{eV}$ no canal de spin $\alpha$ são devidas ao buraco. Para o canal de spin $\beta$, o vale na transmitância se aproxima de $E_{F}$ a partir de $U_{\text {gate }} \lesssim-2 \mathrm{eV}$ e é devido apenas a átomos de CBG. Conseqüentemente, a condutância (Fig. 3.18a) apresenta um vale no canal de spin $\alpha$ para $U_{\text {gate }}$ positivo e dois vales para $U_{\text {gate }}$ negativo, que cruzam $E_{F}$ em $U_{\text {gate }} \approx-5 \mathrm{eV}(\operatorname{spin} \beta)$ e em $U_{\text {gate }} \approx-7 \mathrm{eV}(\operatorname{spin} \alpha)$.

O coeficiente Seebeck, mostrado na figura $3.18 \mathrm{~b}$, apresenta $S \approx 0$ a partir de $U_{\text {gate }} \gtrsim-2 \mathrm{eV}$ no spin $\alpha$ e os picos associados aos estados de borda $\left(U_{\text {gate }} \gtrsim 2.5 \mathrm{eV}\right)$, que atingem magnitudes de $40 \mu \mathrm{V} / \mathrm{K}$ para 100 e $300 \mathrm{~K}$. Estes valores menores, em comparação à nanofita pristina $(-140 \mu \mathrm{V} / \mathrm{K})$, acontecem por causa do alto valor de $G$ no fundo do vale (em comparação à nanofita pristina, na qual $G \approx 0$ ). Para $U_{\text {gate }} \lesssim-3 \mathrm{eV}$, há uma sobreposição dos picos devidos ao estado do buraco (spin $\alpha$, centrados em torno de $U_{\text {gate }} \approx-7 \mathrm{eV}$ ) e dos estados de borda da fita ( $\operatorname{spin} \beta$, 

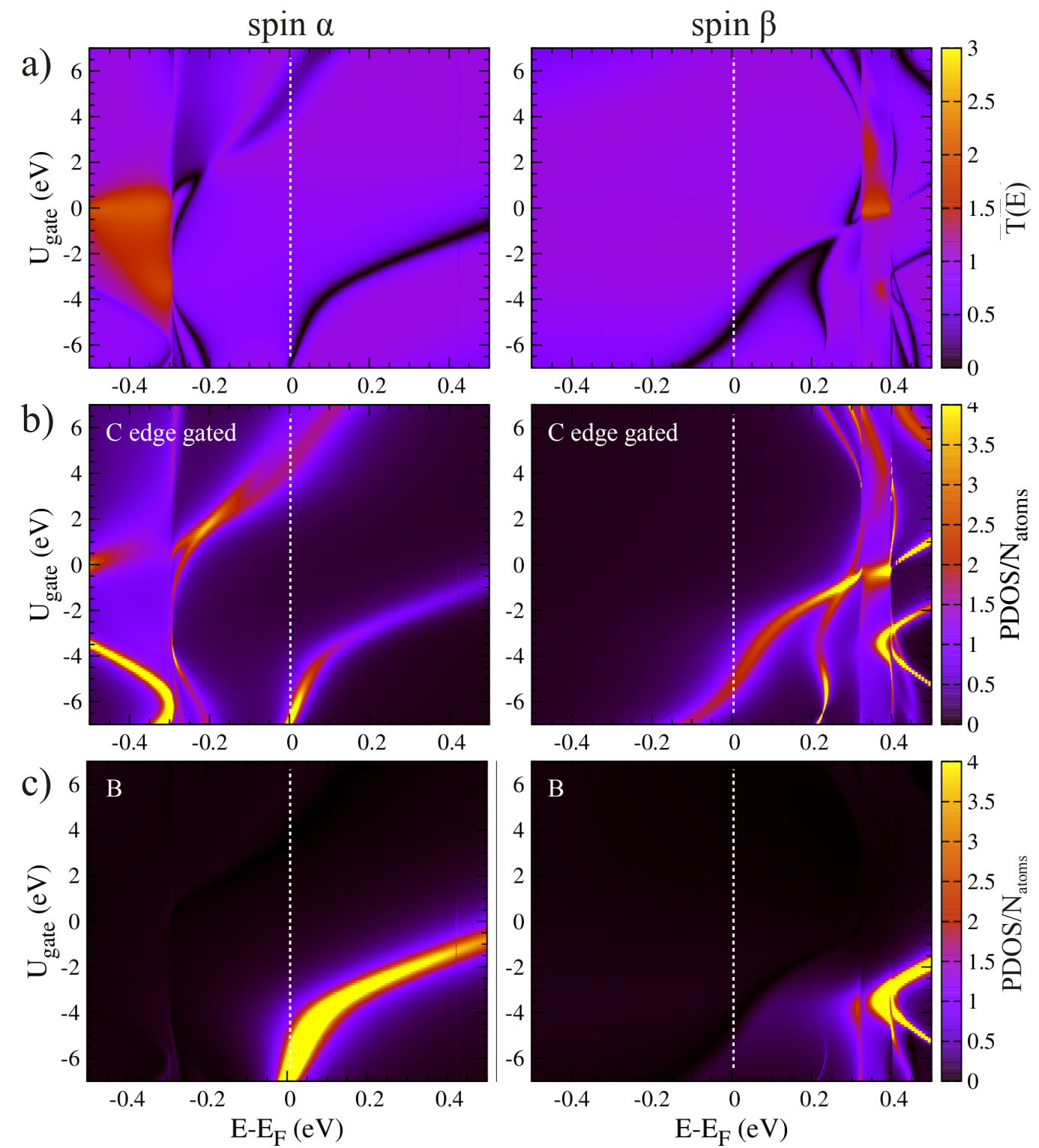

Figura 3.17: Nanofita de grafeno com Boro: Mapas de cor da transmitância (a) e das densidades de estados normalizada projetada (PDOS) sobre os átomos de Carbono na borda e sob efeito do gate (b) e sobre o átomo de Boro (c), em função da energia, $E$, e do potencial de gate, $U_{\text {gate }}$, para os canais de spin $\alpha$ (esquerda) e $\beta$ (direita). 

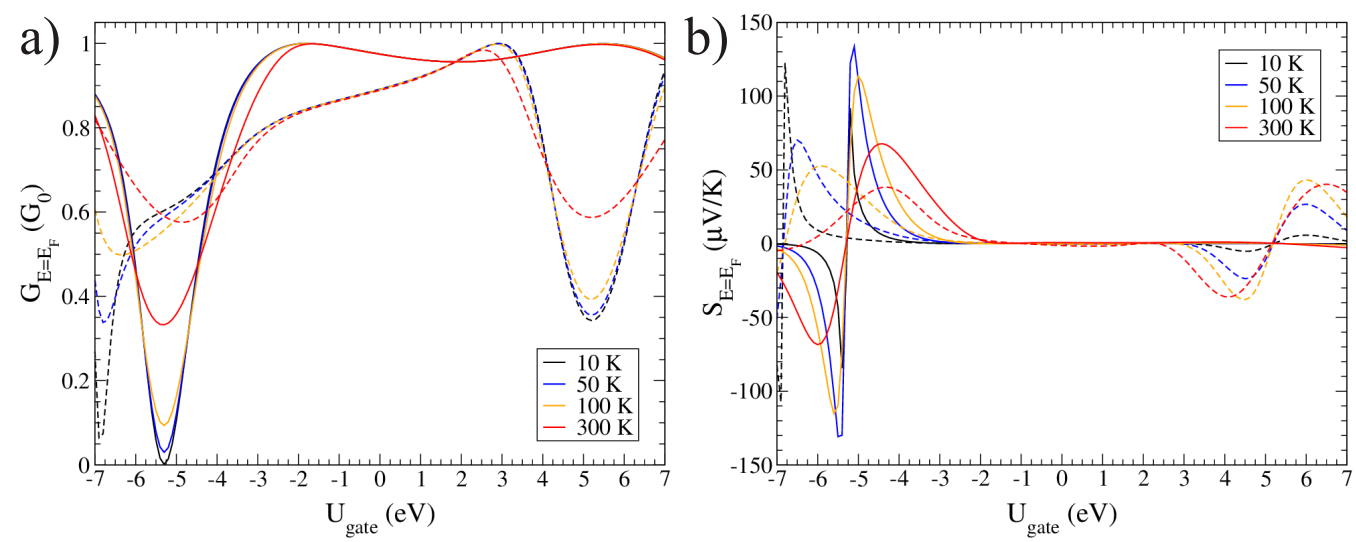

Figura 3.18: Nanofita de grafeno com Boro: Condutância (a) e coeficiente Seebeck (b) no nível de Fermi para várias temperaturas. O spin $\alpha(\beta)$ é representado por linhas contínuas(tracejadas).

centrados em torno de $\left.U_{\text {gate }} \approx-5.5 \mathrm{eV}\right)$.

No caso da nanofita dopada com Nitrogênio, apenas há a inversão da energia do estado do elétron injetado (da mesma forma que nos casos anteriormente analisados). Então, como os mecanismos são os mesmos, apenas mostraremos os resultados encontrados neste caso. Na Fig. 3.19 plotamos a transmitância e as PDOS sobre o átomo de N e sobre os de CBG, e na Fig. 3.20, a condutância e o coeficiente Seebeck para este sistema.

Em suma, vimos que nanofitas de grafeno pristinas e dopadas com Boro e Nitrogênio apresentam efeito termoelétrico dependente do spin e que, ademais, pode ser ajustado via efeito de campo, com a aplicação de um potencial de gate. Contrariamente as expectativas inicias, a nanofita pristina possui um coeficiente Seeebeck consistentemente mais alto do que as nanofitas dopadas, dado os maiores valores de condutância apresentados nos vales em decorrência do estreitamento em energia dos estados do buraco/elétron e da borda quando as fitas são dopadas. 

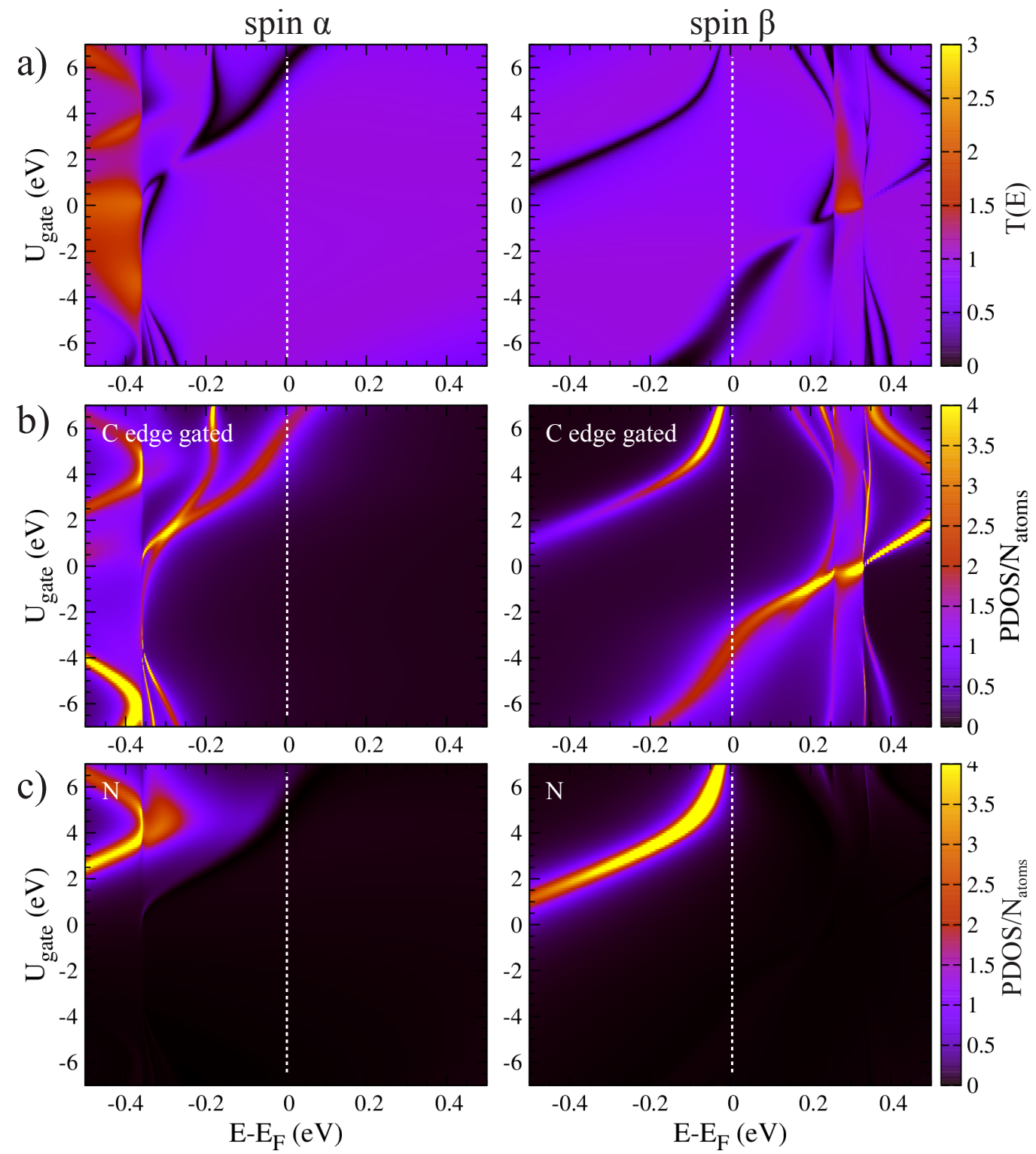

Figura 3.19: Nanofita de grafeno com Nitrogênio: Mapas de cor da transmitância (a) e das densidades de estados normalizada projetada (PDOS) sobre os átomos de Carbono na borda e sob efeito do gate (b) e sobre o átomo de $\mathrm{N}$ (c), em função da energia, $E$, e do potencial de gate, $U_{\text {gate }}$, para os canais de spin $\alpha$ (esquerda) e $\beta$ (direita). 

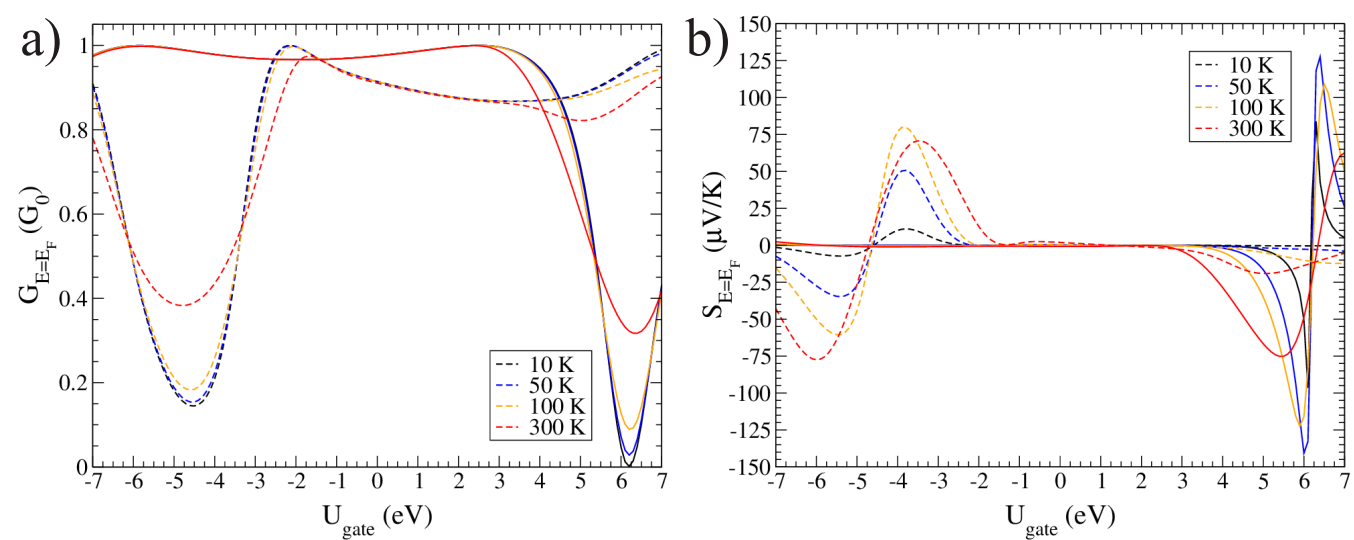

Figura 3.20: Nanofita de grafeno com Nitrogênio: Condutância (a) e coeficiente Seebeck (b) no nível de Fermi para várias temperaturas. O spin $\alpha(\beta)$ é representado por linhas contínuas(tracejadas). 


\section{Capítulo 4}

\section{Bicamada de Grafeno}

Vamos agora apresentar os resultados obtidos no estudo de um dispositivo constituído por uma bicamada de grafeno sob o efeito de potenciais de top gate, $V_{t g}$, e back gate, $V_{b g}$, ambos com comprimento $L_{g a t e}$ (veja a Fig. 4.1). A motivação para estudar este sistema surgiu de um trabalho realizado no grupo SAMPA por J. E. Padilha et al. Ref. [15], no qual as propriedades de transporte de carga foram calculadas por métodos ab initio. Como dito anteriormente (seção 1.3), o fato da bicamada de grafeno poder ter seu gap ajustado por efeito de campo permite que sua resposta termoelétrica também possa ser ajustada pela aplicação de potenciais de gate. Assim, a partir das curvas de transmitância calculadas no grupo para este sistema, demos início a uma investigação das propriedades termoelétricas deste sistema.

Os cálculos foram realizados com o código TRANSAMPA [14, 15], que acopla o formalismo de funções de Green fora do equilíbrio com DFT, através do código SIESTA [70, 71]. Empregamos uma base base duplo- $\zeta$ (DZ), pseudopotenciais de norma conservada [75] e um grid definido por uma energia de corte de 300 Ry. A aproximação de densidade linear (LDA) foi escolhida para o funcional de troca e correlação porque esta descreve corretamente a distância entre as camadas sem a inclusão de correções de van der Waals [76]. A zona de Brillouin foi integrada usando 800 pontos $k_{\perp}$ (direção perpendicular ao plano da bicamada) segundo o esquema de 


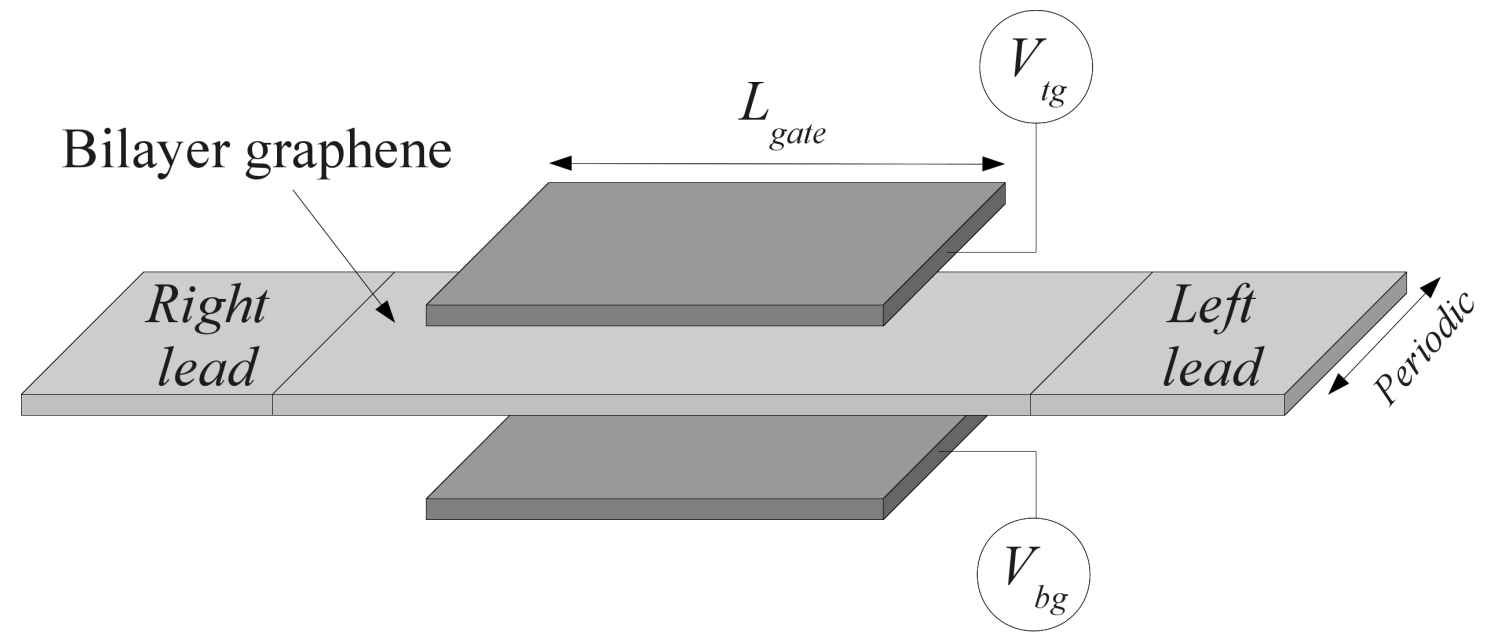

Figura 4.1: Visão esquemática do dispositivo estudado, uma bicamada de grafeno sob o efeito de duas voltagens de gate, $V_{t g}$ e $V_{b g}$.

Monkhorst-Pack [77].

O coeficiente Seebeck e a figura de mérito foram calculados usando as Eqs. (2.61) e 2.63 ,

$$
S=-\frac{1}{e \bar{\theta}} \frac{K_{1}}{K_{0}}
$$

e

$$
Z \theta=\left(\frac{K_{2} K_{0}}{K_{1}^{2}}-1\right)^{-1},
$$

respectivamente, e a resistência foi calculada por $R=1 / G$, onde $G=\left(e^{2} / h\right) K_{0}$ (Eq. (2.60).

Na figura 4.2 apresentamos a transmitância, resistência e coeficiente Seebeck em função da energia de uma bicamada de grafeno sob um gate de comprimento $L_{\text {gate }}=10 \mathrm{~nm}$. A medida que a voltagem de top gate aumenta de $V_{t g}=-4 \mathrm{~V}$ até $V_{t g}=-2 \mathrm{~V}$ o gap causado pelo efeito do campo se desloca para energias maiores. Também é possível notar uma inversão na assimetria dos ramos de elétrons e buracos na transmitância (as regiões de tendência linear). O tamanho do gap também diminui com o aumento de $V_{t g}$, o que pode ser visto claramente pela diminuição do pico nas curvas de resistência (Fig. 4.2b). Através do deslocamento da curva da termopotência, $S(E)$, o valor do coeficiente Seebeck no nível de Fermi 
pode ser ajustado pela voltagem de top gate, passando de valores negativos, onde os portadores são elétrons, até valores positivos, onde os portadores são buracos (Fig. 4.2. c). O valores extremos encontrados são de $S=-243 \mu \mathrm{V} / \mathrm{K}$ em $V_{t g}=-4 \mathrm{~V}$ e de $S=+240 \mu \mathrm{V} / \mathrm{K}$ em $V_{t g}=-3 \mathrm{~V}$. Já no ponto de neutralidade de carga ${ }^{1}$ (indicado pelo círculo aberto) temos $S=-55.7 \mu \mathrm{V} / \mathrm{K}$.

Na figura 4.3 apresentamos $S\left(E_{F}\right)$ em função da voltagem de top gate para temperaturas de 150,200, 250, 300 e $350 \mathrm{~K}$ e um comprimento de gate $L_{\text {gate }}=10 \mathrm{~nm}$. Neste intervalo de temperaturas não há nenhuma mudança muito significativa no perfil das curvas $S\left(E_{F}\right) \times V_{t g}$ embora os valores do coeficiente Seebeck apresentem algumas variações em função da temperatura. Os maiores extremos acontecem para a temperatura de $200 \mathrm{~K}\left(S_{-4 V}=-261 \mu \mathrm{V} / \mathrm{K}\right.$ e $\left.S_{-3 V}=+267 \mu \mathrm{V} / \mathrm{K}\right)$ e os mínimos, para a temperatura de $350 \mathrm{~K}\left(S_{-4 V}=-229 \mu \mathrm{V} / \mathrm{K}\right.$ e $\left.S_{-3 V}=+217 \mu \mathrm{V} / \mathrm{K}\right)$. No ponto de neutralidade de carga, $V_{C N P}\left(V_{t g}=-3.4 \mathrm{~V}\right)$, o coeficiente Seebeck aumenta de forma aproximadamente linear de $-93.9 \mu \mathrm{V} / \mathrm{K}$ em $100 \mathrm{~K}$ até $-45.1 \mu \mathrm{V} / \mathrm{K}$ em 350 K.

A seguir investigamos o efeito do tamanho do gate, $L_{\text {gate }}$, sobre o efeito Seebeck. Como pode ser visto na figura 4.4, o comprimento do gate tem uma grande influência sobre a magnitude do coeficiente Seebeck. Para todos os $L_{\text {gate }}$ calculados o coeficiente Seebeck mínimo se dá na voltagem $V_{t g}=-4 \mathrm{~V}$, passando de $-40.7 \mu \mathrm{V} / \mathrm{K}$ para $L_{\text {gate }}=2 \mathrm{~nm}$ até o valor de $-243 \mu \mathrm{V} / \mathrm{K}$ para $L_{\text {gate }}=10 \mathrm{~nm}$, apresentando uma variação de $597 \%$. A voltagem de top gate na qual o valor máximo de $S\left(E_{F}\right)$ acontece varia entre $V_{t g}=-2 \mathrm{~V} \mathrm{e}-4 \mathrm{~V}$ quando o comprimento do gate varia de 2 até $10 \mathrm{~nm}$, e os valores máximos atingidos vão de $-26.9 \mu \mathrm{V} / \mathrm{K}$ para $L_{\text {gate }}=2 \mathrm{~nm}$ até $+240 \mu \mathrm{V} / \mathrm{K}$ para $L_{\text {gate }}=10 \mathrm{~nm}$, apresentando variação de $892 \%$. Estas grandes variações do valores extremos do coeficiente Seebeck em função do comprimento do gate são dadas pela diminuição do tamanho da barreira induzida quando $L_{\text {gate }}$ diminui, acarretando um gap menor e, conseqüentemente, um menor coeficiente Seebeck em decorrência

\footnotetext{
${ }^{1}$ As voltagens $V_{t g}$ nas quais ocorre o ponto de neutralidade foram obtidas da Ref. [15]
} 

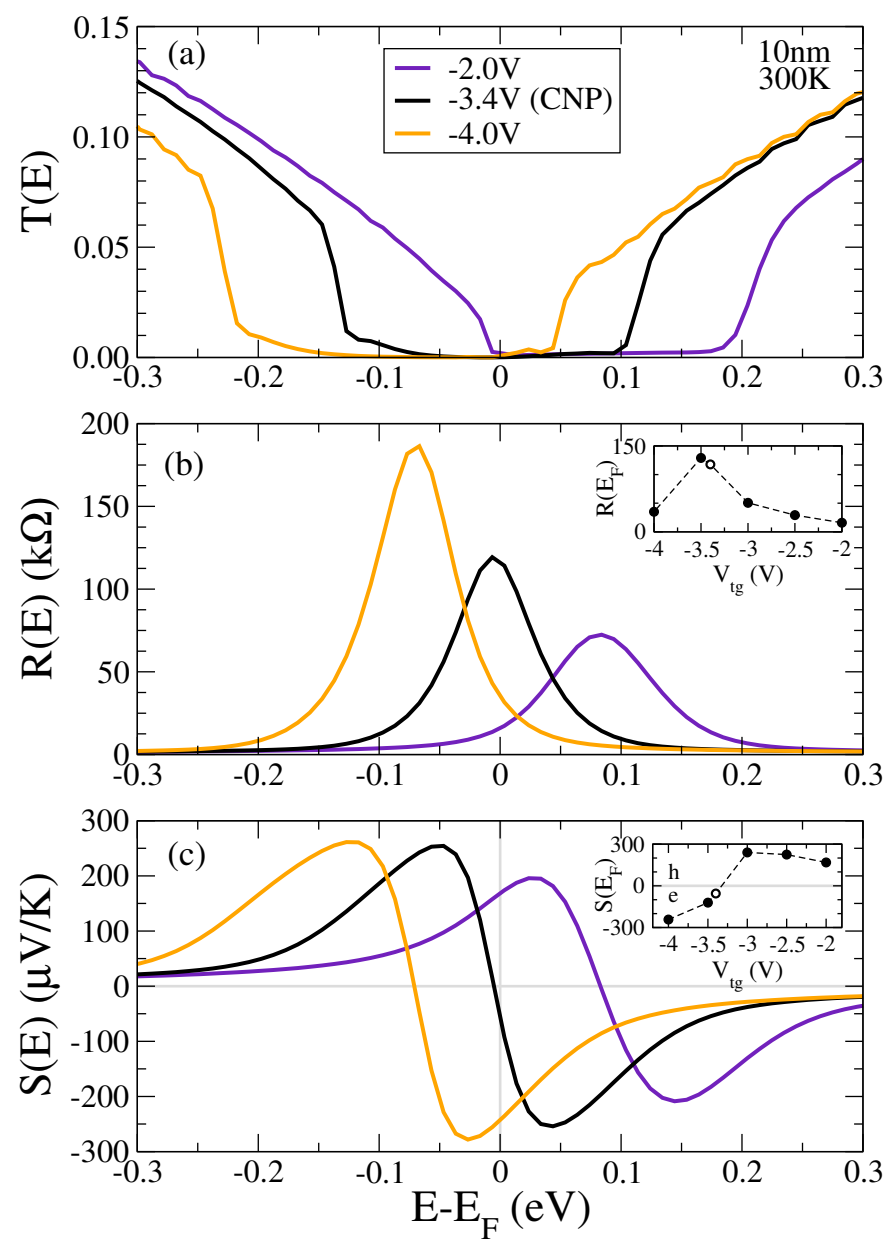

Figura 4.2: (a) Transmitância, $T(E)$, (b) resistência, $R(E)$, e (c) coeficiente Seebeck, $S(E)$, de uma bicamada de grafeno para um gate de comprimento de $10 \mathrm{~nm}$ a temperatura ambiente $(300 \mathrm{~K})$ para três voltagens de gate selecionadas, $V_{t g}$. Nos quadros em (b) e (c), resistividade e coeficiente Seebeck no nível de Fermi, $R\left(E_{F}\right)$ e $S\left(E_{F}\right)$ respectivamente, para todos as voltagens de gate calculadas, onde os círculos abertos indicam o ponto de neutralidade de carga (CNP). Em todos os casos a voltagem de back gate é fixa em $V_{b g}=+2.5 \mathrm{~V}$. 


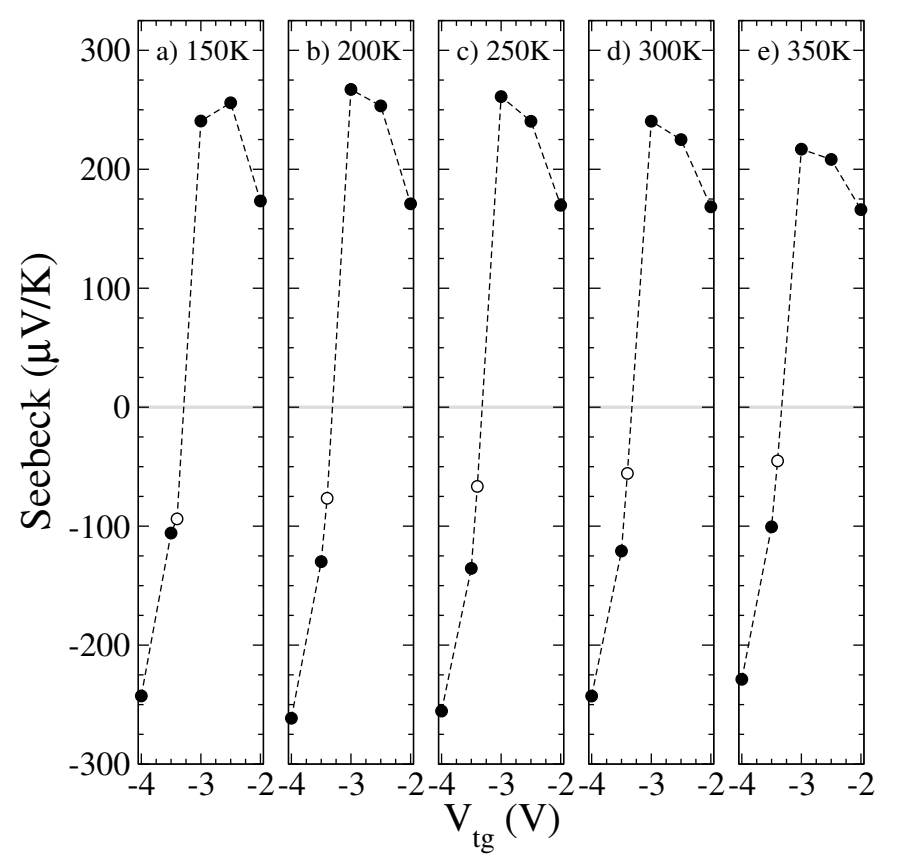

Figura 4.3: Coeficiente Seebeck no nível de Fermi versus voltagem de top gate, $V_{t g}$, para temperaturas de 150 até $350 \mathrm{~K}$, nos gráficos (a) até (e), respectivamente, para um comprimento de gate, $L_{\text {gate }}=10 \mathrm{~nm}$. Os círculos abertos indicam o ponto de neutralidade de carga (CNP). Em todos os casos a voltagem de back gate é fixada em $V_{b g}=+2.5 \mathrm{~V}$. As linhas são apenas guias para a visão. 


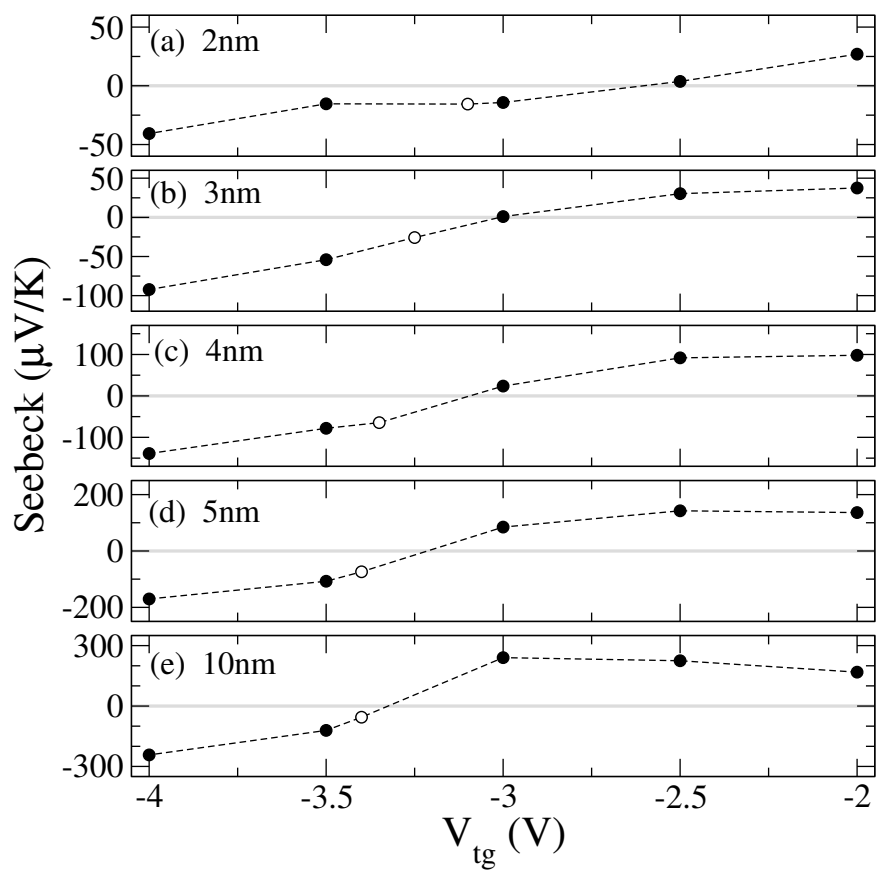

Figura 4.4: Coeficiente Seebeck no nível de Fermi versus voltagem de top gate, $V_{t g}$, para vários comprimentos de gate, $L_{\text {gate }}=2,3,4,5$ e $10 \mathrm{~nm}$ nos gráficos (a) até (e), respectivamente. Os círculos abertos indicam o ponto de neutralidade de carga (CNP). Em todos os casos a voltagem de back gate é fixada em $V_{b g}=+2.5 \mathrm{~V}$ e a temperatura em $300 \mathrm{~K}$. As linhas são apenas guias para a visão. Note as diferentes escalas.

da dependência inversamente proporcional de $S$ com a condutância $G\left(=R^{-1}\right)$. É importante notar que os valores calculados para $S$ concordam qualitativamente (é difícil fazer uma comparação direta devido à dependência de $S$ com $L_{\text {gate }}$ ) com os resultados experimentais (Refs. [54, 53]).

A parte eletrônica da figura de mérito, dada por (Eq. 2.59)

$$
Z T_{e l}=\frac{S^{2} G}{\kappa_{e l}}
$$

e mostrada na figura 4.5 , apresenta um valor quase nulo no ponto de neutralidade de carga, $Z T_{e l}=0.018$. A partir deste ponto, $Z T_{e l}$ aumenta rapidamente a medida que a voltagem de top gate se afasta de $V_{C N P}$, alcançando $Z T_{e l}=2.8 \mathrm{em} V_{t g}=-4 \mathrm{~V}$ e $Z T_{e l}=1.8$ para $V_{t g}=-2.5 \mathrm{~V}$. Estes altos valores da figura de mérito eletrônica são 


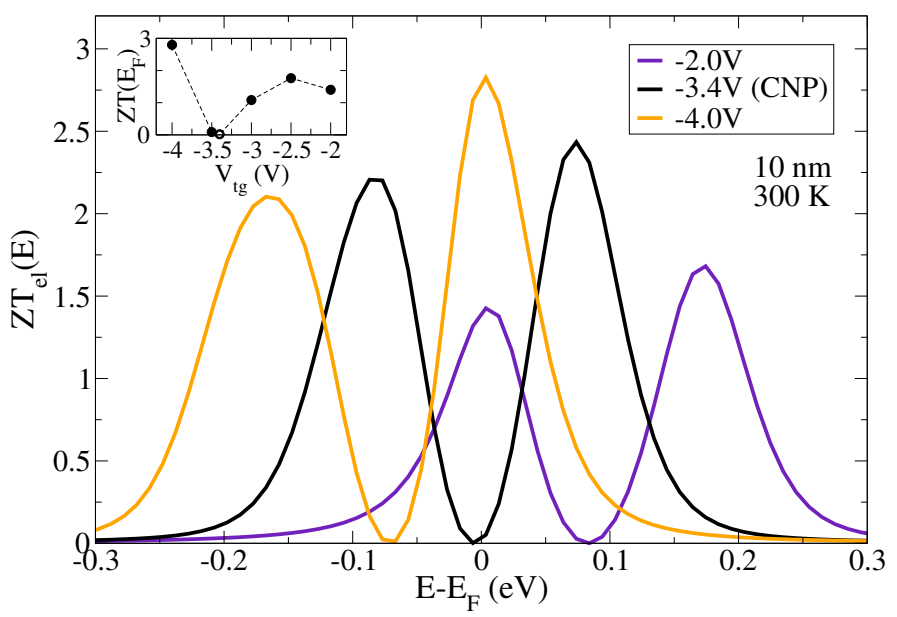

Figura 4.5: Figura de mérito eletrônica, $Z T_{e l}$ de uma bicamada de grafeno para um gate de comprimento de $10 \mathrm{~nm}$ a $300 \mathrm{~K}$ para três voltagens de gate selecionadas, $V_{t g}$. No quadro na figura, $Z T_{e l}$ no nível de Fermi para todos os $V_{t g}$ calculados. Em todos os casos a voltagem de back gate é fixa em $V_{b g}=+2.5 \mathrm{~V}$.

devidos aos picos do coeficiente Seebeck (Fig. 4.2 ), que contribui quadraticamente em $Z T_{e l}$ (Eq. (4.5)). Vale ressaltar que estes valores da figura de mérito são excelentes. No entanto, já que a contribuição da rede na condutância térmica, $\kappa_{L}$, não é contabilizada em $Z T_{e l}$, estes números constituem um limite máximo teórico. Como o grafeno, e materiais grafíticos de forma geral, apresentam $\kappa_{L}$ elevado, calcularemos a seguir esta contribuição para $Z T$.

A condutância térmica da rede foi calculada por dinâmica molecular fora do equilíbrio reversa (RNEMD - Reverse Non-equilibrium Molecular Dynamics) [78]. Neste método, um fluxo de energia é fixado entre as extremidades da caixa de simulação e seu centro (veja a imagem dentro da Fig. 4.6a) através da permutação do momento do átomo mais "quente" (maior energia cinética) das extremidades, com o momento do átomo mais "frio" da faixa central a cada certo número de passos, ou intervalo de tempo, na simulação. O sistema termaliza em resposta a esse fluxo de energia desenvolvendo um perfil de temperatura como o mostrado na Fig. 4.6a.

Segundo a lei de Fourier, a condutividade térmica é definida macroscopicamente 
por

$$
\mathbf{J}=-\kappa \nabla T
$$

onde J é o fluxo de calor. Considerando uma variação de temperatura apenas na direção $z$, temos $J_{z}=-\kappa \partial T / \partial z$. Assim, a condutividade térmica pode ser microscopicamente definida em função de médias temporais,

$$
\kappa=-\lim _{t \rightarrow \infty} \lim _{\partial T / \partial z \rightarrow 0} \frac{\left\langle J_{z}(t)\right\rangle}{\langle\partial T / \partial z\rangle} .
$$

O fluxo de calor é dado por

$$
J_{z}(t)=\Delta E(t) / 2 A t
$$

onde $\Delta E$ é a energia trocada entre as faixas quente e fria, facilmente calculado como

$$
\Delta E=\sum_{\text {transf. }} \frac{m}{2}\left(v_{h}^{2}-v_{c}^{2}\right)
$$

$A$ é a área de seção reta do sistema, $t$ é o tempo de simulação e o fator 2 surge das condições periódicas de contorno (estabelecem-se dois fluxos de calor). Então, depois que o sistema atinge o estado estacionário, a condutividade térmica é calculada como

$$
\kappa=-\frac{\Delta E}{2 A t\langle\partial T / \partial z\rangle}
$$

As simulações de dinâmica molecular foram realizadas com o código LAMMPS [79]. A interação entre os átomos de carbono foi descrita usando o potencial empírico AIREBO [80] e um passo de tempo de 0.5 fs. Foram simuladas bicamadas de grafeno em caixas (usamos condições periódicas de contorno) com largura de $9.7 \mathrm{~nm}$ e comprimentos, $L_{z}$, de 26.8, 53.6, 107 e 214 nm, nas quais o número de átomos variou de 20480 até 163840 . Inicialmente, os sistemas foram termalizados durante 50 
ps (100 000 passos) a temperatura ambiente usando o termostato de Berendsen [81] (ensemble NVT) e calculamos vinte configurações iniciais diferentes para cada $L_{z}$. A seguir, iniciamos a fase de simulação fora do equilíbrio no ensemble NVE, impondo a troca entre as velocidades dos átomos "quentes" e "frios", $v_{h}$ e $v_{c}$ respectivamente, a cada 200 passos. As regiões nas quais a troca ocorre (veja a imagem na Fig. 4.67) foram definidas com um comprimento de $L_{z} / 64$ e o tempo total de simulação nesta fase foi de $500 \mathrm{ps}\left(10^{6}\right.$ passos). Os perfis de temperatura foram obtidos calculando a temperatura em faixas de comprimento $L_{z} / 64$ através de médias temporais das temperaturas locais (tomadas a cada 200 passos), definidas por

$$
T_{k}=\frac{3}{2 N_{i} k_{B}} \sum_{i \in k} m v_{i}^{2}
$$

onde $N_{i}$ é o números de átomos dentro da $k$-ésima faixa e $k_{B}$ é a constante de Boltzmann. $\partial T / \partial z$ é calculado através de um ajuste linear destes perfis de temperatura onde excluímos cinco faixas (com comprimento total $5 L_{z} / 64$ ) adjacentes às faixas quente e fria.

Na figura 4.6 apresentamos o perfil de temperatura obtido (média sobre 20 configurações) para a simulação de comprimento $L_{z}=214 \mathrm{~nm}$ e as condutividades térmicas calculadas. Por meio de uma extrapolação linear de $\kappa^{-1}$ em função de $L_{z}^{-1}$ obtivemos o valor da condutividade térmica no limite de $b u l k, \kappa_{L}=315 \mathrm{~W} / \mathrm{mK}$.

A figura de mérito, incluindo a componente fonônica da condutância térmica, é mostrada na figura 4.7. Como se vê, há uma redução drástica de $Z T$, que passa a ser três ordens de grandeza menor que $Z T_{e l}$. Isto indica que bicamadas de grafeno não são eficientes para aplicações como geradores ou refrigeradores/aquecedores. No entanto, aplicações de outras naturezas, nas quais $Z T$ não seja importante e sim o coeficiente Seebeck, são possíveis, como por exemplo, em dispositivos optoeletrônicos, através do chamado efeito foto-termoelétrico, nos quais os portadores de carga são excitados com luz [82, 83, 84]. 

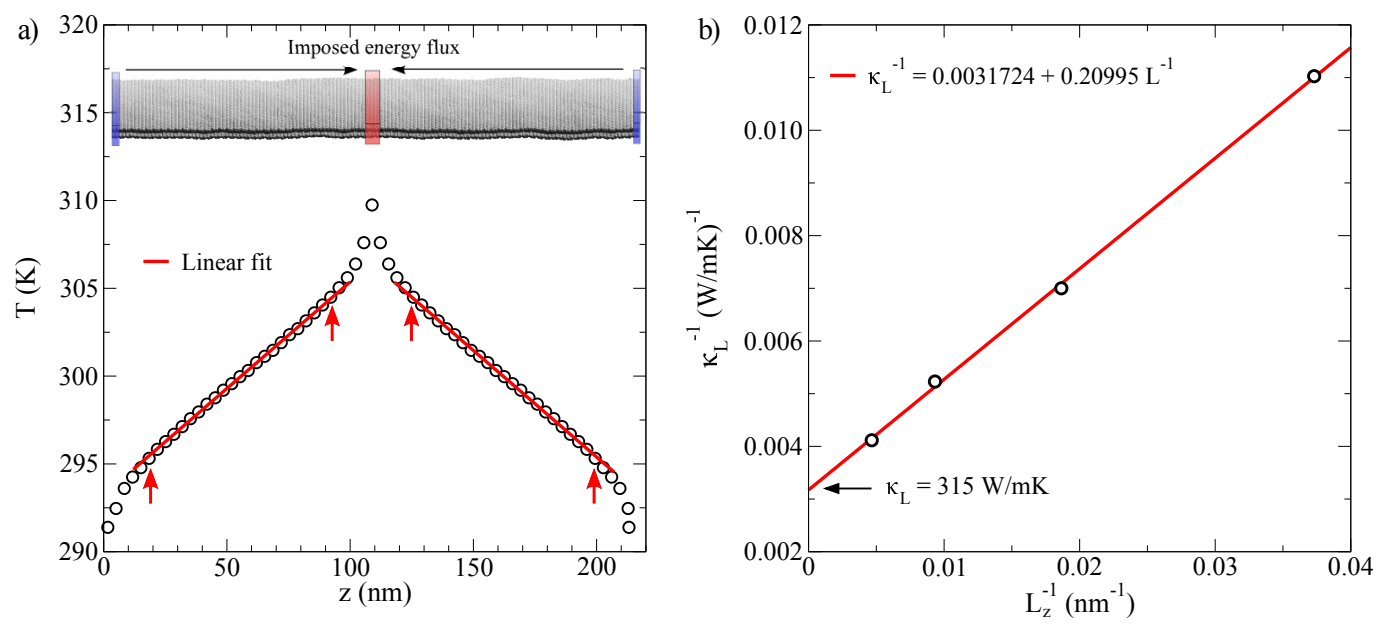

Figura 4.6: (a) Perfil de temperatura típico desenvolvido ao fim de uma simulação. As setas vermelhas indicam o início e fim das regiões usadas para ajustar $\partial T / \partial z$. (b) Inverso da condutividade térmica da rede (fonônica), $\kappa_{L}$, de uma bicamada de grafeno versus inverso do comprimento da caixa de simulação. Todos os valores são médias entre vinte configurações.

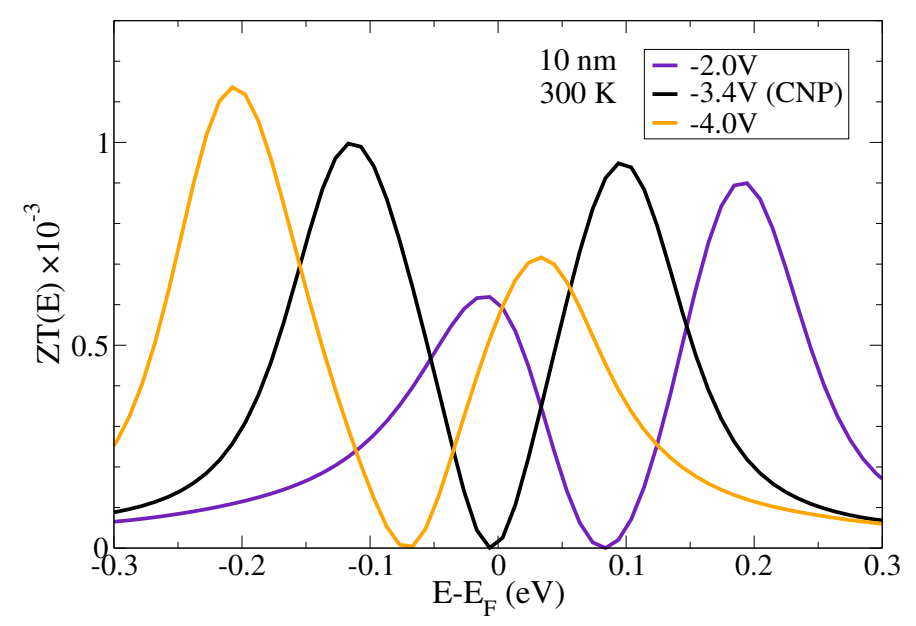

Figura 4.7: Figura de mérito, $Z T$ de uma bicamada de grafeno para um gate de comprimento de $10 \mathrm{~nm}$ a $300 \mathrm{~K}$ para três voltagens de gate selecionadas, $V_{t g}$. No quadro na figura, $Z T$ no nível de Fermi para todos os $V_{t g}$ calculados. Em todos os casos a voltagem de back gate é fixa em $V_{b g}=+2.5 \mathrm{~V}$. 
Em suma, bicamadas de grafeno apresentam efeito Seebeck ajustável por efeito de campo, com o qual $S$ pode ser ajustado de aproximadamente -250 até $+250 \mu \mathrm{V} / \mathrm{K}$, valores de magnitude comparável ao de nanofios de Si [85, 86]. Embora a parte eletrônica da figura de mérito seja alta (até 2.8), quando a contribuição da condutividade térmica da rede é contabilizada obtém-se $Z T \sim 10^{-3}$. Portanto, bicamadas de grafeno são eficientes apenas em aplicações nas quais o valor do coeficiente Seebeck (ou sua ajustabilidade) sejam mais importantes que $Z T$. 


\section{Capítulo 5}

\section{Caloritrônica de spin em Grafeno}

\section{com Mn}

O campo da caloritrônica de spin lida com a interação entre correntes de calor e de spin, isto é, o acoplamento entre spintrônica e termoeletricidade [87]. O grafeno [19, 23] é um material com grande potencial para uso em dispositivos spintrônicos porque apresenta um longo caminho médio e acoplamento spin-órbita fraco [88, 89], preservando a coerência de spin dos elétrons. Ademais, o grafeno também já foi proposto como material para dispositivos termoelétricos, tanto teoricamente [90, 91] como experimentalmente [92, 83, 24]. Assim, pelo menos em princípio, o grafeno seria um bom candidato para realizar caloritrônica de spin. Dispositivos baseados em nanofitas de grafeno zigzag já foram propostos para este fim [73, 93, 94, 95]. No entanto, sintetizar nanofitas de grafeno (1D) com bordas bem definidas na direção zigzag (ou qualquer direção específica, na verdade) é uma tarefa muito complicada do ponto de vista experimental [96]. O grafeno bidimensional, por si só, embora seja um excelente condutor para correntes polarizadas, é degenerado em spin e portanto não pode ser utilizado como uma fonte de correntes com polarização de spin. Uma forma de levantar sua degenerescência em spin que tem sido investigada ultimamente é dopando o grafeno com metais [97, 98, 99, 100, 101, 74]. Embora a tendência geral 
seja a que os metais se difundam sobre o grafeno e formem agregados, eles podem ser aprisionados em defeitos como monovacâncias (MV), onde permanecem imóveis e são altamente estáveis [102, 103, 74]. Monovacâncias em grafeno podem ser criadas por irradiação de íons [104] ou elétrons [97] e, neste último caso, o uso de feixes focalizados permite controle espacial sub-nanométrico [105] na criação dos defeitos.

Entre os vários metais já estudados na literatura, o manganês, Mn, apresenta características ótimas quando preso a uma vacância (Mn@MV) em grafeno. O Mn se liga fortemente à MV, com uma energia de ligação de $-6 \mathrm{eV}$ e apresenta um momento magnético de $3 \mu_{B}$, resultante de elétrons desemparelhados nos orbitais $3 d$ do Mn. Estes orbitais são localizados no Mn e tem energias próximas ao nível de Fermi [98, 74, 101]. Em um trabalho realizado no grupo (ref. [74]) já foi mostrado que este sistema pode ser usado para criar correntes polarizadas que podem ser ajustadas com um potencial de gate, $V_{g}$. Desta forma, é natural se perguntar se este sistema também apresentaria propriedades termoelétricas dependentes de spin. Neste trabalhd $\rrbracket^{1}$ adereçamos esta questão calculando o coeficiente Seebeck e as correntes em um dispositivo formado por grafeno com um Mn@MV sobre o efeito de um potencial de gate ligado a contatos mantidos a temperaturas distintas (veja a Fig. 5.1. A).

Os cálculos foram realizados com o formalismo de funções de Green acoplado à teoria do funcional da densidade. Primeiramente, a geometria, composta de uma supercélula de tamanho $13.0 \AA \times 20.0 \AA \times 32.5 \AA$, foi completamente relaxada usando um critério de força de $0.02 \mathrm{eV} / \AA$ empregando o código SIESTA [70, 71]. A seguir, as transmitâncias foram calculadas usando o TRANSAMPA2 2] 14, 15]. Em todos os cálculos adotamos a aproximação de gradiente generalizado de Perdew-BurkeErnzerhof [106] para o funcional de troca e correlação, pseudopotenciais de norma conservada [75] e uma base duplo- $\zeta$ polarizada (DZP). Usamos uma energia de corte

\footnotetext{
${ }^{1}$ Realizado em colaboração com o Dr. Matheus Paes Lima a partir de um dos sistemas estudados na ref. 74

${ }^{2}$ Esta versão foi modificada pelo autor para permitir o uso de placas de processamento gráficas (GPUs) para acelerar os cálculos. Veja o apêndice E.
} 


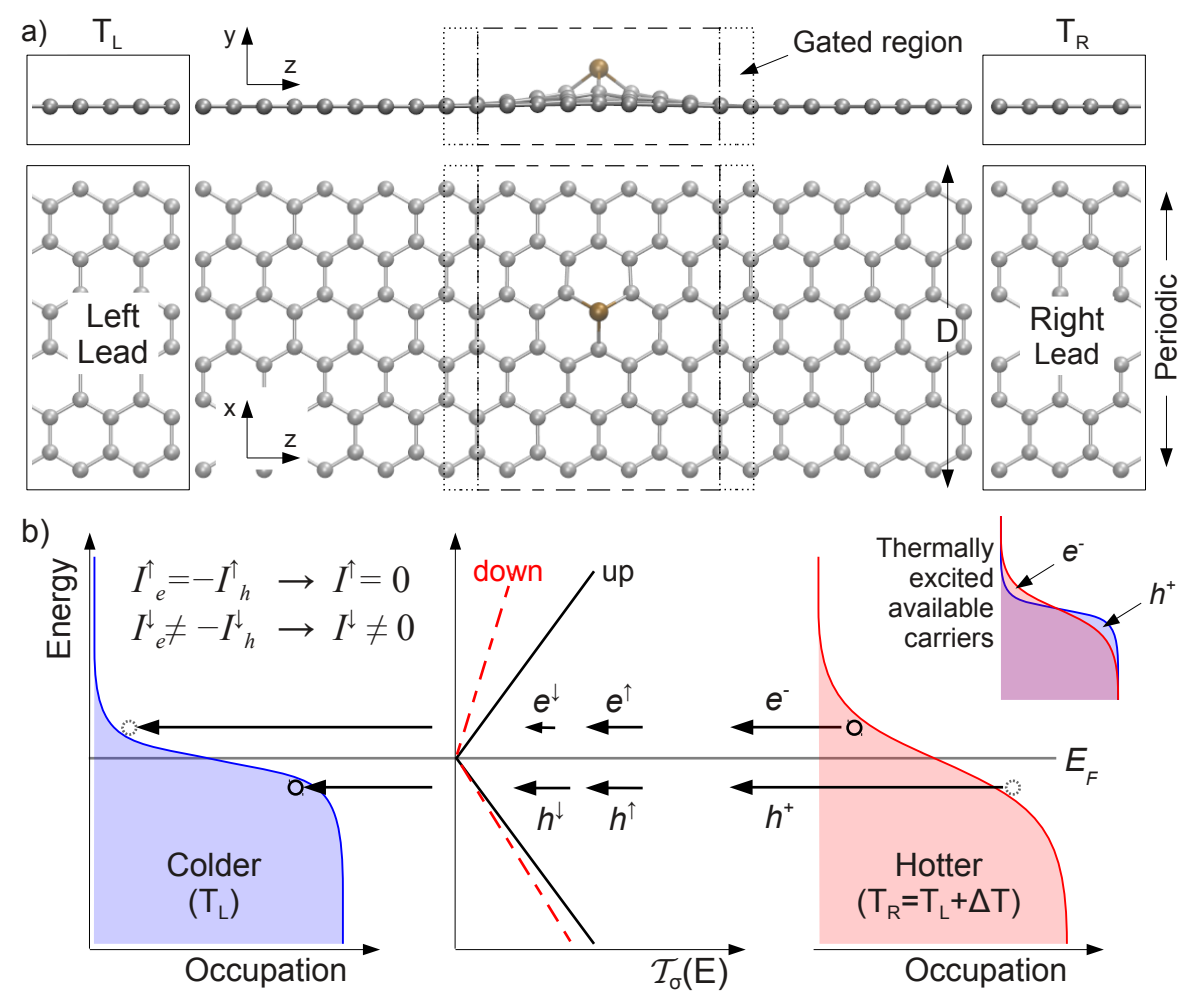

Figura 5.1: (a) Geometria do sistema estudado, uma folha de grafeno (periódica na direção $x$ ) com um átomo de Mn preso em uma monovacância, acoplado a contatos de grafeno pristino. A região indicada por linhas tracejadas está sob o efeito de um potencial de gate, $V_{g}$. (b) Diagrama esquemático do mecanismo físico do efeito Seebeck dependente de spin.

para grid (mesh cutoff) de 300 Ry e uma amostragem de $7 \times 1 \times 4$ pontos $k$, no esquema de Monkhorst-Pack[77], para integrar a zona de Brillouin. Para os cálculos de transporte eletrônico, empregamos 201 pontos $k$ (3001 para o cálculo da PDOS) na direção perpendicular ao transporte (direção $x$ na Fig. 5.1a). O potencial de gate, $V_{g}$, foi simulado adicionando um potencial eletrostático suave ao Hamiltoniano em uma região finita do espaço (uma "fatia" perpendicular à direção $z$ contendo o Mn@MV) de forma autoconsistente, permitindo assim efeitos de blindagem através do rearranjo de cargas [74]. Este potencial consiste de um platô, $U(z)=e V_{g}$, em uma dada região dada por $z_{\min }<z<z_{\max }$ (representada pela linha tracejada na Fig. 5.1 a) e "escadas" com forma senoidal de largura $\tau$ (região indicada por linhas pontilhadas na Fig. 5.1 a); $U(z)=0$ para $z<z_{\min }-\tau$ e $z>z_{\max }+\tau$. Nos cálculos 
definimos a região com potencial de gate como $z_{\text {min }}=11.24 \AA$, $z_{\text {min }}=21.23 \AA$ (platô de largura de $10 \AA$ centrado na posição do Mn) e $\tau=1.25 \AA$. Uma camada de vácuo de $20 \AA$ foi usada para evitar interações espúrias entre imagens periódicas na direção perpendicular ao plano do grafeno ( $y$ na Fig. 5.1 a).

No formalismo de Landauer a corrente é dada por [107]

$$
I_{\sigma}=\frac{e}{h} \int \mathcal{T}_{\sigma}\left(E ; V_{g}\right)\left(f_{L}-f_{R}\right) d E,
$$

onde $\sigma=\uparrow, \downarrow$ é o spin, e é a carga do elétron, $\mathcal{T}_{\sigma}\left(E ; V_{g}\right)$ é a transmitância dependente de $V_{g}, f_{L(R)} \equiv f\left(E, \mu, T_{L(R)}\right)$ é a função de distribuição de Fermi-Dirac, $\mu$ é potencial eletroquímico e $T_{L(R)}$ é a temperatura do eletrodo esquerdo (direito).

Quando os contatos são mantidos a temperaturas diferentes, o desbalanço resultante na densidade de portadores de carga termicamente excitados, determinado por $f_{L}-f_{R}$, permite que elétrons $\left(e^{-}\right)$e buracos $\left(h^{+}\right)$estejam disponíveis para fluir do eletrodo quente para o frio, como mostrado na figura 5.1 b. No entanto, para que as correntes de $e^{-}$e $h^{+}$não se cancelem mutuamente e que uma corrente líquida possa ser estabelecida é necessário que $\mathcal{T}_{\sigma}\left(E ; V_{g}\right)$ seja assimétrica em torno do nível de Fermi, $E_{F}$. Isto é, a transmitância para $e^{-}\left(\mathcal{T}_{\sigma}^{e}\right)$ e para $h^{+}\left(\mathcal{T}_{\sigma}^{h}\right)$ tem que ser diferente, caso contrário $I_{\sigma}^{e}+I_{\sigma}^{h}=0$.

O coeficiente Seebeck dependente de spin é calculado como

$$
S_{\sigma}=-\frac{1}{e \bar{T}} \frac{\int \mathcal{T}_{\sigma}\left(E ; V_{g}\right)\left(-\frac{\partial f}{\partial E}\right)(E-\mu) d E}{\int \mathcal{T}_{\sigma}\left(E ; V_{g}\right)\left(-\frac{\partial f}{\partial E}\right) d E},
$$

onde $\bar{T}$ é a temperatura média dos eletrodos. Note que o numerador da Eq. 5.2 (e conseqüentemente $S_{\sigma}$ ) é uma medida da assimetria local da transmitância em torno de $\mu$ em uma janela de energia dada por $\partial f / \partial E$ (cuja largura é determinada pela temperatura $\bar{T}$ ). Veja também que se $\mathcal{T}_{\sigma}^{e}(E)$ for maior (menor) que $\mathcal{T}_{\sigma}^{h}(E)$ (próximo ao nível de Fermi), o $S_{\sigma}$ resultante será negativo (positivo). 

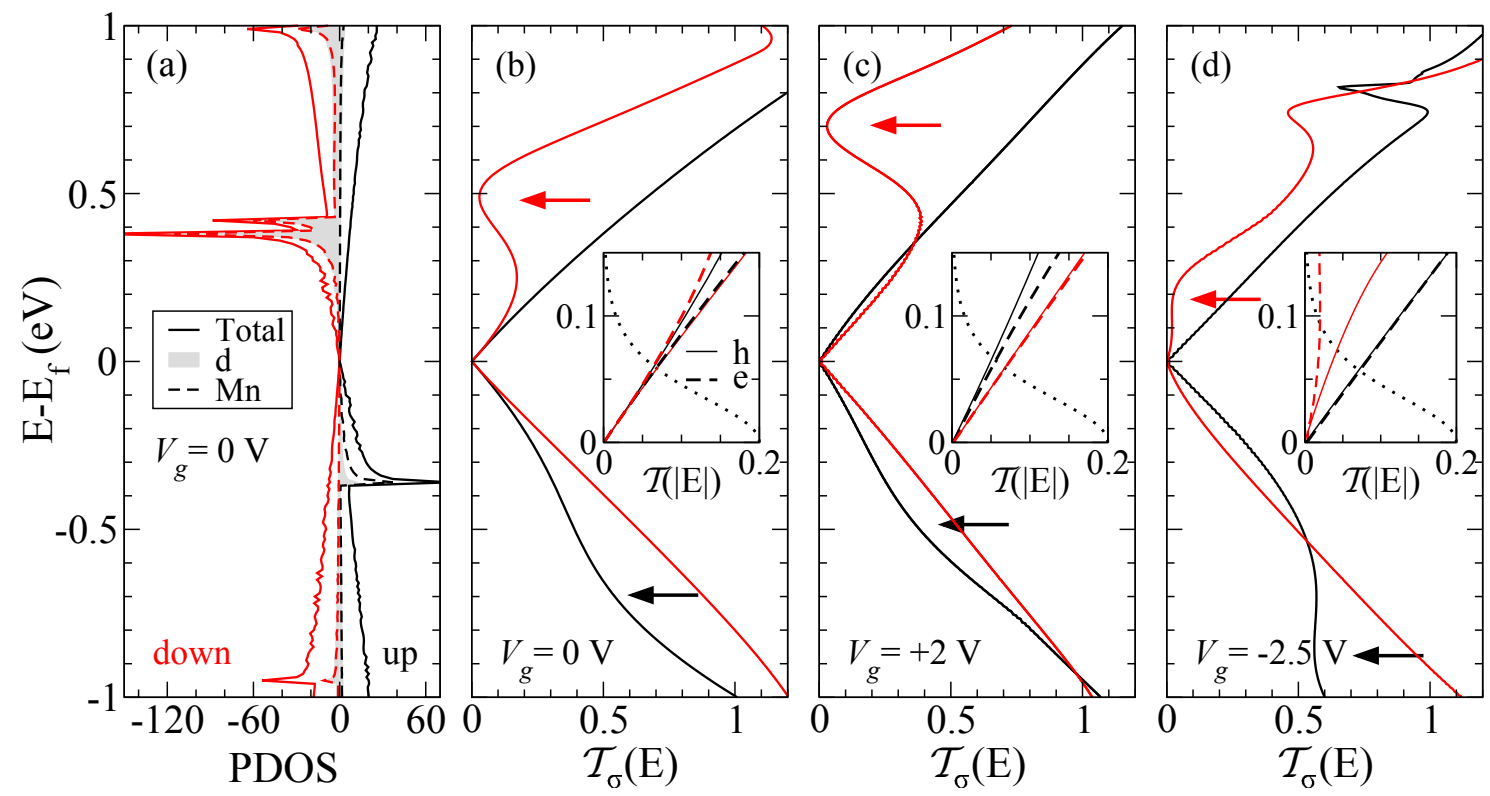

Figura 5.2: (a) Densidade de estados projetada (PDOS) de Mn@MV em grafeno para $V_{g}=0$. (b-d) Transmitâncias, $\mathcal{T}_{\sigma}(E)$, para os potenciais de gate de $V_{g}=0$, $+2 \mathrm{e}-2.5 \mathrm{~V}$, respectivamente. As setas indicam os vales em $\mathcal{T}_{\sigma}(E)$ associados aos estados $d$ do Mn. Nos quadros em (b-d), detalhe de $\mathcal{T}_{\sigma}(E)$ para $e^{-}$e $h^{+}$. As linhas pontilhadas representam $-\partial f / \partial E$ a $300 \mathrm{~K}$ (unidades arbitrárias). Em todos os casos $T_{L}=T_{R}=300 \mathrm{~K}$.

Na Fig. 5.2 a, mostramos a densidade de estados projetada (PDOS - Projected density of states) em todo o sistema, no átomo de Mn e nos orbitais $d$ (veja a Fig. 5.1 ) $\left(V_{g}=0\right.$ e $\left.D=13 \AA\right)$. Como pode ser visto, o átomo de Mn preso em um monovacância apresenta estados $d$ localizados ocupados (desocupados) simetricamente localizados a aproximadamente $-(+) 0.4 \mathrm{eV}$ de $E_{F}$ para os spins $\uparrow(\downarrow)$. Estes estados dão origem a vales em $\mathcal{T}_{\sigma}(E)$, indicados por setas na Fig. 5.2 , que também afetam a inclinação da transmitância nas proximidades de $E_{F}$. Voltagens de gate positivas (Fig. 5.2 c) levantam os orbitais $d$, movendo os vales nos canais de spin $\uparrow(\downarrow)$ para perto (longe) de $E_{F}$. Por outro lado, $V_{g}$ negativas (Fig. 5.2d) abaixam os níveis $d$, deslocando os vales $\uparrow(\downarrow)$ para longe (perto) de $E_{F}$. Nos quadros das Figs. $5.2 \mathrm{~b}-\mathrm{d}$ apresentamos em mais detalhe a assimetria em $\mathcal{T}_{\sigma}(E)$ entre $e^{-}\left(E>E_{F}\right)$ e $h^{+}\left(E<E_{F}\right)$ nas proximidades do nível de Fermi. Para $V_{g}=0$ ambos os canais de spin são simétricos para $|E| \lesssim 0.05 \mathrm{eV}$. No entanto para $|E| \gtrsim 0.05 \mathrm{eV}, \mathcal{T}_{\uparrow}^{e}>\mathcal{T}_{\uparrow}^{h}$ 
e $\mathcal{T}_{\downarrow}^{e}<\mathcal{T}_{\downarrow}^{h}$ (resultando em $S_{\uparrow}<0$ e $S_{\downarrow}>0$ ). Neste caso, é interessante notar que $\mathcal{T}_{\uparrow(\downarrow)}^{e(h)} \approx \mathcal{T}_{\downarrow(\uparrow)}^{h(e)}$, o que significa que (aproximadamente) não há transporte de carga líquido porque as correntes de $e^{-} \mathrm{e} h^{+}$resultantes se cancelam mutuamente, $I^{e} \approx I^{h}$, mas há corrente de spin porque este cancelamento não é entre portadores com mesmo spin. Para $V_{g}=2 \mathrm{~V}$, o vale para o canal de spin $\downarrow$ é afastado de $E_{F}$, tornando $\mathcal{T}_{\downarrow}^{e(h)}$ simétrica e, conseqüentemente $S_{\downarrow}=0$. Já o vale para o canal de spin $\uparrow$ está próximo de $E_{F}$, diminuindo $\mathcal{T}_{\uparrow}$ para $E>E_{F}$, ou seja, tornando $\mathcal{T}_{\uparrow}^{e}>\mathcal{T}_{\uparrow}^{h}$ e, portanto, $S_{\uparrow}<0$. O comportamento contrário acontece para $V_{g}=-2.5 \mathrm{~V}$, o afastamento do vale no canal de spin $\uparrow$ de $E_{F}$, torna $\mathcal{T}_{\uparrow}$ simétrica entre $e^{-}$e $h^{+}$ (e $S_{\uparrow}=0$ ), enquanto que a aproximação do vale no canal de spin $\downarrow$ de $E_{F}$ deixa $\mathcal{T}_{\downarrow}^{e}<\mathcal{T}_{\downarrow}^{h}$, resultando em $S_{\downarrow}>0$.

A diferença de energia total entre os alinhamentos ferromagnético (F) e antiferromagnético (AF) em função da distância Mn-Mn, $d$, é mostrada no quadro da Fig. 5.3 a. Para realizar esta simulação a geometria mostrada na Fig. 5.17a foi lateralmente duplicada, resultando em uma supercélula com dois Mn@MV e $D=2 d$. Vemos que, para $d \lesssim 23 \AA$ a configuração AF é energeticamente favorável, indicando que um campo magnético seria necessário para obter a configuração F. No entanto, para $d \gtrsim 23 \AA$ o alinhamento F é o mais favorável energeticamente. Note que apenas a configuração F possui o desbalanço de spin necessário para gerar correntes polarizadas em spin. Assim, todos os resultados apresentados aqui, com exceção da magnetorresistência termicamente induzida consideram este tipo de alinhamento.

Na figura 5.3, mostramos como o coeficiente Seebeck e a corrente termicamente induzida variam com $V_{g}$ para $D=13 \AA$ e $D=26 \AA$. Para o maior $D$, a dispersão (e o alargamento) dos estados localizados $d$ diminuem, resultando em vales mais estreitos em $\mathcal{T}_{\sigma}(E)$ [74]. Contudo, o comportamento qualitativo é o mesmo em ambos os casos: $V_{g}$ desloca os os vales em $\mathcal{T}_{\sigma}$, o que permite ajustar sua assimetria entre $e^{-}$e $h^{+}$e, conseqüentemente, ajustar $S_{\sigma}$ e $I_{\sigma}$. Da Fig. 5.3 podem ser facilmente identificados três intervalos de $V_{g}$, que nomeamos de $V_{-}, V_{0}$ e $V_{+}$, nos quais o sistema é ajustado 


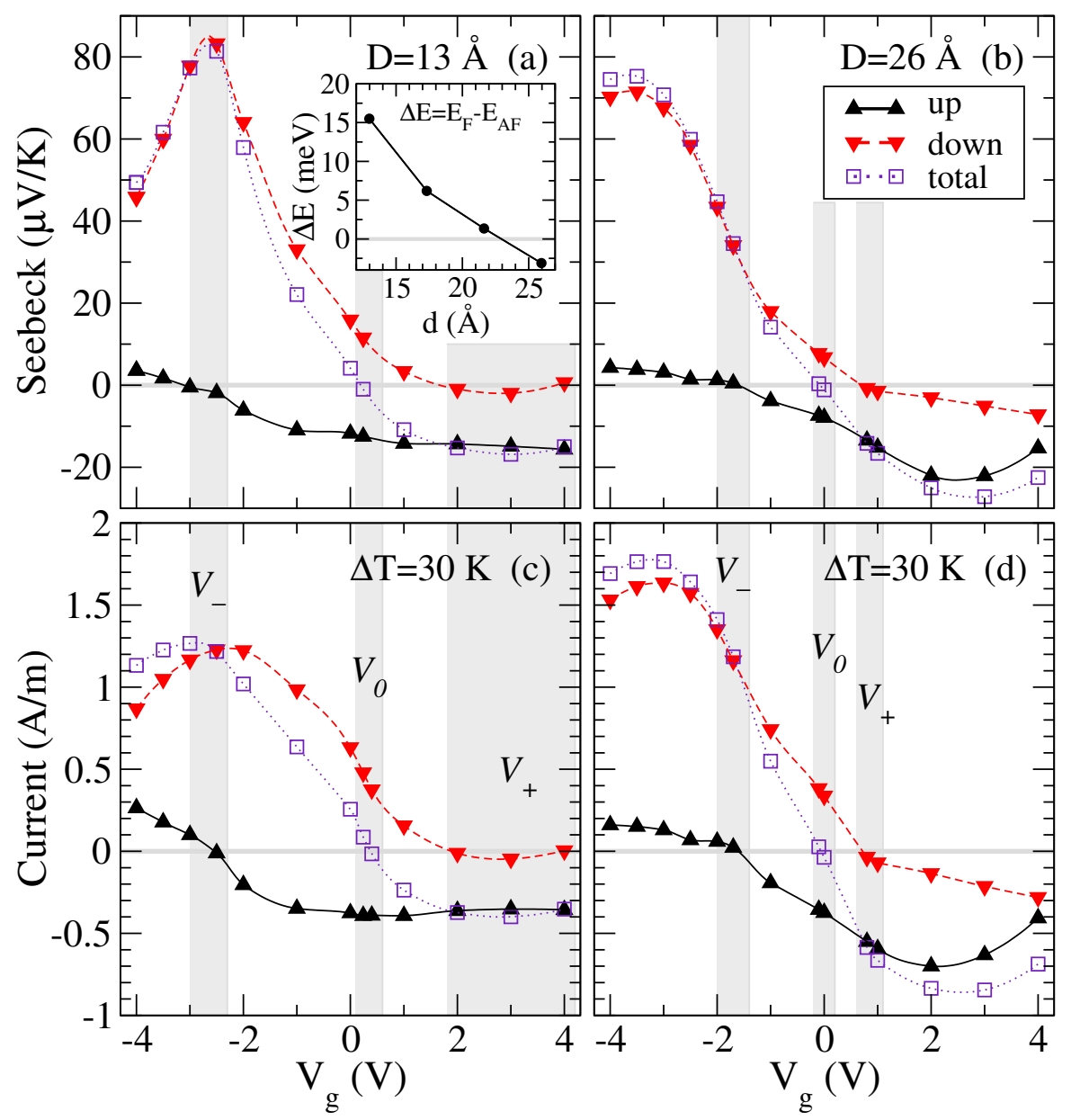

Figura 5.3: Coeficiente Seebeck em função do potencial de gate, $V_{g}$, para $D=13 \AA$ (a) e $D=26 \AA$ (b) à temperatura ambiente $\left(T_{L}=T_{R}=300 \mathrm{~K}\right)$. Corrente versus $V_{g}$ para $D=13 \AA$ (c) e $D=26 \AA$ (d) $\left(T_{L}=300 \mathrm{~K}\right.$ e $\left.T_{R}=330 \mathrm{~K}\right)$. O quadro em (a) mostra a diferença de energia total entre os alinhamentos $\mathrm{F}$ e AF em função da distância Mn-Mn, $d$. Os intervalos $V_{-}, V_{0}$ e $V_{+}$são discutidos no texto. As linhas são apenas guias para a visão.

em três comportamentos distintos:

1. $S_{\uparrow} \approx 0$ e $S_{\downarrow}>0$ (quando $V_{g} \approx V_{-}$);

2. $S_{\uparrow} \approx-S_{\downarrow}$ (quando $V_{g} \approx V_{0}$ );

3. $S_{\uparrow}<0$ e $S_{\downarrow} \approx 0$ (quando $V_{g} \approx V_{+}$).

A corrente (Eq. (5.1) $)$, que pode ser aproximada por $I_{\sigma}=-G_{\sigma} S_{\sigma} \Delta T$, onde $G_{\sigma}$ é a condutância, segue o mesmo comportamento. Quando $V_{g} \approx V_{-}(\mathbf{1}), I_{\uparrow} \approx 0 \mathrm{e}$ 


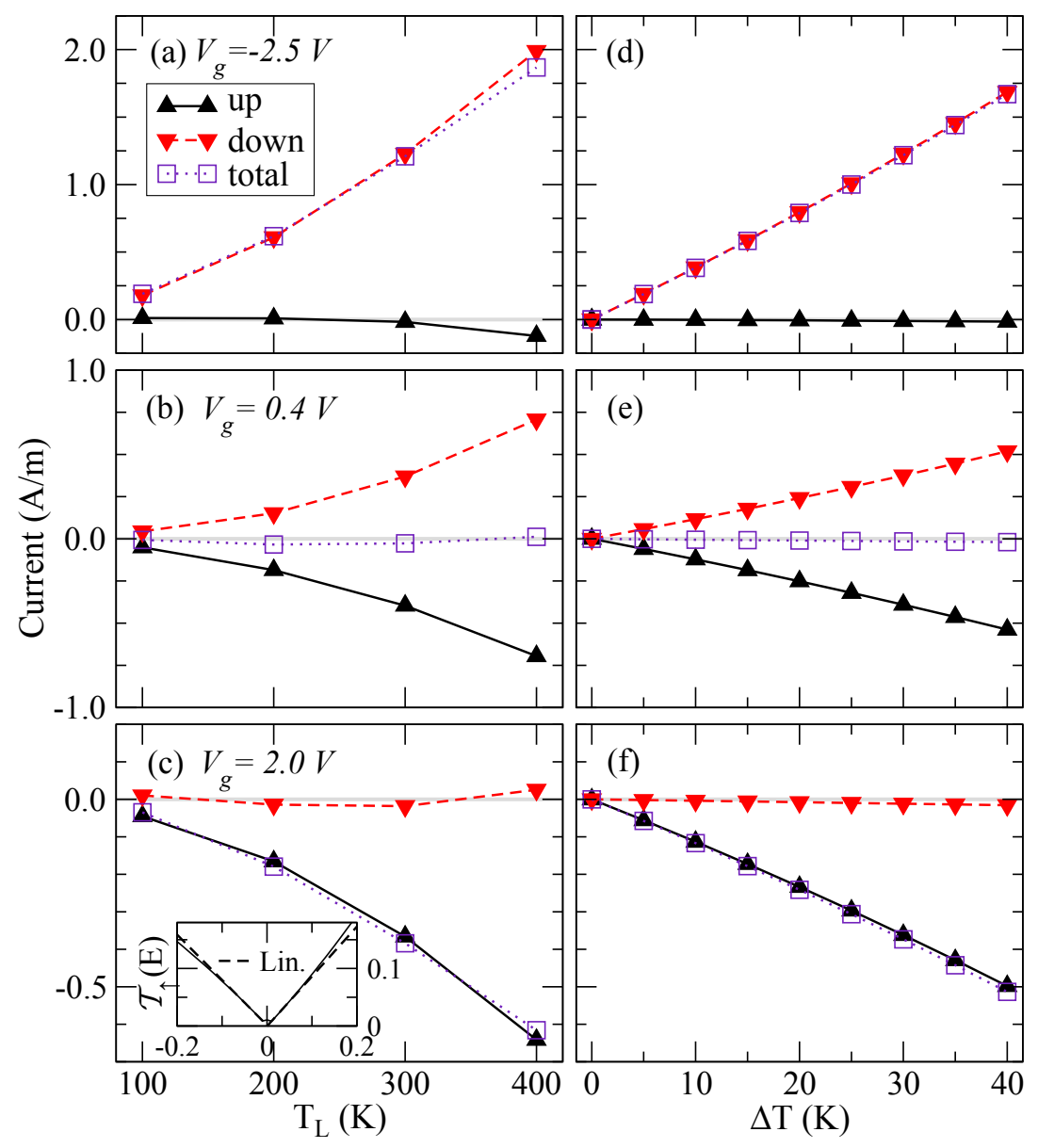

Figura 5.4: (a-c) Corrente em função da temperatura, $T_{L}$, para uma uma diferença de temperatura fixa de $30 \mathrm{~K}\left(T_{R}=T_{L}+30 \mathrm{~K}\right)$. (d-f) Corrente em função da diferença de temperatura entre os eletrodos, $\Delta T$, (o eletrodo esquerdo é mantido a temperatura ambiente, $T_{L}=300 \mathrm{~K}$, e $T_{R}=T_{L}+\Delta T$ ) para as voltagens de $V_{g}=-2.5$ $\mathrm{V}, 0.4 \mathrm{~V}$ e $2.0 \mathrm{~V}$. Note as diferenças de escala. As linhas são apenas guias para a visão.

há apenas $I_{\downarrow}$ fluindo; quando $V_{g} \approx V_{0}(\mathbf{2})$, o sistema apresenta correntes de spins contrários fluindo em direções opostas, sem transporte de carga líquido; e quando $V_{g} \approx V_{+}(\mathbf{3}), I_{\downarrow} \approx 0$ e há apenas corrente $I_{\uparrow}$ fluindo.

Para investiga como os comportamentos acima mencionados variam em função de variações na temperatura, calculamos a corrente para diferentes $T$ e $\Delta T$ para valores particulares de $V_{g}$ em cada um dos intervalos $V_{-}, V_{0}$ e $V_{+}$e $D=13 \AA$. Nas Figs. 5.4 a-c mostramos $I_{\sigma}$ em função da temperatura do eletrodo esquerdo, $T_{L}$, mantendo uma diferença de temperatura fixa de $\Delta T=30 \mathrm{~K}$ com o eletrodo direito 

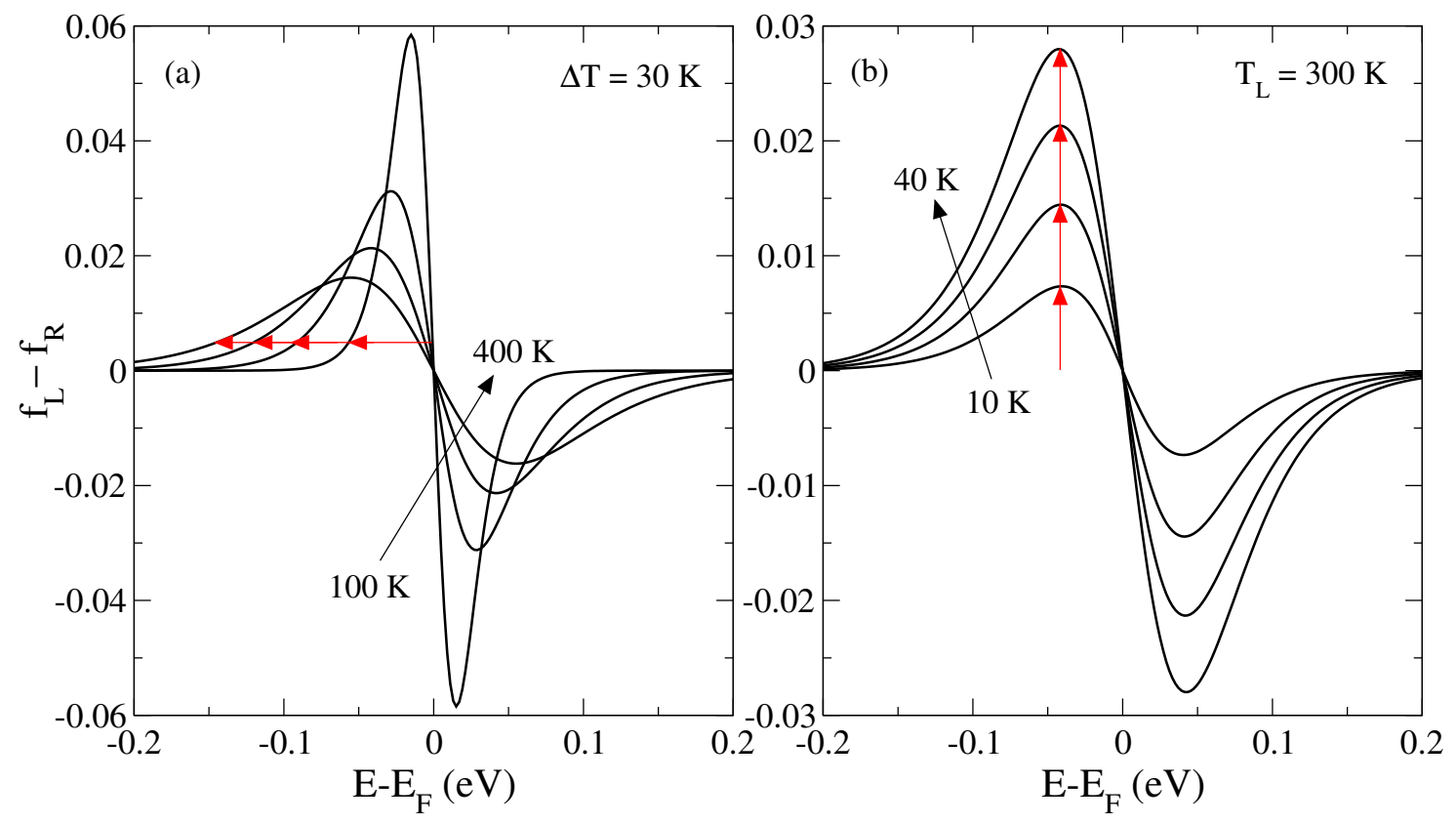

Figura 5.5: Diferença entre as distribuições de Fermi-Dirac entre os eletrodos esquerdo e direito, $f_{L}-f_{R}$, variando $T_{L}$ com $\Delta T=30 \mathrm{~K}$ fixo $\left(T_{R}=T_{L}+\Delta T\right)(\mathrm{a})$, e com $T_{L}=300 \mathrm{~K}$ fixo e variando $\Delta T$. As setas vermelhas em (a) e (b) indicam a variação na largura e amplitude de $f_{L}-f_{R}$, respectivamente.

; e nas Figs. $5.4 \mathrm{~d}-\mathrm{f}$, em função de $\Delta T$ mantendo $T_{L}=300 \mathrm{~K}$ fixo (a temperatura do eletrodo direito é sempre dada por $T_{R}=T_{L}+\Delta T$ ). O efeito de mudanças na temperatura sob $I_{\sigma}$ é governado principalmente pela diferença entre as ocupações dos contatos esquerdo e direito, dado por $f_{L}-f_{R}$ (veja a Eq. (5.1)).

Quando $T_{L}$ aumenta ( $\Delta T$ constante) a largura de $f_{L}-f_{R}$ aumenta (veja a Fig. 5.53 ), ou seja, os portadores de carga são termicamente excitados para energias mais altas (ou mais distantes de $E_{F}$ ), nas quais $\mathcal{T}_{\sigma}(E)$ se desvia da linearidade, resultando no comportamento não linear visto nas Figs. 5.4 a-c. No quadro da Fig. 5.4 c, mostramos em detalhe como $\mathcal{T}_{\uparrow}(E)$ desvia-se da linearidade (linha tracejada) a partir de aproximadamente $|E| \gtrsim 0.1 \mathrm{eV}$.

Por outro lado, quando $\Delta T$ varia ( $T_{L}$ constante) as energias para as quais os portadores de carga são termicamente excitados permanece constante, variando apenas sua quantidade, ou seja, a largura de $f_{L}-f_{R}$ permanece praticamente inalterada, 


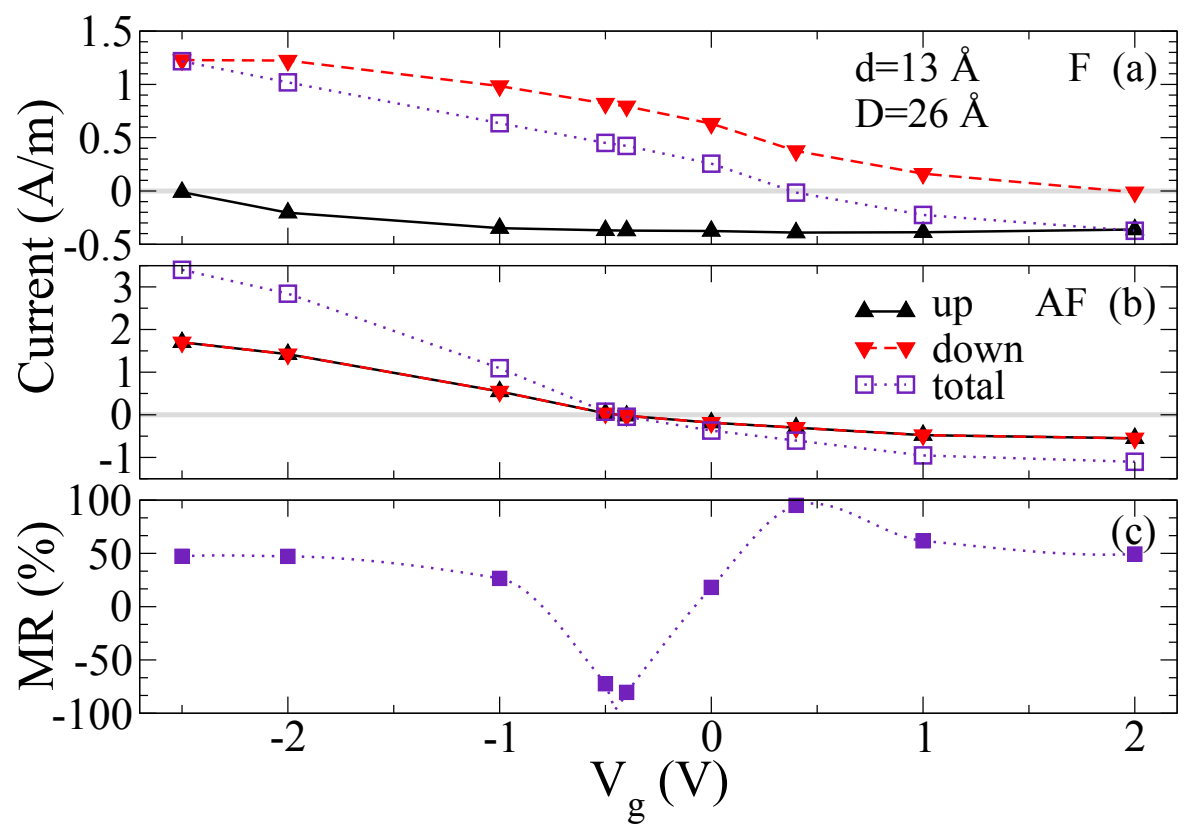

Figura 5.6: Corrente de uma supercélula com dois Mn@MV nos alinhamentos ferromagnético (a) e antiferromagnético (b) em função do potencial de gate, $V_{g}$. (c) Magnetorresistência termicamente induzida. As linhas são apenas guias para a visão.

mudando apenas sua amplitude, como mostrado na Fig. 5.5b. Assim, tem-se o comportamento $I_{\sigma} \propto \Delta T$ visto nas Figs. $5.4 \mathrm{~d}-\mathrm{f}$.

Para investigar como a ordem magnética entre os átomos de Mn influenciaria o sistema, calculamos as correntes para os alinhamentos F e AF, mostrados nas Figs. 5.6 a e $5.6 \mathrm{~b}$, respectivamente. No alinhamento F, a corrente exibe uma pronunciada polarização de spin, enquanto que no alinhamento AF não há polarização de spin, como esperado do espectro degenerado em spin neste caso.

A magnetorresistência termicamente induzida (MR) é dada por (em \%)

$$
M R=\frac{\left|I^{F}\right|^{-1}-\left|I^{A F}\right|^{-1}}{\left|I^{F}\right|^{-1}+\left|I^{A F}\right|^{-1}} \times 100
$$

e depende apenas nas correntes totais $I^{F(A F)}=I_{\uparrow}^{F(A F)}+I_{\downarrow}^{F(A F)}$. MRs colossais $( \pm 100 \%)$ são obtidas quando $I^{F}$ ou $I^{A F}$ se anulam. Ambas situações podem ser obtidas variando $V_{g}$, como pode ser visto na Fig. 5.6. Para $V_{g} \approx 0.4 \mathrm{~V}$, tem-se 
$I_{\uparrow}^{F}=-I_{\downarrow}^{F}$, o que resulta em uma corrente total nula para o alinhamento $\mathrm{F}, I^{F}=0$. Já para $V_{g} \approx-0.4 \mathrm{~V}$, obtém-se $I_{\uparrow}^{A F}=I_{\downarrow}^{A F}=0$, e obviamente, $I^{A F}=0$. Portanto, vemos que variando o potencial de gate nessa região $\left(-0.4 \mathrm{~V} \lesssim V_{g} \lesssim 0.4 \mathrm{~V}\right)$ é possível ajustar o valor da magnetorresistência em qualquer valor entre $-100 \%$ e $+100 \%$, como visto na Fig. 5.6.

Vamos agora discutir quais os desafios que a realização experimental deste dispositivo enfrentaria. Primeiramente é importante notar que a geometria do dispositivo proposto neste trabalho é bem específica. Apesar de ser possível replicar esta geometria experimentalmente com um microscópio de transmissão eletrônica (TEM Transmission Electron Microscope), no qual o feixe pode ser focalizado com resolução de até $1 \AA$ [101, 108], seria mais fácil, do ponto de vista experimental, dopar o grafeno por bombardeamento com íons. Neste caso, os Mn estariam aleatoriamente distribuídos sobre o grafeno e a predominância do tipo de defeitos criados poderia ser ajustada pela energia do feixe [109, 110, 104]. Sob estas condições, há indícios de que o efeito da desordem na distribuição dos defeitos pode levar o sistema a um regime localizado no qual a assimetria em spin das propriedades de transporte podem ser promovidas [111, 112]. Outro ponto a considerar é o uso de outros metais. Embora, como dito anteriormente, o Mn apresente uma estrutura eletrônica ótima (veja a fig. 5.1d), com níveis localizados com spins diferentes próximos ao nível de Fermi, outros metais também podem apresentar o mesmo efeito, como Fe, Ni ou Co [101, 74]. Em princípio, qualquer metal que tenha uma transmitância dependente de spin nas proximidades de $E_{F}$ poderá apresentar efeito Seebeck dependente de spin (veja a Eq. 5.2). Finalmente, o efeito causado pela presença de um substrato também deve ser levada em conta em uma situação experimental. Dependendo do substrato, o grafeno pode sofrer transferência de carga e apresentar uma dopagem tipo n ou p. Assim, as voltagens necessárias para atingir cada um dos três regimes podem ser diferentes. Dopagem local do substrato também poderia ser usada como substituição para o efeito do potencial de gate. 
Em suma, neste trabalho mostramos que grafeno dopado com Mn, com os $\mathrm{Mn}$ presos em monovacâncias, apresenta efeito Seebeck dependente de spin. Ademais, o dispositivo proposto pode ser ajustado através de um potencial de gate $V_{g}$ de forma a ter corrente de um determinado spin (com polarização de quase 100\%) fluindo em uma dada direção, ou correntes com cada spin fluindo em sentidos contrários e sem transporte de carga líquido. Vale ressaltar que neste trabalho, é proposto pela primeira vez o uso de grafeno 2D para caloritrônica de spin. 


\section{Capítulo 6}

\section{Junção molecular Au-BDT-Au}

Entender as propriedades físicas e químicas de junções moleculares é fundamental para o desenvolvimento da eletrônica molecular [113]. Em 1997, M. A. Reed et al [56] mediram pela primeira vez a condutância de uma única molécula de benzeno-1,4ditiol (BDT) 1 conectada a contatos de ouro, através da técnica de quebra de junção mecanicamente controlável (MCBJ - Mechanically Controllable Break Junction). Desde então, a molécula de BDT se tornou referência para o estudo de junções moleculares [57].

Recentemente, o estudo das propriedades de transporte eletrônica do BDT voltou a receber atenção. Particularmente, N. J. Tao et al. [114 mostraram experimentalmente que a condutância de uma única molécula de BDT conectada a contatos de ouro aumenta consideravelmente ao ser esticada, quando a junção formada entre os contatos e a molécula está perto do ponto de ruptura. Este aumento, mecanicamente induzido, foi atribuído à ressonância causada pelo realinhamento do HOMO (Highest Occupied Molecular Orbital), que se aproxima do nível de Fermi dos contatos a medida que o acoplamento molécula-metal é enfraquecido pelo estiramento da junção. Além disso, ficou demonstrada a possibilidade de construção de dispo-

\footnotetext{
${ }^{1}$ Uma molécula de benzeno com dois grupos $-\mathrm{S}-\mathrm{H}$ (tiol) ligados a carbonos em posições opostas do anel (posição para ou -1,4-).
} 
sitivos moleculares eletromecânicos, onde forças podem ser usadas para ajustar as propriedades eletrônicas, colocando o sistema em (ou fora de) ressonância.

No tocante às propriedades termoelétricas, a primeira medida da termopotência ou coeficiente Seebeck, $S$, de uma junção molecular foi realizada em 2007 por A. Majumdar et al. [13] usando MCBJs controladas por um microscópio de tunelamento (STM-MCBJ - Scanning Tunneling Microscope - Mechanically Controllable Break Junction) 2. Embora o coeficiente Sebeeck medido tenha sido pequeno, de $S=8.7 \pm 2.1 \mu \mathrm{V} / \mathrm{K}$ para o BDT e de $S=14.2 \pm 3.2 \mu \mathrm{V} / \mathrm{K}$ para o TBDT (Tri-BDT, cadeia de três anéis de benzeno), este trabalho pioneiro definiu que o transporte em junções de BDT é mediado por buracos (através do HOMO, tipo $p$ ), mostrando que medidas de $S$ servem para definir a natureza do transporte eletrônico, e fomentou o interesse pelo estudo das propriedades termoelétricas em dispositivos moleculares [55, 115, 116, 67, 117, 118, 119]. Mais recentemente, em 2011, L. Venkataraman et al. [119] realizaram pela primeira vez medidas simultâneas da condutância e do coeficiente Seebeck em junções moleculares.

Como as propriedades de transporte de carga dependem de como os orbitais de fronteira estão posicionados em relação ao nível de Fermi [114] e, atualmente, é possível fazer medidas concomitantes de propriedades termoelétricas e de transporte [119, 120], surge a pergunta de como as propriedades termoelétricas de uma junção molecular variam em função de seu estiramento. Neste trabalhq3 estudamos esta questão e mostramos que as propriedades termoelétricas de uma junção molecular com uma única molécula de BDT podem ser ajustadas através de alongamento mecânico. Também propomos um esquema para otimizar a figura de mérito de junções moleculares em geral.

Os resultados para a evolução estrutural da junção Au-BDT-Au foram baseados em cálculos de energia total de DFT (veja o apêndice A) usando o código

\footnotetext{
${ }^{2}$ Nesta técnica, a ponta do STM é aproximada e afastada repetidas vezes de uma superfície na qual a molécula de interesse foi depositada, formando e rompendo a junção durante o processo.

${ }^{3}$ Realizado em colaboração com o Prof. Dr. Renato Borges Pontes.
} 
SIESTA [70, 71]. Nas contas, usamos a aproximação de gradiente generalizado de Perdew-Burke-Ernzerhof (PBE-GGA) [106] para o funcional de troca e correlação, pseudopotenciais de norma conservada [75], uma base duplo- $\zeta$ polarizada (DZP) e um grid definido por uma energia de corte (mesh cutoff) de 240 Ry. A zona de Brillouin foi amostrada com $4 \times 4 \times 1$ pontos $k$ no esquema de Monkhorst-Pack [77. As geometrias consistem de uma molécula de BDT conectada a dois slabs de ouro, modelados como uma superfície de Au $3 \times 3$ com cinco camadas na direção [111]. Em todos os cálculos as posições dos átomos da molécula de BDT e das duas primeiras camadas de $\mathrm{Au}$ adjacentes ao BDT foram relaxadas até atingir uma força menor que $0.02 \mathrm{eV} / \AA$, enquanto que as outras três camadas foram mantidas fixas na posição de bulk. Para simular o estiramento a separação entre os eletrodos (as superfícies de $\mathrm{Au}$ ) foi aumentada gradativamente em passos de $0.5-1.4 \AA$, sendo relaxada para cada novo comprimento.

Os cálculos de transporte foram realizados com o código TRANSAMPA [14, 15] que aplica o formalismo de funções de Green fora do equilíbrio acoplado com DFT $(\mathrm{NEGF}+\mathrm{DFT})$. Correções de auto-interação (SIC - Self Interaction Correction) foram incluídas segundo o esquema proposto por Filippetti e Spaldin [121, 122] conforme implementado no TRANSAMPA. A inclusão deste tipo de correção altera drasticamente, para melhor, as propriedades de transporte neste tipo de junções [57, 123, 124]. Para os cálculos de transporte foram empregados $2 \times 2 \times 1$ pontos $k$ para amostrar a zona de Brillouin.

No formalismo de Landauer-Büttiker as condutância eletrônica e térmica eletrônica, $G$ e $K_{e l}$ respectivamente, e o coeficiente Seebeck podem ser escritos como

$$
\begin{aligned}
G & =\frac{e^{2}}{h} \mathcal{K}_{0} \\
K_{e l} & =\frac{1}{h \bar{T}}\left(\mathcal{K}_{2}-\frac{\mathcal{K}_{1}^{2}}{\mathcal{K}_{0}}\right) \\
S & =-\frac{1}{e \bar{T}} \frac{\mathcal{K}_{1}}{\mathcal{K}_{0}},
\end{aligned}
$$


onde $e$ é a carga do elétron, $h$ é a constante de Planck, $\bar{T}$ é a temperatura média da junção molecular e $\mathcal{K}_{n}$ é definido como

$$
\mathcal{K}_{n}=\int \mathcal{T}(E)\left(-\frac{\partial f}{\partial E}\right)(E-\mu)^{n} d E
$$

onde $\mathcal{T}(E)$ é a função de transmitância, $f$ é a função de distribuição de Fermi-Dirac e $\mu$ é o potencial químico.

A figura de mérito termoelétrica é definida como

$$
Z T=\frac{S^{2} G}{K} T=\frac{S^{2} G}{K_{e l}+K_{p h}} T
$$

onde $K$ é condutância térmica total, composta pelas contribuições eletrônica, $K_{e l}$, e fonônica, $K_{p h}$. Note que ignorando a contribuição oriunda das vibrações dos átomos podemos escrever um limite superior para $Z T$ que depende apenas de grandezas eletrônicas,

$$
Z T_{e l}=\frac{S^{2} G}{K_{e l}} T
$$

Daqui em diante abandonaremos o subscrito el e nos referiremos apenas à parte eletrônica de figura de mérito. Inserindo as Eqs. (6.1)- 6.3 na Eq. 6.6 podemos reescrever a expressão para a figura de mérito como

$$
Z T=\left(\frac{\mathcal{K}_{2} \mathcal{K}_{0}}{\mathcal{K}_{1}^{2}}-1\right)^{-1}
$$

Na Fig. 6.1 mostramos nove geometrias representando diferentes estágios de estiramento de uma junção Au-BDT-Au em um experimento STM-MCBJ. Na configuração inicial (Fig. 6.1 a) a separação entre os eletrodos de $L=5.50 \AA$ e a molécula está paralela à superfície de Au. A medida que a separação entre os contatos aumenta de $L=6.45 \AA$ até $L=11.35 \AA$ (Figs. 6.1p-f) a molécula vai se inclinando cada vez mais até ficar conectada por apenas um átomo de Au em cada 

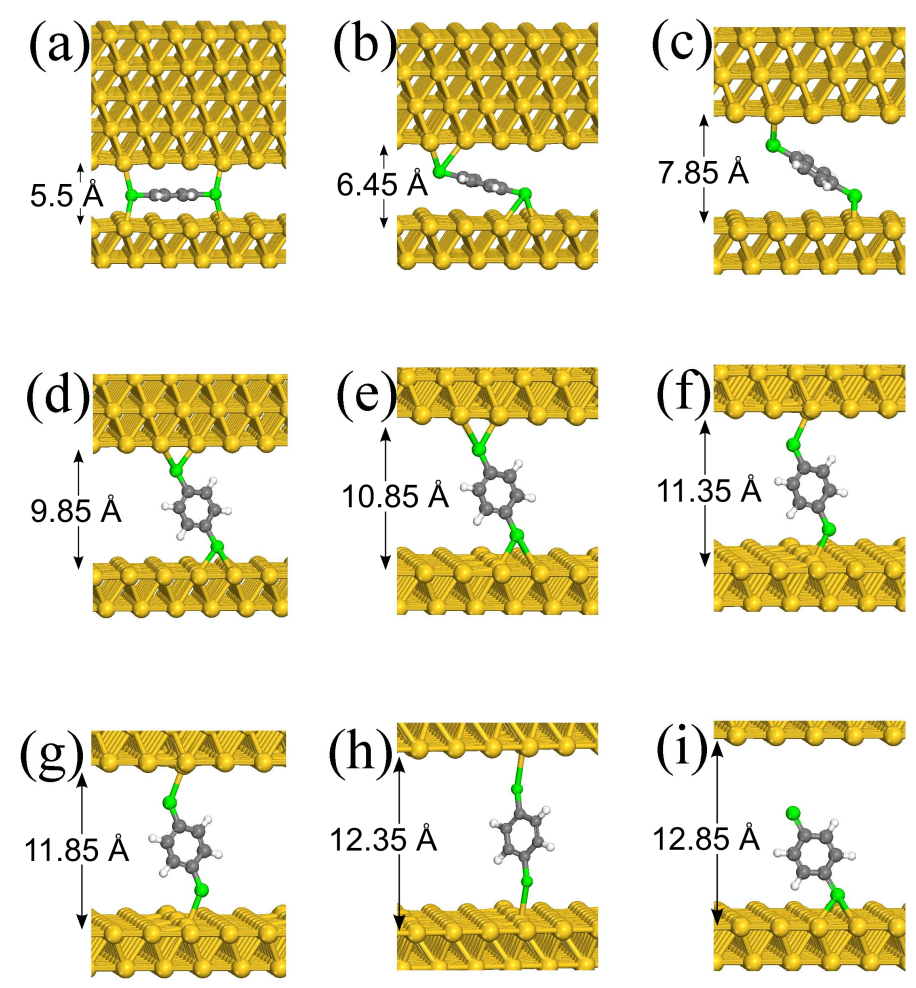

Figura 6.1: Visão esquemática das geometrias otimizadas para diversos comprimentos de estiramento.

superfície. Com estiramentos maiores, a molécula de BDT fica em uma configuração mais vertical (Figs. 6.1p-h) até que a ligação Au-S em uma das superfícies se rompa quando o estiramento é de $L=12.85 \AA$ (Figs. 6.1).

Na Fig. 6.2 a-i apresentamos as funções de transmitância, $\mathcal{T}(E)$, obtidas para as configurações correspondentes às mostradas na Fig. 6.17-i e na Fig. 6.2j, a condutância, $G$, calculada segundo a Eq. 6.1 para temperatura ambiente $(T=$ 300 K), em função do estiramento da junção, $L$. Note que para a menor distância de separação, $L=5.50 \AA$ (Fig. 6.2 a), obtemos o valor mais alto pra a condutância, $G_{L=5.50 \AA}=1.36 G_{0}$. Isto pode ser explicado pela configuração paralela da molécula com as superfícies de Au, propiciando que a interação dos orbitais $p_{z}$ do BDT com os contatos criem mais canais de transmissão. Assim que a junção vai sendo esticada, a condutância cai rapidamente para $G_{L=6.45 \AA}=0.20 G_{0}$ e $G_{L=7.85 \AA}=0.07 G_{0}$. Para separações a partir de $L=7.85 \AA$ até $L=11.35 \AA$ a condutância da junção varia 

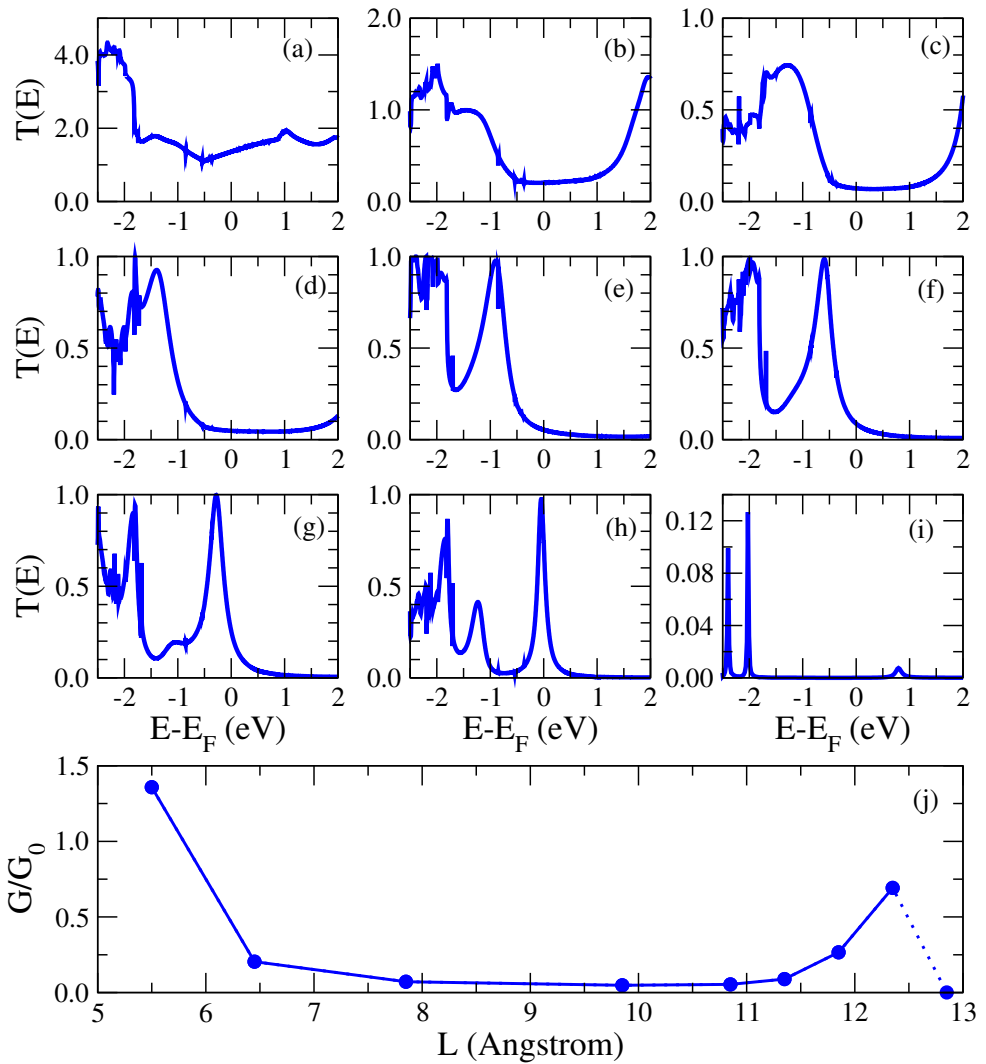

Figura 6.2: (a-i) Transmitância em função da energia, $\mathcal{T}(E)$, de uma junção AuBDT-Au para vários estiramentos. (j) Condutância em função do estiramento.

muito pouco em função de seu alongamento, $G_{L=9.85 \AA}=0.05 G_{0}, G_{L=10.85 \AA}=0.05 G_{0}$ e $G_{L=11.35 \AA}=0.09 G_{0}$, sendo determinada pelo acoplamento das extremidades do BDT com as superfícies de ouro. Esticando mais o sistema, há um aumento súbito de $G$, chegando a $0.7 G_{0}$ em $L=12.35 \AA$, antes da ligação Au-S se romper em $L=12.85 \AA$, quando a junção é quebrada e a condutância despenca para $4 \times 10^{-4} G_{0}$. Este aumento da condutância se deve ao realinhamento do HOMO da molécula, provocando uma ressonância na função de transmissão que se aproxima do nível de Fermi a medida que a junção é esticada (veja as Figs. 6.2 2 -h) [57].

As termopotências, calculadas a partir de $\mathcal{T}(E)$ segundo a Eq. (6.3), são apresentadas na Figura 6.3 em função da energia, $S(E)$, (Figs. 6.2 a-j) para as transmitâncias correspondentes mostradas nas Figs. $6.2 \mathrm{a}-\mathrm{j}$, e no nível de Fermi, $S\left(E_{F}\right)$, em função da separação dos contatos da junção para as temperaturas de $300 \mathrm{~K}$ e $10 \mathrm{~K}$. 


\begin{tabular}{c|c|c|l}
$S(\mu \mathrm{V} / \mathrm{K})$ & $G\left(G_{0}\right)$ & $E_{F}-E_{H O M O}(\mathrm{eV})$ & Referência \\
\hline $8.7 \pm 2.1$ & $\sim 0.1$ & $1.2^{a}$ & {$[13$} \\
$7.2 \pm 0.2$ & $0.011^{a}$ & $1.16 \pm 0.02^{a}$ & {$[115$} \\
12.4 & 0.05 & 0.90 & ${ }^{b} L=10.85 \AA$ \\
7.2 & 0.05 & & ${ }^{b} L=10.45 \AA$ (estimativa)
\end{tabular}

Tabela 6.1: Medidas do coeficiente Seebeck de uma junção Au-BDT-Au a temperatura ambiente. ${ }^{a}$ Valor ajustado por um modelo teórico. ${ }^{b}$ Este trabalho.

Note que para ambas as temperaturas, o coeficiente Seebeck é praticamente nulo até uma separação de aproximadamente $\sim 10 \AA$ (variando de -2.5 até $+2.5 \mu \mathrm{V} / \mathrm{K}$ para $300 \mathrm{~K}$ e de -0.08 até $+0.08 \mu \mathrm{V} / \mathrm{K}$ para $10 \mathrm{~K})$. A partir dessa separação, $S\left(E_{F}\right)$ começa a aumentar, atingindo os valores máximos de $40 \mu \mathrm{V} / \mathrm{K}$ e $2.1 \mu \mathrm{V} / \mathrm{K}$ nas proximidades do ponto de ruptura da junção nas temperaturas de $300 \mathrm{~K}$ e $10 \mathrm{~K}$, respectivamente. Este notável aumento em função do estiramento é devido ao deslocamento das oscilações de $S(E)$ (os picos negativo e positivo, claramente visíveis nas Figs. 6.3 $(-\mathrm{i})$ associadas à ressonância em $\mathcal{T}(E)$ correspondente ao HOMO da molécula.

Em dois trabalhos recentes, A. Majumdar e R. A. Segalman et al. [13, 115] reportaram medidas do coeficiente Seebeck de junções Au-BDT-Au em temperatura ambiente, de $8.7 \pm 2.1 \mu \mathrm{V} / \mathrm{K}$ e $7.2 \pm 0.2 \mu \mathrm{V} / \mathrm{K}$ respectivamente (veja a Tabela 6.1). Em nossos cálculos, encontramos o valor de $S=12.4 \mu \mathrm{V} / \mathrm{K}$ para um comprimento de separação dos eletrodos de $L=10.85 \AA$. Fazendo uma interpolação (spline cúbica de Akima) entre os valores calculados de $S\left(E_{F}\right)$ em função de $L$, podemos estimar que o estiramento da junção para obter $S=7.2 \mu \mathrm{V} / \mathrm{K}$ é de $10.45 \AA$. Os valores calculados para a condutância e para a posição do HOMO em relação ao nível de Fermi concordam qualitativamente com os resultados reportados nestes dois experimentos e cálculos anteriores na literatura [57, 123, 124].

Na Figura 6.4 apresentamos os valores calculados para a figura de mérito, $Z T$ (Eq. 6.7)), em função da energia (Figs. 6.4A-i) para uma temperatura de $300 \mathrm{~K}$, e no nível de Fermi em função do estiramento da junção para as temperaturas de 

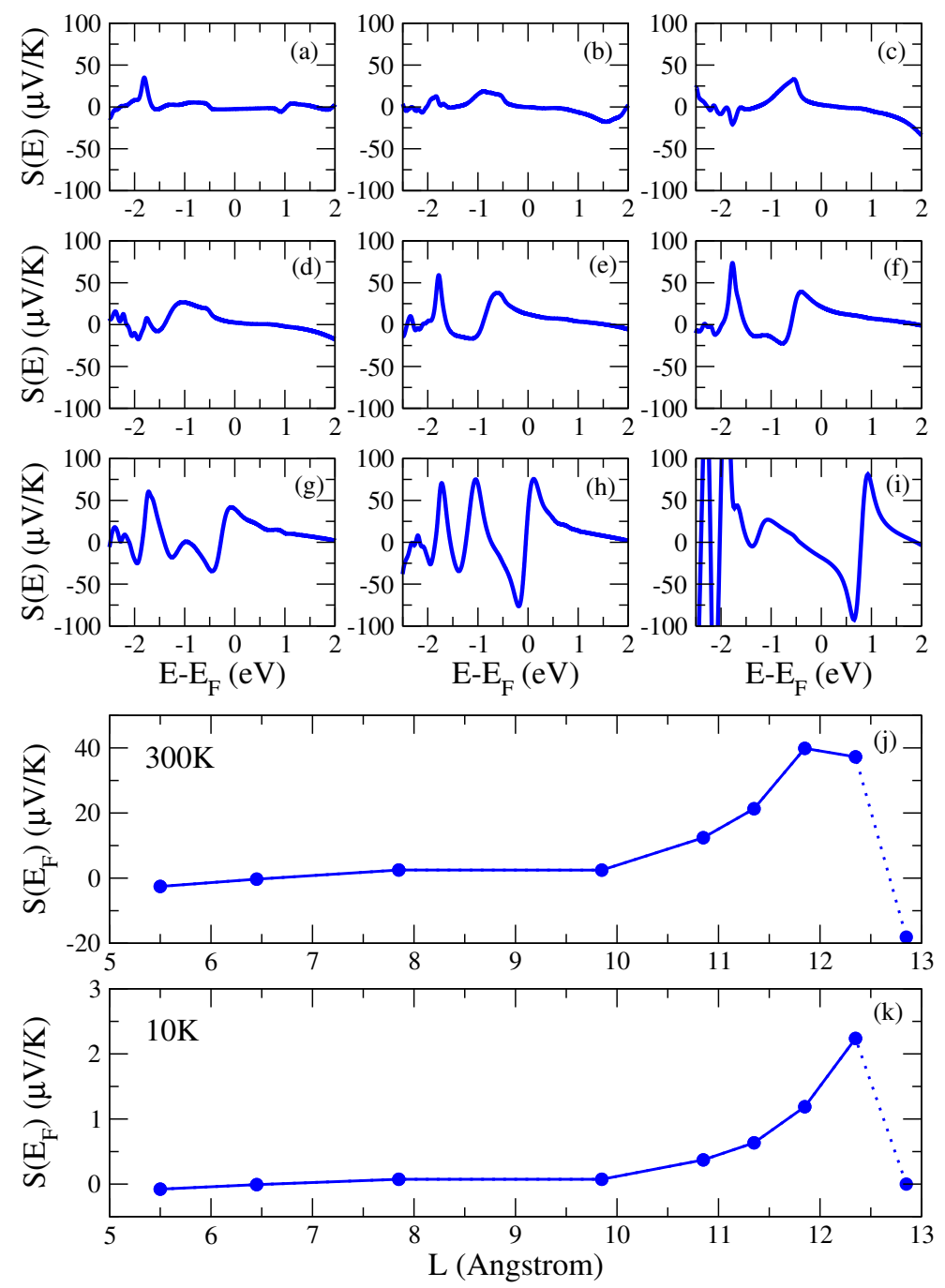

Figura 6.3: (a-i) Coeficiente Seebeck de uma junção Au-BDT-Au em função da energia, $S(E)$, para vários estiramentos e no nível de Fermi, $S\left(E_{F}\right)$, em função do estiramento, $L$, para as temperaturas de (j) $300 \mathrm{~K}$ e (k) $10 \mathrm{~K}$. 

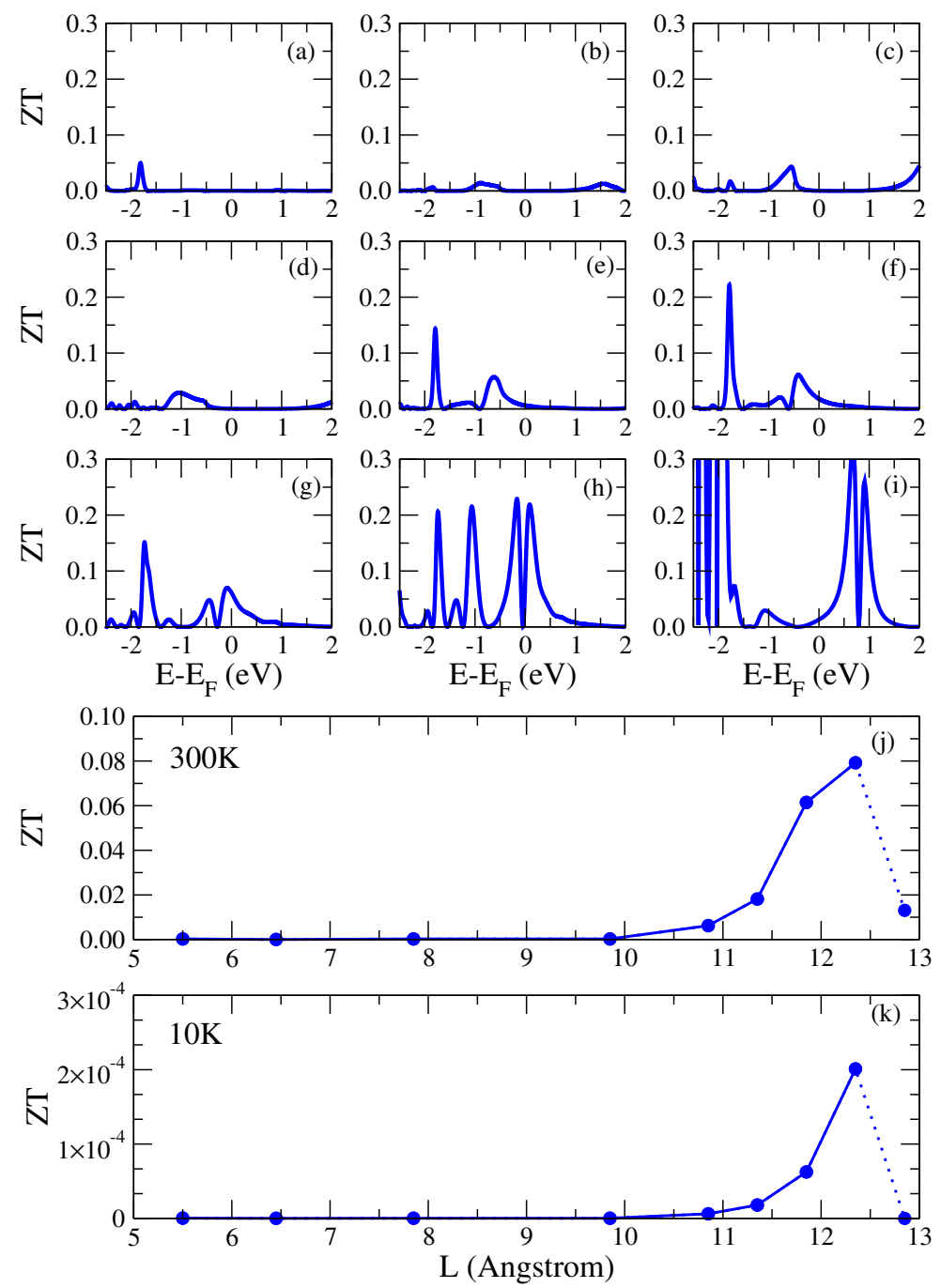

Figura 6.4: (a-i) Figura de mérito adimensional, $Z T$, de uma junção Au-BDT-Au em função da energia para vários estiramentos e no nível de Fermi, $Z T\left(E_{F}\right)$, em função do estiramento para as temperaturas de (j) $300 \mathrm{~K}$ e (k) $10 \mathrm{~K}$. 
$300 \mathrm{~K}$ e $10 \mathrm{~K}$ (Figs. 6.4 -k). De forma semelhante ao coeficiente Seebeck (lembre que $Z T \propto S^{2}$, Eq. (6.6), a figura de mérito é praticamente nula até o comprimento de separação de $L=10.85 \AA$. A partir de $L=10.85 \AA$ o duplo pico em $Z T(E)$ associado à ressonância do HOMO cruza o nível de Fermi (Figs. 6.4 -i). Assim, o valor de $Z T$ no nível de Fermi apresenta um aumento súbito, atingindo os máximos de $Z T_{300 \mathrm{~K}}=0.08$ e $Z T_{10 \mathrm{~K}}=2 \times 10^{-4}$ para um estiramento de $L=12.35 \AA$, retornando para valores quase nulos quando a junção é quebrada. Note que apesar do aumento na figura de mérito em função do estiramento, os valores máximos de $Z T$ alcançados são muito pequenos (em geral, deseja-se $Z T \gtrsim 1$, veja a seção 2.3.4, de forma que, como dispositivo termoelétrico, esta junção seria ineficiente. Apesar disso, há evidências experimentais e teóricas de que o aumento no número de anéis aromáticos na molécula resulta no aumento do coeficiente Seebeck [120, 118, 117, muito possivelmente resultando em um aumento de $Z T$. Também observamos que o comportamento observado de aumento da figura de mérito, o coeficiente Seebeck e a condutância, com o estiramento é devida à posição dos orbitais de fronteira (no caso do BDT, o HOMO) em relação ao nível de Fermi. Portanto, este mecanismo de ajuste das propriedades termoelétricas deve ser válido para junções moleculares em geral e, se um controle mais refinado do elongamento da junção for possível, pode-se buscar a configuração de maior $Z T$.

\subsection{Otimizando $Z T$ em junções moleculares.}

Vamos agora nos voltar para uma análise de como maximizar a figura de mérito termoelétrica de junções moleculares em geral mecanicamente. Otimizar $Z T$ é sabidamente um problema complicado dada a interdependência entre seus componentes: a condutância $G$, o coeficiente Seebeck $S$ e a condutância térmica eletrônica $K$. Por outro lado, como vimos anteriormente, no formalismo de Landauer-Büttiker $Z T$ depende apenas da função de transmitância, $\mathcal{T}(E)$, e da função de Fermi-Dirac através 
dos termos $\mathcal{K}_{n}$ (veja as Eqs. 6.4 e 6.7)).

A função de Fermi-Dirac, através do negativo de sua derivada, $-\partial f / \partial E$, define a janela, ou intervalo, de energias relevantes para os processos de transporte eletrônico (no regime linear), definindo um peso para cada valor de $E$. Este peso é máximo para $E=E_{F}$ e cai rapidamente para energias longe do nível de Fermi, sendo apreciável apenas num intervalo de alguns poucos $k_{B} T$, onde $k_{B}$ é a constante de Boltzmann, em torno de $E_{F}$. Já a função de transmitância, $\mathcal{T}(E)$, traz informação a respeito da estrutura eletrônica do sistema, definida por sua geometria e composição específica, no caso aqui estudado, a junção molecular Au-BDT-Au.

De um ponto de vista estatístico, a probabilidade de um elétron em um dos eletrodos alcançar o outro, seja através de uma diferença de potencial ou temperatura, é governada pela convolução de duas densidades de probabilidade, explicitamente $\mathcal{T}(E)$ e $-\partial f / \partial E$. Vemos então, que com uma boa descrição da transmitância nas proximidades de $E_{F}$, na janela definida pela função de Fermi-Dirac, poderemos calcular a figura de mérito corretamente.

Os picos na transmitância associados ao HOMO do BDT para estiramentos maiores que $9 \AA$, mostrados nas Figs. $6.2 \mathrm{~d}$-i, apresentam um formato lorentziano,

$$
\mathcal{L}(E)=\frac{A}{1+\left(\frac{E-E_{0}}{\gamma}\right)^{2}},
$$

onde $A$ é a amplitude máxima, $E_{0}$ é a posição (em energia) do pico e $\gamma$ define sua largura. Na Figura 6.5 apresentamos os ajustes lorentzianos para os casos específicos com separação $L=11.85 \AA, L=12.35 \AA$ e $L=12.85 \AA$ (Figs. 6.5 2 -c, respectivamente), mostrando uma concordância muito boa com as curvas calculadas. Na Fig. 6.5 d apresentamos um teste mais rigoroso, comparando os valores obtidos para $Z T$ no nível de Fermi em função do estiramento através das transmitâncias ajustadas por lorenztianas com os calculados via método ab initio. Os resultados virtualmente idênticos nos dos casos, confirmam a validade do ajuste lorentziano das ressonâncias 

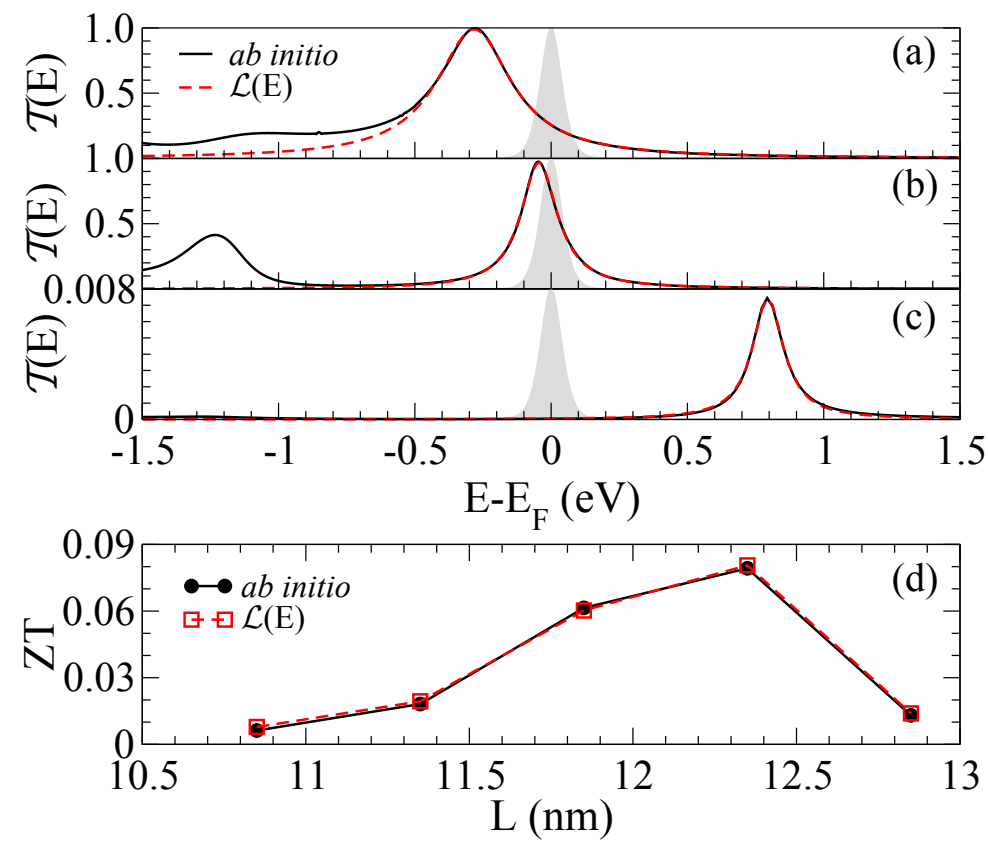

Figura 6.5: Transmitâncias para os estiramentos de (a) $L=11.85 \AA$, (b) $L=$ $12.35 \AA$ e (c) $L=12.85 \AA$. As linhas tracejadas vermelhas são funções lorentzianas, $\mathcal{L}(E)$, ajustando os valores calculados nos intervalos $[-0.5,0.5] \mathrm{eV}$ em (a-b) e $[-1.5,1.5] \mathrm{eV}$ em $(\mathrm{c})$. As áreas cinzas representam $-\partial f_{300 \mathrm{~K}} / \partial E$ em unidades arbitrárias. (d) Comparação das figuras de mérito, $Z T$, obtidas a partir das transmitâncias calculadas via método ab initio (NEGF + DFT) e dos ajustes lorentzianos .

em $\mathcal{T}(E)$. Assim, passaremos a modelar as transmitâncias de nosso sistema como funções lorentzianas. Esta abordagem, além de ser comum para explicar dados experimentais [13, 115, 120, tem a vantagem de ser simples e geral, de forma que pode ser aplicada a outras junções moleculares.

Inserindo a Eq. (6.8) na Eq. (6.7) é fácil ver que a amplitude $A$ se cancela na razão $\mathcal{K}_{2} \mathcal{K}_{0} / \mathcal{K}_{1}^{2}$. Conseqüentemente, o valor absoluto da transmitância não tem influência alguma na figura de mérito, isto é, apesar da expressão $Z T=S^{2} G / K$ sugerir o contrário o valor absoluto da condutância não influencia $Z T$, sendo este determinado apenas pela posição do pico em relação ao nível de Fermi, $E_{0}$, ou seja, a energia da ressonância (do HOMO, no nosso caso); e de sua largura, $\gamma$.

Na Figura 6.6 mostramos os valores calculados para a figura de mérito no nível de Fermi, $Z T\left(E_{F}\right)$, de transmitâncias de formato lorentziano em função de sua 

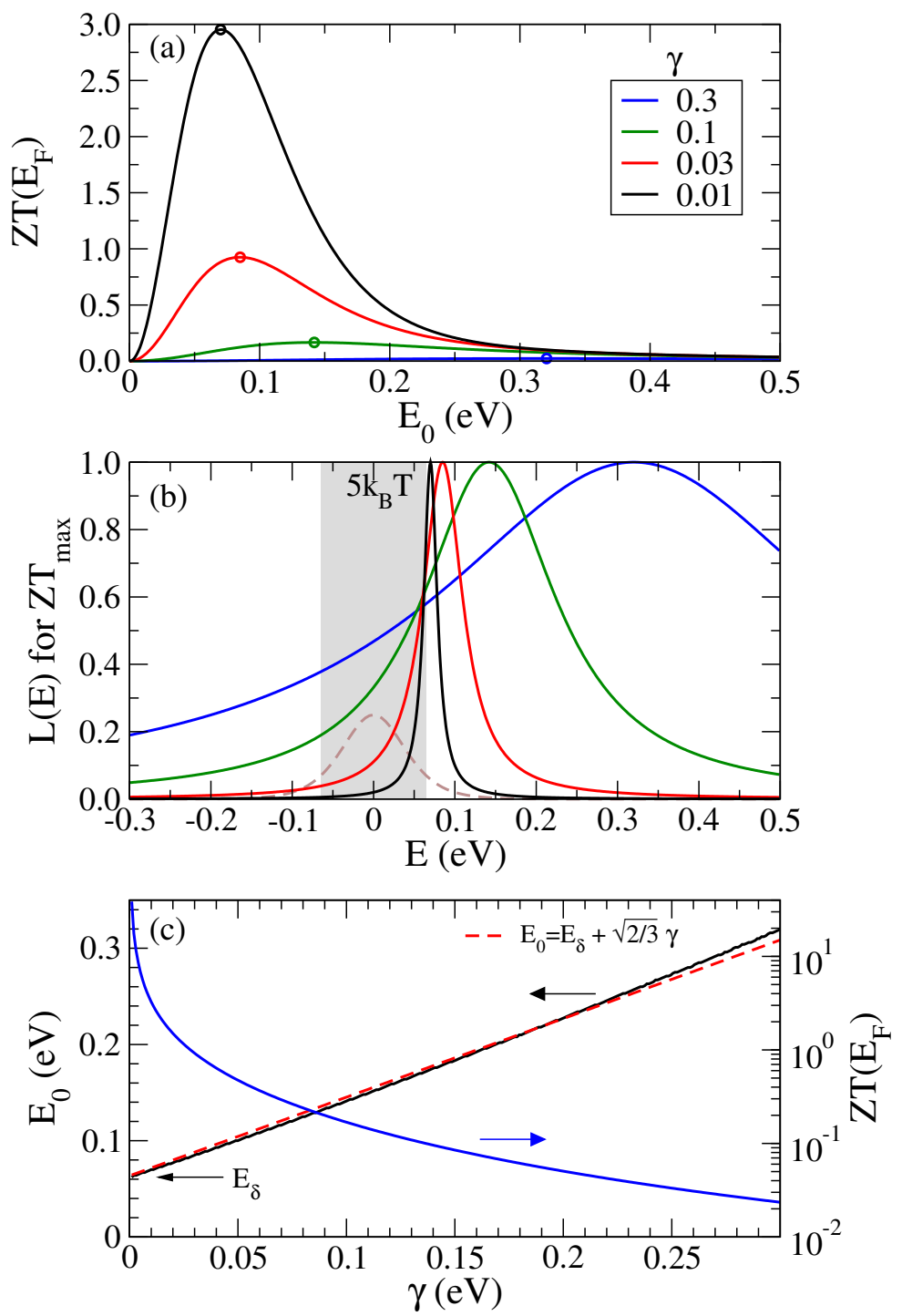

Figura 6.6: (a) Figura de mérito no nível de Fermi, $Z T\left(E_{F}\right)$, para transmitâncias de formato lorentziano com diversas larguras $\gamma$ em função da energia central da lorentziana, $E_{0}$. (b) Transmitâncias de formato lorentziano, $\mathcal{L}(E)$, para os valores de $E_{0}$ que rendem $Z T$ máximo no gráfico superior, marcados por círculos abertos em (a). A linha tracejada representa $-\partial f_{300 \mathrm{~K}} / \partial E$ em unidades arbitrárias. (c) Energia central (posição), $E_{0}$, que $\mathcal{L}(E)$ deve ter para render um $Z T$ máximo no nível de Fermi em função da largura $\gamma$. Como $\mathcal{L}(E)$ é uma função simétrica em torno de $E_{0}$, plotamos apenas valores para $E_{0}>0$. Em todos os casos, usamos um amplitude de $A=1$. 
posição $E_{0}$, para várias larguras $\gamma$. Como se pode ver no gráfico, as maiores figuras de mérito são obtidas para as lorentzianas mais estreitas (menor $\gamma$ ). Isto ocorre porque o termo $\mathcal{K}_{1}$, originário do coeficiente Seebeck (veja a Eq. 6.3p) e dado por

$$
\mathcal{K}_{1}=\int \mathcal{T}(E)\left(-\frac{\partial f}{\partial E}\right)\left(E-E_{F}\right) d E
$$

é uma medida da assimetria local da transmitância, que é maior, nas proximidades do pico, quanto mais estreita $\mathcal{L}(E)$ for. Este termo maximiza $Z T$ quanto ele é mais alto. As lorentzianas que correspondem às máximas figuras de mérito (marcadas com círculos na Fig. 6.6 a) são apresentadas na Figura 6.6p, onde podemos ver que as posições dos picos, $E_{0}$, vão se aproximando do nível de Fermi, a menos de um deslocamento de aproximadamente $2.5 k_{B} T$, a medida que a largura da lorentziana diminui. Este deslocamento é aquele que maximiza a assimetria de $\mathcal{T}(E)$ em torno do nível de Fermi. Procedendo desta maneira, é possível fazer uma varredura nos parâmetros que definem $\mathcal{L}(E)$, explicitamente, $E_{0}$ e $\gamma$; e buscar que posição (energia da ressonância) a lorentziana deve ter, dada sua largura, para render uma figura de mérito máxima. Os resultados desta varredura são mostrados na Fig. 6.66, onde mostramos qual $E_{0}$ rende $Z T\left(E_{F}\right)$ dada uma largura $\gamma$ para a transmitância. Veja que, teoricamente, a melhor transmitância possível ocorre no limite $\gamma \rightarrow 0$, quando a lorentziana tende a uma função Delta de Dirac, $\mathcal{L}\left(E ; E_{0}, \gamma\right) \rightarrow \delta\left(E-E_{0}\right)$, posicionada em uma energia de $E_{\delta}=63.6 \mathrm{meV}\left(2.46 k_{B} T\right)$ em relação ao nível de Fermi; onde $Z T$ tende ao infinito. Para larguras maiores que zero, a posição ótima da lorentziana aumenta ligeiramente em função de $\gamma$, enquanto que a figura de mérito máxima cai drasticamente.

Na Figura 6.7 apresentamos um mapa de cor representando todos os valores calculados de $Z T\left(E_{F}\right)$ em função da largura e posição de $\mathcal{L}(E)$, usados para encontrar o conjunto de posições $E_{0}$ que maximizam $Z T$ para uma dada largura, mostrados inicialmente na Fig. 6.6. e por uma linha branca pontilhada na Fig. 6.7 e os pon- 


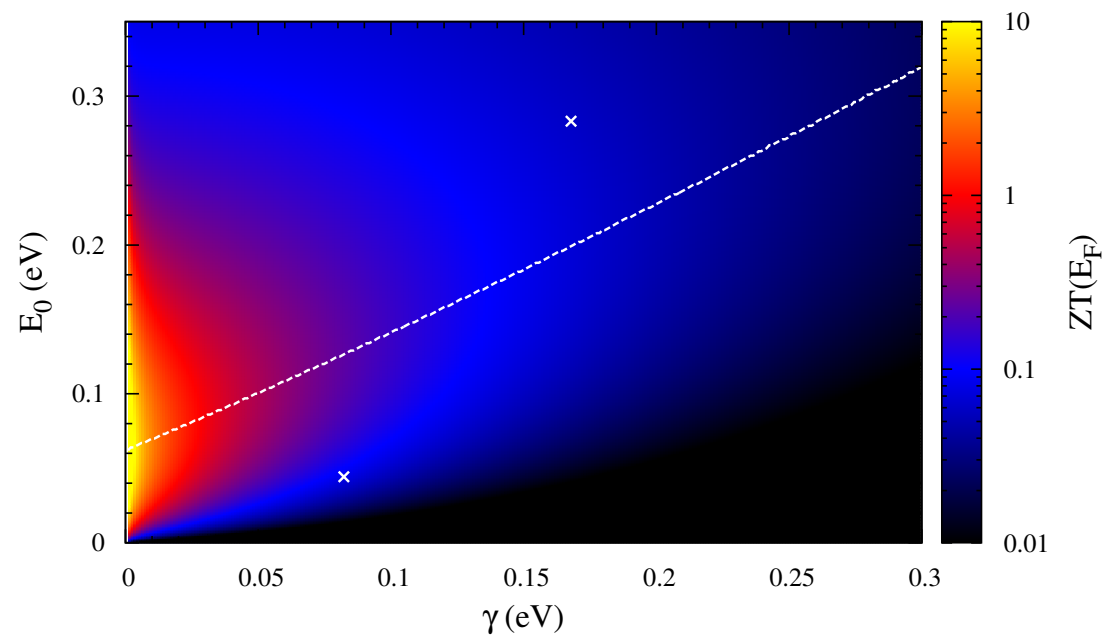

Figura 6.7: Mapa de cor representando a figura de mérito no nível de Fermi, $Z T\left(E_{F}\right)$, para transmitâncias de formato lorentziano em função de sua largura, $\gamma$, e da energia central da lorentziana, $E_{0}$. A linha branca tracejada indica qual $E_{0}$ rende o maior $Z T$ para uma dada largura $\gamma$. Os pontos marcados por $\times$ indicam a largura e posição das lorentzianas obtidas para $L=11.85 \AA$ (superior) e $L=12.35 \AA$ (inferior). Como $\mathcal{L}(E)$ é uma função simétrica em torno de $E_{0}$, plotamos apenas valores para $E_{0} \geqslant 0$. 


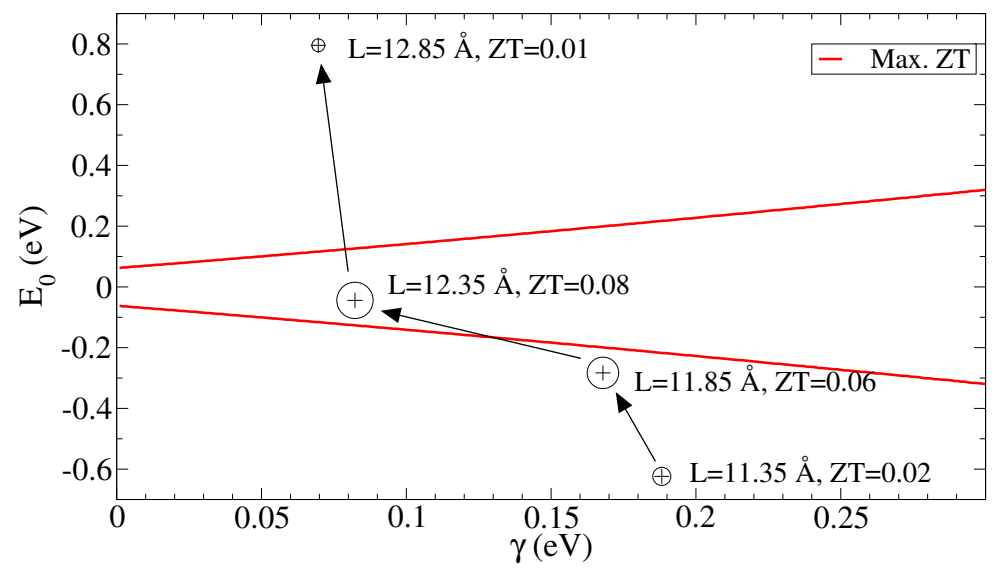

Figura 6.8: Posição, $E_{0}$, e largura, $\gamma$, das funções lorentzianas que ajustam as transmitâncias para vários estiramentos. A área dos círculos em torno dos pontos, marcados por "+", representa a magnitude relativa de $Z T$. Em $L=12.85 \AA$ a junção é rompida.

tos correspondentes a dois comprimentos de separação, $L=11.85 \AA$ e $L=12.35 \AA$ (marcados por "×"). Já na Fig. 6.8 apresentamos os parâmetros, $E_{0}$ e $\gamma$, que ajustam as transmitâncias da junção nas proximidades do ponto de rompimento.

Com o exposto até agora é possível entender como a energia de ressonância do HOMO na transmitância rege as propriedades termoelétricas da junção molecular, em particular, sua figura de mérito. Primeiramente, quanto menor a largura da ressonância em $\mathcal{T}(E)$, maior será sua variação (inclinação) e, portanto, maior será o coeficiente Seebeck (dado o aumento no termo $\mathcal{K}_{1}$ ). Segundo, o pico da transmitância (ou energia da ressonância) deve estar localizado a uma certa distância do nível de Fermi, de forma a maximizar a assimetria local de $\mathcal{T}(E)$ nas proximidades de $E_{F}$. Esta energia $\left(E_{0}\right)$ deve ser tal que a contribuição para o coeficiente Seebeck, $S$, seja máxima de um lado do pico, enquanto a contribuição do lado oposto seja mantida mínima de forma a evitar cancelação devido aos sinais diferentes de $S(E)$ advindos de cada um dos lados do pico na transmitância (lembre que para cada pico em $\mathcal{T}(E)$ equivale uma oscilação em $S(E)$, veja as Figs. 6.2 e 6.3.

Da Fig. 6.6b, podemos ver que, como regra geral, o ponto da transmitância com $\mathcal{T}(E) \cong 0.6 A$ deve estar localizado a aproximadamente $2.5 k_{B} T\left(\cong E_{\delta}\right)$. Solucio- 
nando a Eq.

$$
\frac{A}{1+\left(\frac{E}{\gamma}\right)^{2}}=0.6 A,
$$

vemos que esta regra corresponde a posicionar o pico da transmitância em $E_{0}=$ $E_{\delta}+\sqrt{2 / 3} \gamma$ (veja a Fig. 6.6c).

Então, a receita para maximizar a figura de mérito de uma junção molecular é tentar obter ressonâncias mais estreitas na transmitância, o que pode ser alcançado através de estiramento, como demonstrado anteriormente; e deslocar a energia do nível da molécula (que provoca a ressonância) da forma como descrito acima, o que também é alcançado via estiramento (Fig. 6.2), ou com dopagem (através de grupos doadores ou aceitadores) [115] ou um potencial externo aplicado [125].

Em suma, baseados em cálculos de primeiros princípios, verificamos que o coeficiente Seebeck e a figura de mérito termoelétrica podem ser ajustadas por estiramento mecânico de forma a maximizar a resposta termoelétrica de uma junção Au-BDT$\mathrm{Au}$, que é máxima nas proximidades do ponto de ruptura da junção, quando o HOMO da molécula se aproxima do nível de Fermi. Também apresentamos um esquema simples para otimizar $Z T$ em junções moleculares em geral. 


\section{Capítulo 7}

\section{Conclusões e Perspectivas}

A metodologia para calcular as propriedades termoelétricas de sistemas nanoscópicos no formalismo de Landauer foi implementada com sucesso sobre o código TRANSAMPA. De posse desta ferramenta, estudamos as propriedades termoelétricas de vários sistemas grafíticos, nanofitas de grafeno zigzag pristinas e com impurezas de Boro e Nitrogênio, bicamadas de grafeno e grafeno com dopado com manganês; e a junção molecular Au-BDT-Au.

Entre os principais resultados, vimos que nanofitas pristinas possuem uma figura de mérito baixa $(\sim 0,18)$, implicando em uma baixa eficiência em aplicações termoelétricas (gerador ou refrigerador). No entanto, ao introduzir impurezas substitucionais (Boro ou Nitrogênio) na borda da fita, os estados localizados devido as impurezas causam espalhamento no transporte, resultando em uma queda na transmitância nas regiões de energia associadas aos níveis das impurezas. Estas variações na transmitância fazem com que as propriedades termoelétricas das fitas, dadas pelo coeficiente Seebeck e a figura de mérito $Z \theta$, sejam melhoradas substancialmente, apresentando aumentos de até 2-3 vezes tanto para $S$ quanto para $Z T$.

Outro resultado importante é que, devido à anisotropia de spin na transmitância para as fitas na configuração ferromagnética, as fitas apresentam anisotropia de spin nas suas propriedades termoelétricas. Ou seja, para determinadas energias, 
o coeficiente Seebeck apresenta diferentes valores para elétrons de spin diferentes. Como, no regime linear, a corrente (dependente de spin) é dada por $I_{\sigma}=G_{\sigma} \Delta V_{\sigma}=$ $-G_{\sigma} S_{\sigma} \Delta T$, vemos que é possível gerar uma corrente de spin determinado por efeito Seebeck, no que é chamado de caloritrônica de spin. Também vimos que fitas com impurezas de B ou N podem servir como filtros de spin perfeitos.

Também vimos que o grafeno dopado com manganês, com os Mn presos em monovacâncias, apresenta efeito Seebeck dependente de spin. Propusemos um dispositivo que pode ser ajustado através de um potencial de gate $V_{g}$ de forma a apresentar corrente de um determinado spin (com polarização de spin total) fluindo em uma dada direção, ou correntes para cada spin fluindo em sentidos contrários e sem transporte de carga líquido. É importante notar que neste trabalho é proposto pela primeira vez o uso de grafeno 2D para caloritrônica de spin.

Bicamadas de grafeno, apesar de terem um valor muito baixo de $Z T$ (da ordem de $10^{-3}$ ) quando a contribuição fonônica para a condutividade térmica é incluída, apresentam efeito Seebeck altamente ajustável por efeito de campo, com valores absolutos máximos do coeficiente Seebeck de até $\sim 250 \mu \mathrm{V} / \mathrm{K}$ (valor comparável ao de nanofios de Si [85, 86]).

Para a junção molecular Au-BDT-Au estabelecemos que o coeficiente Seebeck experimenta um aumento dramático quando a junção é esticada, atingindo seu valor máximo na proximidade do ponto de ruptura, quando o HOMO da molécula se aproxima do nível de Fermi. Também propomos um esquema geral para otimizar o $Z T$ de junções moleculares em geral.

\subsection{Perspectivas}

O trabalho realizado nesta tese abriu várias perspectivas muito interessantes e que poderão ser abordadas no futuro. A seguir daremos uma breve descrição de algumas delas (parte já está em andamento). 


\subsubsection{Propriedades termoelétricas de nanofitas com outros defeitos}

No capítulo 3, apresentamos os resultados obtidos para nanofitas com impurezas substitucionais de Boro e Nitrogênio. Outros tipos de defeitos, que são encontrados de forma relativamente comum em sistemas muito semelhantes a nanofitas de grafeno, como nanotubos de Carbono e o próprio grafeno. Estes, são defeitos do tipo Stone-Wales (rotação de ligação), di- e mono-vacâncias [126].

Nas figuras a seguir apresentamos as geometrias (já relaxadas) das reconstruções de alguns defeitos para os quais os cálculos das propriedades de transporte e propriedades termoelétricas já estão em andamento.

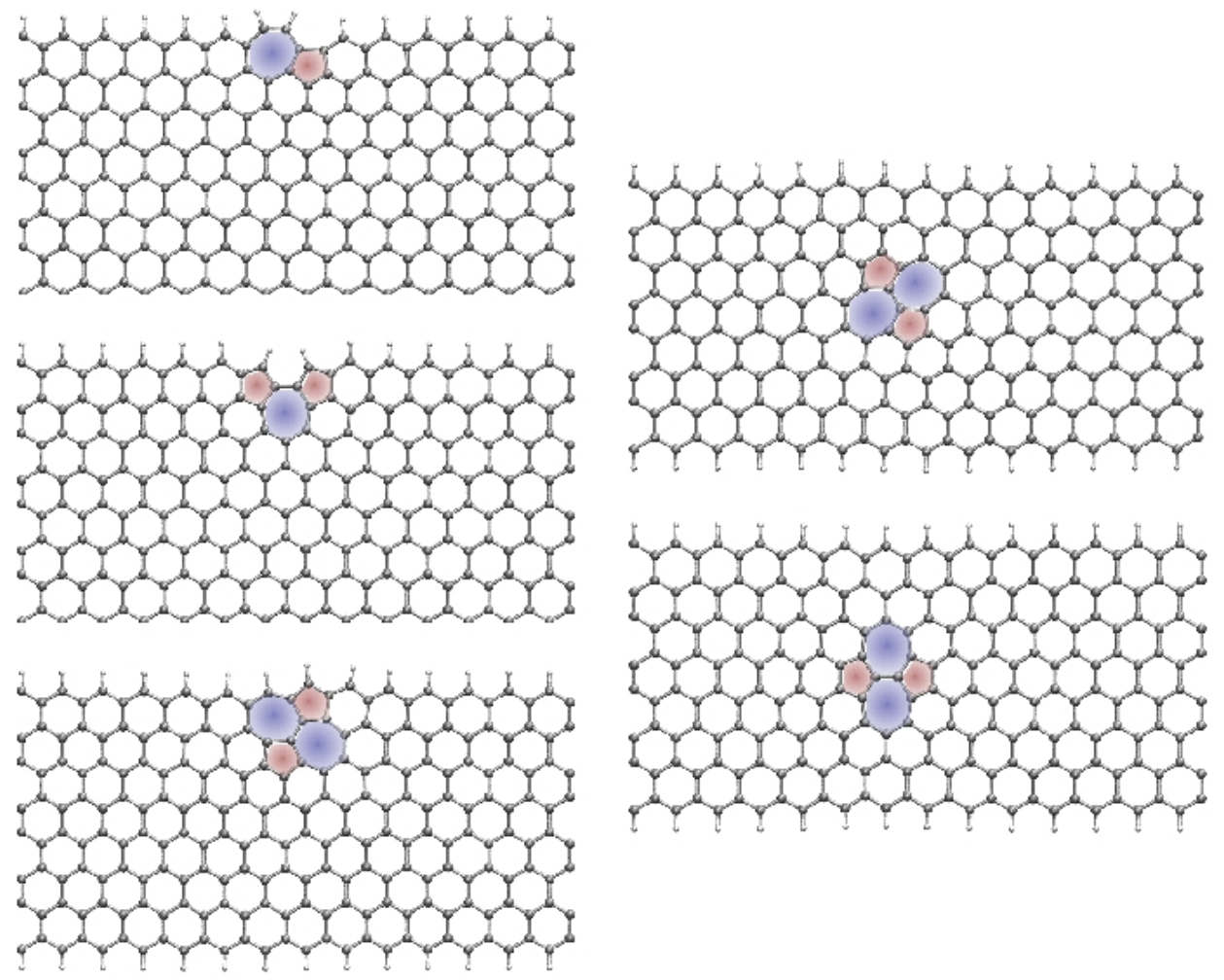

Figura 7.1: Defeitos do tipo Stone-Wales (rotação de ligação) em várias orientações na borda (esquerda) e no centro (direita) de uma nanofita. 

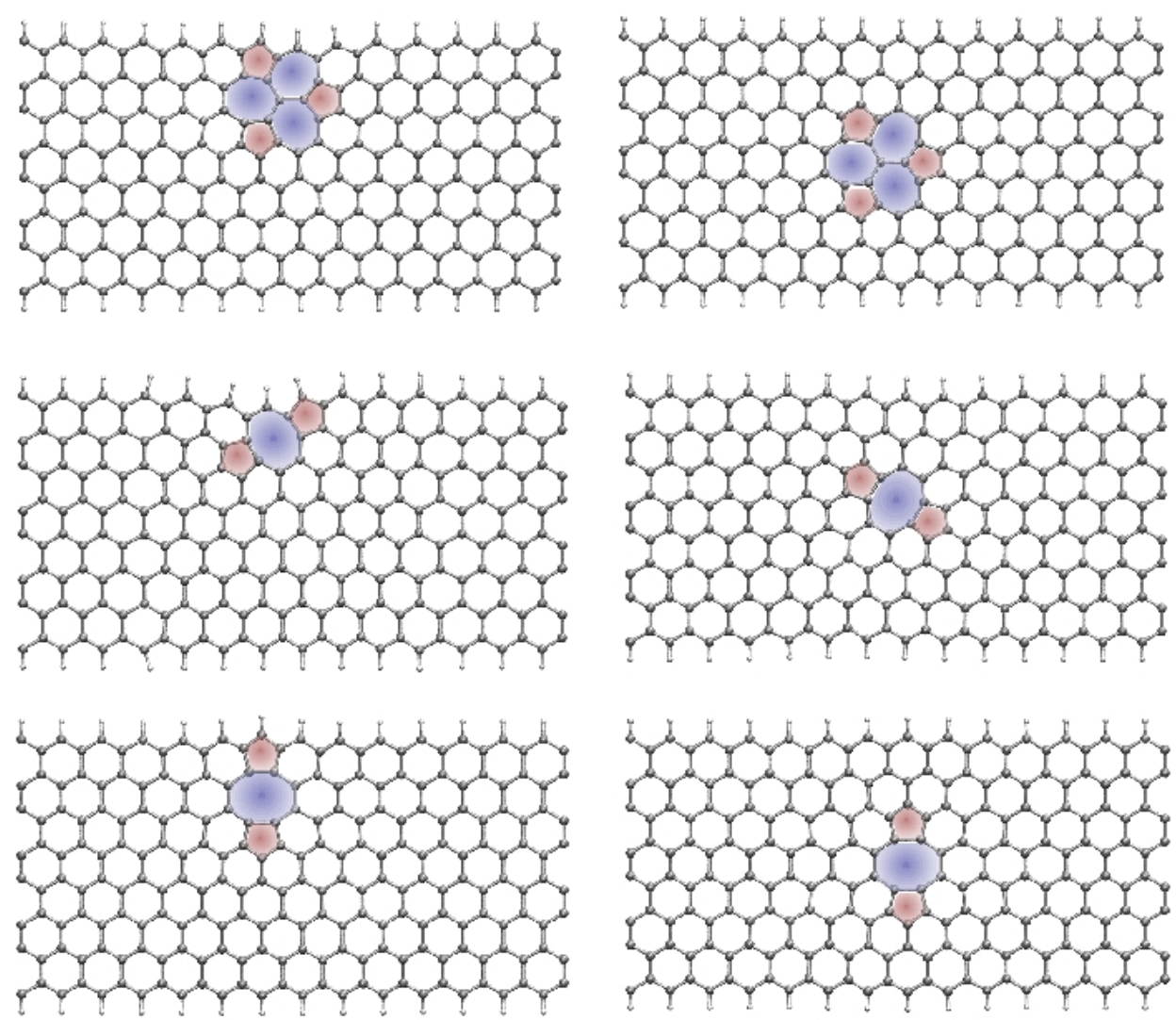

Figura 7.2: Defeitos do tipo divacância nas reconstruções chamadas de 555777 (as duas acima) e 585 em várias orientações na borda (esquerda) e no centro (direita) de uma nanofita.

\subsubsection{Transporte de calor da rede (fônons)}

Como mencionado na seção 2.2, no formalismo de Landauer, a corrente de calor é dada por (Eq. 2.34)

$$
I_{Q, p h}=\frac{1}{h} \int T_{p h}(E) E\left[N\left(E, \Theta_{L}\right)-N\left(E, \Theta_{R}\right)\right] d E
$$

onde transmitância fonônica, $T_{p h}(E)$, pode ser calculada pelo método das funções de Green fora do equilíbrio, da mesma forma que a transmitância eletrônica.

Assim, a transmitância fonônica é dada por 63] 


$$
T_{p h}(\omega)=\operatorname{Tr}\left\{\Gamma_{L} G_{M}^{\dagger} \Gamma_{R} G_{M}\right\}
$$

onde, agora, a função de Green (fonônica) da região central é calculada com a matriz de constantes elástica: $\bigsqcup^{1}$ da região central, $K_{M}$,

$$
G_{M}(\omega)=\left[\left(K_{M}+\Sigma_{L}(\omega)+\Sigma_{R}(\omega)\right)-\omega^{2}\right]^{-1}
$$

As auto-energias, $\Sigma_{L}$ e $\Sigma_{R}$, que representam a influência dos contatos (reservatórios de fônons) na região central, são dadas por

$$
\begin{aligned}
& \Sigma_{L}(\omega)=-K_{M L} g_{L}(\omega) K_{L M} \\
& \Sigma_{R}(\omega)=-K_{M R} g_{R}(\omega) K_{R M},
\end{aligned}
$$

onde a função de Green de superfície do contato esquerdo (direito) é

$$
g_{L(R)}=\left(K_{L(R)}-\omega^{2}\right)^{-1}
$$

Os acoplamentos com os contatos são dados por

$$
\begin{aligned}
\Gamma_{L(R)}(\omega) & =i\left[\Sigma_{L(R)}(\omega)-\Sigma_{L(R)}^{\dagger}(\omega)\right] \\
& =-2 \operatorname{Im}\left\{\Sigma_{L(R)}(\omega)\right\} .
\end{aligned}
$$

Vemos, então, que o procedimento para calcular a a transmitância fonônica, $T_{p h}$, é igual ao usado para calcular a transmitância eletrônica, $T_{e l}$, se substituirmos

\footnotetext{
${ }^{1}$ Aqui já estamos assumindo a aproximação harmônica.
} 
$E \rightarrow \omega^{2}$ e a hamiltoniana eletrônica, $H$, pela matriz de constantes elásticas, $K$,

$$
\left[\begin{array}{ccc}
H_{L} & H_{L M} & 0 \\
H_{M L} & H_{M} & H_{M R} \\
0 & H_{R M} & H_{R}
\end{array}\right] \rightarrow\left[\begin{array}{ccc}
K_{L} & K_{L M} & 0 \\
K_{M L} & K_{M} & K_{M R} \\
0 & K_{R M} & K_{R}
\end{array}\right]
$$

Desta forma, a princípio, todas as ferramentas necessárias para calcular $T_{p h}$ no formalismo de Landauer já se encontram implementadas no código TRANSAMPA. Bastaria apenas fornecer a matriz $K$ para o programa. Esta matriz pode ser calculada com o código SIESTA, o que torna simples, em princípio, a implementação do cálculo da transmitância fonônica.

Uma vez tendo o código implementado, será possível calcular e estudar as propriedades de transporte térmico de sistemas nanoscópicos em conjunto com as propriedades de transporte de carga. Uma das aplicações imediatas para este trabalho será poder calcular a figura de mérito, $Z T$, sem desprezar a condutância térmica da rede, $\kappa_{p h}$.

\subsubsection{Desordem}

Em muitos casos, considerar a presença de desordem se torna necessária se quisermos estudar sistemas mais realistas [127]. Um exemplo seria estudar como a distribuição aleatória de defeitos, como as impurezas de B e N, ao longo de uma nanofita alteraria suas propriedades termoelétricas.

Neste contexto, a desordem pode ser inserida considerando que a região central também é constituída de vários blocos, com interações apenas entre blocos adjacentes, da mesma forma que os contatos (veja a fig. 7.3). Assim, o hamiltoniano da 


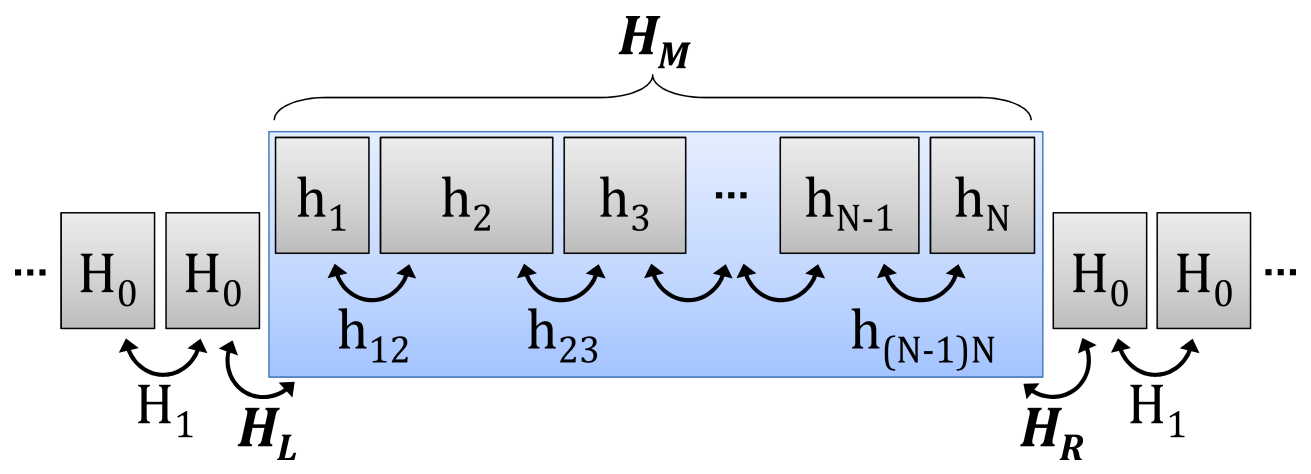

Figura 7.3: Ilustração esquemática da abordagem do problema de desordem.

região central, $H_{M}$, toma a forma

$$
H_{M}=\left[\begin{array}{ccccc}
h_{1} & h_{12} & & & \\
h_{21} & h_{2} & h_{23} & & \\
& h_{32} & h_{3} & \ddots & \\
& & \ddots & \ddots & h_{(N-1) N} \\
& & & h_{N(N-1)} & h_{N}
\end{array}\right]
$$

onde $N$ é o número de blocos que compõem a região central, $h_{i}$, com $i=1, \ldots, N$, são os hamiltonianos de cada bloco e $h_{i j}$ é o acoplamento entre os blocos $i$ e $j$. Eventualmente, muitos desses blocos serão iguais, isto é, representarão uma região pristina ou um mesmo tipo de defeito.

Uma suposição que facilita muito os cálculos é considerar que os acoplamentos entre os blocos $h_{i}$ são iguais ao acoplamento de blocos pristino\$2 Isto evita que seja preciso calcular os acoplamentos entre todos os blocos $h_{i j}$, o que seria uma tarefa muito custosa do ponto de vista computacional.

Na seção 2.1, vimos que a transmitância é dada por (Eq. 2.29

$$
T(E)=\operatorname{Tr}\left\{\Gamma_{L} G^{\dagger} \Gamma_{R} G\right\} .
$$

\footnotetext{
${ }^{2}$ Esta consideração também foi feita para o acoplamento entre os eletrodos e a região central na seção 2.1
} 
A matriz $G$ é densa (e portanto $G^{\dagger}$ ). No entanto, $\Gamma_{L}$ tem a forma

$$
\Gamma_{L}=\left[\begin{array}{ccccc}
\Gamma_{L} & 0 & 0 & \cdots & 0 \\
0 & 0 & 0 & & \vdots \\
0 & 0 & & \ddots & 0 \\
\vdots & & \ddots & \ddots & 0 \\
0 & \cdots & 0 & 0 & 0
\end{array}\right]
$$

e $\Gamma_{R}$,

$$
\Gamma_{R}=\left[\begin{array}{ccccc}
0 & 0 & 0 & \cdots & 0 \\
0 & 0 & 0 & & \vdots \\
0 & 0 & \ddots & \ddots & 0 \\
\vdots & & \ddots & 0 & 0 \\
0 & \cdots & 0 & 0 & \Gamma_{R}
\end{array}\right]
$$

Assim, ao expandir a multiplicação das quatro matrizes e tomar o traço, vemos que apenas precisamos dos elementos da diagonal do sub-bloco $\Gamma_{L} G_{11}^{\dagger} \Gamma_{R} G_{N 1}$. Ou seja, só é preciso conhecer os blocos $G_{11}$ e $G_{N 1}$ de $G_{M}$. Isto nos permite usar a técnica da decimação (descrita no apêndice D), que possibilita calcular apenas esses blocos, sem que seja necessário inverter a matriz $H_{M}$ inteira para obter $G_{M}$ (definida por $\left.G_{M}=(H-E S)^{-1}\right)$.

Com esta abordagem [127, se torna factível calcular as propriedades de transporte de sistemas desordenados e, por conseguinte, suas propriedades termoelétricas. Esta metodologia está sendo implementada sobre o código TRANSAMPA.

\subsubsection{Nanofios/Cadeias Au-Co}

Tendo em vista tudo o que foi aprendido sobre caloritrônica de spin nos trabalhos realizados sobre nanofitas e grafeno com Mn, e a possibilidade de ajustar as propriedades termoelétricas de junções moleculares através de forças, com a reali- 
zação de nanodispositivos eletromecânicos, iniciamos, em colaboração com o Prof. Dr. Renato Borges Pontes (UFGO), um estudo sobre as propriedades termoelétricas de nanofios de $\mathrm{Au}$, próximos do ponto de ruptura, quando formam uma cadeia atômica. Em particular, analisamos como átomos de Co, influenciam o coeficiente Seebeck para cada canal de spin.

Na Figura 7.4 apresentamos resultados parciais já obtidos, as geometrias relaxadas de algumas configurações representativas do estiramento da junção (na parte superior) e a transmitância e coeficiente Seebeck no nível de Fermi em função do alongamento. Pode-se ver que o sistema apresenta efeito Seebeck dependente de spin e que, através de medidas do coeficiente Seebeck é possível diferenciar claramente em qual ligação se dá a ruptura da cadeia. 


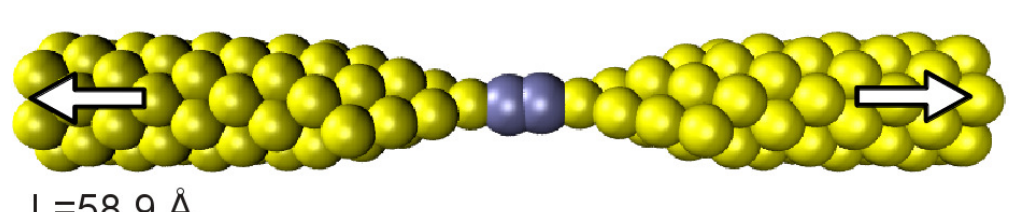

$L=58.9 \AA$

Breaking bond:

-Au-Co- -Co-Au-

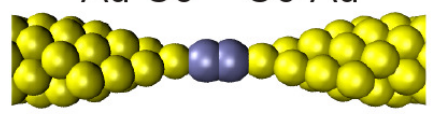

$L=61 \AA$

\section{3 $L=63 \AA$}

Breaking bond: -Au- -Co-Co-Au-
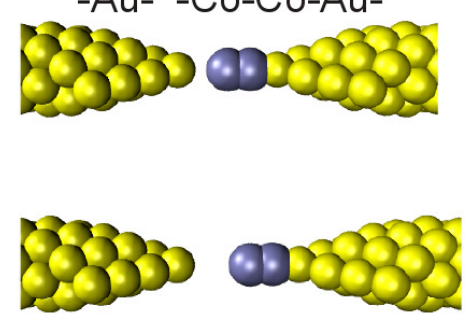

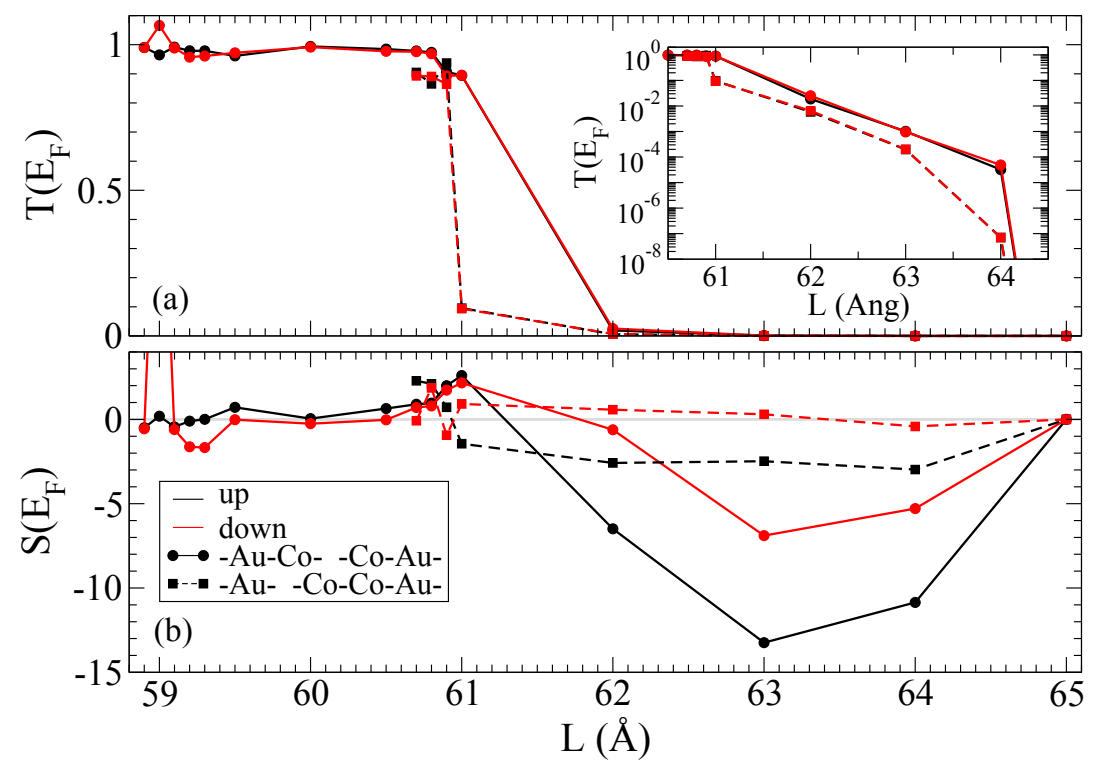

Figura 7.4: Na parte superior, geometrias de algumas configurações representativas do processo de alongamento do nanofio de $\mathrm{Au}$ formando uma cadeia com dois átomos de Co. Na parte inferior, transmitância (a) e coeficiente Seebeck (b) no nível de Fermi, $T\left(E_{F}\right)$ e $S\left(E_{F}\right)$ respectivamente, em função do alongamento da junção. $S\left(E_{F}\right)$ em unidades de $\mu \mathrm{V} / \mathrm{K}$. 


\section{Apêndice A}

\section{Teoria do Funcional da Densidade}

Nosso conhecimento da estrutura eletrônica da matéria é baseado em modelos quânticos e estatísticos. Para obter estas propriedades deve-se resolver a equação de Schrödinger. Assim, desde o surgimento da mecânica quântica, resolver a equação de Schrödinger para um sistema de muitos corpos interagentes tem constituído um problema fundamental, e ao mesmo tempo, de difícil solução.

O hamiltoniano para um sistema composto de elétrons e núcleos é dada por

$$
H=T_{e}+V_{e N}+V_{e e}+T_{N}+V_{N N}
$$

onde o termo

$$
T_{e}=-\frac{\hbar}{2 m_{e}} \sum_{i} \nabla_{i}^{2}
$$

é a energia cinética dos elétrons,

$$
V_{e N}=-\sum_{i, I} \frac{Z_{I} e^{2}}{\left|\mathbf{r}_{i}-\mathbf{R}_{I}\right|}
$$

representa a interação entre os elétrons e os núcleos,

$$
V_{e e}=-\frac{1}{2} \sum_{i \neq j} \frac{e^{2}}{\left|\mathbf{r}_{i}-\mathbf{r}_{j}\right|},
$$


a interação inter-eletrônica,

$$
T_{N}=-\sum_{I} \frac{\hbar}{2 M_{I}} \nabla_{I}^{2}
$$

é a energia cinética devida aos núcleos e, por último,

$$
V_{N N}=-\frac{1}{2} \sum_{I \neq J} \frac{Z_{I} Z_{J} e^{2}}{\left|\mathbf{R}_{i}-\mathbf{R}_{j}\right|},
$$

é a interação entre núcleos.

Usando a aproximação de Born-Oppenheimer (apêndice B), podemos considerar os núcleos como sendo fixos e re-escrever o hamiltoniano como

$$
H_{B O}=T_{e}+V_{e e}+V_{e x t},
$$

onde, agora, as posições dos núcleos são consideradas parâmetros. O termo

$$
V_{e x t}=-\sum_{i, I} V_{I}\left(\left|\mathbf{r}_{i}-\mathbf{R}_{I}\right|\right)=V_{e N}
$$

está associado com o potencial externo, indentificado como $V_{e N}$ na Eq. A.3.

Assim, para obter a estrutura eletrônica deste sistema, é necessário resolver a equação de Schrödinger (independente do tempo) com o hamiltoniand 1 (A.7),

$$
H|\Psi\rangle=\left(T_{e}+V_{e e}+V_{e x t}\right)|\Psi\rangle=E|\Psi\rangle .
$$

É interessante notar que os termos $T_{e}$ e $V_{e e}$ são sempre iguais para qualquer sistema, isto é, são universais. Desta forma, o problema é especificado pelo potencial externo $V_{\text {ext }}$.

A grande dificuldade em resolver esta equação reside no termo $V_{e e}$ (Eq. A.4,

\footnotetext{
${ }^{1}$ Daqui em diante o subescrito BO será abandonado.
} 
que acopla o conjunto de variáveis $\left\{\mathbf{r}_{i}\right\}$, e faz com que seja extremamente difícil encontrar soluções analíticas. Diversos métodos de primeiros princípios, i.e. sem parâmetros ajustáveis, foram idealizados para conseguir resolver a Eq. A.8), como a aproximação de Hartree-Fock, Interação de Configurações e Coupled Cluster [128].

Estes métodos empregam aproximações para a função de onda a fim de resolver o problema de muitos corpos. Uma vez obtidas as funções de onda, todos os observáveis podem ser calculados. No entanto, como a função de onda é função de $3 \mathrm{~N}$ variáveis, o custo computacional para tratar sistemas grandes é muito alto, tornando-se inviável.

A teoria do funcional da densidade (Density Functional Theory - DFT), por outro lado, parte de uma abordagem completamente diferente. Na DFT, a variável básica não é mais a função de onda, mas a densidade eletrônica, uma função de três coordenadas. A partir dela, todos os observáveis podem ser obtidos, em princípio.

As primeiras tentativas para formular uma teoria do funcional da densidade para sistema quânticos começaram em 1927, com os trabalhos de Thomas [129] e Fermi [130], que propuseram uma aproximação para calcular a energia cinética dos elétrons em função da densidade, enquanto que a interação inter-eletrônica era tratada classicamente. O funcional de energia de Thomas-Fermi é dado por

$$
E_{T F}[n]=T_{T F}[n]+U_{\text {Hartree }}[n]+V_{\text {ext }}[n],
$$

onde

$$
U_{\text {Hartree }}[n]=\frac{1}{2} \int d \mathbf{r} d \mathbf{r}^{\prime} \frac{n(\mathbf{r}) n\left(\mathbf{r}^{\prime}\right)}{\left|\mathbf{r}-\mathbf{r}^{\prime}\right|}
$$

é a energia de Hartree, o termo clássico da interação entre elétrons, e é usada uma aproximação de densidade local (Local Density Approximation - LDA) para a energia cinética,

$$
T_{T F}[n]=\int d \mathbf{r} t_{s}^{h o m}(\mathbf{r}) n(\mathbf{r})=\frac{3}{10}\left(3 \pi^{2}\right)^{(2 / 3)} \int d \mathbf{r} n(\mathbf{r})^{(5 / 3)}
$$


isto é, considera-se que a densidade de energia cinética num dado ponto do espaço do sistema original é igual à de um gás homogêneo de elétrons, $t(\mathbf{r}) \approx t^{\text {hom }}(\mathbf{r})$.

Posteriormente, Dirac estendeu o modelo incluindo um termo de troca usando, também, a aproximação LDA. Também foram propostas correções para levar em conta inomogeneidades na densidade. No entanto, o modelo de Thomas-Fermi emprega aproximações muito cruas, fornecendo resultados não muito bons na maioria dos casos [29]. O maior mérito deste modelo foi o de usar a densidade como variável básica, sugerindo que seria possível sair de um problema de $3 \mathrm{~N}$ coordenadas (as funções de onda) para um de apenas três (a densidade).

\section{A.1 Teoremas de Hohenberg-Kohn}

Os teoremas de Hohenberg-Kohn (HK) [131] estabeleceram a fundamentação teórica da DFT. Em sua versão original, eles foram derivados para sistemas com estado fundamental não-degenerado e sem polarização de spin. Contudo, existem extensões para tratar sistemas com spin e com degenerescências no estado fundamental [29].

O primeiro teorema garante que a densidade eletrônica do estado fundamental, $n_{0}(\mathbf{r})$, determina univocamente (a menos de uma constante) o potencial externo $2^{2}$ $V_{\text {ext }}$. Conseqüentemente, como $V_{\text {ext }}$ determina o hamiltoniano, e este, todas as funções de onda, temos que todas as propriedades do sistema são completamente determinadas pela densidade do estado fundamental $n_{0}(\mathbf{r})$.

O segundo teorema nos diz que um funcional universal da energia em função da densidade, $E[n]$, pode ser definido. E que este, para um dado potencial externo $V_{\text {ext }}$, possui um mínimo global para a densidade do estado fundamental $n_{0}(\mathbf{r})$, ou seja, $E\left[n_{0}\right]<E\left[n^{\prime}\right]$ para qualquer densidade $n^{\prime} \neq n_{0}$. Uma conseqüência direta deste teorema é que o funcional de densidade $E[n]$ é suficiente para determinar a

\footnotetext{
${ }^{2} \mathrm{O}$ teorema é válido apenas para densidades $n_{0}$ que correspondem a algum potencial externo $V_{\text {ext }}$. Densidades deste tipo são ditas $v$-representáveis.
} 
Schrödinger

Difícil

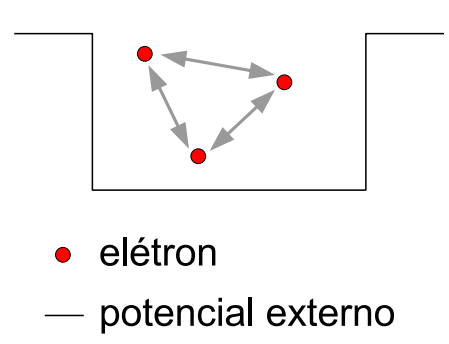

DFT Kohn-Sham

"Fácil"

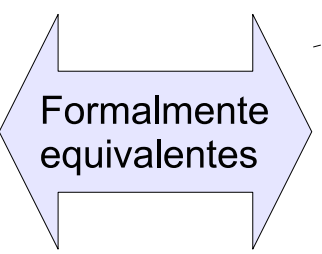

- partícula não-interagente

-.. potencial efetivo

Figura A.1: Ilustração pictórica do método de Kohn-Sham. O sistema de elétrons interagentes (esquerda) é mapeado em um sistema de partículas não interagentes em um potencial efetivo (direita).

densidade do estado fundamental $n_{0}$ e sua energia, $E_{0} \equiv E\left[n_{0}\right]$.

Assim, sabemos que existe um funcional da densidade,

$$
E_{H K}[n]=T[n]+V_{e e}[n]+V_{e x t}[n],
$$

com o qual podemos obter $n_{0}$ e $E_{0}$ por métodos variacionais. No entanto, os teoremas de HK apenas nos informam da existência deste funcional, não fornecendo nenhuma pista sobre como construí-lo na prática.

\section{A.2 Ansatz de Kohn-Sham}

Hoje em dia, a DFT é o método mais usado e um dos mais bem sucedidos para cálculos de estrutura eletrônica. Isto se deve graças à proposta feita por Kohn e Sham [132] em 1965, sem a qual DFT, apesar de ser muito interessante do ponto de vista teórico, não teria muita utilidade na prática. A idéia principal de seu esquema consiste em mapear o problema de muitos corpos interagentes em um problema não-interagente.

A dificuldade em se calcular o funcional de energia de HK é que não se conhecem expressões gerais para $T_{e}[n]$ e $V_{e e}[n]$. No entanto, sabemos calcular a energia cinética 
através das funções de onda (da forma usual),

$$
T=\left\langle\Psi\left|-\frac{\hbar}{2 m} \sum_{i} \nabla_{i}^{2}\right| \Psi\right\rangle
$$

e a parte clássica da interação inter-eletrônica (Eq. A.10).

Considere, então, um sistema auxiliar de N partículas não-interagentes $3^{3}$ sujeitos a um potencial externo $V_{s}$. A hamiltoniana deste sistema é

$$
H_{s}=T_{s}+V_{s}=-\frac{\hbar}{2 m} \sum_{i} \nabla_{i}^{2}+V_{s}
$$

e sua solução é dada pelo conjunto de equações

$$
\left(-\frac{\hbar}{2 m} \nabla^{2}+V_{s}(\mathbf{r})\right) \varphi_{i}(\mathbf{r})=\epsilon_{i} \varphi_{i}(\mathbf{r})
$$

Do teorema de HK, sabemos que existe um funcional de energia único para este sistema,

$$
E_{s}[n]=T_{s}[n]+V_{s}[n]
$$

Comparando com a Eq. A.12 vemos que se substituirmos $V_{s}$ por um potencial efetivo $V_{\text {eff }}=V_{\text {Hartree }}+V_{\text {ext }}$ na Eq. acima, teremos

$$
\begin{aligned}
E_{s}[n] & =T_{s}[n]+V_{\text {eff }}[n] \\
& =T_{s}[n]+U_{\text {Hartree }}[n]+V_{\text {ext }}[n] .
\end{aligned}
$$

A diferença entre os funcionais de energia dos sistemas interagente e não-interagente (com o potencial efetivo $V_{e f f}$ ), que é devida à efeitos de troca e correlação, é chamada

\footnotetext{
${ }^{3}$ Usaremos o subescrito s (do inglês single) para denotar o sistema não-interagente.
} 
de funcional de troca e correlação, $E_{x c}[n]$, definido como

$$
\begin{aligned}
E_{x c}[n] & =E[n]-E_{s}[n] \\
& =T[n]-T_{s}[n]+V_{e e}[n]-V_{\text {Hartree }}[n] .
\end{aligned}
$$

Então, é fácil ver que se adicionarmos o funcional de troca e correlação ao potencial efetivo, teremos $E_{s}[n]=E[n]$.

Definindo, finalmente, o potencial efetivo do sistema auxiliar KS como

$$
V_{e f f}^{K S}=U_{\text {Hartree }}+V_{e x t}+E_{x c}
$$

obtemos um funcional de energia igual ao do sistema interagente,

$$
E_{K S}[n]=T_{s}[n]+V_{\text {Hartree }}[n]+V_{\text {ext }}[n]+E_{x c}[n]=E[n] .
$$

Assim, o ansatz de Kohn-Sham consiste em assumir que a densidade do estado fundamental do sistema de muitos corpos interagentes, além de ser $v$-representável, também é não-interagente- $v$-representável, ou seja, que a densidade do estado fundamental, além de ser representada pelo potencial $V_{e x t}$, também é representada por um potencial não-interagente, isto é, que $n_{0}(r)=n_{s}(r)$. Para que seja possível calcular a energia cinética $T_{s}$, utilizam-se as funções de onda, dadas pelas soluções de

$$
\left(-\frac{\hbar}{2 m} \nabla^{2}+V_{e f f}^{K S}(\mathbf{r})\right) \varphi_{i}^{K S}(\mathbf{r})=\epsilon_{i}^{K S} \varphi_{i}^{K S}(\mathbf{r})
$$

Nestas equações, o potencial efetivo depende da densidade, e portanto, elas são resolvidas de forma auto-consistente. É importante ressaltar que as autofunções $\varphi_{i}^{K S}$ e os autovalores $\epsilon_{i}^{K S}$ (chamados de orbitais e energias Kohn-Sham) que aparecem em A.21 são as funções de onda e energias do sistema auxiliar não-interagente, introduzido como um artifício matemático para possibilitar o cálculo da densidade do 
sistema interagente. Assim, estes são descarecidos de significado físicd $\mathbb{4}^{4}$ fornecendo apenas a densidade e energia do estado fundamental corretas. Apesar disso, a experiência tem mostrado que, muitas vezes, os orbitais e energias Kohn-Sham fornecem boas aproximações para as funções de onda e energias das excitações elementares do sistema interagente.

A grande vantagem da abordagem de Kohn-Sham está no fato de isolar em um termo separado, o funcional de troca e correlação, $E_{x c}$, os efeitos de muitos corpos, que são difíceis calcular. Tipicamente, este termo é pequeno em comparação a $T_{s}$, $V_{\text {Hartree }}$ e $V_{\text {ext }}$. Logo, espera-se que mesmo aproximações simples para $E_{x c}$ forneçam bons resultados.

\section{A.3 Aproximação da Densidade Local (LDA)}

A primeira aproximação para o termo de troca e correlação também foi sugerida por Kohn e Sham [132] no mesmo artigo no qual propuseram o método que hoje leva seus nomes. Esta, da mesma forma que no caso da energia cinética no modelo de Thomas-Fermi (Eq. A.11), aproxima localmente a energia de troca e correlação do sistema pela de um gás de elétrons homogêneo com a mesma densidade.

A expressão da energia de troca para um gás de elétrons homogêneo é conhecida analiticament $\oiiint^{5}$

$$
\begin{aligned}
E_{x}^{L D A}[n] & =\int d \mathbf{r} \varepsilon_{x}^{h o m}(n(\mathbf{r})) n(\mathbf{r}) \\
& =-\frac{3 e^{2}}{4}\left(\frac{3}{\pi}\right)^{\frac{1}{3}} \int d \mathbf{r} n(\mathbf{r})^{4 / 3}
\end{aligned}
$$

O mesmo não acontece para a energia de correlação. No entanto, Ceperley e Al-

\footnotetext{
[29.

${ }^{4}$ Com exceção do último autovalor ocupado, que corresponde ao negativo da energia de ionização

${ }^{5}$ Adicionando o termo $E_{x}^{L D A}[n]$ ao modelo de Thomas-Fermi obtém-se o funcional de ThomasFermi-Dirac.
} 
der (CA) realizaram cálculos precisos [133], baseados em métodos de Monte Carlo quântico, da energia de correlação do gás uniforme de elétrons. Cálculos modernos usam parametrizações dos dados publicados por CA para calcular $\varepsilon_{c}^{\text {hom }}$ [134].

Assim, temos que o funcional de troca e correlação na aproximação LDA é

$$
E_{x c}^{L D A}[n]=\int d \mathbf{r}\left[\varepsilon_{x}^{h o m}(n(\mathbf{r}))+\varepsilon_{c}^{h o m}(n(\mathbf{r}))\right] n(\mathbf{r})
$$

Por ser baseado num gás de elétrons homogêneo, espera-se que a aproximação LDA funcione melhor para sistemas mais homogêneos (como metais), com densidades que variam lentamente, do que para átomos ou moléculas, onde há grandes variações da densidade.

De forma geral, a LDA tende a superestimar energias de ligação em moléculas e de coesão em sólidos e subestimar comprimentos de ligação. Apesar disso, surpreendentemente (devido à sua simplicidade) a LDA fornece bons resultados mesmo em casos onde há grandes variações da densidade.

\section{A.4 Aproximações Generalizadas de Gradiente (GGA)}

O sucesso da LDA impulsionou o desenvolvimento de funcionais de troca e correlação que, além do valor densidade em um ponto do espaço, levassem em conta informações sobre a variação da mesma há grandes variações da densidade. Estas aproximações para $E_{x c}[n]$, chamadas de aproximações generalizadas de gradiente, são dadas, na sua forma genérica, por

$$
E_{x c}^{G G A}[n]=\int d \mathbf{r} \varepsilon_{x c}^{G G A}(n, \nabla n) n(\mathbf{r})
$$

Diferentemente da LDA, cujo funcional é universal (há apenas variações na forma de parametrizar os dados de CA), há uma grande variedade de funcionais GGA. Os

\footnotetext{
${ }^{6} \mathrm{Na}$ prática, nenhum sistema é uniforme como supõe o modelo de gás homogêneo.
} 
mais usados em química quântica costumam ser ajustados por algum conjunto de dados experimentais, sendo chamados de semi-empíricos. Já os mais usados em física costumam ser baseados em vínculos e limites assintóticos exatos. Entre os funcionais mais usados podemos citar os propostos por Perdew, Burke e Erzenhof (PBE) [106], Perdew e Wang (PW91) [135] e o BLYP [136, 137] (que é semi-empírico). Genericamente, embora não de forma sistemática, os funcionais GGA melhoram a descrição das energias atômicas, de ligação e coesão, e comprimentos e ângulos de ligação.

Além dos funcionais LDA e GGA existem os chamados funcionais híbridos, que misturam a troca e correlação de funcionais LDA, GGA e a troca exata de HartreeFock, em geral ajustando dados experimentais. Um exemplo de funcional híbrido muito usado atualmente é o B3LYP [29]. Outra classe de funcionais são os metaGGAs, que incluem informação sobre o laplaciano da densidade, $\nabla^{2} n$, além da própria densidade e seu gradiente. 


\section{Apêndice B}

\section{Aproximação de Born-Oppenheimer}

O hamiltoniano de um sistema composto por elétrons e núcleos (Eq. A.1) pode ser reescrita como

$$
\begin{aligned}
H & =T_{N}+T_{e}+U \\
& =T_{N}+H_{e}
\end{aligned}
$$

onde $T_{N}$ é a energia cinética dos núcleos, dada por

$$
T_{N}=-\sum_{I} \frac{\hbar^{2}}{2 M_{I}} \nabla_{I}^{2},
$$

$H_{e}$ é a hamiltoniana eletrônica, composta dos termos $T_{e}$, a energia cinética eletrônica,

$$
T_{e}=-\frac{\hbar^{2}}{2 m_{e}} \sum_{i} \nabla_{i}^{2}
$$

e $U=V_{e N}+V_{e e}+V_{N N}$, que agrupa as energias potenciais,

$$
V_{e N}=-\sum_{I, i} \frac{Z_{I} e^{2}}{\left|\mathbf{R}_{I}-\mathbf{r}_{i}\right|}
$$




$$
\begin{aligned}
V_{e e} & =-\frac{1}{2} \sum_{i \neq j} \frac{e^{2}}{\left|\mathbf{r}_{i}-\mathbf{r}_{j}\right|} \\
V_{N N} & =\frac{1}{2} \sum_{I \neq J} \frac{Z_{I} Z_{J} e^{2}}{\left|\mathbf{R}_{I}-\mathbf{R}_{J}\right|} .
\end{aligned}
$$

A solução para este sistema, que acopla as variáveis eletrônicas e nucleares através de $V_{e N}$, é dada por

$$
H \Psi_{s}(\mathbf{r}, \mathbf{R})=E_{s} \Psi_{s}(\mathbf{r}, \mathbf{R})
$$

Vamos supor que as soluções da parte eletrônica são conhecidas,

$$
H_{e} \psi_{i}(\mathbf{r} ; \mathbf{R})=E_{i}(\mathbf{R}) \psi_{i}(\mathbf{r} ; \mathbf{R})
$$

onde $\psi_{i}$ e $E_{i}$ dependem parametricamente das coordenadas nucleares R. Então, para cada $\mathbf{R}$ as autofunções $\psi_{i}$ formam uma base completa ( $H_{e}$ é hermitiano) para os estados eletrônicos. Logo, podemos escrever a solução para o sistema acoplado como uma combinação linear das funções de onda eletrônicas,

$$
\Psi_{s}(\mathbf{r}, \mathbf{R})=\sum_{i} \chi_{s i}(\mathbf{R}) \psi_{i}(\mathbf{r} ; \mathbf{R})
$$

Inserindo esta expressão em (B.7), multiplicando $\psi_{j}^{*}(\mathbf{r} ; \mathbf{R})$ e integrando nas variáveis eletrônicas, obtemos

$$
\left[T_{N}+E_{i}(\mathbf{R})-E_{s}\right] \chi_{s i}(\mathbf{R})=-\sum_{j} C_{i j}(\mathbf{R}) \chi_{s j}(\mathbf{R})
$$

onde $C_{i j}=A_{i j}+B_{i j}$ com

$$
\begin{aligned}
A_{i j}(\mathbf{R}) & =2\left(\sqrt{T_{N}}\right)_{i j} \sqrt{T_{n}} \\
& =\sum_{I} \frac{1}{M_{I}} \int \psi_{i}^{*}(\mathbf{r} ; \mathbf{R}) \nabla_{I} \psi_{j}(\mathbf{r} ; \mathbf{R}) d \mathbf{r} \nabla_{I}
\end{aligned}
$$


$\mathrm{e}$

$$
\begin{aligned}
B_{i j}(\mathbf{R}) & =2\left(T_{N}\right)_{i j} \\
& =\sum_{I} \frac{1}{2 M_{I}} \int \psi_{i}^{*}(\mathbf{r} ; \mathbf{R}) \nabla_{I}^{2} \psi_{j}(\mathbf{r} ; \mathbf{R}) d \mathbf{r} .
\end{aligned}
$$

Na aproximação de Born-Oppenheimer todos os termos $C_{i j}$ são considerados nulos. Já a chamada aproximação adiabática consiste em desprezar os termos não diagonais $C_{i j}, i \neq j$, que acoplam diferentes estados eletrônicos, mantendo os termos diagonais. Ou seja, é assumido que os elétrons permanecem num mesmo estado enquanto os núcleos se movem. Esta aproximação é geralmente justificada pela grande diferença de massa entre os núcleos e os elétrons $\left(m_{e} / M \ll 1\right)$.

Assim, na aproximação adiabática, o movimento nuclear é descrito por

$$
\left[-\sum_{I} \frac{1}{2 M_{I}} \nabla_{I}^{2}+U_{i}(\mathbf{R})\right] \chi_{n i}(\mathbf{R})=E_{n i} \chi_{n i}(\mathbf{R}),
$$

onde $U_{i}(\mathbf{R})=E_{i}(\mathbf{R})+B_{i i}(\mathbf{R})$ determina um potencial para os núcleos ${ }^{1}$ e o índice $n$ se refere a estados nucleares.

\footnotetext{
${ }^{1} \mathrm{O}$ requerimento de que $\psi_{i}$ seja normalizado implica $A_{i i}=0$.
} 


\section{Apêndice C}

\section{Pseudopotenciais}

Em geral, em um átomo, apenas os elétrons da camada de valência são quimicamente ativos, participando da determinação das propriedades de sólidos e moléculas. Já os elétrons de caroço, que estão fortemente ligados ao núcleo, são quimicamente inertes, dependendo pouco do ambiente químico no qual o átomo se encontra.

Valendo-se disso, a aproximação de pseudopotencial consiste substituir o potencial gerado pelos elétrons de caroço mais o núcleo por um potencial efetivo dentro da região de caroço, definida por um raio de corte $r_{c}$ (veja a fig. C.1). O pseudopotencial, $V_{P S}$, é mais fraco que o original na região $r<r_{c}$ porque os elétrons de caroço blindam o potencial nuclear. Fora da região de caroço $\left(r>r_{c}\right), V_{P S}=V$. Desta forma, o número de elétrons é reduzido nos cálculos, diminuindo o custo computacional.

Um aspecto importante referente aos pseudopotencias, ao qual deve ser dada atenção, é sua tranferibilidade. Esta é definida como a capacidade de descrever adequadamente ambientes químicos diferentes daqueles nos quais o pseudopotencial foi criadd ${ }^{1}$. Como regra, quanto maior o raio de corte de um pseudopotencial, mais suave este será, permitindo um ganho computacional maior. Por outro lado, ele se torna menos transferível. Assim, há uma competição entre performance e

\footnotetext{
${ }^{1}$ Em geral, pseudopotenciais são gerados em cálculos atômicos.
} 


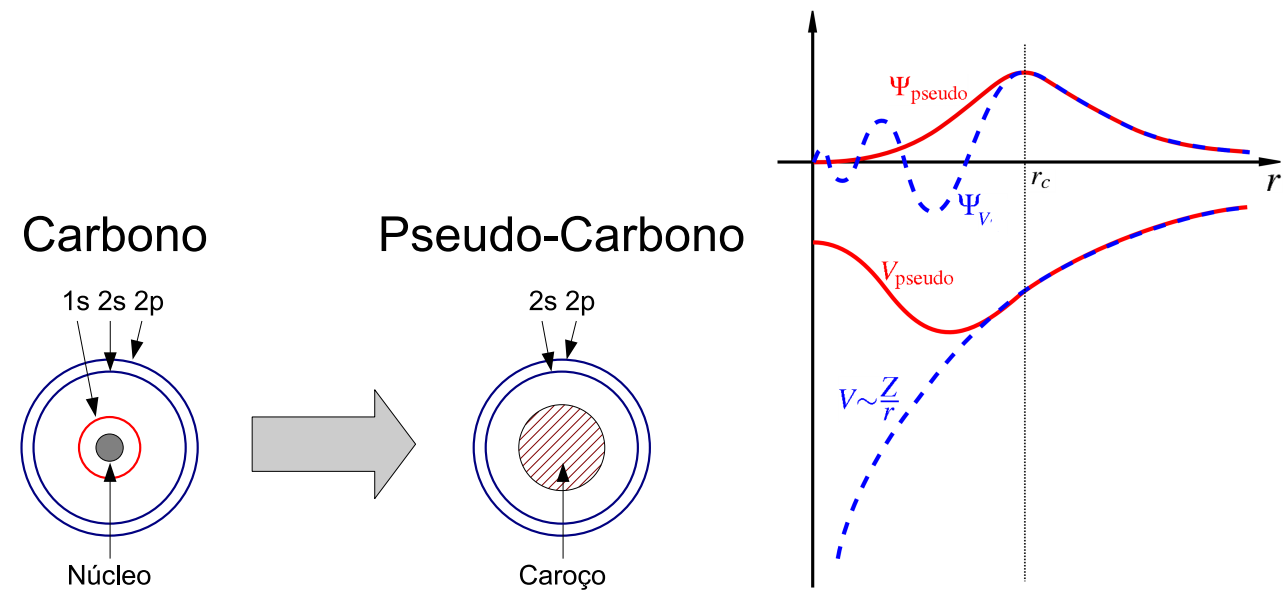

Figura C.1: Filosofia da aproximação de pseudopotencial. - Representação esquemática do pseudopotencial $V_{\text {pseudo }}$ e pseudo-função de onda $\Psi_{\text {pseudo }}$ (em vermelho), e do potencial $V(Z / r)$ e função de onda correspondente, $\Psi_{V}$ (azul). A partir do raio de corte $r_{c}$, as funções $\Psi_{\text {pseudo }}$ e $\Psi_{V}$ coincidem.

transferibilidade, dada pelo raio de corte $r_{c}$. 


\section{Apêndice D}

\section{Decimação}

A decimação é uma técnica matemática muito simples e que permite conhecer uma parte da inversa de uma matriz sem precisar calcular a matriz inversa por inteiro.

Considere as matrizes $A$ e $B$ tal que $B=A^{-1}$. Assim, temos que

$$
\left[\begin{array}{ll}
A_{11} & A_{12} \\
A_{21} & A_{22}
\end{array}\right]\left[\begin{array}{ll}
B_{11} & B_{12} \\
B_{21} & B_{22}
\end{array}\right]=\left[\begin{array}{ll}
1 & 0 \\
0 & 1
\end{array}\right]
$$

onde os elementos $A_{i j}$ e $B_{i j}$ podem, de forma geral, ser submatrizes. Uma forma de determinar $B$ é resolver o sistema de equações definido por (D.1), que é dado por

$$
\left\{\begin{array}{l}
A_{11} B_{11}+A_{12} B_{21}=1 \\
A_{11} B_{12}+A_{12} B_{22}=0 \\
A_{21} B_{11}+A_{22} B_{21}=0 \\
A_{21} B_{12}+A_{22} B_{22}=1
\end{array} .\right.
$$

Suponha que desejamos conhecer, por exemplo, apenas o elemento $B_{11}$, ou, equivalentemente, que não queremos conhecer os outros elementos. Este elemento aparece apenas na primeira e terceira equações do sistema (D.2). Consequentemente, preci- 
samos resolver apenas metade do sistema de equações:

$$
\left\{\begin{array}{l}
A_{11} B_{11}+A_{12} B_{21}=1 \\
A_{21} B_{11}+A_{22} B_{21}=0
\end{array} .\right.
$$

Resolvendo a segunda equação do sistema (D.3) para $B_{21}$, obtemos

$$
B_{21}=-A_{22}^{-1} A_{21} B_{11}
$$

Desta forma, reescrevemos o elemento $B_{21}$ em função de $B_{11}$. Inserindo este resultado na primeira Eq. do sistema (D.3), temos

$$
B_{11}=\left(A_{11}-A_{12} A_{22}^{-1} A_{21}\right)^{-1}
$$

Assim, vemos que para determinar $B_{11}$ não é necessário inverter a matriz $A$ por completo. No entanto, é preciso inverter o bloco $A_{22}$, multiplicá-lo por $A_{21}$ pela direita e por $A_{12}$ pela esquerda, subtrair este resultado de $A_{11}$ e, finalmente, fazer outra inversão.

Naturalmente, surge a pergunta: Será que está técnica realmente compensa? A princípio, pelo visto no desenvolvimento acima, o sistema de equações a ser resolvido (Eq. D.3) possui metade das Eqs. do sistema original (Eq. D.2). No entanto, a partir da Eq. D.5 não fica claro se há um ganho real. Considerando que o número de operações para realizar uma multiplicação de matrizes é $N^{3}$, e para realizar uma inversão é $C_{I n v} N^{3}$, onde $C_{I n v} \approx 1.3$ é uma constante definida pelo algoritmo usado na inversão (veja a fig. D.1); para que a decimação seja mais rápidd ${ }^{1}$ que a inversão, devemos ter

$$
C_{\text {Inv }} N_{2}^{3}+2 N_{2}^{2} N_{1}+N_{1}^{2}+C_{\text {Inv }} N_{1}^{3} \leqslant C_{\text {Inv }} N^{3}
$$

onde $N_{1}$ e $N_{2}$ são as dimensões dos blocos $A_{11}$ e $A_{22}$ respectivamente. Como obvia-

\footnotetext{
${ }^{1}$ Considerando que o tempo de cálculo seja proporcional ao número de operações.
} 


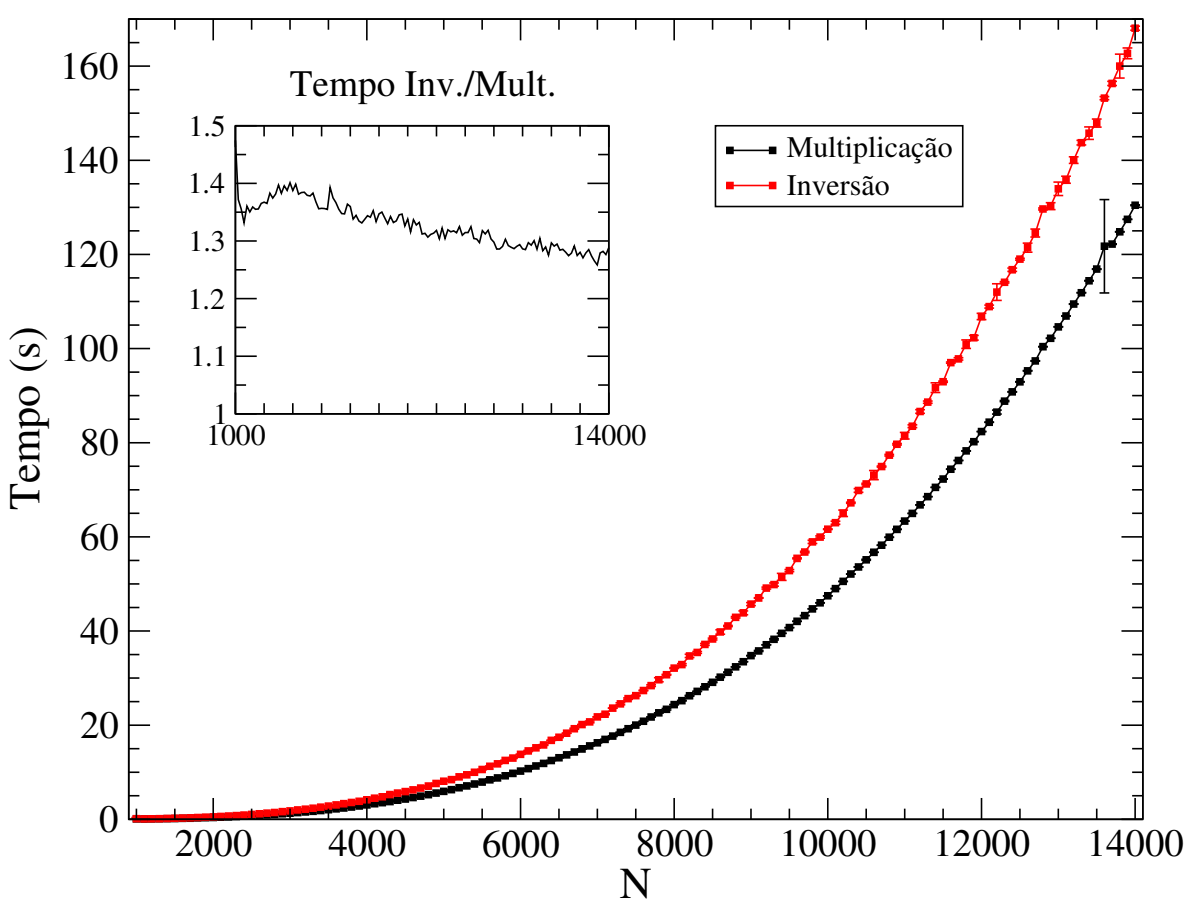

Figura D.1: Tempo de cálculo para multiplicação e inversão de matrizes de dimensão $N \times N$. Todos os cálculos foram feitos cinco vezes, as barras de erros mostram o desvio padrão. No detalhe, podemos ver a razão entre os tempos de inversão e multiplicação. Os cálculos foram realizados em paralelo (4 threads) em um processador Core-i7 960 usando as blibliotecas BLAS e LAPACK da Intel (MKL 11.1.059). 
mente $N=N_{1}+N_{2}$, podemos substituir $N_{1}=p N$ e $N_{2}=(1-p) N(\operatorname{com} 0<p<1)$ na Eq. (D.6), resultando em (depois de dividir por $N^{3}$ )

$$
\left[p^{3}+(1-p)^{3}+\frac{2 p(1-p)^{2}}{C_{\text {Inv }}}\right]+\frac{p^{2}}{C_{\text {Inv }} N} \leqslant 1
$$

Se $N$ for grande o suficiente, o último termo do lado direito na desigualdade acima, que é devido à soma na Eq. (D.5), pode ser desprezado:

$$
p^{3}+(1-p)^{3}+\frac{2 p(1-p)^{2}}{1.33} \leqslant 1
$$

Esta desigualdade é válida para todo o intervalo $0<p<1$ e apresenta um mínimo em torno de $p \sim 0.5$, ou seja, o método é sempre mais rápido que a inversão e apresenta o maior desempenho quando $N_{1} \approx N_{2} \approx N / 2$.

Na figura D.2 mostramos a performance relativa do método de decimação, em relação à inversão, para matrizes de dimensão $N$ e diversos tamanhos do bloco $A_{11}$, $M \equiv N_{1}$.

Outro aspecto importante a ser analisado é o erro numérico. Este foi calculado para todos os casos mostrados na fig. D.2 (em um total de 4194) como a diferença entre os valores absolutos dos elementos das inversas calculadas pelo método tradicional (através da fatoração LU) e pela decimação. Note que desta forma estamos calculando o erro em relação ao método tradicional, e não o erro absoluto. Os resultados obtidos podem ser vistos na fig. D.3. Como se pode ver, em nenhum caso o erro máximo foi maior que $10^{-5}$, o que garante que a decimação fornece um resultado "exato" (em comparação ao método tradicional) até a quinta casa decimal. 


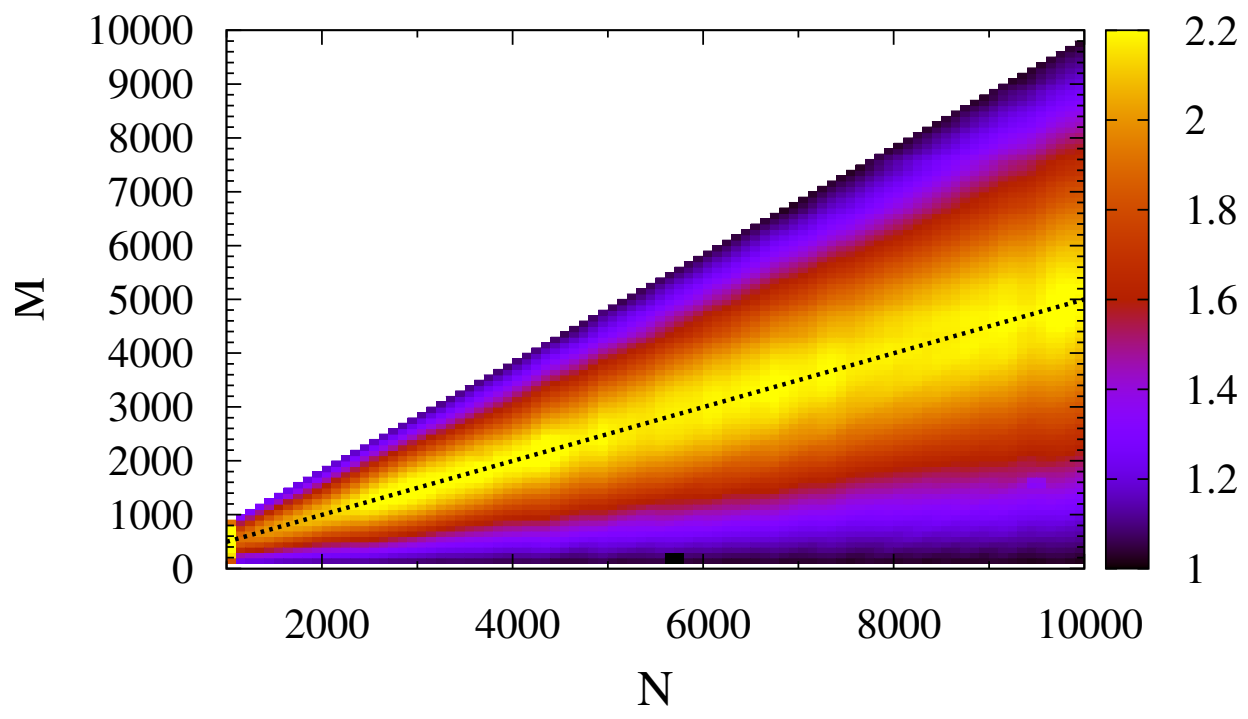

Figura D.2: Performance do método de decimação em relação à inversão para uma matriz de dimensão $N \times N$ da qual é decimado um bloco de dimensão $M \times M$. Um ganho de mais de $2 \times$ é obtido para $M \sim N / 2$ (linha pontilhada). Cálculos realizados em paralelo (4 threads) em um processador Core-i7 960 usando as blibliotecas BLAS e LAPACK da Intel (MKL 11.1.059). 

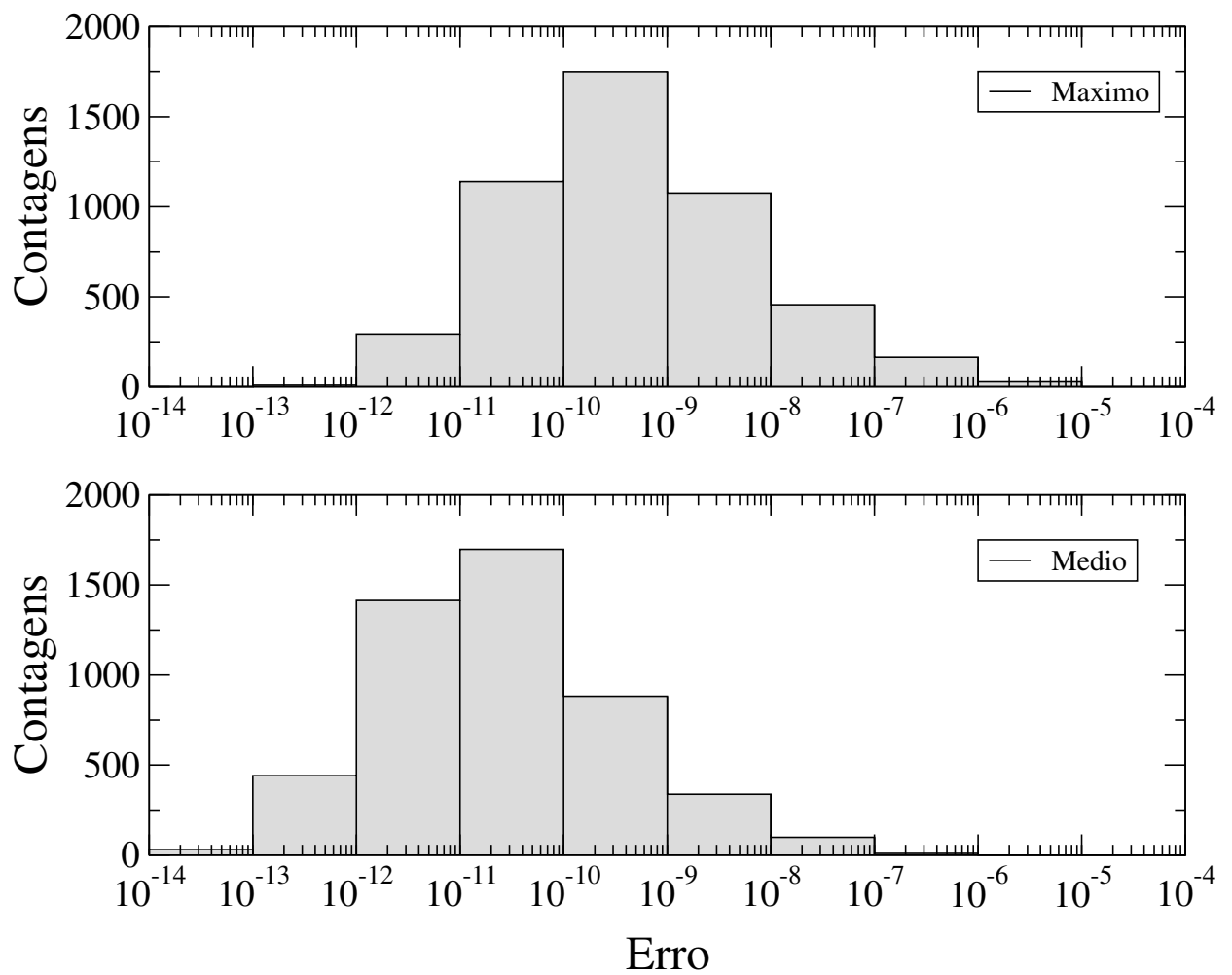

Figura D.3: Histograma dos erros máximo e médio por elemento do método de decimação em relação à inversão através da fatoração LU sobre um total de 4914 cálculos. Cálculos realizados usando as blibliotecas BLAS e LAPACK da Intel (MKL 11.1.059). 


\section{Generalizando para dimensão arbitrária}

Vamos generalizar o método acima para matrizes de dimensão (ou número de submatrizes) arbitrário. Novamente, temos o sistema de equações definido por $A B=1$ e que deve ser resolvido para determinar $B$. Este sistema, agora, é dado por

$$
\sum_{k=1}^{N} A_{i k} B_{k j}=\delta_{i j} \quad i, j=1, \ldots, N
$$

onde $N$ é a dimensão das matrizes $A$ e $B$. Suponhamos que $n \tilde{a} o$ estamos interessados em conhecer o elemento $B_{n m}$ da matriz $B$. Então, se separamos o termo $A_{i m} B_{m j}$ na somatória da Eq. (D.9),

$$
\sum_{k \neq m} A_{i k} B_{k j}+A_{i m} B_{m j}=\delta_{i j}
$$

podemos, de forma análoga ao caso anterior (Eq. D.4), reescrever $B_{m j}$, que está associado a termos que não queremos conhecer, em função de termos que nos interessam:

$$
B_{m j}=A_{i m}^{-1} \delta_{i j}-\sum_{k \neq m} A_{i k} B_{k j}
$$

Fixando $i=n$ na Eq. (D.11) temos,

$$
B_{m j}=A_{n m}^{-1} \delta_{n j}-\sum_{k \neq m} A_{n k} B_{k j}
$$

Substituindo o resultado acima em (D.10), como fizemos no caso anterior para determinar $B_{11}$ (Eq. D.5), obtemos

$$
\begin{gathered}
\sum_{k \neq m} A_{i k} B_{k j}+A_{i m}\left(A_{n m}^{-1} \delta_{n j}-\sum_{k \neq m} A_{n k} B_{k j}\right)=\delta_{i j} \\
\sum_{k \neq m}\left(A_{i k}-A_{i m} A_{n m}^{-1} A_{n k}\right) B_{k j}=\delta_{i j}-A_{i m} A_{n m}^{-1} \delta_{n j}
\end{gathered}
$$




$$
\sum_{k \neq m} \tilde{A}_{i k} B_{k j}=\delta_{i j}-A_{i m} A_{n m}^{-1} \delta_{n j},
$$

onde definimos

$$
\tilde{A}_{i k}=A_{i k}-A_{i m} A_{n m}^{-1} A_{n k} .
$$

Da Eq. (D.14), é fácil ver que, para a linha $i=n$ e para a coluna $k=m$, teremos $\tilde{A}_{n k}=0$ e $\tilde{A}_{i m}=0$. Isto significa que para a linha $n$ e a coluna $m$, que corresponde ao elemento que desejamos eliminar, a Eq. (D.15) é indeterminada, impossibilitando conhecer as respectivas linha e coluna da matriz $B$. Também, por conseguinte, o termo $A_{i m} A_{n m}^{-1} \delta_{n j}$ será nulo para os elementos nas linhas e colunas com $i \neq n$ e $j \neq m$.

Depois de todo este procedimento, o resultado final é que substituímos o sistema

$$
\left[\begin{array}{cccccc}
A_{11} & A_{12} & \cdots & A_{1 m} & \cdots & A_{1 N} \\
A_{21} & A_{22} & \cdots & A_{2 m} & \cdots & A_{2 N} \\
\vdots & \vdots & \ddots & \vdots & & \vdots \\
A_{n 1} & A_{n 2} & & A_{n m} & & A_{n N} \\
\vdots & \vdots & & \vdots & \ddots & \vdots \\
A_{N 1} & A_{N 2} & \cdots & A_{N m} & \cdots & A_{N N}
\end{array}\right]\left[\begin{array}{cccccc}
B_{11} & B_{12} & \cdots & B_{1 m} & \cdots & B_{1 N} \\
B_{21} & B_{22} & \cdots & B_{2 m} & \cdots & B_{2 N} \\
\vdots & \vdots & \ddots & \vdots & & \vdots \\
B_{n 1} & B_{n 2} & & B_{n m} & & B_{n N} \\
\vdots & \vdots & & \vdots & \ddots & \vdots \\
B_{N 1} & B_{N 2} & \cdots & B_{N m} & \cdots & B_{N N}
\end{array}\right]=1
$$


por um sistema equivalente, dado por

$$
\left[\begin{array}{cccccc}
\tilde{A}_{11} & \tilde{A}_{12} & \cdots & 0 & \cdots & \tilde{A}_{1 N} \\
\tilde{A}_{21} & \tilde{A}_{22} & \cdots & 0 & \cdots & \tilde{A}_{2 N} \\
\vdots & \vdots & \ddots & \vdots & & \vdots \\
0 & 0 & \cdots & 0 & \cdots & 0 \\
\vdots & \vdots & & \vdots & \ddots & \vdots \\
\tilde{A}_{N 1} & \tilde{A}_{N 2} & \cdots & 0 & \cdots & \tilde{A}_{N N}
\end{array}\right]\left[\begin{array}{cccccc}
B_{11} & B_{12} & \cdots & \mid & \cdots & B_{1 N} \\
B_{21} & B_{22} & \cdots & \mid & \cdots & B_{2 N} \\
\vdots & \vdots & \ddots & \mid & & \vdots \\
- & - & - & - & - & - \\
\vdots & \vdots & & \mid & \ddots & \vdots \\
B_{N 1} & B_{N 2} & \cdots & \mid & \cdots & B_{N N}
\end{array}\right]=1
$$

que efetivamente apresenta dimensão reduzida, pois a linha $i=n$ e a coluna $j=m$ foram "eliminadas", e que preserva o valor dos elementos da inversa que "interessam", $B_{i j}$ para $i \neq n$ e $j \neq m$.

\section{Especializando para matrizes tridiagonais}

Vamos, agora, tratar do caso específico de matrizes bloco-tridiagonais que são empregadas nos cálculos de desordem (cap. 7.1.3). Neste caso, queremos eliminar blocos da diagonal de uma matriz como a que segue:

$$
\left[\begin{array}{ccccc}
A_{11} & A_{12} & 0 & 0 & 0 \\
A_{21} & A_{22} & A_{23} & 0 & 0 \\
0 & A_{32} & A_{33} & A_{34} & 0 \\
0 & 0 & \ddots & \ddots & \ddots
\end{array}\right] .
$$

Neste caso, a expressão que define os elementos da matriz equivalente $\tilde{A}$ (Eq. D.16) é dada por

$$
\tilde{A}_{i j}=A_{i j}-A_{i n} A_{n n}^{-1} A_{n j}
$$


No entanto, como a matriz é tridiagonal, os elementos $A_{i n}$ e $A_{n j}$ são nulos se $i \neq n \pm 1$ ou $j \neq n \pm 1$. Ou seja,

$$
\begin{cases}\tilde{A}_{i j}=A_{i j}-A_{i n} A_{n n}^{-1} A_{n j} & i, j=n \pm 1 \\ \tilde{A}_{i j}=A_{i j} & i, j \neq n \pm 1\end{cases}
$$

e portanto, apenas os blocos adjacentes ao elemento eliminado sofrem alterações, todos os outros permanecendo iguais. Então se, por exemplo, eliminarmos o bloco $A_{22}$ da matriz acima (Eq. D.19), obteremos a matriz efetiva (descartando a linha e coluna eliminadas)

$$
\left[\begin{array}{cccc}
\tilde{A}_{11} & \tilde{A}_{13} & 0 & 0 \\
\tilde{A}_{31} & \tilde{A}_{33} & A_{34} & 0 \\
0 & \ddots & \ddots & \ddots
\end{array}\right] .
$$

É interessante notar que, para este caso este caso específico, o método pode ser aplicado recursivamente se desejado, eliminando um bloco por vez. 


\section{Apêndice E}

\section{Acelerando cálculos de transporte}

\section{usando CPUs e GPUs}

O contínuo e rápido aumento do poder computacional ocorrido nas últimas décadas, verificado na lei de Moore, permitiu um grande crescimento na área de simulação computacional de materiais (assim como em outras áreas). Atualmente é possível realizar cálculos ab initio de transporte eletrônico quântico em sistemas com centenas de átomos usando o método de funções de Green acoplado com DFT, por exemplo.

O advento das placas de processamento gráfico de propósito geral, GPGPUs ( General Purpose Graphical Processing Units), comumente chamadas apenas de GPUs, e da linguagem de programação CUDA (Compute Unified Device Architecture) [138] propiciou uma nova plataforma computacional com paralelismo massivo a baixo custo, pois GPUs possuem centenas de núcleos de processamento. Assim, o uso destes equipamentos pode acelerar significativamente códigos onde um alto grau de paralelismo seja possível. Isto ocorre, de forma geral, em problemas envolvendo álgebra linear, como multiplicação, diagonalização ou inversão, se as matrizes forem suficientemente grandes.

Para o cálculo em CPUs existem bibliotecas altamente otimizadas para resolver este tipo de problemas e que já se tornaram de uso padrão na computação de alta 
performance: a BLAS (Basic Linear Algebra Subprograms) [139] e LAPACK (Linear Algebra Package) [140]. Para GPUs, a Nvidia desenvolveu a CUBLAS (CUDA Basic Linear Algebra Subroutines) [141, distribuída gratuitamente, que é uma versão para GPU da BLAS. Grande parte da LAPACK foi portada para GPU na biblioteca MAGMA (Matrix Algebra on GPU and Multicore Architectures) [142], desenvolvida pelo mesmo criador da BLAS e LAPACK e distribuida gratuitamente.

Neste trabalho, implementamos o uso de GPUs no código TRANSAMPA [14, 15] através da bibliotecas CUBLAS e MAGMA e investigamos a performance e precisão obtidas. Todos os testes mostrados aqui foram realizados em computadores munidos de dois processadores (CPUs) Intel Xeon X5570 (com 4 núcleos cada) e duas GPUs Nvidia Tesla M2050 (com 448 núcleos cada).

\section{Revisão Teórica}

No formalismo de Landauer-Büttiker a corrente de um dispositivo de duas pontas é dado por (Eq. 2.13)

$$
I_{\sigma}=\frac{e}{h} \int \mathcal{T}_{\sigma}(E)\left(f_{L}-f_{R}\right) d E
$$

onde $\sigma$ é o spin, e é carga do elétron, $h$ é a constante de Planck, $\mathcal{T}_{\sigma}(E)$ é a função de transmissão, $f_{L(R)} \equiv f\left(E, \mu_{L(R)}, T_{L(R)}\right)$ é a função de distribuição de Fermi-Dirac, $\mu_{L(R)}$ e $T_{L(R)}$ são o potenciais químicos e a temperatura dos contatos esquerdo (L) e direito $(\mathrm{R})$, respectivamente.

No regime linear, expressões para os coeficientes de transporte como as condutâncias eletrônica e térmica eletrônica, $G$ e $K$ respectivamente, e o coeficiente Seebeck, $S$, podem ser derivados de expansões da Eq. (E.1) (a partir de agora, exceto quando 
necessário, omitiremos o subscrito $\sigma)$ :

$$
\begin{aligned}
G & =\frac{e^{2}}{h} \mathcal{K}_{0} \\
K & =\frac{1}{h T}\left(\mathcal{K}_{2}-\frac{\mathcal{K}_{1}^{2}}{\mathcal{K}_{0}}\right) \\
S & =-\frac{1}{e T} \frac{\mathcal{K}_{1}}{\mathcal{K}_{0}},
\end{aligned}
$$

onde $T$ é a temperatura média entre os eletrodos e $\mathcal{K}_{n}$ é definido como

$$
\mathcal{K}_{n}=\int \mathcal{T}(E)\left(-\frac{\partial f}{\partial E}\right)(E-\mu)^{n} d E
$$

Vemos então que a transmitância é o objeto central deste formalismo. Uma vez que $\mathcal{T}(E)$ é conhecida, o cálculo das eqs E.2 E.4 é imediato.

No código TRANSAMPA o cálculo da função de transmitância é realizado acoplando a teoria do funcional da densidade, via o código SIESTA [70, 71], com o formalismo de funções de Green fora do equilíbrio (NEGF+DFT) de forma autoconsistente . Neste formalismo a estrutura do sistema é (Eq. 2.16)

$$
H=\left[\begin{array}{ccc}
H_{L} & H_{L C} & \\
H_{C L} & H_{C} & H_{C R} \\
& H_{R C} & H_{C R}
\end{array}\right]
$$

e a transmitância é dada por

$$
\begin{aligned}
\mathcal{T}(E) & =\sum_{k} \mathcal{T}(E, k) \\
& =\sum_{k} \operatorname{Tr}\left\{\Gamma_{L}^{k}(E) G_{a}^{k}(E) \Gamma_{R}^{k}(E) G_{r}^{k}(E)\right\}
\end{aligned}
$$

onde as matrizes $\Gamma_{L(R)}$ descrevem o acoplamento da região central de espalhamento 
com os eletrodos esquerdo (direito) e são dadas por

$$
\Gamma_{L(R)}^{k}(E)=i\left[\Sigma_{L(R)}^{k}(E)-\Sigma_{L(R)}^{k}(E)^{\dagger}\right]
$$

$G_{r}^{k}$ é a função de Green retardada da região central, definida como

$$
G_{r}^{k}(E)=\left[H_{C}^{k}+\Sigma_{L}^{k}(E)+\Sigma_{R}^{k}(E)-E S_{C}^{k}\right]^{-1}
$$

e $\Sigma_{L(R)}$ é a auto-energia dos eletrodos, dada por

$$
\Sigma_{L(R)}^{k}(E)=-H_{C L(R)}^{k} g_{L(R)}^{k}(E) H_{L(R) C}^{k}
$$

Note que apesar da função de Green dos eletrodos, $g_{L(R)}^{k}$, e as hamiltonianas de acoplamento da região central com o eletrodos, $H_{C L(R)}^{k}$ e $H_{L(R) C}^{k}$, serem matrizes semi-infinitas, devido à ordem das multiplicações na Eq. (E.11) $\Sigma_{L(R)}^{k}$ é finita.

Fica então a questão de como calcular a função de Green dos eletrodos. Para abordar esta pergunta vamos considerar a Eq. (E.11) acima para o eletrodo esquerdo, $\Sigma_{L}=-H_{C L} g_{L} H_{L C}$ (na qual simplificamos a notação), onde a hamiltoniana do eletrodo esquerdo é

$$
H_{L}=\left[\begin{array}{cccc}
\ddots & \ddots & & \\
\ddots & H_{0} & H_{1} & \\
& H_{1}^{\dagger} & H_{0} & H_{1} \\
& & H_{1}^{\dagger} & H_{0}
\end{array}\right]
$$

as hamiltonianas de acoplamento com a região central são

$$
H_{C L}=\left[\begin{array}{ccc}
\cdots & 0 & H_{1}^{\dagger} \\
& 0 & 0 \\
\cdot & & \vdots
\end{array}\right]
$$


e $H_{L C}=H_{C L}^{\dagger}$, e a função de Green do eletrodo é

$$
g_{L}=\left(H_{L}-E S_{L}\right)^{-1}
$$

Assim, vemos que, dada a estrutura interna de $H_{L}$ e $H_{C L}$, só é preciso conhecer o bloco inferior direito (com a mesma dimensão de $H_{0}$ ) da função de Green do eletrodo. Esta submatriz de $g_{L}$, a única que não se anulará ao realizar as multiplicações na Eq. E.11), é conhecida como função de Green de superfície, $g_{s L}$, e pode ser calculada usando o método de decimação descrito no apêndice D. No entanto, dado que os blocos que compõem as diagonais de $H_{L}$ são iguais é possível renormalizar a cada interação o bloco inferior direito (no caso do eletrodo esquerdo) correspondente a $g_{s L}$ e metade da matriz, renormalizando $H_{0}$ e $H_{1}$ a cada passo. Usado desta forma o método é chamado de método de renormalização $2^{n}$ [143].

\section{Implementação e Resultados}

Na fig. E.1 está representado um fluxograma mostrando as etapas de um cálculo de transporte NEGF + DFT de forma simplificada. As etapas nas quais se fazem cálculos sobre várias energias e pontos $k$ (loops sobre $E$ e $k$ ) que são independentes estão indicados pelas caixas cinzas. Estas são a convergência autoconsistente da matriz de densidade $(D M)$, que é calculada por uma integral de contorno através do teorema dos resíduos [144] e o cálculo da função de transmitância sendo calculada repetidas vezes para várias energias e pontos $k$. Fica claro então que a evaluação da função de Green de superfície, $g_{s}$, é central nos cálculos de transporte e por ser dominada por multiplicação e inversão de matrizes oferece uma oportunidade para o uso eficiente e fácil de GPUs através das bibliotecas CUBLAS e MAGMA. De forma geral, nos cálculos mostrados nesta tese, o tempo gasto no cálculo da função de Green de superfície foi de aproximadamente $90 \%$ do total. 


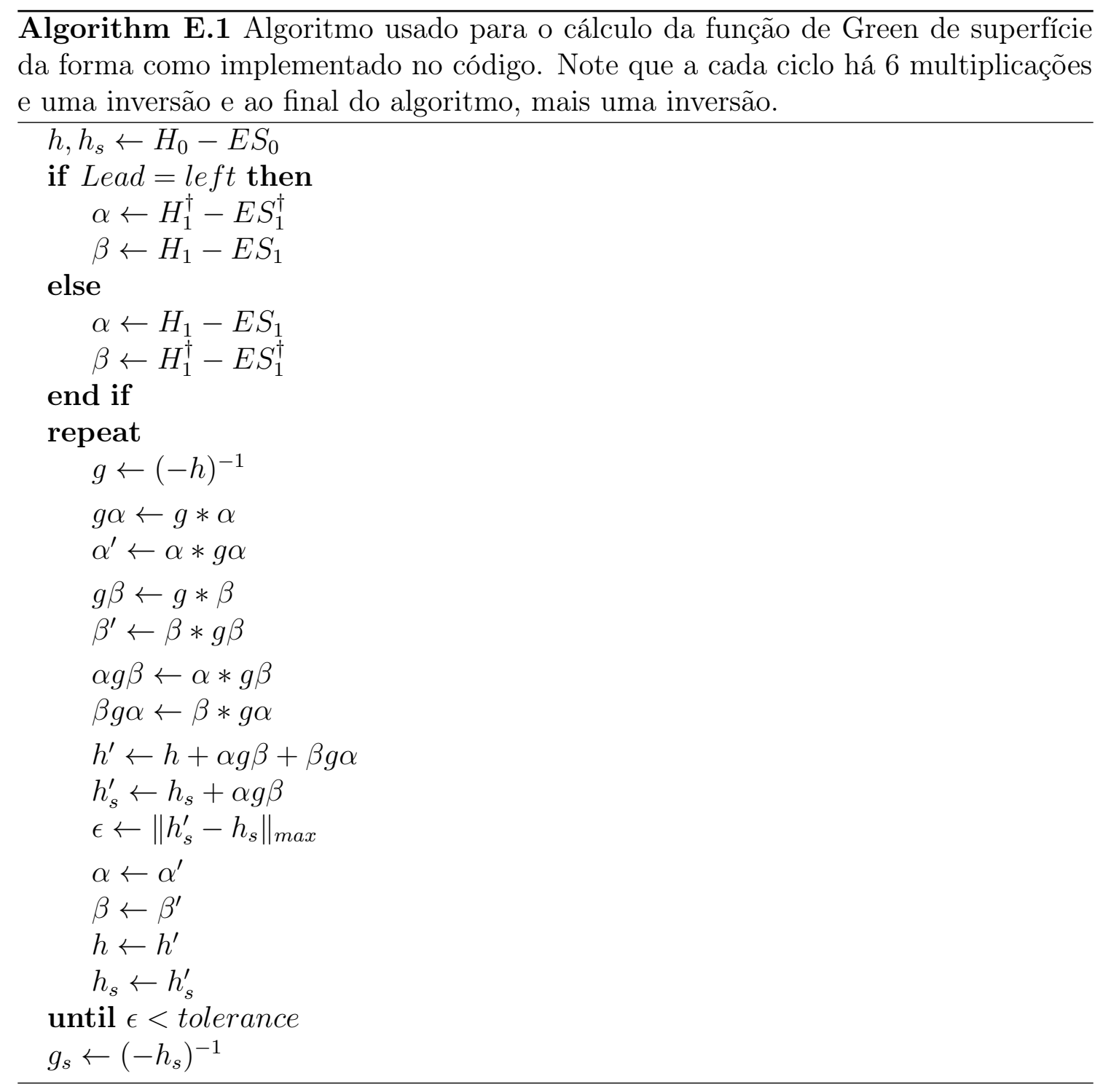




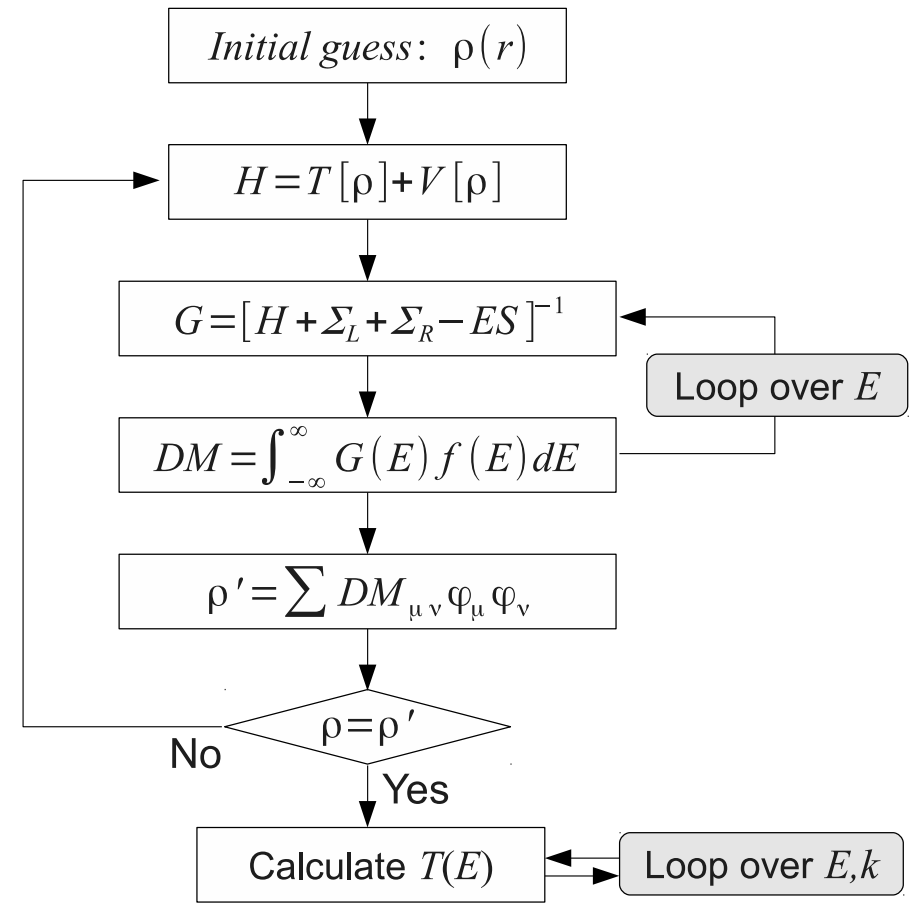

Figura E.1: Fluxograma do código de cálculo de transporte eletrônico NEGF+DFT.

O algoritmo implementado pelo autor no código TRANSAMPA para cálcular $g_{s}$ é mostrado em Alg. E.1. No código, as multiplicações (6/ciclo) e inversões (1/ciclo + 1) de matrizes são realizadas através de chamadas a funções das bibliotecas BLAS e LAPACK quando o cálculo é realizado em uma CPU, e para as bibliotecas CUBLAS e MAGMA quando o cálculo é feito em uma GPU.

Para obter um balanço de carga ideal entre o trabalho realizado por CPUs e GPUs e evitar que muito trabalho seja destinado as CPUs, deixando as GPUs ociosas, ou vice-versa, foi implementada a distribuição dinâmica dos pontos dos grids de energia nos quais a $D M$ e $\mathcal{T}(E)$ são calculados (os loops de energia indicados na fig. E.1) segundo o esquema indicado na fig. E.2. $M$ dos $N$ processos trabalhadores se ligam as GPUs disponíveis (pode ser mais de um processo por GPU) e utilizam as bibliotecas CUBLAS/MAGMA. Os $N-M$ processos restantes procedem com chamadas às bibliotecas BLAS/LAPACK. O processo mestre distribui as energias sob requisição dos processos trabalhadores.

A implementação foi testada no mesmo sistema apresentado na seção 5, um 


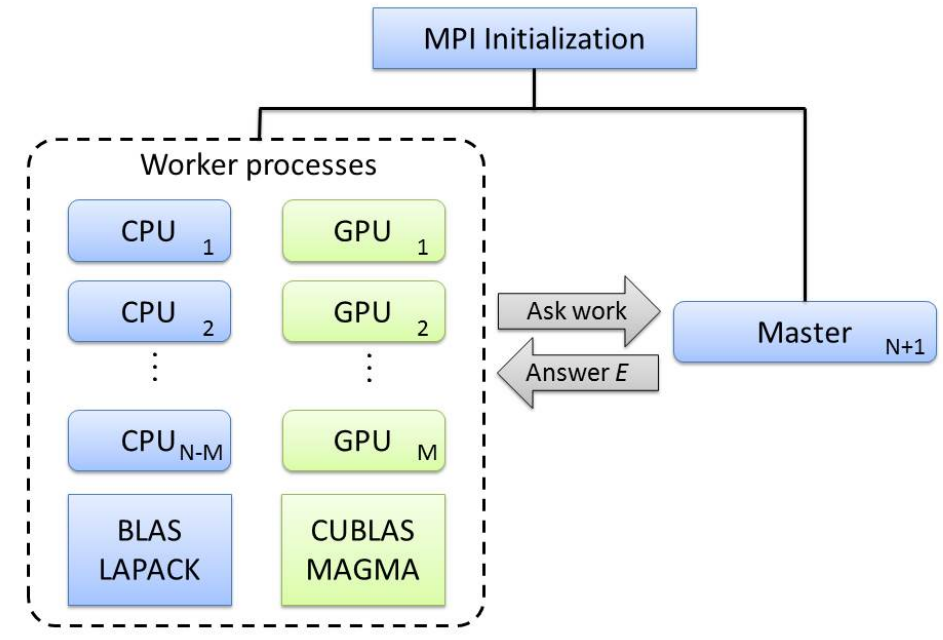

Figura E.2: Representação esquemática da abordagem usada para obter um bom balanço de carga entre as CPUs e as GPUs. $M$ processos se ligam a GPUs (pode ser mais de um processo por GPU) e realizam multiplicações e inversões de matrizes através de chamadas às bibliotecas CUBLAS/MAGMA. Os $N-M$ processos restantes procedem com chamadas às bibliotecas BLAS/LAPACK. O trabalho (pontos dos grids de energia) é distribuído dinamicamente pelo processo mestre sob requisição.

dispositivo de grafeno com um átomo de Mn preso em uma monovacância (sem aplicação de potencial de gate, $\left.V_{g}=0\right)$. Nos resultados, apresentados na fig. E.3. podemos ver que as transmitâncias para os canais de spin up e down calculados apenas em CPUs ou apenas em GPUs são indistinguíveis a olho nu. O erro relativo, tomando o calculo em CPU como referência, $\left|\mathcal{T}_{G P U}-\mathcal{T}_{C P U}\right| / \mathcal{T}_{C P U}$, no intervalo de energias calculadas é sempre menor que $10^{-4}$, sendo que a maioria dos erros ( $\left.90 \%\right)$ é menor que $10^{-5}$. Assim, vemos que a precisão no cálculo com GPUs é mantido e que os resultados são virtualmente idênticos aos obtidos com CPUs.

O tempo de execução do código e a aceleração obtida em função no número de processos ligados a CPUs e GPUs (lembre que os computadores usados possuem 2 CPUs com 4 núcleos cada e 2 GPUs) são mostradas na figura E.4 na qual podemos ver que o uso de GPUs proporciona uma aceleração significativa dos cálculos. Quando um processo é ligado a cada GPU, o tempo de execução do programa é reduzido em $58 \%$, passando de $50 \mathrm{~h}$ para $21 \mathrm{~h} 9 \mathrm{~m}$. A medida que mais processos pas- 

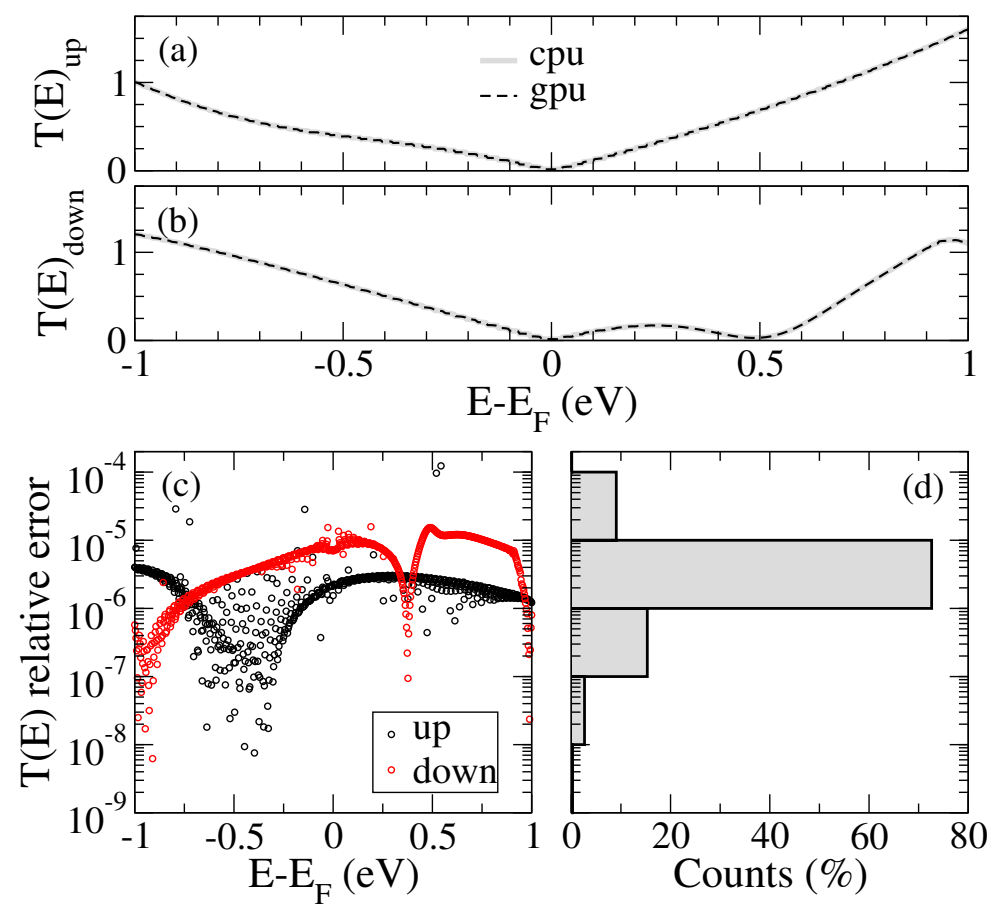

Figura E.3: Transmitância para os canais de spin up (a) e down (b) calculados usando apenas CPUs (linha cinza) e apenas GPUs (linha preta tracejada). (b) Erro relativo na transmitância, $\left|\mathcal{T}_{\text {gpu }}-\mathcal{T}_{\text {cpu }}\right| / \mathcal{T}_{\text {cpu }}$. (d) Histograma das magnitudes do erro relativo. 


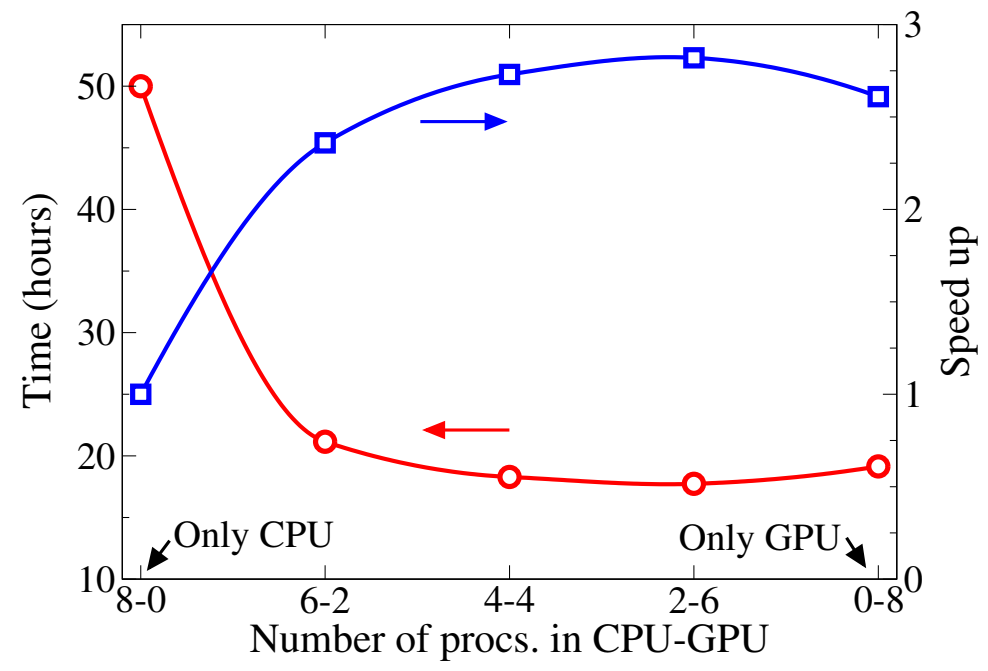

Figura E.4: Tempo de execução (círculos vermelhos) e aceleração (quadrados azuis) versus número de processos ligados CPUs e GPUs. As linhas são apenas guias para a visão.

sam a se ligar às duas GPUs o tempo de cálculo vai diminuindo até atingir o mínimo de $17 \mathrm{~h} 44 \mathrm{~m}$, com uma diminuição de $65 \%$ (aceleração de 2.82 vezes) em relação ao cálculo sem uso de GPUs, quando há 3 processos ligados a cada GPU. Depois disso, as GPUs começam a ficar sobrecarregadas, com um aumento no tempo execução, 19h $9 \mathrm{~m}$.

Em suma, vemos que a implementação do uso de GPUs no código TRANSAMPA resultou em uma aceleração de quase 3 vezes enquanto a precisão é mantida. Vale lembrar que esta implementação foi feita de forma simples, da forma mais transparente possível no programa. Assim, há mais espaço para melhorias no código, como por exemplo o uso de áreas de memória restritas (pinned memory) para a transferência dos dados entre a CPU e a GPU, por exemplo, que podem melhorar ainda mais a performance do programa. 


\section{Referências Bibliográficas}

[1] GEIM, A. K.; NOVOSELOV, K. S. The rise of graphene. Nature materials, v. 6, n. 3, p. 183-91, Mar. 2007.

[2] LI, X.; WANG, X.; ZHANG, L.; LEE, S.; DAI, H. Chemically derived, ultrasmooth graphene nanoribbon semiconductors. Science, Washington, v. 319, n. 5867, p. 1229-32, Feb. 2008.

[3] SON, Y.-W.; COHEN, M. L.; LOUIE, S. G. Half-metallic graphene nanoribbons. Nature, London, v. 444, n. 7117, p. 347-9, Nov. 2006.

[4] BAughman, R. H.; ZAKhidov, A. A.; DE HEER, W. A. Carbon nanotubes-the route toward applications. Science, Washington, v. 297, n. 5582, p. 787-92, Aug. 2002.

[5] DRESSElHAUS, M. S.; LIN, Y.-M.; RABIN, O.; BLACK, M. R.; DRESSELHAUS, G. Nanowires. In: BHUSHAN, B. (Ed.) Springer Handbook of Nanotechnology. Springer, 2010. p. 119-165.

[6] ASHCROFT, N. W.; MERMIN, N. D. Solid State Physics. ITPS Thomson Learning, 2000.

[7] LUNDSTROM, M. Fundamentals of carrier transport. Cambridge University Press, 2000. 
[8] BELL, L. E. Cooling, heating, generating power, and recovering waste heat with thermoelectric systems. Science, Washington, v. 321, n. 5895, p. 1457-61, Sept. 2008.

[9] DISALVO, F. J. Thermoelectric Cooling and Power Generation. Science, Washington, v. 285, n. 5428, p. 703-706, July 1999.

[10] MAHAN, G.; SALES, B.; SHARP, J. Thermoelectric Materials: New Approaches to an Old Problem. Physics Today, v. 50, n. 3, p. 42, 1997.

[11] HARMAN, T. C.; TAYLOR, P. J.; WALSH, M. P.; LAFORGE, B. E. Quantum dot superlattice thermoelectric materials and devices. Science, Washington, v. 297, n. 5590, p. 2229-32, Sept. 2002.

[12] MAHAN, G. D.; SOFO, J. O. The best thermoelectric. Proceedings of the National Academy of Sciences, v. 93, p. 7436, 1996.

[13] REDDY, P.; JANG, S.-Y.; SEGALMAN, R. A.; MAJUMDAR, A. Thermoelectricity in molecular junctions. Science, Washington, v. 315, n. 5818, p. 1568-71, Mar. 2007.

[14] NOVAES, F. D.; DA SILVA, A. J. R.; FAZZIO, A. Density functional theory method for non-equilibrium charge transport calculations: TRANSAMPA. Brazilian Journal of Physics, São Paulo, v. 36, n. 3a, p. 799-807, Sept. 2006.

[15] PADILHA, J. E.; LIMA, M. P.; DA SILVA, A. J. R.; FAZZIO, A. Bilayer graphene dual-gate nanodevice: An ab initio simulation. Physical Review B, v. 84 , n. 11, p. 113412, Sept. 2011.

[16] KROTO, H. W.; HEATH, J. R.; O’BRIEN, S. C.; CURL, R. F.; SMALlEY, R. E. C60: Buckminsterfullerene. Nature, London, v. 318, n. 6042, p. 162-163, Nov. 1985. 
[17] IIJIMA, S. Helical microtubules of graphitic carbon. Nature, London, v. 354, n. 6348, p. 56-58, Nov. 1991.

[18] WALlaCE, P. The Band Theory of Graphite. Physical Review, New York, v. 71, n. 9, p. 622-634, May 1947.

[19] NOVOSELOV, K. S.; GEIM, A. K.; MOROZOV, S. V.; JIANG, D.; ZHANG, Y.; DubOnOS, S. V.; GRIGORIEVA, I. V.; FIRSOV, A. A. Electric field effect in atomically thin carbon films. Science, Washington, v. 306, n. 5696, p. 666-9, Oct. 2004.

[20] MOROZOV, S.; NOVOSElOV, K.; KATSNElSON, M.; SCHEDIN, F.; ELIAS, D.; JASZCZAK, J.; GEIM, A. Giant Intrinsic Carrier Mobilities in Graphene and Its Bilayer. Physical Review Letters, Woodbury, v. 100, n. 1, p. 016602, Jan. 2008.

[21] LEE, C.; WEI, X.; KYSAR, J. W.; HONE, J. Measurement of the elastic properties and intrinsic strength of monolayer graphene. Science, Washington, v. 321, n. 5887, p. 385-8, July 2008.

[22] BALANDIN, A. A.; GHOSH, S.; BAO, W.; CALIZO, I.; TEWELDEBRHAN, D.; MIAO, F.; LAU, C. N. Superior thermal conductivity of single-layer graphene. Nano letters, v. 8, n. 3, p. 902-7, Mar. 2008.

[23] Castro Neto, A. H.; PERES, N. M. R.; NOVOSElOV, K. S.; GEIM, A. K. The electronic properties of graphene. Reviews of Modern Physics, Woodbury, v. 81, n. 1, p. 109-162, Jan. 2009.

[24] ZUEV, Y.; CHANG, W.; KIM, P. Thermoelectric and Magnetothermoelectric Transport Measurements of Graphene. Physical Review Letters, Woodbury, v. 102, n. 9, p. 096807, Mar. 2009. 
[25] WEI, P.; BAO, W.; PU, Y.; LAU, C.; SHI, J. Anomalous Thermoelectric Transport of Dirac Particles in Graphene. Physical Review Letters, Woodbury, v. 102, n. 16, p. 166808, Apr. 2009.

[26] CHECKELSKY, J.; ONG, N. Thermopower and Nernst effect in graphene in a magnetic field. Physical Review B, v. 80, n. 8, p. 081413, Aug. 2009.

[27] SIDOROV, A. N.; SHEREHIY, A.; JAYASINGHE, R.; STALLARD, R.; BENJAMIN, D. K.; YU, Q.; LIU, Z.; WU, W.; CAO, H.; CHEN, Y. P.; JIANG, Z.; SUMANASEKERA, G. U. Thermoelectric power of graphene as surface charge doping indicator. Applied Physics Letters, Woodbury, v. 99, n. 1, p. 013115, 2011.

[28] NAKADA, K.; FUJITA, M.; DRESSELHAUS, G.; DRESSELHAUS, M. Edge state in graphene ribbons: Nanometer size effect and edge shape dependence. Physical Review B, v. 54, n. 24, p. 17954-17961, Dec. 1996.

[29] MARTIN, R. Electronic Structure: basic theory and practical methods. Cambridge University Press, 2004.

[30] PISANI, L.; CHAN, J.; MONTANARI, B.; HARRISON, N. Electronic structure and magnetic properties of graphitic ribbons. Physical Review B, v. 75, n. 6, p. 064418, Feb. 2007.

[31] KOBAYASHI, Y.; FUKUI, K.-I.; ENOKI, T.; KUSAKABE, K.; KABURAGI, Y. Observation of zigzag and armchair edges of graphite using scanning tunneling microscopy and spectroscopy. Physical Review B, v. 71, n. 19, p. 193406, May 2005.

[32] KOBAYASHI, Y.; FUKUI, K.-I.; ENOKI, T. Edge state on hydrogenterminated graphite edges investigated by scanning tunneling microscopy. Physical Review B, v. 73, n. 12, p. 125415, Mar. 2006. 
[33] LEMME, M. C.; ECHTERMEYER, T. J.; BAUS, M.; KURZ, H. A Graphene Field-Effect Device. IEEE Electron Device Letters, v. 28, n. 4, p. 282-284, Apr. 2007.

[34] CHEN, Z.; LIN, Y.-M.; ROOKS, M. J.; AVOURIS, P. Graphene nano-ribbon electronics. Physica E: Low-dimensional Systems and Nanostructures, v. 40, n. 2, p. 228-232, Dec. 2007.

[35] STAMPFER, C.; SCHURTENBERGER, E.; MOLITOR, F.; GüTTINGER, J.; IHN, T.; ENSSLIN, K. Tunable graphene single electron transistor. Nano letters, v. 8, n. 8, p. 2378-83, Aug. 2008.

[36] PONOMARENKO, L. A.; SCHEDIN, F.; KATSNELSON, M. I.; YANG, R.; HILl, E. W.; NOvOSELOV, K. S.; GEIM, A. K. Chaotic Dirac billiard in graphene quantum dots. Science, Washington, v. 320, n. 5874, p. 356-8, Apr. 2008.

[37] WANG, Z. F.; SHI, Q. W.; LI, Q.; WANG, X.; HOU, J. G.; ZHENG, H.; YAO, Y.; CHEN, J. Z-shaped graphene nanoribbon quantum dot device. Applied Physics Letters, Woodbury, v. 91, n. 5, p. 053109, 2007.

[38] STANDlEY, B.; BAO, W.; ZHANG, H.; BRUCK, J.; LAU, C. N.; BOCKRATH, M. Graphene-based atomic-scale switches. Nano letters, v. 8, n. 10, p. $3345-9$, Oct. 2008.

[39] ECHTERMEYER, T.; LEMME, M.; BAUS, M.; SZAFRANEK, B.; GEIM, A.; KURZ, H. Nonvolatile Switching in Graphene Field-Effect Devices. IEEE Electron Device Letters, v. 29, n. 8, p. 952-954, Aug. 2008.

[40] SCHEDIN, F.; GEIM, A. K.; MOROZOV, S. V.; HILL, E. W.; BLAKE, P.; KATSNELSON, M. I.; NOVOSELOV, K. S. Detection of individual gas molecules adsorbed on graphene. Nat. Mater., v. 6, n. 9, p. 652-655, 2007. 
[41] ROBInSON, J. T.; PERKINS, F. K.; SNOW, E. S.; WEI, Z.; SHEEHAN, P. E. Reduced graphene oxide molecular sensors. Nano letters, v. 8, n. 10, p. 3137-40, Oct. 2008.

[42] HWANG, E. H.; ADAM, S.; Das Sarma, S. Transport in chemically doped graphene in the presence of adsorbed molecules. Physical Review B, v. 76, n. 19, p. 195421, Nov. 2007.

[43] WANG, X.; ZHI, L.; MüLLEN, K. Transparent, conductive graphene electrodes for dye-sensitized solar cells. Nano letters, v. 8, n. 1, p. 323-7, Jan. 2008.

[44] LI, D.; KANER, R. B. Materials science. Graphene-based materials. Science, Washington, v. 320, n. 5880, p. 1170-1, May 2008.

[45] OHISHI, M.; SHIRAISHI, M.; NOUCHI, R.; NOZAKI, T.; SHINJO, T.; SUZUKI, Y. Spin Injection into a Graphene Thin Film at Room Temperature. Japanese Journal of Applied Physics, v. 46, n. No. 25, p. L605-L607, June 2007.

[46] JóZSA, C.; POPINCIUC, M.; TOMBROS, N.; JONKMAN, H. T.; VAN WEES, B. J. Electronic Spin Drift in Graphene Field-Effect Transistors. Physical Review Letters, Woodbury, v. 100, n. 23, p. 236603, June 2008.

[47] HILL, E.; GEIM, A.; NOVOSELOV, K.; SCHEDIN, F.; BLAKE, P. Graphene Spin Valve Devices. IEEE Transactions on Magnetics, New York, v. 42, n. 10, p. 2694-2696, Oct. 2006.

[48] CHO, S.; CHEN, Y.-F.; FUHRER, M. S. Gate-tunable graphene spin valve. Applied Physics Letters, Woodbury, v. 91, n. 12, p. 123105, 2007.

[49] TRAUZETTEL, B.; BULAEV, D. V.; LOSS, D.; BURKARD, G. Spin qubits in graphene quantum dots. Nature Physics, v. 3, n. 3, p. 192-196, Feb. 2007. 
[50] PEDERSEN, T.; FLINDT, C.; PEDERSEN, J.; MORTENSEN, N.; JAUHO, A.-P.; PEDERSEN, K. Graphene Antidot Lattices: Designed Defects and Spin Qubits. Physical Review Letters, Woodbury, v. 100, n. 13, p. 136804, Apr. 2008.

[51] TAPASZTó, L.; DOBRIK, G.; LAMBIN, P.; BIRó, L. P. Tailoring the atomic structure of graphene nanoribbons by scanning tunnelling microscope lithography. Nature nanotechnology, v. 3, n. 7, p. 397-401, July 2008.

[52] ZHANG, Y.; TANG, T.-T.; GIRIT, C.; HAO, Z.; MARTIN, M. C.; ZETTL, A.; CROMmIE, M. F.; SHEN, Y. R.; WANG, F. Direct observation of a widely tunable bandgap in bilayer graphene. Nature, London, v. 459, n. 7248, p. 820-3, June 2009.

[53] WANG, C.-R.; LU, W.-S.; HAO, L.; LEE, W.-L.; LEE, T.-K.; LIN, F.; CHENG, I.-C.; CHEN, J.-Z. Enhanced Thermoelectric Power in Dual-Gated Bilayer Graphene. Physical Review Letters, Woodbury, v. 107, n. 18, p. 186602, Oct. 2011.

[54] NAM, S.-G.; KI, D.-K.; LEE, H.-J. Thermoelectric transport of massive Dirac fermions in bilayer graphene. Physical Review B, v. 82, n. 24, p. 245416, Dec. 2010.

[55] ARADHYA, S. V.; VENKATARAMAN, L. Single-molecule junctions beyond electronic transport. Nature nanotechnology, v. 8, n. 6, p. 399-410, June 2013.

[56] REED, M. A. Conductance of a Molecular Junction. Science, Washington, v. 278, n. 5336, p. 252-254, Oct. 1997.

[57] PONTES, R. B.; ROCHA, A. R.; SANVITO, S.; FAZZIO, A.; DA SILVA, A. J. R. Ab initio calculations of structural evolution and conductance of benzene-1,4-dithiol on gold leads. ACS nano, v. 5, n. 2, p. 795-804, Feb. 2011. 
[58] LANDAUER, R. Electrical resistance of disordered one-dimensional lattices. Philosophical Magazine, v. 21, n. 172, p. 863-867, Apr. 1970.

[59] Di Ventra, M. Electrical Transport in Nanoscale Systems. Cambridge University Press, 2008.

[60] DATTA, S. Electronic Transport in Mesoscopic Physics. Cambridge University Press, 1995.

[61] REGO, L.; KIRCZENOW, G. Quantized Thermal Conductance of Dielectric Quantum Wires. Physical Review Letters, Woodbury, v. 81, n. 1, p. 232-235, July 1998.

[62] WANG, J.-S.; WANG, J.; Lü, J. T. Quantum thermal transport in nanostructures. The European Physical Journal B, Les Ulis, v. 62, n. 4, p. 381-404, May 2008.

[63] JIANG, J.-W.; WANG, J.-S.; LI, B. A nonequilibrium Green's function study of thermoelectric properties in single-walled carbon nanotubes. Journal of Applied Physics, Woodbury, v. 109, n. 1, p. 014326, 2011.

[64] CHOWDHURY, I.; PRASHER, R.; LOFGREEN, K.; CHRYSLER, G.; NARASIMHAN, S.; MAHAJAN, R.; KOESTER, D.; ALLEY, R.; VENKATASUBRAMANIAN, R. On-chip cooling by superlattice-based thin-film thermoelectrics. Nature nanotechnology, v. 4, n. 4, p. 235-8, Apr. 2009.

[65] PAULSSON, M.; DATTA, S. Thermoelectric effect in molecular electronics. Physical Review B, v. 67, n. 24, p. 241403, June 2003.

[66] LUNDE, A. M.; FLENSBERG, K. On the Mott formula for the thermopower of non-interacting electrons in quantum point contacts. Journal of physics. Condensed matter : an Institute of Physics journal, v. 17, n. 25, p. 3879-84, June 2005. 
[67] BERGFIELD, J. P.; STAFFORD, C. A. Thermoelectric signatures of coherent transport in single-molecule heterojunctions. Nano letters, v. 9, n. 8, p. 30726, Aug. 2009.

[68] ROWE, D. M. General Principles and Basic Considerations. In: ROWE, D. M. (Ed.) Thermoelectrics Handbook: Macro to Nano. CRC Press, 2005. p. $1-14$.

[69] PRESS, W.; TEUKOLSKY, S.; VETTERLING, W.; FlAnNERY, B. Numerical Recipes in C. 2nd. ed. Cambridge, UK: Cambridge University Press, 1992.

[70] ARTACHO, E.; Sï¡œNCHEZ-PORTAL, D.; ORDEJï¡œN, P.; GARCï¿œA, A.; SOLER, J. Linear-Scaling ab-initio Calculations for Large and Complex Systems. physica status solidi (b), v. 215, n. 1, p. 809-817, Sept. 1999.

[71] SOLER, J. M.; ARTACHO, E.; GALE, J. D.; GARCÍA, A.; JUNQUERA, J.; ORDEJóN, P.; SáNCHEZ-PORTAL, D. The SIESTA method for ab initio order- N materials simulation. Journal of Physics: Condensed Matter, Bristol, v. 14, n. 11, p. 2745-2779, Mar. 2002.

[72] MARTINS, T.; MIWA, R.; DA SILVA, A.; FAZZIO, A. Electronic and Transport Properties of Boron-Doped Graphene Nanoribbons. Physical Review Letters, Woodbury, v. 98, n. 19, p. 196803, May 2007.

[73] ZENG, M.; FENG, Y.; LIANG, G. Graphene-based spin caloritronics. Nano letters, v. 11, n. 3, p. 1369-73, Mar. 2011.

[74] LIMA, M. P.; DA SILVA, A. J. R.; FAZZIO, A. Adatoms in graphene as a source of current polarization: Role of the local magnetic moment. Physical Review B, v. 84, n. 24, p. 245411, Dec. 2011. 
[75] TROULLIER, N.; MARTINS, J. L. Efficient pseudopotentials for plane-wave calculations. Physical Review B, v. 43, n. 3, p. 1993-2006, Jan. 1991.

[76] LIMA, M. P.; DA SILVA, A. J. R.; FAZZIO, A. Splitting of the zero-energy edge states in bilayer graphene. Physical Review B, v. 81, n. 4, p. 045430, Jan. 2010.

[77] MONKHORST, H. J.; PACK, J. D. Special points for Brillouin-zone integrations. Physical Review B, v. 13, n. 12, p. 5188-5192, June 1976.

[78] MüLLER-PLATHE, F. A simple nonequilibrium molecular dynamics method for calculating the thermal conductivity. The Journal of Chemical Physics, v. 106, n. 14, p. 6082-6085, 1997.

[79] PLIMPTON, S. Fast Parallel Algorithms for Short-Range Molecular Dynamics. Journal of Computational Physics, v. 117, n. 1, p. 1-19, Mar. 1995.

[80] STUART, S. J.; TUTEIN, A. B.; HARRISON, J. A. A reactive potential for hydrocarbons with intermolecular interactions. The Journal of Chemical Physics, v. 112, n. 14, p. 6472, 2000.

[81] Berendsen, H. J. C.; POSTMA, J. P. M.; VAN GUNSTEREN, W. F.; DINOLA, A.; HAAK, J. R. Molecular dynamics with coupling to an external bath. The Journal of Chemical Physics, v. 81, n. 8, p. 3684, 1984.

[82] XU, X.; GABOR, N. M.; ALDEN, J. S.; VAN DER ZANDE, A. M.; MCEUEN, P. L. Photo-thermoelectric effect at a graphene interface junction. Nano letters, v. 10, n. 2, p. 562-6, Feb. 2010.

[83] BASKO, D. Applied physics. A photothermoelectric effect in graphene. Science, Washington, v. 334, n. 6056, p. 610-1, Nov. 2011. 
[84] GABOR, N. M.; SONG, J. C. W.; MA, Q.; NAIR, N. L.; TAYCHATANAPAT, T.; WATANABE, K.; TANIGUCHI, T.; LEVITOV, L. S.; JARILLOHERRERO, P. Hot carrier-assisted intrinsic photoresponse in graphene. Science, Washington, v. 334, n. 6056, p. 648-52, Nov. 2011.

[85] BOUKAI, A. I.; BUNIMOVICH, Y.; TAHIR-KHELI, J.; YU, J.-K.; GODDARD, W. A.; HEATH, J. R. Silicon nanowires as efficient thermoelectric materials. Nature, London, v. 451, n. 7175, p. 168-71, Jan. 2008.

[86] HOCHBAUM, A. I.; CHEN, R.; DELGADO, R. D.; LIANG, W.; GARNETT, E. C.; NAJARIAN, M.; MAJUMDAR, A.; YANG, P. Enhanced thermoelectric performance of rough silicon nanowires. Nature, London, v. 451, n. 7175, p. 163-7, Jan. 2008.

[87] BAUER, G. E. W.; SAITOH, E.; VAN WEES, B. J. Spin caloritronics. Nature materials, v. 11, n. 5, p. 391-9, May 2012.

[88] KARPAN, V.; GIOVAnNETTI, G.; KHOMYAKOV, P.; TALANANA, M.; STARIKOV, A.; ZWIERZYCKI, M.; VAN DEN BRINK, J.; BROCKS, G.; KELLY, P. Graphite and Graphene as Perfect Spin Filters. Physical Review Letters, Woodbury, v. 99, n. 17, p. 176602, Oct. 2007.

[89] TOMBros, N.; JOZSA, C.; POPINCIUC, M.; JONKMAN, H. T.; VAN WEES, B. J. Electronic spin transport and spin precession in single graphene layers at room temperature. Nature, London, v. 448, n. 7153, p. 571-4, Aug. 2007.

[90] DRAGOMAN, D.; DRAGOMAN, M. Giant thermoelectric effect in graphene. Applied Physics Letters, Woodbury, v. 91, n. 20, p. 203116, 2007. 
[91] ZHOU, B.; ZHOU, B.; LIU, Z.; ZHOU, G. Thermoelectric effect in a graphene sheet connected to ferromagnetic leads. Journal of Applied Physics, Woodbury, v. 112, n. 7 , p. $073712,2012$.

[92] WU, X.; HU, Y.; RUAN, M.; MADIOMANANA, N. K.; BERGER, C.; DE HEER, W. A. Thermoelectric effect in high mobility single layer epitaxial graphene. Applied Physics Letters, Woodbury, v. 99, n. 13, p. 133102, 2011.

[93] ZENG, M.; FENG, Y.; LIANG, G. Thermally induced currents in graphenebased heterostructure. Applied Physics Letters, Woodbury, v. 99, n. 12, p. $123114,2011$.

[94] NI, Y.; YAO, K.; FU, H.; GAO, G.; ZHU, S.; WANG, S. Spin seebeck effect and thermal colossal magnetoresistance in graphene nanoribbon heterojunction. Scientific reports, v. 3, n. 1380, p. 1380, Jan. 2013.

[95] ZENG, M.; HUANG, W.; LIANG, G. Spin-dependent thermoelectric effects in graphene-based spin valves. Nanoscale, v. 5, n. 1, p. 200-8, Jan. 2013.

[96] SCHWIERZ, F. Graphene transistors. Nature nanotechnology, v. 5, n. 7, p. 487-96, July 2010.

[97] CRETU, O.; KRASHENINNIKOV, A. V.; RODRÍGUEZ-MANZO, J. A.; SUN, L.; NIEMINEN, R. M.; BANHART, F. Migration and Localization of Metal Atoms on Strained Graphene. Physical Review Letters, Woodbury, v. 105, n. 19, p. 196102, Nov. 2010.

[98] SANTOS, E. J. G.; AYUELA, A.; SáNCHEZ-PORTAL, D. First-principles study of substitutional metal impurities in graphene: structural, electronic and magnetic properties. New Journal of Physics, v. 12, n. 5, p. 053012, May 2010. 
[99] CAO, C.; WU, M.; JIANG, J.; CHENG, H.-P. Transition metal adatom and dimer adsorbed on graphene: Induced magnetization and electronic structures. Physical Review B, v. 81, n. 20, p. 205424, May 2010.

[100] SANTOS, E. J. G.; SáNCHEZ-PORTAL, D.; AYUELA, A. Magnetism of substitutional Co impurities in graphene: Realization of single $\pi$ vacancies. Physical Review B, v. 81, n. 12, p. 125433, Mar. 2010.

[101] KRASHENINNIKOV, A.; LEHTINEN, P.; FOSTER, A.; PYYKKö, P.; NIEMINEN, R. Embedding Transition-Metal Atoms in Graphene: Structure, Bonding, and Magnetism. Physical Review Letters, Woodbury, v. 102, n. 12, p. 126807, Mar. 2009.

[102] TANG, Y.; YANG, Z.; DAI, X. Trapping of metal atoms in the defects on graphene. The Journal of chemical physics, v. 135, n. 22, p. 224704, Dec. 2011.

[103] BOUKHVALOV, D. W.; KATSNELSON, M. I. Destruction of graphene by metal adatoms. Applied Physics Letters, Woodbury, v. 95, n. 2, p. 023109, 2009.

[104] WANG, H.; WANG, Q.; CHENG, Y.; LI, K.; YAO, Y.; ZHANG, Q.; DONG, C.; WANG, P.; SCHWINGENSCHLöGL, U.; YANG, W.; ZHANG, X. X. Doping monolayer graphene with single atom substitutions. Nano letters, v. 12, n. 1, p. 141-4, Jan. 2012.

[105] RODRIGUEZ-MANZO, J. A.; BANHART, F. Creation of individual vacancies in carbon nanotubes by using an electron beam of 1 å diameter. Nano Letters, v. 9, n. 6, p. 2285-2289, 2009. 
[106] PERDEW, J. P.; BURKE, K.; ERNZERHOF, M. Generalized Gradient Approximation Made Simple. Physical Review Letters, Woodbury, v. 77, n. 18, p. 3865-3868, Oct. 1996.

[107] BüTTIKER, M.; IMRY, Y.; LANDAUER, R.; PINHAS, S. Generalized manychannel conductance formula with application to small rings. Physical Review $B$, v. 31, n. 10, p. 6207-6215, May 1985.

[108] KRASHENINNIKOV, A. V.; BANHART, F. Engineering of nanostructured carbon materials with electron or ion beams. Nature materials, v. 6, n. 10, p. 723-33, Oct. 2007.

[109] ROBERTSOn, A. W.; MONTANARI, B.; HE, K.; KIM, J.; ALLEN, C. S.; WU, Y. A.; OLIVIER, J.; NEETHLING, J.; HARRISON, N.; KIRKLAND, A. I.; WARNER, J. H. Dynamics of single Fe atoms in graphene vacancies. Nano letters, v. 13, n. 4, p. 1468-75, Apr. 2013.

[110] ROBERTSOn, A. W.; ALLEN, C. S.; WU, Y. A.; HE, K.; OLIVIER, J.; NEETHLING, J.; KIRKLAND, A. I.; WARNER, J. H. Spatial control of defect creation in graphene at the nanoscale. Nature communications, v. 3, p. 1144, Jan. 2012.

[111] ROCHA, A. R.; MARTINS, T. B.; FAZZIO, A.; DA SILVA, A. J. R. Disorderbased graphene spintronics. Nanotechnology, v. 21, n. 34, p. 345202, Aug. 2010.

[112] DE AlmeidA, J. M.; ROCHA, A. R.; DA SILVA, A. J. R.; FAZZIO, A. Spin filtering and disorder-induced magnetoresistance in carbon nanotubes: Ab initio calculations. Physical Review B, v. 84, n. 8, p. 085412, Aug. 2011.

[113] RATNER, M. A brief history of molecular electronics. Nature nanotechnology, v. 8, n. 6, p. 378-81, June 2013. 
[114] BRUOT, C.; HIHATH, J.; TAO, N. Mechanically controlled molecular orbital alignment in single molecule junctions. Nature nanotechnology, v. 7, n. 1, p. 35-40, Jan. 2012.

[115] BAHETI, K.; MALEN, J. A.; DOAK, P.; REDDY, P.; JANG, S.-Y.; TILLEY, T. D.; MAJUMDAR, A.; SEGALMAN, R. A. Probing the chemistry of molecular heterojunctions using thermoelectricity. Nano letters, v. 8, n. 2, p. 715-9, Feb. 2008.

[116] MALEN, J. A.; DOAK, P.; BAHETI, K.; TILLEY, T. D.; SEGALMAN, R. A.; MAJUMDAR, A. Identifying the length dependence of orbital alignment and contact coupling in molecular heterojunctions. Nano letters, v. 9, n. 3, p. 1164-9, Mar. 2009.

[117] KE, S.-H.; YANG, W.; CURTAROLO, S.; BARANGER, H. U. Thermopower of molecular junctions: an ab initio study. Nano letters, v. 9, n. 3, p. 1011-4, Mar. 2009.

[118] QUEK, S. Y.; CHOI, H. J.; LOUIE, S. G.; NEATON, J. B. Thermopower of amine-gold-linked aromatic molecular junctions from first principles. ACS nano, v. 5, n. 1, p. 551-7, Jan. 2011.

[119] WIDAWSKY, J. R.; DARANCET, P.; NEATON, J. B.; VENKATARAMAN, L. Simultaneous determination of conductance and thermopower of single molecule junctions. Nano letters, v. 12, n. 1, p. 354-8, Jan. 2012.

[120] WIDAWSKY, J. R.; CHEN, W.; VáZQUEZ, H.; KIM, T.; BRESLOW, R.; HYBERTSEN, M. S.; VENKATARAMAN, L. Length-dependent thermopower of highly conducting $\mathrm{Au}-\mathrm{C}$ bonded single molecule junctions. Nano letters, v. 13, n. 6, p. 2889-94, June 2013. 
[121] FILIPPETTI, A.; SPALDIN, N. Self-interaction-corrected pseudopotential scheme for magnetic and strongly-correlated systems. Physical Review B, v. 67, n. 12, p. 125109, Mar. 2003.

[122] PEMmARAJU, C.; ARCHER, T.; SáNCHEZ-PORTAL, D.; SANVITO, S. Atomic-orbital-based approximate self-interaction correction scheme for molecules and solids. Physical Review B, v. 75, n. 4, p. 045101, Jan. 2007.

[123] TOHER, C.; SANVITO, S. Efficient Atomic Self-Interaction Correction Scheme for Nonequilibrium Quantum Transport. Physical Review Letters, Woodbury, v. 99, n. 5, p. 056801, July 2007.

[124] TOHER, C.; SANVITO, S. Effects of self-interaction corrections on the transport properties of phenyl-based molecular junctions. Physical Review B, v. 77, n. 15, p. 155402, Apr. 2008.

[125] SONG, H.; KIM, Y.; JANG, Y. H.; JEONG, H.; REED, M. A.; LEE, T. Observation of molecular orbital gating. Nature, London, v. 462, n. 7276, p. 1039-43, Dec. 2009.

[126] JUNIOR, A. T. R. Defeitos em Nanofitas de Grafeno zigzag. 2008. Dissertação (Mestrado em Física) - Instituto de Física da Universidade de São Paulo, 2008.

[127] ROCHA, A.; ROSSI, M.; FAZZIO, A.; DA SILVA, A. Designing Real Nanotube-Based Gas Sensors. Physical Review Letters, Woodbury, v. 100, n. 17, p. 176803, Apr. 2008.

[128] SZABO, A.; OSTUlnd, N. S. Modern Quantum Chemistry. Dover, 1996.

[129] THOMAS, L. H. The calculation of atomic fields. Mathematical Proceedings of the Cambridge Philosophical Society, v. 23, n. 05, p. 542, Oct. 2008.

[130] FERMI, E. Un Metodo Statistico per la Determinazione di alcune Priorieta dell'Atome. Rend. Accad. Naz. Lincei, v. 6, p. 602-607, 1927. 
[131] HOHENBERG, P. Inhomogeneous Electron Gas. Physical Review, New York, v. 136, n. 3B, p. B864-B871, Nov. 1964.

[132] KOHN, W.; SHAM, L. J. Self-Consistent Equations Including Exchange and Correlation Effects. Physical Review, New York, v. 140, n. 4A, p. A1133A1138, Nov. 1965.

[133] CEPERLEY, D. M. Ground State of the Electron Gas by a Stochastic Method. Physical Review Letters, New York, v. 45, n. 7, p. 566-569, Aug. 1980.

[134] CAPELLE, K. A bird's-eye view of density-functional theory, 2002.

[135] PERDEW, J. P.; WANG, Y. Accurate and simple analytic representation of the electron-gas correlation energy. Physical Review B, v. 45, n. 23, p. 13244-13249, June 1992.

[136] BECKE, A. D. Density-functional exchange-energy approximation with correct asymptotic behavior. Physical Review A, Woodbury, v. 38, n. 6, p. 3098-3100, Sept. 1988.

[137] LEE, C.; YANG, W.; PARR, R. G. Development of the Colle-Salvetti correlation-energy formula into a functional of the electron density. Physical Review B, v. 37, n. 2, p. 785-789, Jan. 1988.

[138] CUDA - Compute Unified Device Architecture. Disponível em: https://developer.nvidia.com/cuda-toolkit.

[139] BLAS - Basic Linear Algebra Subprograms. Disponível em: http://www.netlib.org/blas/.

[140] LAPACK - Linear Algebra Package. Disponível em: http://www.netlib.org/lapack/. 
[141] CUBLAS - CUDA Basic Linear Algebra Subroutines. Disponível em: https://developer.nvidia.com/cuBLAS.

[142] MAGMA - Matrix Algebra on GPU and Multicore Architectures. Disponível em: http://icl.utk.edu/magma/.

[143] SANCHO, M. P. L.; SANCHO, J. M. L.; SANCHO, J. M. L.; RUBIO, J. Highly convergent schemes for the calculation of bulk and surface Green functions. Journal of Physics F: Metal Physics, v. 15, n. 4, p. 851-858, Apr. 1985.

[144] BRANDBYGE, M.; MOZOS, J.-L.; ORDEJóN, P.; TAYLOR, J.; STOKBRO, K. Density-functional method for nonequilibrium electron transport. Physical Review B, v. 65, n. 16, p. 165401, Mar. 2002. 